\title{
Theory of Mind in der Rezeption literarischer Erzähltexte
}

\section{Dissertation}

zur Erlangung des philosophischen Doktorgrades

an der philosophischen Fakultät

der Georg-August-Universität Göttingen

vorgelegt von

Stefanie Luther

geb. in Berlin

Göttingen 2017 



\section{Inhaltsverzeichnis}

I Einleitung ........................................................................................................... 1

1 Gegenstand der Arbeit................................................................................... 1

2 Motivierung der Fragestellungen und Zielsetzung …………............................... 3

3 Schnittstellen und Probleme im Rahmen interdisziplinärer theoretischer

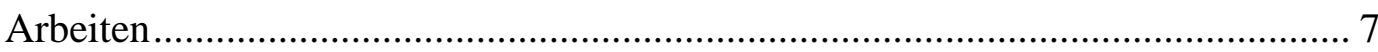

4 Aufbau und Vorgehensweise in dieser Arbeit .................................................... 15

II Was ist ,Theory of Mind'? ..................................................................................... 23

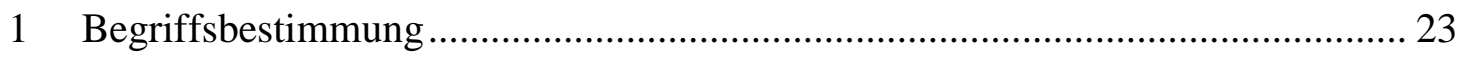

2 Die unterschiedlichen Funktionen der ToM im Kontext sozialer Interaktion ...... 27

2.1 Einführung: Die Interpretation psychologisch motivierter

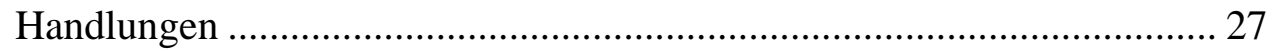

2.2 Einzelne Dimensionen der Handlungsmotivierung................................... 29

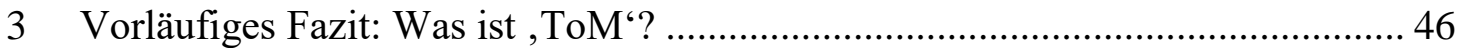

4 Wie funktioniert die ToM? - Grundlegende kognitive Mechanismen ................. 47

4.1 Die kognitive Repräsentation mentaler Haltungen …………………….... 47

4.2 Die Rolle der kognitiven ,Exekutiven Funktionen` für die ToM............... 56

$4.3 \quad$ ToM und Sprache ............................................................................... 58

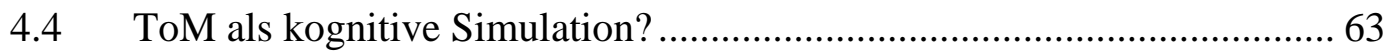

4.5 Narrative Strukturen des psychologischen Handlungsverständnisses ...... 68

5 Fazit - Was ist ToM und wie funktioniert sie? .................................................. 71

III Die Rolle der ToM in der literarischen Rezeption ................................................... 75

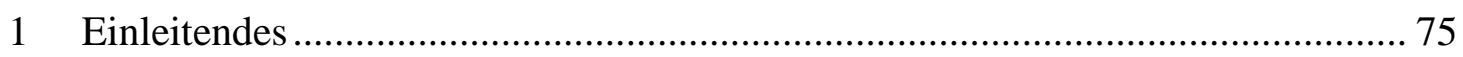

2 Initiation eines geeigneten Rezeptionsmodus .................................................. 78

3 Narrativität: Die Verarbeitung von narrativ vermittelten Informationen............... 84

3.1 Mentale Modelle als Basis von Verstehens- und

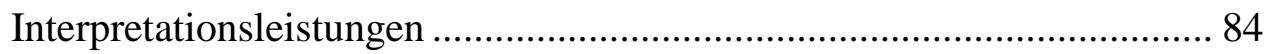

3.2 Die kognitive Konstruktion von Wahrnehmung / die perspektivgebundene Rekonstruktion fiktiver Ereignisse. 
3.3 Die grundlegende Rekursivität narrativ vermittelter Informationen ......... 98

4 Die metakognitive Verarbeitung literarischer Informationen ... 101

5 Fiktionsrezeption und die kognitive Repräsentation imaginativer Gegenstände

5.1 Die Imaginationsleistung und der eigene Standpunkt gegenüber der

Fiktionswelt ................................................................................ 107

5.2 Imagination als kognitive Grundhaltung.......................................... 111

5.3 Eigene Erlebnisse und Empfindungen, denen ein Imaginationsakt voransteht ..................................................................................... 113

6 Die Methode der kognitivistischen Textanalyse ........................................... 118

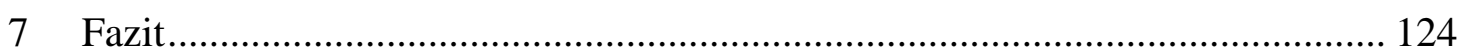

IV Die Parameter der kognitiven Rezeption im Fall einzelner Werke ...................... 131

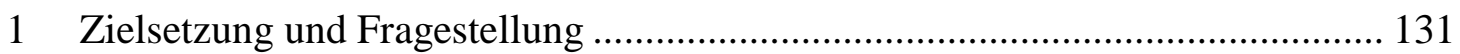

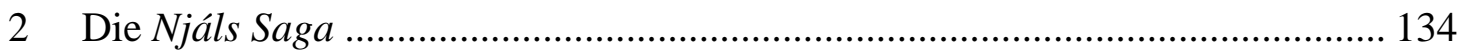

2.1 Einführung I: Die Handlung der Njáls Saga ........................................... 134

2.2 Einführung II: Kontext und wichtige Merkmale der Njáls Saga ........... 137

2.3 Die textgelenkte Rezeptionshaltung des Lesers .................................. 142

2.4 Besondere kognitive Leistungsanforderungen in der Njáls Saga ........... 164

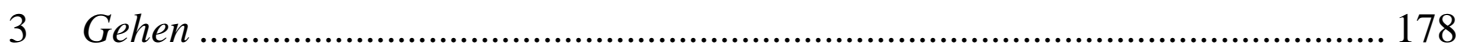

3.1 Einführung - Inhalt und bestehende Forschungsschwerpunkte ............. 178

3.2 Form: die Erzählsituation in Gehen .................................................. 182

3.3 Kognitive Rekonstruktion der fiktiven Handlung ............................... 186

3.4 Literarizitätsmerkmale und Metarepräsentation................................... 191

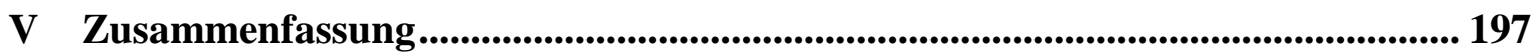

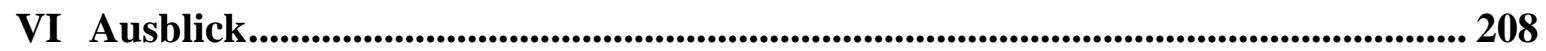

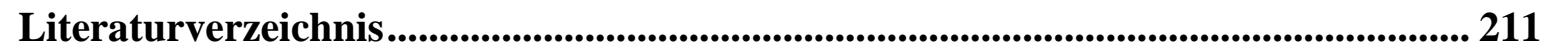

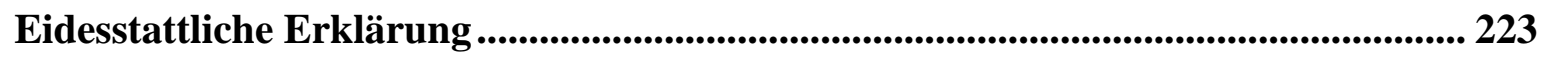




\section{Einleitung}

\section{Gegenstand der Arbeit}

Was geschieht, wenn wir ein literarisches Werk zur Hand nehmen und zu lesen beginnen? Häufig entsteht der Eindruck, dass sich die sprachlichen Zeichen des Textes (die Buchstaben auf dem Papier) unserer Wahrnehmung entziehen und vor unserem ,geistigen Auge“ eine neue Welt entsteht. Wie ist das zu erklären? Um diese Frage beantworten zu können, ist es notwendig, sich näher mit der menschlichen Kognition zu beschäftigen. Mit dem Ziel, den Zusammenhang zwischen der Kognition des Menschen und den spezifischen Merkmalen literarischer Texte genauer zu untersuchen, begannen Literaturwissenschaftler bereits in den 1980er Jahren ihre Thesen mit empirisch fundierten Erkenntnissen aus den Forschungsdisziplinen der Kognitions-, Entwicklungs- und Sozialpsychologie, der Neurologie und der kognitiven Linguistik zu stützen. ${ }^{1}$ Dieser Ansatz ist auch für die vorliegende Arbeit richtungsweisend. Dabei steht ein besonderer Teil der menschlichen Kognition - die Theory of Mind - im Zentrum des Interesses. Das Ziel dieser Arbeit ist es, die Rolle der Theory of Mind (nachfolgend ToM) im Rahmen der kognitiven Rezeption literarischer Erzähltexte genauer zu erfassen, als es im Rahmen vorangehender literaturwissenschaftlicher Arbeiten geschehen ist. Es gilt die These zu stärken, dass die ToM nicht nur im Zuge des Verstehens literarisch dargestellter sozialer Handlungen zum Einsatz kommt, sondern eine wesentlich zentralere Rolle für den gesamten Rezeptionsprozess übernimmt.

Der Begriff ,Theory of Mind‘, der von den Psychologen David Premack und Guy Woodruff im Jahr 1978 geprägt wurde, bezeichnet die Fähigkeit eines Individuums, sich selbst und anderen mentale Haltungen oder Vorgänge - z. B. Gedanken, Überzeugungen, Wünsche, Intentionen u. Ä. - zuzuschreiben (Premack \& Woodruff, 1978). Die Attribuierung innerer

\footnotetext{
${ }^{1}$ Einen neueren Überblick über dieses Forschungsfeld und die zahlreichen untersuchten Teilprobleme der kognitiven literarischen Rezeption bietet die Arbeit von Sophia Wege (2013).
} 
Vorgänge ist für die menschliche Interaktion essenziell. Das ist zum Beispiel dann sichtbar, wenn eine Diskrepanz zwischen der Realität und der mentalen Repräsentation dieser Realität besteht - eine Person etwa, die auf Grund einer falschen Überzeugung oder unzureichenden Wissens handelt. Wenn wir jemanden dabei beobachten, wie er trotz strahlenden Sonnenscheins zum Regenschirm greift, können wir dank unserer ToM auf eine Grundausstattung kognitiver Konzepte zurückgreifen, die uns dabei helfen, die ,mentale Welt' dieser Person zu erschließen und der beobachteten Handlung eine entsprechende Handlungsmotivierung zuzuordnen. Menschen sind dazu in der Lage, Handlungsmotivierungen auf zum Teil nicht direkt sichtbare, innere‘ Faktoren zurückzuführen und dabei einzukalkulieren, dass diese potenziell nicht mit den Tatsachen oder dem eigenen Wissensstand übereinstimmen. So können wir Annahmen darüber bilden, welche mentalen Haltungen die oben genannte Person dazu veranlasst haben könnten, zum Regenschirm zu greifen - denkbar wäre z. B. die Überzeugung, dass es im Laufe des Tages regnen wird, oder aber die fälschliche Überzeugung, es regne tatsächlich.

Wenn es darum geht, die Bedeutung der ToM zu veranschaulichen, werden sowohl in literatur- als auch kognitionswissenschaftlichen Arbeiten gern - wie auch hier - Beispiele konstruiert, in denen es darum geht, dass Menschen mit Hilfe der Attribuierung mentaler Haltungen und Vorgänge die Handlungsmotivierungen anderer bestimmen. Damit ist die Funktion der ToM für die menschliche soziale Interaktion und Kognition jedoch nicht vollständig erfasst. Es ist schwierig, eine umfassende Definition dieses Konzeptes zu finden, da sich die einzelnen kognitionswissenschaftlichen Arbeiten häufig lediglich mit einzelnen Teilfunktionen der ToM befassen. Eine umfassende Vorstellung des ToM-Konzeptes ist daher Aufgabe des ersten Arbeitskapitels. Daran anschließend lautet die Frage: Welche Rolle spielt die ToM im Rahmen der Rezeption literarischer Texte? Diese grundsätzliche Fragestellung lässt sich in folgende einzelne Teilfragen aufgliedern:

1. In welcher Form sind die kognitiven Mechanismen der ToM an der kognitiven Rezeption literarischer Erzähltexte beteiligt?

2. Besteht ein besonderer Zusammenhang zwischen den Spezifika literarischer Texte und der ToM-relevanten Kognitionsleistung des Lesers?

3. Lassen sich mit Hilfe der gründlichen Kenntnis der kognitiven Mechanismen der ToM und an Hand systematischer Textanalysen fundierte Annahmen über die erforderlichen Kognitionsleistungen des Lesers während der Lektüre eines bestimmten Textes formulieren? 
4. Welche Erkenntnisse lassen sich mit Hilfe dieser, unter 3. genannten Vorgehensweise über die Gemeinsamkeiten und Unterschiede in der kognitiven Rezeption zweier grundverschiedener literarischer Erzähltexte gewinnen?

\section{Motivierung der Fragestellungen und Zielsetzung}

Obwohl die oben formulierten Fragen in der Vergangenheit zum Teil in ähnlicher Form bereits gestellt wurden, steht eine genaue und umfassende Studie der mit der ToM verbundenen kognitiven Vorgänge im Zusammenhang mit den verschiedenen (Re-)Konstruktionsleistungen und Sinngebungsprozessen während der Lektüre eines literarischen Textes bis heute aus. Lisa Zunshines Monographie Why we read fiction. Theory of Mind and the novel aus dem Jahr 2006 stellt als erste literaturwissenschaftliche Arbeit mit explizitem Bezug zum Begriffsrepertoire der ToM-Forschung und mit Referenzen zu den Einsichten der kognitiven Entwicklungspsychologie eine Pilotstudie auf diesem Gebiet dar. Die Autorin vertritt dort die These, dass die Interpretation von literarisch dargestellten Handlungen ebenso wie das Verstehen von Handlungen im realen sozialen Kontext auf den gleichen, mit der ToM verbundenen Kognitionsprozessen beruht. Dem Analogieprinzip entsprechend basiert diese Argumentation auf der Annahme, dass die Wahrnehmung und das Verstehen erfundener Handlungen (bspw. aus dem Kontext eines fiktionalen Erzähltextes) und die Wahrnehmung realer Personen, Ereignisse und Handlungen mit Hilfe derselben kognitiven Mechanismen erfolgen. ${ }^{2}$ Eine Begründung, die diese Annahme plausibel macht, findet Zunshine in denjenigen kognitionswissenschaftlichen Arbeiten, die sich mit dem Problem des Autismus-Spektrums, einer frühkindlichen Entwicklungsstörung, beschäftigen. ${ }^{3}$ Kinder, die an dieser Entwicklungsstörung leiden, verfügen nicht (oder nur in eingeschränktem Maße) über die Fähigkeit, das Verhalten anderer auf die mentalen Haltungen und inneren Vorgänge der handelnden Personen zurückzuführen. Gleichzeitig haben Menschen mit einer stärker oder schwächer ausgeprägten Störung des Autismus-Spektrums häufig Schwierigkeiten, literarische Erzähltexte zu verstehen bzw. zeigen nur wenig Interesse am Lesen solcher Texte. Diese Beobachtungen führen Zunshine zu der Hypothese, dass die ToM, die im Fall realer sozialer Interak-

\footnotetext{
${ }^{2}$ Zum Analogieprinzip vgl. Wege (2013, S. 51).

${ }^{3}$ Zunshine bezieht sich dabei hauptsächlich auf Arbeiten von Simon Baron-Cohen (z. B. 1997), die in diesem Forschungsfeld richtungsweisend sind.
} 
tionen zum Einsatz kommt, auch dafür genutzt werden muss (bzw. im Fall autistischer Patienten nicht dafür genutzt werden kann), fiktive Charaktere im Zuge der kognitiven Textinterpretation ,mental' mit psychologischen Eigenschaften auszustatten. ${ }^{4}$

Wenn eine unzureichend entwickelte ToM tatsächlich mit Verständnisschwierigkeiten während der Lektüre literarischer Texte zusammenhängt, dann scheint die Annahme bestätigt, dass ein Leser seine ToM im Zuge der literarischen Rezeption nutzt. Daran anschließend stellt sich die Frage, in welcher Form die ToM im Zuge der literarischen Rezeption zum Einsatz kommt. Den Hypothesen, die Zunshine diesbezüglich aufstellt, liegt allerdings nur eine sehr knappe Definition des ToM-Begriffs zu Grunde, der sich weitestgehend an die geläufige allgemeine Bestimmung des Terminus anlehnt. Die ToM wird hier auf einer allgemeinen Ebene als eine menschliche Fähigkeit verstanden, die es uns gestattet, sich selbst und anderen mentale Haltungen zuzuschreiben. Die Rolle der menschlichen ToM wird in Zunshines Arbeit - möglicherweise daher - ausschließlich in Bezug auf die Handlungsebene und Diegese, d. h. in Bezug auf die Ausstattung literarischer Figuren und anderer anthropomorpher Einheiten mit mentalen Haltungen und Vorgängen, untersucht. Demnach ist ein Leser in der Lage, (a) fiktiven Figuren (und ggf. dem Erzähler) aktiv mentale Haltungen zuzuschreiben, wenn dies zugunsten des Textverständnisses notwendig ist (vgl. Zunshine, 2006, S. 3) $)^{5}$ und (b) die Attribuierungsprozesse der fiktiven Figuren (und des Erzählers) untereinander zu identifizieren und diese Einsichten für das Textverständnis zu nutzen. Beispielhaft sei hier ein Ausschnitt aus Zunshines Interpretation von S. Richardsons Clarissa (1784) zitiert. Sie schreibt: „Here is what happens. Clarissa's parents, siblings, and uncles are angry at her because she refuses to marry an obnoxious wealthy suitor of their providing. Her rejection of that man, they are convinced, stems from her secret preference for Lovelace.“ (Vgl. Zunshine, 2006, S. 86). Zunshine weist in diesem Zusammenhang auf das Potenzial literarischer Erzähltexte hin, die entsprechenden Kognitionsprozesse des Lesers durch komplexe fiktive Szenarien besonders intensiv anzusprechen oder gar zu überfordern. Für den Autor eines literarischen Textes besteht also die Möglichkeit, durch besondere Justierungen auf der Handlungs- und Erzählebene den Anspruch an die Kognitionsleistung des Lesers im Vergleich mit üblichen realen sozialen Situationen zu variieren und zu (über-)steigern (vgl. Zunshine, 2010, S. 206). Beispiele solcher narrativer Strategien, die Zunshine ins

\footnotetext{
${ }^{4}$ Die wichtigsten Thesen Zunshines und weitere relevante Forschungsarbeiten, die sich mit der Rolle der ToM im Zuge der literarischen Rezeption beschäftigen, werden an geeigneter Stelle zu Beginn des zweiten Hauptteils dieser Arbeit in Form eines Forschungsüberblicks noch einmal ausführlich vorgestellt.

${ }^{5}$ Zunshines Interpretation einer exemplarisch ausgewählten Textstelle aus dem Roman Mrs. Dalloway von Virginia Woolf lautet: „Walsh's body language betrays his agitation, his joy, and his embarrassment and that the meeting has brought back the old days [...]" (Zunshine 2006, S. 3).
} 
Feld führt, sind z. B. die übermäßige Betonung oder ungewöhnlich spärliche Ausführung bestimmter psychologischer Vorgänge (overreporting und undertelling). Einige Autoren (wie etwa Henry James) lenken die Aufmerksamkeit ihrer Leser, durch einen expliziten Fokus auf innere Vorgänge, vermehrt auf die mentalen Vorgänge fiktiver Figuren und stimulieren die relevanten Kognitionsprozesse des Lesers auf diesem Weg in besonderem Maße (vgl. Zunshine, 2006, S. 24). Autoren wie Ernest Hemingway und Virginia Woolf hingegen weisen handlungsmotivierende innere Vorgänge an vielen Stellen nicht aus, wobei sie auf diese Weise eine eigenständige Attribuierungsleistung des Lesers einfordern (vgl. Zunshine, 2006, S. 23). Zunshine zeigt im Zuge ihrer Textanalysen, dass Woolf ihre Leser in dieser Hinsicht vor eine besondere Herausforderung stellt, da ihre Texte für eine gelingende Handlungsinterpretation die Entschlüsselung mehrfach verketteter mentaler Haltungen (X möchte, dass Y denkt, dass Z Bescheid weiß etc.) erforderlich macht (Zunshine, 2010, S. 194). Dies sei deshalb möglich, weil Woolf mit Hilfe ihrer eigenen ToM die Kognitionsleistungen ihrer Leser antizipieren und während der Textproduktion berücksichtigen kann (Zunshine, 2006, S. 22).

Zunshine gelingt es, den Zusammenhang zwischen der allgemeinen Funktion der ToM in der menschlichen sozialen Interaktion und ihrer Funktion für die Interpretation literarisch vermittelter Handlungen plausibel darzustellen. Auf Grund einer eher oberflächlichen Bestimmung des ToM-Begriffes gelingt es ihr jedoch nicht, die Rolle der ToM für die literarische Rezeption detaillierter vorzustellen. Um dies zu leisten, ist ein genauerer Blick auf die kognitionswissenschaftliche Forschung zu dieser besonderen menschlichen Fähigkeit notwendig. Dort werden heute eine Reihe unterschiedlicher Fragestellungen bearbeitet, die sich mit der phylogenetischen und ontogenetischen Entwicklung der ToM, mit ihren hirnphysiologischen Grundlagen und möglichen Zusammenhängen zwischen der ToM und weiteren kognitiven Fähigkeiten beschäftigen. Die zahlreichen Erkenntnisse, die im Rahmen dieser Forschungsarbeiten gesammelt wurden, ermöglichen detailliertere Einblicke in die kognitiven Mechanismen, die der ToM zugrunde liegen. Die vorliegende Arbeit soll einen Beitrag dazu leisten, die Rolle der ToM im Rahmen des literarischen Rezeptionsprozesses gründlicher zu untersuchen. Diese Rolle geht m. E. über die (analog zum realen sozialen Kontext geleistete) Attribuierung psychologischer Eigenschaften hinsichtlich der durch den Text evozierten (fiktiven) Instanzen hinaus. Ohne die kognitiven Mechanismen der ToM, so meine zentrale Annahme, wäre der Mensch nicht nur nicht in der Lage, die im Text darge- 
stellten sozialen Interaktionen zu verstehen. Auch weitere Elemente, die für unseren gewohnten Umgang mit literarischen Erzähltext zentral sind, stehen in elementarem Zusammenhang mit der ToM. Welche das sind wird im Laufe der Arbeit zu klären sein.

Ein erstes Ziel dieser Arbeit ist es, die Hypothese zu stärken, dass die ToM und die ihr zugrunde liegenden kognitiven Mechanismen in der literarischen Rezeption eine zentralere Rolle übernehmen als bisher angenommen. Es gilt herauszufinden, ob sich mit Hilfe einer besseren Kenntnis ihrer kognitiven Mechanismen und Ressourcen Funktionen der ToM im Zusammenhang mit der Lektüre literarischer Texte identifizieren lassen, die bisher noch nicht benannt wurden. Um dies zu erreichen, ist es notwendig, das Globalkonzept ,ToMFähigkeit‘ nach einzelnen beteiligten kognitiven Funktionen für die soziale Interaktion aufzuschlüsseln. Außerdem wird die Relevanz spezifisch literarischer Eigenschaften für die Verarbeitung mit Hilfe der kognitiven Funktionen des Menschen besonders zu berücksichtigen sein. Solche literarischen Texteigenschaften sollten sich mittels vorhandener literaturtheoretischer Konzepte bestimmen und mit bestimmten kognitiven Funktionen in einen $\mathrm{Zu}-$ sammenhang stellen lassen. Diese Vorgehensweise gestattet es, die vielfältigen Formen des Zusammenwirkens von Textmerkmalen und Leserkognition im Einzelnen unterscheiden und beschreiben zu können. Das zweite Ziel dieser Arbeit besteht dementsprechend darin, den Zusammenhang zwischen typischen Merkmalen literarischer Erzähltexte und den Mechanismen der ToM zu untersuchen. Zu diesem Zweck dienen bestehende rezeptionspsychologische und literaturtheoretische Ansätze als Hinweisquelle auf die Wirkungsmechanismen zwischen typischen literarischen Textmerkmalen und der Leserkognition. Ein drittes Ziel ist es, mittels der exemplarischen Analyse zweier unterschiedlicher literarischer Texte herauszufinden, welche ToM-relevanten Prozesse die Informationsstruktur eines bestimmten literarischen Textes aktiviert.

Die vorliegende Arbeit reiht sich mit diesen drei Arbeitszielen in die Reihe der Bemühungen ein, die bisher schwer zugängliche, vielschichtige Rolle der ToM in der Rezeption literarischer Texte genauer zu erfassen. Es gilt, die These zu plausibilisieren, dass die ToM eine zentrale Funktion in wichtigen Bereichen der literarischen Rezeption (und nicht nur im Zuge des Verstehens literarisch dargestellter Handlungen und Kommunikation) übernimmt. Da es sich dabei um ein exploratives Vorhaben handelt, besteht ein viertes Ziel darin, zu klären, bis zu welchem Grad sich die untersuchten Fragen mit den hier zur Verfügung stehenden methodischen Mitteln zufriedenstellend beantworten lassen. Eine Aufgabe, die in dieser Arbeit berücksichtigt werden soll ist es, einer Reihe von methodischen Problemen, die mit der 
Verbindung kognitionswissenschaftlicher und literaturtheoretischer Fragestellungen einhergehen, kritisch zu begegnen. Diese Probleme betreffen hauptsächlich die sinnvolle Verwendung von Konzepten und Annahmen der Kognitionspsychologie, um literaturwissenschaftliche Fragestellungen zu beantworten. Julia Mansour spricht diesbezüglich von der „Scylla uneingelöster Versprechungen und der Charybdis der Trivialität“" (Mansour, 2009, S. 156) ${ }^{6}$ und mahnt zu Recht zur methodischen Redlichkeit. Sie kritisiert u. a., dass Zunshine in ihren Arbeiten genauere methodologische Erläuterungen dazu, wie eine fruchtbare Verbindung zwischen den beiden Disziplinen der Kognitions- und Literaturwissenschaft schlüssig umgesetzt werden kann, schuldig bleibt. Ebenfalls offen ist, welche literaturwissenschaftlichen Fragestellungen mit Hilfe des ToM-Konzeptes stichhaltig beantwortet werden können und welche nicht (vgl. ebd., S. 158). Darüber hinaus gilt es zu klären, ob sich das Konzept der ToM dazu eignet, im Zuge einer entsprechend gestalteten konkreten Textanalyse sinnvolle Erkenntnisse zu gewinnen.

\section{Schnittstellen und Probleme im Rahmen interdisziplinärer theoretischer Arbeiten}

Zunshine gibt an einleitender Stelle in ihrer Arbeit an, dass ausreichend empirisch fundierte, kognitionswissenschaftliche Ergebnisse zur Verfügung stünden, um Antworten auf rezeptionspsychologische (,Is it possible that literary fiction builds on our capacity for mind-reading but also tries its limits?“) und literarhistorische (,How do different cultural-historical milieus encourage different literary explorations of this capacity?“) Fragen zu finden (vgl. Zunshine, 2006, S. 27). Dieser Feststellung stimme ich generell zu. Allerdings ist zu bedenken, dass die von Zunshine gewählte Methodik der Interpretation einzelner Textbeispiele aus unterschiedlichen kulturhistorischen Epochen lediglich zu begründeten Vermutungen und nicht zu stichhaltigen Ergebnissen hinsichtlich solcher Fragestellungen führen kann. Welche konkreten kognitiven Interpretationsvorgänge während der Rezeption einzelner literarischer Texte tatsächlich stattfinden, lässt sich im Zuge von solchen exemplarischen Interpretationen nur schwer erschließen. Das liegt v. a. an einem der Methodik inhärenten ,Beobachterproblem‘. Das bedeutet, dass eine objektive Erforschung kognitiver Vorgänge (z.

B. der Aktivität ToM-relevanter kognitiver Mechanismen während der Lektüre literarischer

\footnotetext{
${ }^{6}$ Hervorhebungen durch die Autorin.
} 
Texte) mit Hilfe des eigenen kognitiven Systems (u. a. der eigenen ToM-relevanten kognitiven Mechanismen) schwierig ist. Es ist wahrscheinlich, dass die Ergebnisse eines solchen Vorgehens durch einen ,false consensus effect ${ }^{\star}$ beeinflusst werden, wobei die Erwartungshaltung eines Beobachters bei der Interpretation von fremden Handlungen und mentalen Vorgängen durch eigene Denk- und Verhaltensstrukturen beeinflusst wird (vgl. Alicke \& Largo, 1995). In dieser Arbeit ist ein solcher Subjektivitätsbias dann problematisch, wenn es darum gehen soll, Vorhersagen über Kognitionsprozesse im Zusammenhang mit der Interpretation konkreter Textstellen zu treffen. Diese Vorhersagen beruhen notwendigerweise zu Teilen auf eigenen - in diesem Fall meinen - individuellen Schlussfolgerungen. Diese Schlussfolgerungen kommen dadurch zustande, dass ich Lesern bestimmte kognitive Interpretationsvorgänge attribuiere. Diese Attribution von Interpretationsvorgängen beruht auf einer detaillierten Analyse des Zusammenhanges zwischen den kognitiven Mechanismen der ToM und typischen oder individuellen Merkmalen literarischer Erzähltexte. Das oben genannte Beobachterproblem lässt sich minimieren, indem in der vorliegenden Arbeit - anders als in früheren Studien zu diesem Thema - die einzelnen kognitiven Funktionen der ToM detaillierter aufgliedert werden. Dabei beziehe ich sowohl Ergebnisse aus empirischen Versuchen als auch aus den theoretischen Arbeiten über die ToM sowie die der Forschung bekannten Prinzipien kognitiver Repräsentation und Modellbildung ein. Gleichzeitig dienen verschiedene literaturtheoretische Ansätze als Hinweisquelle dafür, welche kognitiven Prozesse für die literarische Rezeption besonders relevant sind. Das Interesse gilt genauer gesagt den online-Kognitionsprozessen, die der literarischen Rezeption unterliegen und einen Bezug zur ToM besitzen. Diese Prozesse, die in dieser Arbeit synonym auch als ,Rezeptions“und ,Interpretationsprozesse“ bezeichnet werden, betreffen den kognitiven Vorgang, der während der Lektüre eines literarischen Textes abläuft. Er ist von dem interpretativen Urteil über ein literarisches Werk zu unterscheiden, zu dem ein Leser aufgrund dieses Vorganges gelangt und das ggf. zusätzlich durch offline-Interpretationsprozesse getragen wird, die nicht während der Lektüre selbst, sondern zeitversetzt stattfinden. Zwischen online-Rezeptionsvorgängen im Allgemeinen und dem jeweils assoziierten Rezeptionsurteil besteht bis zu einem gewissen Grad ein Abhängigkeitsverhältnis. Ich gehe davon aus, dass die während der Textlektüre ablaufenden Interpretationsprozesse aufgrund der Textkonfiguration und aufgrund der dem Menschen gegebenen kognitiven Architektur zu bestimmten interpretativen Urteilen führen. Interpretationsurteile, aber auch der online-Rezeptionsprozess, werden durch eine Reihe von Interpretationsdispositionen des einzelnen Lesers beeinflusst. Dazu gehören z. B. seine Vorerfahrungen, sein individueller Wissensschatz über die Welt, seine 


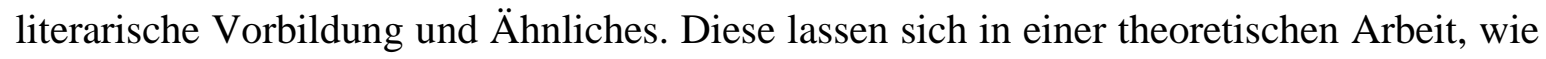
der vorliegenden, schwer einberechnen. Aus diesem Grund steht im Folgenden lediglich der online ablaufende Rezeptionsvorgang im Zentrum des Interesses, der in einem direkten Bezug zu den Merkmalen eines Textes steht. Es ist nicht möglich, an dieser Stelle den Dispositionen empirischer Leser Beachtung zu schenken. Daher ist es nicht sinnvoll, auch auf diese individuellen Voraussetzungen, die ein bestimmter empirischer Leser mit sich bringen könnte, Rücksicht zu nehmen. Deshalb wird hier auf das Konzept des Modell-Lesers zurück gegriffen. Wenn innerhalb der Argumentation dieser Arbeit von einem Leser oder Rezipienten die Rede ist, und nichts anderes explizit ausgewiesen wird, ist immer ein Modell-Leser gemeint. Ein solcher Modell-Leser soll grundsätzlich über alle notwendigen kognitiven Kompetenzen verfügen, um die von einem Text erforderten Operationen erfolgreich durchzuführen und auf diesem Weg zu einem kohärenten Textverständnis zu gelangen. Da er ein hypothetisches anthropomorphes Konstrukt ist, können ihm außerdem variable Eigenschaften zugesprochen werden, um bestimmte Fragestellungen zu untersuchen (vgl. Jannidis, 2004, S. 254).

Wie Alan Palmer gehe ich davon aus, dass es (a) grundsätzlich möglich ist, mit Hilfe der Kenntnis über bestimmte Prinzipien der menschlichen Kognition und über typische oder individuelle Eigenschaften literarischer Werke einen Modell-Leser zu konstruieren, der abgesehen von individuell unterschiedlichen kognitiven Dispositionen - einem empirischen Leser entspricht, und (b) die so gewonnenen Einsichten mit Einschränkungen daher auf den empirischen Leser übertragbar sind (vgl. Palmer, 2004, S. 18). Die bereits vorhandenen und z. T. empirisch gestützten Daten über die Architektur der menschlichen Kognition bilden eine geeignete Grundlage für die Konstruktion eines solchen Modell-Lesers. Ein solcher Modell-Leser wird hier so verstanden, dass er über die kognitiven Mechanismen eines gesunden Erwachsenen verfügt und einen literarischen Text ohne spezifisches historisches oder literarisches Vorwissen liest, um ihn zu verstehen. Unter der Voraussetzung, dass diese Parameter gleich bleiben, ist es möglich, 1. den Zusammenhang zwischen typischen Merkmalen literarischer Erzähltexte zu untersuchen und 2. die Wirkungsmechanismen zwischen der individuellen Textkonfiguration und den kognitiven Funktionen eines Lesers zu besprechen. Diese Vorgehensweise gestattet es sowohl Leser-zentrierte rezeptionspsychologische als auch Text-zentrierte Hypothesen zu diskutieren. Es soll vorliegend nicht darum gehen, die tatsächliche Wirkung eines Textes mit Bezug zu einem empirischen Leser im Einzelfall nachzuzeichnen, sondern das Wirkungspotenzial literarischer Texte abzustecken. Das Ziel ist es, einen systematischen Überblick über diejenigen Kognitionsprozesse zu erlangen, die 
in die Rezeption eines literarischen Erzähltextes eingebunden sind und Aussagen über die Relationen zwischen textuellen Merkmalen und kognitiven Prozessen zu treffen.

Auf diesem Weg soll der Erkenntnisraum der Literaturwissenschaft, der durch eine detaillierte Kenntnis der ToM gewonnen werden kann, ausgelotet werden. Es sei allerdings an dieser Stelle bereits angemerkt, dass bestehende literaturwissenschaftliche Theorien in dieser Arbeit nicht mit Hilfe des hier im Fokus stehenden kognitionswissenschaftlichen Konzeptes umgeschrieben werden sollen. Am Ende entsteht so keine neue ,ToM-Theorie der literarischen Interpretation', die ein eigenständiges Methodenwerkzeug für die Interpretation literarischer Texte anbietet. Allerdings ist es möglich, umfassendere Einblicke in die fundamentale Rolle, die die ToM während der Lektüre literarischer Texte übernimmt, zu gewinnen. Diese Einblicke sind deshalb wertvoll, weil sie es ermöglichen, zahlreiche unterschiedliche Fragestellungen in Zukunft präziser untersuchen zu können, die in den Arbeiten von Zunshine und in anderen literaturwissenschaftlichen Arbeiten, die sich im weiteren Sinne mit dem Zusammenhang zwischen der ToM und der literarischen Rezeption beschäftigen, bereits angesprochen wurden. Es ist an dieser Stelle sinnvoll, einen Überblick über die Gegenstandsbereiche dieser Fragestellungen zu erstellen und zu klären, welche Ansätze in der vorliegenden Arbeit verfolgt werden können und welche nicht.

\section{(1) Leser-zentrierte Hypothesen}

Diese Gruppe von Hypothese lässt sich auch als ,Voraussetzungs-Hypothesen“ umschreiben. Grundlage ist hier die Annahme, dass bestimmte kognitive Funktionen eine notwendige Voraussetzung für die Verarbeitung bestimmter Phänomene der literarischen Rezeption darstellen. Das bedeutet, dass ohne die jeweiligen (teilweise noch zu identifizierenden) kognitiven Funktionen der ToM der Umgang mit Literatur so, wie wir ihn gewohnt sind, nicht möglich wäre. In diesem Zusammenhang stehen hauptsächlich die ,top-down`- gerichteten kognitiven Prozesse während der Lektüre eines Textes im Fokus des Interesses. ,Top-down“ bedeutet, dass die Verarbeitung eines Stimulus von der Beschaffenheit bzw. den Funktionen der menschlichen Kognition bestimmt wird (Sarter, Givens \& Bruno, 2001, S. 147 f.). Ausschlaggebend für die Wahrnehmung oder Interpretation eines Signals - in diesem Fall einer Textpassage - sind hier die kognitiven Regeln und Wissenseinheiten, die der Leser mitbringt. Dem gegenüber steht das Prinzip der, bottom-up ${ }^{`}$-Verarbeitung von Umweltreizen. In diesem Fall wird die Verarbeitung der entsprechenden Informationen hauptsächlich von 
den Charakteristika des Stimulus selbst gelenkt. ${ }^{7}$ Lisa Zunshines Annahme, der Leser ,vervollständige“ die durch einen Text gegebenen Informationen mit Hilfe seiner ToM, um einen kohärenten Sinnzusammenhang herzustellen, ist ein passendes Beispiel für eine Leserzentrierte Hypothese.

Auch im Rahmen der vorliegenden Arbeit richtet sich die Aufmerksamkeit auf konkrete Kognitionsprozesse, die durch die Lektüre eines literarischen Erzähltextes erzeugt und gelenkt werden. Der Fokus liegt im Besonderen auf einer spezifischen Gruppe kognitiver Mechanismen, die mit dem kognitionspsychologischen Konzept der ToM zusammengefasst sind. Die zentrale Annahme, die es in dieser Arbeit zu prüfen gilt, lautet, dass die ToM eine entscheidendere Rolle im Rahmen der Rezeption literarischer Erzähltexte übernimmt, als bisher angenommen. Wie diese Rolle im Einzelnen zu definieren ist, soll auf Basis einer detaillierten Aufschlüsselung des ToM-Konzeptes untersucht werden. Die Frage, die dabei im Zentrum des Interesses steht lautet: Welche ToM-relevanten Kognitionsprozesse werden während der Lektüre eines typischen literarischen Erzähltextes angesprochen bzw. gebraucht?

(2) Text-zentrierte Hypothesen zur Relation zwischen Textmerkmal und Leserkognition

Im Rahmen Text-zentrierter Untersuchungen richtet sich die Aufmerksamkeit auf die ,bottom-up'-orientierte Verarbeitung von Informationen, wobei die spezifischen Charakteristika des literarischen Textes von entscheidender Bedeutung sind. Dabei geht es überwiegend darum, diejenigen Merkmale zu identifizieren, die Einfluss auf die kognitiven Prozesse nehmen, die während der Lektüre ablaufen. Der Fokus richtet sich entsprechend auf typische oder individuelle Merkmale literarischer Texte. Diese werden daraufhin untersucht, ob sich ein Zusammenhang mit der Leserkognition erfassen lässt oder nicht. Um eine Fragestellung dieser Art beantworten zu können, ist es notwendig, die spezifischen Merkmale literarischer Texte genau zu erfassen. Hierbei helfen die aus bereits geleisteten literaturtheoretischen Arbeiten gewonnenen Überlegungen ebenso wie das begriffliche Instrumentarium der Narratologie. Eine Unterkategorie dieses Gegenstandsbereiches bilden werkspezifische kognitivistische Hypothesen.

\footnotetext{
${ }^{7}$ Der Konsens der Neuro- und Kognitionswissenschaften lautet, dass es sich bei ,top-down'- und ,bottomup'- Prozessen um Verarbeitungsprinzipien handelt, die einander überlappen und im konkreten Fall zum Teil schwer voneinander zu trennen sind (Sarter, Givens \& Bruno, 2001, S. 148).
} 
(2a) Werkspezifische kognitivistische Hypothesen

Hier gilt die grundsätzliche Annahme, dass sich ein bestimmter literarischer Text als ,Anleitung‘ zur kognitiven Rezeption lesen lässt. Ist dies der Fall, dann wäre es möglich, an Hand einer systematischen Analyse von Struktur und Semantik eines einzelnen Werkes, Aussagen über die kognitiven Anforderungen der Lektüre dieses Werkes zu treffen. So können diejenigen Strukturen einzelner Werke identifiziert werden, die Leser vor ganz bestimmte, beschreibbare Herausforderungen stellen. Um dies zu erreichen, werden Fragen der folgenden Art gestellt: Welche kognitiven Prozesse werden durch die besondere Textkonfiguration in Anspruch genommen oder gezielt blockiert und welche eigenständigen Sinnkomplettierungen erlaubt der Text dem Leser im Sinne eines kohärenten Textverständnisses? In der vorliegenden Arbeit sollen zwei exemplarische Textanalysen dazu dienen, Hypothesen über die (ToM-relevanten) kognitiven Anforderungen der jeweiligen Textlektüre zu formulieren. Dabei werden mit Absicht zwei auf den ersten Blick deutlich unterschiedliche Texte gewählt. Dies ist durch die bisher offene Frage motiviert, ob die hohe Diversität literarischer Texte dazu führt, dass im Einzelfall der Lektüre unterschiedliche kognitive Funktionen angesprochen werden, oder ob sich die individuellen Strukturmerkmale der beiden Werke mit grundsätzlich ähnlichen Kognitionsprozessen in Verbindung bringen lassen.

(3) Reader-goal-Hypothesen

Die Eigenschaft literarischer Texte, die Attribuierungsleistung des Rezipienten in besonderem Maße zu fordern, ist laut Zunshine ein wichtiger Grund für einen von Lesern häufig vermeldeten Gratifikationseffekt. Der Einsatz hoher kognitiver Leistungen und die daraus resultierende Sinngenese vermitteln in diesem Sinne Freude und Zufriedenheit (vgl. Zunshine, 2006, S. 25). Daran anschließend stellt Zunshine die Hypothese auf, dass ein Grund für die Lektüre eines literarischen Textes in der Befriedigung ,kognitiver Gelüste (,cognitive cravings ${ }^{\circ}$ ) zu suchen ist (vgl. Zunshine, 2006, S. 4). Damit ist gemeint, dass mit der Lektüre literarischer Texte ein bestimmtes Ziel oder eine bestimmte Motivation des Lesers verbunden ist. Ein solches Lektüreziel wird z. B. dann erreicht, wenn ein Leser im Verlauf der Textlektüre in die Situation gebracht wird, bestimmte kognitive Ressourcen im Sinne eines gelingenden Textverständnisses nutzen zu müssen. „In other words, we do read novels because they engage our ToM [...]" (Zunshine, 2006, S. 164). Die Hypothese, dass mit der kognitiven Leistung während der Textlektüre ein bestimmtes Lektüreziel verfolgt 
wird, soll innerhalb dieser Dissertation nicht zur Diskussion stehen, da entsprechende Fragestellungen im Rahmen theoretischer Überlegungen nur schwer zu beantworten sind. Es bleibt einer empirischen Überprüfung vorbehalten zu belegen, ob die komplexen, durch den Rezipienten geleisteten Kognitionsprozesse, wie von Zunshine angenommen, tatsächlich einen Gratifikationseffekt auslösen.

(4) Evolutions- und Social-improvement-Hypothesen

Ausgangspunkt für Evolutions- und Social-improvement-Hypothesen ist die Annahme, dass die Lektüre literarischer Texte diejenigen kognitiven Mechanismen des Menschen aufruft, die ihre eigentliche evolutionäre Funktion in der Wahrnehmung und Handlungsplanung innerhalb nicht-ästhetischer Kontexte erfüllen. Es gilt die Annahme, dass literarische Texte evolutionär oder sozial relevante Probleme thematisieren, reflektieren oder den Leser zu evolutionär bzw. sozial relevanten Kognitionsleistungen animieren. Da literarische Texte auf diese Weise einen zusätzlichen Raum für kognitive Leistungen bieten, ist es denkbar, dass die Lektüre literarischer Texte einen Beitrag zur sozialen Entwicklung eines Individuums oder sogar der menschlichen Spezies leisten kann (Zunshine, 2006, S.16 f.). Hernadi schreibt zur evolutionären Funktion von Literatur:

Since prehistoric times literature has been serving two complementary functions: to expand the cognitive, emotive, and volitional horizons of human awareness and to integrate our beliefs, feelings, and desires within the fluid mentality required for survival in the complex social environments of human organisms. Frequent protoliterary transactions may have made some early humans more astute planners, more sensitive mind readers, and more reliable cooperators than their conspecific rivals, thereby increasing their chances to become the ancestors of contemporary men and women.

(Hernadi, 2002, S. 21)

Dem Forscherteam David C. Kidd und Emanuele Castano (2013, S. 377) gelang es, mit Blick auf eine mögliche adaptive Funktion der Lektüre literarischer Texte, mit Hilfe eines empirischen Versuchsdesigns einen Zusammenhang zwischen einer kurzfristigen Verbesserung der ToM-Fähigkeit des Menschen und der Lektüre literarischer Texte nachzuweisen. Wenn die Lektüre literarischer Texte neben dem realen sozialen Umfeld einen zusätzlichen Rahmen für die Aktivierung der relevanten kognitiven Ressourcen bildet, so die Annahme von Kidd und Castano, dann ließe sich dies mit Hilfe der Daten eines entsprechend gestal- 
teten Trainingsversuches darstellen. Dabei ist davon auszugehen, dass die häufige Konfrontation mit literarischen Texten die Fähigkeit zur Wahrnehmung mentaler Haltungen und Vorgänge im sozialen Umfeld begünstigt (vgl. Gallaghar \& Hutto, 2008). Aufbauend auf solchen Überlegungen formuliert Zunshine die folgende Schlussfolgerung: „Intense social species that we are, we thus read fiction because it engages, in a variety of particularly focused ways, our Theory of Mind“" (Zunsine, 2006, S. 162). Anstatt, wie Zunshine hier, die Frage zu beantworten, warum der Mensch fiktionale Literatur rezipiert, wird in der vorliegenden Arbeit der Versuch unternommen, eine Lösung für das Problem zu erarbeiten, wie die kognitive Architektur und insbesondere die mit dem Konzept der ToM in Verbindung stehenden kognitiven Ressourcen des Menschen mit den besonderen Merkmalen literarischer Erzähltexte in Interaktion treten. In diesem Rahmen sollen keine Rückschlüsse auf eventuelle adaptive Funktionen fiktionaler Literatur gezogen werden.

(5) Kontext-zentrierte kognitivistische Hypothesen

Kontext-zentrierte Hypothesen orientieren sich v. a. an kulturhistorischen Fragestellungen. Der Fokus liegt dabei auf den diachronischen Aspekten von Literatur im Kontext soziokultureller und historischer Entwicklungen. So kann z. B. nach relevanten historischen (medizinischen und philosophischen) Kognitionstheorien gefragt werden, die Einfluss auf die Entstehung oder Rezeption bestimmter Werke oder Werkgruppen nehmen. Alan Richardson untersucht z. B. den wechselseitigen Einfluss wichtiger Mimik-Theorien des 19. Jahrhunderts und einer Reihe literarischer Werke aus derselben Zeit (Richardson, 2010). Auch Lisa Zunshine untersucht in ihrem Beitrag Lying bodies of the enlightenment. Theory of Mind and cultural historicism kontext-orientierte Fragestellungen mit explizitem Bezug zum Konzept der ToM. Die Autorin beschäftigt sich hier mit der Darstellung von Körpersprache in ausgewählten Werken der Aufklärung (Zunshine, 2010). Ihr Ergebnis lautet, dass die in ihrem Beitrag besprochenen Autoren (im Gegensatz zu den Autoren anderer Epochen) ein besonderes Augenmerk auf das Potenzial der Körpersprache als Quelle für Hinweise auf innere Haltungen und Vorgänge legen: „Their particular achievement was to construct compelling social contexts in which whole spectrums of embodied performativity were present simultaneously, in the same narrative ,frame“, so to speak“ (ebd. S. 133).

Auch Kontext-zentrierte Fragestellungen stehen in der vorliegenden Arbeit nicht im Zentrum des Interesses. Um solche Fragen stichhaltig beantworten zu können, ist es notwendig, 
eine größere Menge individueller Texte miteinander zu vergleichen und zu ihrer gemeinsamen Zeitgeschichte (oder etwa zu einem Autor / einer Autorin) in Relation zu stellen.

\section{Aufbau und Vorgehensweise in dieser Arbeit}

Die Arbeit gliedert sich in drei Teile:

1. Nach dem einleitenden Teil wird das Kapitel II den kognitiv-psychologischen Hintergrund der ToM beleuchten. In diesem Arbeitsteil werden Forschungsergebnisse aus der kognitiven Entwicklungspsychologie zusammengetragen, um die Funktion der ToM für die soziale Interaktion präziser erfassen zu können. Anschließend wird die Frage gestellt, mittels welcher kognitiver Mechanismen, diese Fähigkeit funktioniert. Das Ziel ist es, die kognitiven Mechanismen, die mit der ToM in Verbindung stehen und sie ermöglichen, in übersichtlicher Form vorzustellen.

Das menschliche soziale Handlungsverständnis ist in der Vergangenheit auch im Rahmen sozialpsychologischer Studien ausführlicher besprochen worden. Dort liegt der Schwerpunkt auf der funktionalen Modellierung des menschlichen Verhaltens zum Zweck der Erfassung typischer sozialer Interaktionsformen in der Gruppe. Es steht dabei die Frage im Mittelpunkt, wie Menschen bestimmten Ereignissen, in diesem Fall dem Verhalten anderer, psychologische Ursachen zuschreiben und so ein Verständnis für die Handlungs- und Reaktionszusammenhänge in ihrem sozialen Umfeld gewinnen. Dabei wird die Attribuierung externer (im Sinne des dominanten Einflusses der äußeren Situation auf das Verhalten einer Person) und interner Faktoren (im Sinne des dominanten Einflusses mentaler Haltungen) gleichermaßen berücksichtigt. Besondere Aufmerksamkeit gilt den Ursachen und den Konsequenzen des menschlichen Verhaltens innerhalb einer sozialen Gruppe. Die Zuschreibung innerer Haltungen bildet dabei lediglich einen Teilbereich desjenigen sozialpsychologischen Modells, das den gesamten Komplex des sozialen Handlungs- und Situationsverständnisses erfassen soll. Auf Grund dieser weiten Perspektive bieten die Arbeiten der Sozialpsychologie keine detaillierten Einblicke hinsichtlich derjenigen kognitiven Prozesse, die die Funktionen der ToM im engeren Sinne steuern. 
Die ToM ist jedoch auch ein zentraler Forschungsgegenstand der kognitiven Entwicklungspsychologie. Die hier gewonnenen Erkenntnisse eignen sich besonders gut dafür, die einzelnen Funktionen der ToM detaillierter, als es in den bisherigen literaturwissenschaftlichen Arbeiten geschehen ist, aufzuschlüsseln. Das liegt u. a. daran, dass der Fokus der Entwicklungspsychologie nicht ausschließlich auf der allgemeinen Rolle der ToM in der sozialen Interaktion liegt. Viele Studien gelten darüber hinaus dem Ziel, genauer zu ergründen, wie - d. h. mit Hilfe welcher kognitiven Prozesse - die ToM funktioniert. Außerdem dienen die Ergebnisse der zahlreichen (zumeist empirischen) entwicklungspsychologischen Arbeiten als Grundlage dafür, zu bestimmen, für welche einzelnen Bereiche der sozialen Kognition die Fähigkeit zur Wahrnehmung und Zuschreibung innerer Vorgänge bedeutsam ist. Für die genauere Konzeptualisierung der ToM ist es an dieser Stelle daher hilfreich, die in den empirischen und theoretischen Studien dieses Faches gewonnenen Erkenntnisse zu Rate zu ziehen. Der Fokus der Entwicklungspsychologie liegt vor allem auf der (beobachtbaren) Entwicklung der entsprechenden Fähigkeiten im Kindesalter. Neben der genauen Funktionsweise der ToM sowie ihrer phylogenetischen und ontogenetischen Entwicklung wird außerdem die Frage nach dem Zusammenhang zwischen der ToM und anderen kognitiven Fähigkeiten wie z. B. den sprachlichen Kompetenzen und den sogenannten ,Exekutiven Funktionen` untersucht. Die Vielzahl (überwiegend empirisch) gewonnener Erkenntnisse aus den hier geleisteten Forschungsarbeiten gestattet einen Einblick in die Komplexität des Systems derjenigen kognitiven Mechanismen, die für die Wahrnehmung und Attribuierung mentaler Haltungen und Vorgänge notwendig sind.

Neben den empirischen entwicklungspsychologischen und sozialwissenschaftlichen Studien wird die genaue Funktionsweise des menschlichen Verständnisses mentaler Haltungen auch im Rahmen eines theoretischen Diskurses besprochen. Hier stehen die kognitiven Mechanismen der Verarbeitung unbeobachtbarer, innerer Vorgänge im Mittelpunkt des Interesses. Die wichtigsten in diesem Kontext besprochenen Ansätze sind die ,Theorie-Theorie‘, die ,Simulationstheorie‘ und die ,Narrativitäts-Theorie‘. Auch wenn die verschiedenen Thesen, die diesen theoretischen Ansätzen entspringen, bis heute zu keinem Konsens bezüglich der Funktionsweise der ToM geführt haben, finden sich auch hier Hinweise, die helfen können, das Verhältnis zwischen ToM und der kognitiven literarischen Rezeption besser zu verstehen. Aus diesem Grund sollen die grundlegenden theoretischen Ansätze zur Definition des ToM-Konzeptes im ersten Kapitel ausführlicher besprochen werden. 
Mit Hilfe der zusammengetragenen Erkenntnisse der empirischen ToM-Forschung und der relevanten theoretischen Diskussionen ist ein tiefergehendes Verständnis derjenigen kognitiven Vorgänge möglich, die der menschlichen ToM zugrunde liegen. Ziel dieses Arbeitsabschnittes ist es, mit Hilfe bereits vorliegender kognitionswissenschaftlicher Forschungsergebnisse ein detailliertes Bild der ToM-Fähigkeit des Menschen zu erstellen, das innerhalb literaturtheoretischer Überlegungen eine genauere Untersuchung des Bezuges zwischen den relevanten kognitiven Funktionen und den Merkmalen literarischer Texte ermöglicht. Es soll dabei nicht darum gehen, das Phänomen ToM neu zu konzipieren oder die Frage nach der Natur der kognitiven Mechanismen, die ihr unterliegen, erneut zu stellen. Vielmehr gilt es, sich aufbauend auf der besseren und umfassenderen Kenntnis der kognitiven Funktionen, die mit der ToM in Verbindung stehen, der Beantwortung der Frage nach ihrer grundlegenden Funktion im Zusammenhang mit der Lektüre literarischer Erzähltexte zu nähern.

2. Im Anschluss geht es in Kapitel III darum, den Zusammenhang zwischen zwei besonderen Bereichen der menschlichen Kognition - dem Verstehen literarischer Erzähltexte und der ToM - genauer zu erfassen. In einem ersten Schritt soll nach einem möglichen kognitiven ,common ground` zwischen den Kognitionsmechanismen der ToM und denjenigen der kognitiven Textrezeption gesucht werden. Die Aufgabe lautet zu klären, in welcher Relation die im ersten Kapitel beschriebenen Kognitionsmechanismen des Menschen zu den Kognitionsmechanismen der literarischen Rezeption stehen. Die vagen Ausdrücke ,Zusammenhang' und ,Relation“ sind momentan bewusst gewählt, da sich mögliche Gemeinsamkeiten oder sogar eine konstitutive funktionale Verbindung zwischen ToM und dem literarischen Textverstehen auf unterschiedliche Weise und auf unterschiedlichen Ebenen manifestieren können. Das Ziel dieses Arbeitsteils soll es sein, denkbare Berührungspunkte der beiden im Fokus stehenden Fähigkeiten (das Verstehen literarischer Erzähltexte einerseits und die ToM andererseits) vorzustellen. Darauf aufbauend gilt es, die Rolle der ToM-relevanten Kognitionsmechanismen im Zusammenhang mit der Rezeption literarischer Texte zu evaluieren. Damit ist die Vorgehensweise als tentativ einzustufen, denn es sind mehrere Hypothesen über die Art der Relation zwischen den ToM-relevanten Kognitionsmechanismen und den Mechanismen der literarischen Textrezeption denkbar, die besprochen werden sollen. Es ist z. B. möglich, dass die ToM und die literarische Textrezeption (a) grundsätzlich ähnliche Verstehensvorgänge bzw. Mechanismen der Informationsverarbeitung erfordern und 
daher überwiegend, vollständig oder zum Teil auf den gleichen kognitiven Mechanismen beruhen (Identitätsansatz). Um diese Annahme zu untermauern müssen sich strukturelle Parallelen in den Kognitionsanforderungen der beiden unterschiedlichen kognitiven Aufgaben aufzeigen lassen. Es ist ebenfalls denkbar, dass die Lektüre literarischer Texte zwar auf dem Einsatz von ToM-Mechanismen basiert, (b) diese aber in besonderem Ausmaß oder in besonderer Form im Vergleich zur Verarbeitung realer sozialer Interaktionen fordert (Stimulationsansatz). Das wäre dann der Fall, wenn literarische Texte zwar mit Hilfe der Kognitionsmechanismen der ToM zu verstehen sind, aber aufgrund ihrer spezifischen Merkmale besondere - von Alltagssituationen abweichende Ansprüche an dieselben Informationsverarbeitungsprozesse stellen. Es ist drittens denkbar, dass die Kognitionsmechanismen der ToM eine notwendige Voraussetzung für das Verständnis literarischer Erzähltexte sind (Voraussetzungsansatz). In einem solchen Fall wären Argumente dafür zu finden, dass die literarische Rezeption - so wie wir sie kennen - ohne die Kognitionsmechanismen der ToM nicht möglich wäre.

Um diese drei Ansätze bewerten zu können, ist es zunächst wichtig zu klären, ob sich die These, dass Menschen ihre kognitiven Mechanismen im Zuge der Lektüre eines literarischen Textes in analoger Form zu realen Situationen nutzen können, begründen lässt. Dies ist vor allem deshalb notwendig, weil diese intuitive Annahme in den bisher geleisteten literaturtheoretischen Arbeiten, die sich mit diesem Thema befassen als wahr vorausgesetzt, jedoch nicht näher geprüft wird. Anschließend sollen die möglichen Funktionen der intersubjektiven Verstehensleistungen, die dank der ToM möglich sind, in einen Bezug zu den Verstehensleistungen im Zuge der literarischen Textrezeption gestellt werden. Aufbauend auf die These, die ToM könne im Zuge der Textrezeption in vergleichbarer Form zugunsten von Sinngebungs- und Kohärenzschaffungsprozessen genutzt werden, wie im Rahmen realer sozialer Interaktion, ist zu prüfen, ob der ToM für die kognitive literarische Rezeption nicht eine weitaus bedeutsamere Rolle zufällt, als ihr bereits zugesprochen wurde. Die Funktion der ToM für die literarische Textrezeption ist m. E. mit einem Blick auf die Interpretation literarisch dargestellter Handlungen oder die (intentionalen) Handlungen von Autoren nicht erschöpfend beschrieben. Vielmehr ist es denkbar, dass die ToM - also das Konglomerat kognitiver Leistungen, das die Fähigkeit zur Wahrnehmung und Attribution mentaler Haltungen und Vorgänge begründet - auch mit den strukturellen Eigenschaften eines Textes und allgemei- 
nen Faktoren wie z. B. Merkmalen der Literarizität und Fiktionalität in einem funktionalen Zusammenhang steht. Um herauszufinden, ob und für welche Textmerkmale dies zutrifft, gilt es die besondere Beschaffenheit eines literarischen Erzähltextes mit den bereits im ersten Teil der Arbeit zusammengestellten kognitiven Funktionen und Mechanismen der ToM abzugleichen.

Eine Untersuchung der Relation zwischen Textmerkmalen und Kognitionsmechanismen unter dieser Prämisse betrifft den top-down orientierten Rezeptionsprozess. Dabei geht es darum, die Funktion der dem Menschen gegebenen kognitiven Ressourcen (in diesem Fall mit einem besonderen Fokus auf der ToM) im Rahmen einer bestimmten Tätigkeit - hier der Lektüre eines literarischen Erzähltextes - zu erhellen. Dies lässt sich realisieren, indem einzelne Merkmalsgruppen literarischer Erzähltexte aufgefächert und daraufhin geprüft werden, welche Parameter sie für die kognitiven Verstehensprozesse eines Lesers darstellen. In diesem Zusammenhang ist es notwendig, Forschungsliteratur aus der literaturtheoretischen Forschung zu Rate zu ziehen, die Auskunft über die Charakteristika solcher Texte gibt. Mit Hilfe der dort zusammengetragenen Erkenntnisse soll nach Schnittstellen gesucht werden, die eine Verarbeitung literarischer Informationen mit Hilfe kognitiver Mechanismen, die mit der ToM in Verbindung stehen, wahrscheinlich oder notwendig machen.

Eine systematische Analyse, insbesondere spezifisch literarischer Eigenschaften (v. a. hinsichtlich der strukturellen Merkmale eines literarischen Erzähltextes) und ihrer möglichen Beziehungen zu den erforderlichen Kognitionsleistungen des Lesers, steht bis heute aus. Dabei ist es wichtig, an Hand einer genauen Betrachtung einzelner Merkmalsgruppen literarischer Erzähltexte zu zeigen, auf welchen Ebenen des literarischen Rezeptionsvorganges die ToM-relevanten kognitiven Mechanismen eingebunden sind. Meine Annahme lautet diesbezüglich, dass die ToM-relevanten kognitiven Ressourcen nicht nur für die Attribuierung mentaler Haltungen hinsichtlich fiktiver Figuren oder anderer anthropomorpher Einheiten (die auch das Verständnis von Attribuierungsvorgängen der Figuren und der Erzählinstanz untereinander einschließt) eingesetzt werden. Sie sind vielmehr auch mit weiteren Momenten der literarischen Textrezeption verbunden, die es zu identifizieren gilt. Um dies umfassend darzustellen, gilt es den Blick von der inhaltlichen Ebene literarischer Texte zu lösen und nach den möglichen Funktionen einzelner kognitiver Mechanismen zu suchen, die sich mit der besonderen Beschaffenheit eines literarischen Erzähltextes in einen Zusammenhang stellen lassen. Dafür ist es 
notwendig, Konzepte und Begriffe aus der literaturtheoretischen Forschung zu Rate zu ziehen. Es ist zu klären, ob sich mit Hilfe einer solchen Vorgehensweise stichhaltige Rückschlüsse auf die Funktion der ToM-relevanten kognitiven Ressourcen ziehen lassen, die für die kognitive Verarbeitung der entsprechenden Texteigenschaften notwendig sind. Im Zuge dessen muss geprüft werden, ob es mit Hilfe der im ersten Kapitel geleisteten Aufschlüsselung der einzelnen kognitiven Mechanismen der ToM nun möglich ist, die Funktion(en) verschiedener einzelner kognitiver Vorgänge im Rahmen der kognitiven Textrezeption sinnvoll zu bewerten. Ist dies der Fall, und lassen sich gleichzeitig konstitutive Parallelen zwischen den kognitiven Mechanismen der ToM und der Textrezeption aufzeigen, dann liegt der Schluss nahe, dass die beiden hier untersuchten Tätigkeiten auf der Ebene kognitiver Verarbeitungsprozesse essenziell miteinander ,verwandt' sind.

3. Neben der Zentralität der ToM-relevanten kognitiven Mechanismen für die literarische Rezeption im Allgemeinen darf die Möglichkeit nicht außer Acht gelassen werden, dass die besonderen strukturellen Eigenschaften eines literarischen Erzähltextes den Leser potenziell mit besonderen Anforderungen für die Informationsverarbeitung konfrontieren. Es scheint in dieser Hinsicht durchaus wahrscheinlich, dass die spezifischen Merkmale eines literarischen Erzähltextes einen entscheidenden Einfluss auf die kognitive Informationsverarbeitung nehmen, indem sie insbesondere die Informationsaufnahme während der Lektüre strukturieren. Wenn ein literarischer Erzähltext als Informationssystem zu betrachten ist, das auf Grund seiner spezifischen Konfiguration die kognitiven Rezeptionsprozesse des Lesers ,dirigiert', dann stellt sich die Frage, ob sich die Bedingungen für diese Kognitionsprozesse mittels einer methodischen Textanalyse genauer charakterisieren lassen. Um diese Möglichkeit auszuloten, ist es hilfreich, spezifische Textkonfigurationsformen zu benennen und diese anschließend mit den relevanten Kognitionsprozessen in einen Zusammenhang zu stellen. Das Kapitel IV ist dem Versuch gewidmet, das Wirkungspotenzial literarischer Texte an Hand zweier exemplarisch ausgewählter Textanalysen zu untersuchen.

Da die individuellen Unterschiede literarischer Texte untereinander an dieser Stelle im Zentrum des Interesses stehen, ist es sinnvoll, zwei Werke miteinander zu vergleichen, die sich durch eine hohe semantische und strukturelle Differenz auszeichnen. Diese Vorgehensweise ist ebenfalls durch den Anspruch gerechtfertigt, die mögliche Bandbreite der Interaktionsmechanismen zwischen relevanten kognitiven Funktionen und den jeweiligen Textmerkmalen aufzuzeigen. Die Wahl fällt in dieser Arbeit auf die 
Njáls Saga (13. Jh.), eine der größten und wichtigsten Islandsagas, und den postmodernen deutschsprachigen Erzähltext Gehen (1972) von Thomas Bernhard. Die Textanalysen sollen Raum dafür bieten, den für das jeweilige Werk hervorstechenden Merkmalen zu folgen und so eine Vorstellung von den besonderen kognitiven Anforderungen zu gewinnen, die der einzelne Text an seinen Leser stellt. Die in diesem Arbeitsteil angestellten Überlegungen sollen zeigen, dass nicht nur die Beachtung typischer, sondern auch der Einbezug individueller Merkmale literarischer Erzähltexte sinnvoll ist, um die besondere Rolle der ToM-relevanten kognitiven Funktionen für die kognitive literarische Rezeption zu erhellen.

Die Textbeispiele sind - in Abgrenzung zu Zunshines Textanalysen - so gewählt, dass sie mentale Attribuierungsvorgänge bzw. die Themen der Wahrnehmung und Kognition nicht, oder nicht auf den ersten Blick, darstellen. Um sowohl semantische als auch strukturelle Texteigenschaften systematisch und übersichtlich erfassen zu können, erfolgt die Analyse der beiden Erzähltexte hauptsächlich mit den Mitteln der Narratologie. Die narratologische Analyse dieser zwei Werke bildet hier die Grundlage, um den genauen Zusammenhang zwischen den Merkmalen des einzelnen Textes und den Kognitionsprozessen des Lesers zu beschreiben. Dafür sind diejenigen individuellen Eigenschaften des jeweiligen Werkes zusammenzustellen, von denen begründet angenommen werden kann, dass sie Einfluss auf den jeweiligen kognitiven Rezeptionsprozess nehmen. Es sei damit nicht die Behauptung aufgestellt, dass diese Vorgehensweise die einzige Methode darstellt, um das Verhältnis zwischen Textkonfiguration und kognitiver Rezeption kenntlich machen zu können. Allerdings wird zu zeigen sein, dass mit ihr eine mögliche Methodik zur Verfügung steht, um mögliche Wirkungsmechanismen an der Schnittstelle zwischen Leserkognition und Textmerkmal aufzudecken. 



\section{Was ist ,Theory of Mind‘?}

\section{Begriffsbestimmung}

Eines der Vorhaben dieses Forschungsprojektes liegt in der Ausleuchtung der vielfältigen Aufgaben der ToM innerhalb des kognitiven literarischen Rezeptionsprozesses. Um sich diesem Ziel anzunähern, ist es unerlässlich, zunächst das Konzept der ToM selbst genauer zu betrachten. Darauf aufbauend lässt sich dann die Tragweite der damit assoziierten kognitiven Funktionen in ihrer jeweiligen Rolle während der Lektüre literarischer Texte bestimmen. Die Frage, die es in diesem einführenden Abschnitt also zu beantworten gilt, lautet: Was ist die ToM genau und auf welchen kognitiven Mechanismen basiert diese menschliche Fähigkeit?

Der Begriff ,Theory of Mind“ wurde durch einen Artikel der beiden Psychologen David Premack und Guy Woodruff (1978) geprägt, die sich dort die Frage stellten: „Does the chimpanzee have a theory of mind?“ (Premack \& Woodruff, 1978). Mit der Wahl ihrer Terminologie lenkten sie den Fokus der Kognitionsforschung nicht nur auf die Rolle der Wahrnehmung und Attribution mentaler Haltungen in sozialen Interaktionen, sondern prägten gleichzeitig die Vorstellung der Art der kognitiven Prozesse, die für eine funktionale ToM erforderlich sind. Ihre Definition des Begriffes lautet wie folgt:

In saying that an individual has a theory of mind, we mean that the individual imputes mental states to himself and to others (either to conspecifics or to other species as well). A system of inferences of this kind is properly viewed as a theory, first, because such states are not directly observable, and second, because the system can be used to make predictions, specifically about the behavior of other organisms. (Ebd., S. 515)

Die Fähigkeit nicht direkt einsehbare mentalen Haltungen und Vorgänge wie Intentionen, Wünsche, Überzeugungen, Wissen und Gefühle zu attribuieren ist eine wichtige Grundlage 
dafür, die Handlungen anderer innerhalb komplexer sozialer Gefüge interpretieren, antizipieren und manipulieren zu können. Wenn wir jemanden glücklich machen wollen, müssen wir die Bedürfnisse und Wünsche dieser Person kennen. Wenn wir in einer Konkurrenzsituation bestehen wollen, müssen wir die Intentionen anderer, die mit den eigenen kollidieren, richtig einschätzen. Nur dann können wir unser eigenes Handeln entsprechend zu unserem Vorteil anpassen. Um jemandem einen Sachverhalt verständlich zu machen und dabei möglichst effizient kommunizieren zu können, ist es wichtig, den bisherigen Wissens- oder Kenntnisstand des Gegenübers möglichst genau einzuschätzen. Dies sind nur wenige Beispiele, die illustrieren, dass die ToM in vielen unterschiedlichen sozialen Situationen hilfreich oder gar notwendig ist, um diese Situationen korrekt bewerten und sich angemessen verhalten zu können. Besonders deutlich tritt die Relevanz der ToM für den alltäglichen sozialen Umgang im Fall von Kindern in Augenschein, die an Entwicklungsstörungen wie zum Beispiel der Autism Spectrum Disorder (ASD) leiden. In ihrem Fall ist auffällig, dass spezifisch diejenigen kognitiven Mechanismen beeinträchtigt sind, die der ToM zuzurechnen sind. Die Fähigkeit zur kognitiven Konzeptualisierung innerer Vorgänge ist bei ihnen nicht oder nur eingeschränkt ausgebildet. Genau aus diesem Grund, so der Konsens der ASD-Forschung, haben betroffene Kinder große Probleme, sich in komplexen sozialen Situationen zu orientieren und mit anderen Menschen zu interagieren. ${ }^{8}$

In empirischen Studien, die pragmatisch orientierten Fragestellungen (etwa: ,Was kann ein Mensch dank der ToM im sozialen Kontext tun bzw. verstehen?') nachgehen, lassen sich neben der generellen Relevanz der ToM für die soziale Interaktion auch einzelne unterschiedliche Kognitions- und Verstehensleistungen voneinander abgrenzen. Dabei steht die Frage im Fokus, ob die Versuchsteilnehmer unterschiedliche konstruierte soziale Interaktionsszenarien, für deren Verständnis die Attribuierung mentaler Haltungen und Vorgänge ausschlaggebend ist, richtig interpretieren. Diese Studien werden oft mit Kindern aus verschiedenen Altersgruppen durchgeführt, um die Entwicklung der ToM nachzuzeichnen bzw. das Vorhandensein oder Nicht-Vorhandensein der ToM bei Kindern unterschiedlichen Alters zu demonstrieren.

Die Testdesigns dieser Studien variieren häufig in wichtigen Details. So stehen den Versuchspersonen zum Teil unterschiedlich detaillierte Informationen für eine Situationsein-

\footnotetext{
${ }^{8}$ Eine detaillierte Untersuchung des Zusammenhangs zwischen der Entwicklung von Fähigkeiten, die mit der ToM assoziiert sind und ASD findet sich z. B. bei Hoogenhout \& Malcolm-Smith (2014).
} 
schätzung über die konstruierten Szenarien zur Verfügung. Außerdem können sich die Fragestellungen auf unterschiedliche Kategorien mentaler Haltungen (z. B. Intentionen oder Überzeugungen) beziehen. Um einen systematischen Überblick über die Vielzahl der gesammelten Daten zu gewinnen, erstellten Wellman und Liu im Jahr 2004 eine Metastudie, in deren Rahmen zahlreiche bereits abgeschlossene Testparadigma statistisch ausgewertet und miteinander verglichen werden (Wellman \& Liu, 2004). Dabei ließen sich eine Reihe signifikanter Alterseffekte nachweisen. Das bedeutet, dass einige ToM-Tests von Kindern jüngeren Alters zuverlässig bestanden werden, andere jedoch erst ab einer höheren Altersgruppe. Die statistischen Daten aus den einzelnen Studien erlaubten es Wellmann und Liu, unterschiedliche Kompetenzen des sozialen Umganges, die die Attribuierung mentaler Haltungen voraussetzen, in eine Relation zueinander zu stellen. Sie schreiben: „No single type of task - for example, false-belief tasks - can adequately capture this developmental progression. Similarly, no theory will be adequate that does not account for these various, developmentally sequenced acquisitions" (ebd. S. 537). Wellman und Liu stellten in diesem Zusammenhang fest, dass sich die Testdesigns, die jeweils unterschiedliche Kategorien mentaler Haltungen abfragten, untereinander hierarchisieren ließen. Auf diese Weise wird ein konsekutiver Entwicklungszusammenhang der einzelnen sozialen Kompetenzen, die die Fähigkeit zur Wahrnehmung und Attribution innerer Vorgänge erfordern, sichtbar. Wellmann und Liu erstellten darauf aufbauend die sogenannte ,ToM-Skala', die die Entwicklung ToMrelevanter Kompetenzen gesunder Kinder in Relation zu ihrem Alter abbildet. ${ }^{9}$ Diese Skala enthält fünf aufeinander aufbauende Entwicklungsziele. Mit zunehmendem Alter lernen Kinder 1. unterschiedliche Wünsche und Bedürfnisse zu erkennen (diverse desires), 2. verschiedene Überzeugungen voneinander zu unterscheiden (diverse beliefs), 3. den gegenüber dem eigenen limitierten bzw. erweiterten Zugang zu Wissen zu kalkulieren (knowledge access), 4. falsche Überzeugungen zu dekodieren (false belief) und 5. Emotionen korrekt vorherzusehen bzw. von vorgetäuschten falschen Emotionen zu unterscheiden (belief emotion / real vs. apparent emotion). Ist eine Stufe dieser Skala einmal erklommen, sind gesunde Kinder immer in der Lage, niedriger angesiedelte Aufgabenstellungen zuverlässig zu lösen.

Die ToM-Skala erfasst die Entwicklung kognitiver Konzepte hinsichtlich unterschiedlicher mentaler Haltungen, wie Intentionen, Überzeugungen, Emotionen usw. von gesunden Kindern. Sie ist ein geeignetes Mittel, um wichtige Entwicklungsschritte von Kindern hinsicht-

\footnotetext{
${ }^{9}$ Diese Ergebnisse bestätigten und bekräftigen die Ergebnisse einer früheren Meta-Analyse von einer Reihe empirischer Studien, durchgeführt von Wellman, Cross \& Watson (2001).
} 
lich der ToM und den gestaffelten Leistungsanspruch des Verstehens unterschiedlicher mentaler Konzepte wie ,Überzeugung', ,Wunsch“ oder ,Wissen` sichtbar zu machen. Allerdings fehlen unter den auf dieser Skala erfassten Kategorien einige Fähigkeiten, für die ebenfalls die Fähigkeit zur kognitiven Verarbeitung oder Attribution innerer Vorgänge und mentaler Haltungen notwendig ist. Gemeint sind hier 1. das grundlegende Verständnis von Intentionalität und zielgerichtetem Handeln, 2. das kindliche Fiktionsspiel (pretend play) (z. B. Lillard, 1993 $)^{10}$, 3. die Koordination der eigenen Wahrnehmung mit derjenigen eines anderen (joint attention) (vgl. z. B. Charman, Baron-Cohen, Swettenham, Baird, Cox \& Drew, 2000). 4. das Entschlüsseln komplexer mentaler Zusammenhänge, wie z. B. eingebettete Überzeugungen oder Intentionen höherer Ordnung (A bezweifelt, dass B weiß, dass C überzeugt ist, etwas sei der Fall) und 5. das Verstehen uneigentlicher Kommunikation (z. B. Lügen oder ironischer Äußerungen) (vgl. z. B. Winner, Happé, Blum \& Pincus, 1998). Das folgende Fazit von Wellman und Liu fasst die Erkenntnisse der ToM-Forschung treffend zusammen: „[...] developing a theory of mind includes understanding multiple concepts acquired in an extended series of developmental accomplishments“ (Wellman \& Liu, 2004, S. 523). Alle oben genannten Verstehensleistungen müssen Beachtung finden, wenn die Rolle der ToM in der Rezeption literarischer Texte genauer untersucht werden soll. Es reicht im Zuge eines solchen Vorhabens nicht aus, die ToM lediglich in allgemeiner Form als ,Fähigkeit sich selbst und anderen mentale Haltungen zuzuschreiben` zu definieren, da diese allgemeine Definition keine Einsicht in die Funktionsweise dieser Fähigkeit bietet. Vielmehr ist ein genauerer Blick auf die kognitiven Leistungen, die sie ermöglichen sinnvoll. Diese Leistungen geben Hinweise auf diejenigen Mechanismen, die die ToM ermöglichen. In der anschließenden rezeptionstheoretischen Untersuchung kann dann an Hand dieser Mechanismen und nicht nur basierend auf der allgemeinen Definition der ToM nach Zusammenhängen mit literarischen Merkmalen gesucht werden. Die Versuchsanordnungen der kognitiven Entwicklungspsychologie, die für die Untersuchung der einzelnen Kategorien mentaler Haltungen erstellt wurden, dienen bei der Suche nach diesen Mechanismen als Orientierung. Diese Testdesigns lassen Rückschlüsse auf die kognitiven Anforderungen zu, die im Rahmen der einzelnen Aufgabenstellungen für die Testteilnehmer bestehen. Mein Ziel ist es, insgesamt eine genauere Vorstellung von den einzelnen kognitiven Leistungen zu gewinnen, die es uns Menschen ermöglichen, ,eine ToM zu besitzen‘. Auf diese Weise wird eine geeignete

\footnotetext{
${ }^{10}$ Hier steht im Besonderen der Zusammenhang zwischen ToM und pretend play im Fokus der Untersuchung.
} 
Grundlage dafür gelegt, um die Funktion(en) der ToM, die ihr im Zuge der kognitiven Rezeption literarischer Texte zufallen, besser als bisher zu verstehen. Um dies zu erreichen, sind zwei Arbeitsschritte notwendig. Aufbauend auf die allgemeine kognitionspsychologische Definition der ToM als ,Fähigkeit, sich und anderen mentale Haltungen zuschreiben zu können', möchte ich zunächst eine Reihe entwicklungspsychologischer Erkenntnisse über unterschiedliche soziale Verstehensleistungen zusammentragen, für die die Attribution und das Verständnis mentaler Haltungen relevant ist. In diesem Kontext wird deutlich, dass die ToM in unterschiedlichen Bereichen der sozialen Interaktion eine Rolle spielt und daher unterschiedliche Verstehensleistungen beinhaltet. Mit diesen Leistungen sind z. B. das Verständnis unterschiedlicher mentaler Konzepte (,Überzeugung‘ vs. ,Wunsch`, vs. ,Wissen“ etc.), die Fähigkeit zur Koordination fremder und eigener Aufmerksamkeit (,joint attention ‘) und das kindlichen Fiktionsspiel (,pretend play`) u. Ä. gemeint. Wenn gesagt wird, dass gesunde Erwachsene ,über eine ToM verfügen', dann ist also gemeint, dass sie eine Reihe von Verstehensleistungen erbringen können, die mit dem Verständnis und der Attribution mentaler Haltungen und innerer Vorgänge zusammenhängen. Damit ist noch nicht erklärt, wie - d. h. mit Hilfe welcher kognitiven Prozesse und Mechanismen - die ToM funktioniert. Die Frage nach der kognitiven Funktionsweise der ToM muss also ebenfalls gestellt werden, um herauszufinden, welche kognitiven Prozesse und Mechanismen notwendig die ToM-relevanten sozialen Leistungen gesunder Erwachsener ermöglichen.

\section{Die unterschiedlichen Funktionen der ToM im Kontext sozialer Interaktion}

\subsection{Einführung: Die Interpretation psychologisch motivierter Handlungen}

Menschliche Handlungen basieren nicht allein auf den sichtbaren Gegebenheiten einer sozialen Umgebung. Sie resultieren auch aus den nicht direkt einsehbaren inneren Haltungen und Vorgängen der miteinander interagierenden Individuen. Um eine fremde Handlung verstehen zu können, muss ein Beobachter beide Elemente - sichtbare Gegebenheiten der sozialen Umgebung und anzunehmende innere Haltungen - in einen kausalen Zusammenhang zueinander stellen. Solche handlungsmotivierenden inneren Haltungen sind z. B. Intentionen 
(intention), Wünsche (desire) und Überzeugungen (belief). Sie äußern sich z. T. in beobachtbaren körperlichen Regungen, Bewegungen, Gestik und Mimik. Das klingt zunächst einmal selbstverständlich, allerdings widersprechen beobachtbare Handlungen häufig den real gegebenen Umständen - ein Mensch greift bspw. zum Regenschirm, obwohl die Sonne scheint. Viele Handlungen erscheinen erst dann sinnvoll, wenn ein entsprechender mentaler Vorgang zugeordnet werden kann. Die Differenzierung dieser beiden Komponenten von menschlichen Handlungen ist auch deshalb wichtig, weil die gleiche sichtbare Bewegung in zwei verschiedenen Kontexten durch unterschiedliche mentale Haltungen ausgelöst worden sein kann. So lässt sich ein ausgestreckter Zeigefinger sowohl als Zeigegeste als auch als Einforderung von Aufmerksamkeit interpretieren. Ein Mensch, der in der Lage ist, die beobachteten Aktionen als zielgerichtet und psychologisch motiviert wahrzunehmen und die ihnen zu Grunde liegenden Intentionen, Wünsche oder Überzeugungen des Gegenübers korrekt zu entschlüsseln, kann eine gegebene Situation optimal einschätzen und die eigenen Handlungen gegebenenfalls in adäquater Form anpassen. Um ein solches Handlungsverständnis zu ermöglichen ist ein Verständnis der funktionalen Eigenschaften von unterschiedlichen Kategorien mentaler Haltungen notwendig. Es ist also wichtig, zu wissen, was eine Überzeugung über die Welt von Wissen über die Welt unterscheidet und in welchem funktionalen Verhältnis Wünsche und Intentionen zu menschlichen Handlungszielen stehen. Wenn ein Mensch diese Kompetenzen besitzt, ist es ihm möglich, Handlungen nicht lediglich auf die sichtbaren Gegebenheiten einer Situation zurückzuführen, sondern sie mit Hilfe der Attribution von mentalen Haltungen zu interpretieren. Solche Interpretationen basieren dabei häufig auf einer Kombination einzelner Kategorien mentaler Haltungen und den uns zugänglichen Informationen über eine gegebene Situation. Die Handlungen einer Person, die durch einen Bahnhof eilt, verstehen wir, wenn wir ihr zuschreiben, dass sie einen Zug erreichen möchte (desire), allerdings davon überzeugt ist (belief), diesen Zug verpassen zu können. Sie beschleunigt daher ihre Schritte, um den Zug dennoch zu erreichen (intention). Die Entwicklung der Fähigkeiten unterschiedliche mentale Haltungen zuzuschreiben, lässt sich an Hand von Studien mit Vorschulkindern unterschiedlicher Altersstufen nachvollziehen. 


\subsection{Einzelne Dimensionen der Handlungsmotivierung}

\section{Intentionen}

Kinder zeigen schon sehr früh in ihrer Entwicklung (in einem Alter ab 5 Monaten) ein Verständnis für zielgerichtetes intentionales Handeln, wie eine Habituationsstudie von Amanda Woodward zeigt (Woodward, 1998). Für Woodwards Studie wurden die Kinder auf ein bestimmtes Ereignis habituiert. Das heißt sie sahen einen menschlichen Arm auf einer Puppenbühne immer wieder nach einem bestimmten von zwei nebeneinander platzierten Objekten (z. B. einem Ball oder einem Teddybären) greifen. In der anschließenden Testphase sah ein Teil der Kinder die Hand nach demselben Objekt greifen, das jedoch mit dem anderen Objekt den Platz getauscht hatte (new path-Gruppe), ein weiterer Teil der Kinder sah den Arm dieselbe Bewegung vollführen, dafür aber nach dem getauschten Objekt greifen (new object-Gruppe). Die Blickzeiten der zweiten Gruppe erwiesen sich als deutlich länger als diejenigen der ersten Gruppe. Diese stärkere Reaktion auf das neue Objekt lässt sich laut Woodward damit erklären, dass die Kinder dem menschlichen Agenten zuvor die Intention zugesprochen hatten, den Ball zu greifen. Der Intentionswechsel der new-object-Situation stellt damit für die Kinder ein neues Ereignis dar, so dass die während der Habituationsphase verkürzten Blickzeiten nicht mehr gelten. Der Griff nach einem anderen Objekt eröffnet offenbar einen neuen kognitiven Vorgang und induziert aus diesem Grund erneut verlängerte Blickzeiten seitens der Kinder.

Neben Studien, in denen ein Habituationstestdesign gewählt wurde, nutzen verschiedene andere Testdesigns das angeborene kindliche Imitationsverhalten zur Testung ihrer Hypothesen. Auch diese Studien bestätigten die wichtigsten Hypothesen der ersten Versuche und dokumentieren damit ebenfalls die Orientierung schon sehr junger Kinder an Hand angenommener Intentionen oder Ziele. In einer solchen Studie zeigte sich beispielsweise, dass Kinder im Alter von 7 Monaten ihr Imitationsverhalten den zugeschriebenen Zielen und Intentionen der beobachteten Person anpassten. Die Kinder imitierten in diesem Zusammenhang überzufällig häufig die zielgerichteten Aspekte einer beobachteten Handlung (Hamlin, Hallinan \& Woodward, 2008). Dieser Hinweis auf das frühkindliche Handlungsverständnis belegt die frühe Sensitivität für Zielgerichtetheit, die bereits innerhalb des ersten Lebensjahres vorhanden ist, eindrücklich. Ältere Kinder (18 Monate) beherrschen die Entschlüsselung von Intentionen bereits schon so weit, dass sie beobachtete Handlungen eher gemäß den angedeuteten Intentionen nachahmen als sie nur zu kopieren. Andrew Meltzoff zeigte seinen jungen Testteilnehmern Handlungen, die zwar auf ein Ziel ausgerichtet waren, jedoch vor 
ihrer vollständigen Ausführung unterbrochen wurden und stellte fest, dass die Kinder zumeist die vollständige intendierte und nicht ausschließlich die gesehene Handlung imitierten (Meltzoff, 1995). Das bedeutet, dass es Anderthalbjährigen bereits möglich ist, Intentionen von gesehenen misslungenen Handlungen abzuleiten, ohne eine vollständig ausgeführte Handlung zuvor beobachtet zu haben.

\section{Kommunikationsintentionen}

Die Attribution von Intentionen ist nicht nur im Zuge der Interpretation menschlicher Handlungen relevant. Auch unsere Kommunikation basiert weitgehend auf diesen Fähigkeiten. Dies wird im Fall uneigentlicher Kommunikationsformen besonders deutlich. Die Fähigkeit, verschiedene Formen uneigentlicher Kommunikation zu verstehen, entwickelt sich in einem Alter von 6 bis 8 Jahren. Sie baut auf der Fähigkeit auf, beobachtetes Verhalten auf Intentionen zurückführen zu können (Perner, 1999, S. 217). Anders als im Fall direkter und unverschlüsselter Rede basiert ein gelingender Kommunikationsakt, der Formen der uneigentlichen Kommunikation beinhaltet, nicht allein auf dem Verstehen des buchstäblichen Wortsinns, sondern auf der Dekodierung einer uneigentlichen Sinnebene. Die Liste der Kommunikationsformen, bei denen eine solche implizite oder nicht direkt benannte Sinnebene für das Verständnis der Aussage relevant ist, umfasst unter anderem (unabsichtliche) Fehler, Lügen, Witze, Ironie, Heuchelei, Neckereien, Understatements, Übertreibungen und Metaphern. Diese verschiedenen Redeformen können laut Susan R. Leekam (1991, S. 160) mit Hilfe eines Modells der ihnen zugrunde liegenden mentalen Haltungen auf Seiten des Sprechers und des jeweiligen Adressaten dargestellt werden. Sie schlägt eine Klassifikation auf zwei Ebenen vor: Auf der ersten Ebene unterscheidet sie die intendierte von der nicht intendierten uneigentlichen Rede, wobei in die zweite Kategorie ausschließlich unbewusste Falschaussagen fallen. Um solche Sprechakte zu entschlüsseln, muss der Adressat lediglich verstehen, dass der Sprecher sich der Falschheit seiner Äußerung nicht bewusst ist und es seine eigentliche Absicht (Intention) ist, eine wahre Aussage zu treffen. Für alle anderen Arten uneigentlicher Rede muss der Adressat eine höhere kognitive Leistung erbringen und - nachdem er zwischen den zwei Varianten der intendierten oder nicht intendierten Falschheit der Aussage entschieden hat - anschließend den semantischen Gehalt der eigentlich intendierten Aussage bestimmen. Wie zuvor eröffnen sich auch hier wieder zwei grundsätzliche Möglichkeiten: Zum einen kann es (wie z. B. bei einer Lüge) in der Absicht des Sprechers liegen, den Adressaten zu täuschen, also über einen wahren Sachverhalt im Dunkeln zu lassen und damit dessen mentale Haltung zu manipulieren. Zum anderen ist es denkbar, 
dass er lediglich einen zu kommunizierenden Sachverhalt durch eine ironische Äußerung unterstreichen möchte. In beiden Fällen (Lüge und Ironie) ist ein Verständnis der betreffenden Aussagen ohne die Fähigkeit, einem Gegenüber mentale Haltungen zuzuschreiben, nicht möglich.

Eine Täuschungsabsicht, die zum Beispiel im Fall einer Lüge vorliegt, lässt sich als wissentliche Evokation oder Manipulation einer mentalen Haltung (bspw. einer Überzeugung) mit Hilfe einer bewussten Falschaussage definieren. Derjenige, der diese Lüge entweder beobachtet oder sich selbst in den Schuhen des Belogenen wiederfindet, müsste, um die Lüge zu enttarnen, den Widerspruch zwischen einem kommunizierten und dem tatsächlichen Sachverhalt erkennen. Dies sei zu Gunsten besserer Anschaulichkeit an Hand eines Szenarios mit drei Personen illustriert: Von den drei Personen A, B und C sind zwei aktiv an dem Kommunikationsakt beteiligt - A und B - während C einen unbeteiligten Beobachter darstellt. Die Variable X soll für einen unwahren Sachverhalt (z. B. ,Coca-Cola enthält keinen Zucker') stehen. A behauptet nun gegenüber B, X sei der Fall, obwohl in Wirklichkeit X nicht der Fall ist. Im Fall einer Täuschungsabsicht umfassen die mentalen Haltungen von A dabei das Wissen, dass $\mathrm{X}$ nicht der Fall ist, die Einschätzung des Wissensstandes von $\mathrm{B}$ gegenüber X und die Intention B in den Glauben zu versetzen, X sei der Fall. Voraussetzung für eine gelungene Täuschung ist dabei, dass B nicht weiß, dass $X$ in Wirklichkeit nicht der Fall ist, und davon ausgeht, A verfolge die Intention die Wahrheit zu sagen. Davon ausgehend, dass C, der den Kommunikationsakt beobachtet, bekannt ist, dass $\mathrm{X}$ in Wirklichkeit nicht der Fall ist, kann er/sie nun die mentalen Haltungen sowohl des Sprechers als auch des Adressaten entschlüsseln. Oder anders formuliert: A weiß, dass $\mathrm{X}$ nicht der Fall ist und möchte, dass B glaubt, X sei der Fall, während B nicht weiß, dass X nicht der Fall ist und glaubt, dass X der Fall ist, weil er überzeugt ist, dass A intendiert, einen wahren Sachverhalt kommunizieren.

Im Kontrast dazu stehen ironische Äußerungen, die trotz eines entgegengesetzten Wortsinns darauf abzielen von einem Adressaten ,enttarnt' zu werden. Hier entsteht neben der wörtlichen Ebene der Aussage eine zweite - zur ersten im Widerspruch stehende - Sinnebene. Nur wenn der Adressat die Intention des Sprechers in einem ersten Schritt korrekt zugeordnet hat, kann er daran anschließend diese Sinnebene entschlüsseln und der Kommunikationsakt zwischen den beiden Parteien gelingt ohne Missverständnisse. A sagt X, möchte aber einen Sachverhalt Y kommunizieren und möchte ebenfalls, dass B versteht, dass Y der Fall ist. Dabei ist B bewusst, dass A Y kommunizieren möchte und obwohl X kommuniziert wird, 
versteht er Y. Die Anzahl der kursiv dargestellten Elemente der hier konstruierten Formeln illustrieren die Komplexität der Verkettung mentaler Haltungen, die im Fall ironischer Äußerungen dechiffriert werden müssen, um sie korrekt verstehen zu können.

\section{Wünsche und Bedürfnisse}

Neben Intentionen und allgemeinen Handlungszielen sind auch Wünsche (desire) zentrale Faktoren menschlicher Handlungsmotivation. Neben Intentionen und Überzeugungen bilden sie außerdem eine wichtige Kategorie mentaler Haltungen, die Menschen verstehen und attribuieren können. Im Rahmen sozialer Interaktionen ist dabei im Besonderen die Unterscheidung der eigenen salienten Wünsche von denen anderer zentral - eine Leistung, die Kinder ab einem Alter von ca. 18 Monaten meistern. Um diese Fähigkeiten zu testen, entwickelten die beiden Forscherinnen Alison Gopnik und Betty Repacholi (1997) ein Paradigma, welches sich unterschiedliche (im Hinblick auf Kinder jedoch leicht vorhersehbare) Vorlieben in Bezug auf Nahrungsmittel zu Nutze macht. Die Aufgabe der Kinder innerhalb der Experimentalsituation bestand darin, dem Versuchsleiter etwas zu essen zu reichen, nachdem dieser seinen Appetit bekundet hatte. Sie hatten dabei die Wahl zwischen einem für sie tendenziell unattraktiven und einem attraktiven Lebensmittel (Brokkoli/Keks). Kinder im Alter von 14 Monaten überreichten überzufällig häufig das für sie selbst attraktivere Lebensmittel, auch wenn der Versuchsleiter zuvor seine eigene Präferenz für das unattraktivere Lebensmittel deutlich gemacht, beziehungsweise sogar eine negative, ablehnende Reaktion auf das attraktivere Lebensmittel gezeigt hatte. Die Forscherinnen folgerten aus dieser Beobachtung, dass die Kinder noch nicht in der Lage seien, die Wünsche des Versuchsleiters von ihren eigenen zu differenzieren. Eine zweite Stichprobe mit Kindern im Alter von 18 Monaten zeigte dieses Verhalten nicht mehr, so dass davon ausgegangen werden kann, dass Kinder von nun an zwischen eigenen und fremden Wünschen unterscheiden konnten. Im weiteren Verlauf der Entwicklung bis in das Erwachsenenalter hinein sind nun immer weniger explizite Hinweise nötig, um innerhalb sozialer Interaktionssituationen verständlich zu machen, dass bestimmten Handlungen und Reaktionen auf Wünschen und Bedürfnissen beruhen, die sich von den eigenen unterscheiden.

\section{Überzeugungen}

Eine weitere Kategorie mentaler Haltungen, die dem menschlichen Handeln zugrunde liegen kann und daher bei der Handlungsinterpretation berücksichtigt werden muss sind Überzeugungen. Überzeugungen besitzen eine andere Funktion als Wünsche und Intentionen und 
nehmen daher auf andere Art und Weise Einfluss auf das menschliche Handeln. Kinder erfassen die Bedeutung von Überzeugungen (belief) für menschliche Handlungen etwa ab einem Alter von dreieinhalb Jahren. Ein wichtiger Fortschritt verglichen mit allen zuvor bereits erlangten Entwicklungsstufen auf der ToM-Skala besteht darin, dass von nun an auch Handlungen, die sich möglicherweise nicht sinnvoll mit den realen beobachtbaren Gegebenheiten einer bestimmten Situation vereinbaren lassen, mit Hilfe der Zuschreibung von (falschen) Überzeugungen erklärbar sind.

Heinz Wimmer und Josef Perner (1983) machten sich diese theoretischen Überlegungen zu Nutze, als sie die sogenannte False-Belief-Aufgabe entwickelten, um das In-Erscheinungtreten dieser kognitiven Fähigkeiten im Kindesalter nachzuvollziehen. Sie konfrontierten Kinder in einem Alter von drei bis vier Jahren mit einer kurzen Bildergeschichte, die folgendermaßen wiedergegeben werden kann: Ein Junge (Maxi) versteckt eine Tafel Schokolade in einem roten Schrank und geht nach draußen. In seiner Abwesenheit räumt seine Mutter das Zimmer auf und legt die Schokolade in einen blauen Schrank. Maxi kommt kurz darauf zurück und ist hungrig. Die erste, vom Versuchsleiter an die Kinder gestellte Frage lautet nun, wo Maxi die Schokolade suchen wird. Außerdem überprüfen die Forscher das korrekte Situationsverständnis der Kinder, indem sie verifizieren, ob die Kinder wissen, wo sich die Schokolade tatsächlich befindet. Entscheiden sich die Kinder im Hinblick auf die erste Frage für den roten Schrank und können sie auch die tatsächliche Position des Gegenstandes korrekt benennen, so die Schlussfolgerung der Entwicklungspsychologen, dann müssen sie folglich in der Lage sein, Maxi eine falsche Überzeugung zuzuschreiben. Bis heute wurden zahlreiche Variationen dieser Testaufgabe entwickelt, die die Zuschreibung von falschen Überzeugungen nicht mehr nur wie im Fall der Maxi-Geschichte im Hinblick auf einen unerwarteten Ortswechsel, sondern auch z. B. in Bezug auf einen unerwarteten Inhalt erforderlich machen (Wellman, Cross \& Watson, 2001).

\section{Verkettungen mentaler Haltungen}

Die Komplexität solcher Aufgabenstellungen lässt sich variieren, um die Leistungsfähigkeit der ToM zu veranschaulichen. So entstehen in einem sozialen Kontext häufig Situationen, die ein Verständnis bzw. die ,Entzifferung' von z. T. vielfachen Verkettungen voneinander abhängiger mentalen Haltungen erfordert. Die hohe kognitive Leistung, die erforderlich ist, um solche Überzeugungen oder Intentionen höherer Ordnung (X denkt, dass Y denkt, dass A der Fall ist) richtig zu interpretieren, spiegelt sich in der Entwicklungschronologie der 
ToM-Skala wider. Erst ab einem Alter von ca. sechs Jahren sind Kinder in der Lage, einfache verschachtelte Zusammenhänge (second-oder mental state attribution) richtig zu interpretieren. Gesunde Erwachsene lösen in der Regel Verkettungen bis zur vierten Ordnung mühelos. Ab Verkettungen mit fünf Stufen (A denkt, B sei der Überzeugung, dass C weiß, dass D denkt, dass E meint, X sei der Fall) erhöht sich die Fehlerrate beim Rückentschlüsseln der Intentionen oder Überzeugungen auch bei gesunden Erwachsenen jedoch massiv (Dunbar, 2000, S. 241). Es stellt sich mit einem Blick auf diese Ergebnisse die Frage, mit Hilfe von welchen kognitiven Ressourcen Menschen solche komplexen Aufgaben lösen bzw. aufgrund welcher kognitiver Einschränkungen die Fehlerrate ab einem bestimmten Komplexitätsgrad steigt. Bevor diese Frage beantwortet werden kann, gilt es jedoch weitere kognitive Leistungen vorzustellen, die in der Vergangenheit mit dem ToM-Konzept in einen Zusammenhang gestellt wurden. Eine wichtige Aufgabe, die neben den False-Belief-Aufgaben zur Messung von ToM-Kompetenzen genutzt wird sind sogenannte Appearance-Reality-Aufgaben (z. B. Carlson, Moses \& Claxton, 2004).

\section{Das Appearance-Reality-Problem}

In unserer sozialen Umgebung sind wir häufig mit der Aufgabe konfrontiert, zwischen ,Schein und Sein' (, appearance and reality', nachfolgend , $\mathrm{AR}^{\star}$ ) zu unterscheiden. Gesunde Erwachsene erbringen vergleichbare kognitive Leistungen in zahlreichen unterschiedlichen Situationen. Sie sind z. B. in der Lage, Sinnestäuschungen (z. B. Trugbilder), eigene und fremde situationsbezogene Missverständnisse, falsche Überzeugungen und mutwillige Täuschungen während der Interpretation verschiedener Situationen zu berücksichtigen.

Um herauszufinden, welche konkreten kognitiven Prozesse und Leistungen diesen sichtbaren Kompetenzen zugrunde liegen, untersuchten Flavell und seine Kollegen (z. B. 1982, 1993) die Entwicklung dieser Fähigkeit im Kindesalter im Rahmen mehrerer Studien, in denen sie unterschiedliche Aufgaben zur AR-Unterscheidung stellten. Sie zeigten ihren Testteilnehmern z. B. einen Gegenstand, der einem Stein zum Verwechseln ähnlich sieht, in Wirklichkeit jedoch ein Schwamm ist, dessen wahre Identität nicht ersichtlich, aber zu erfühlen ist (Flavell, 1986, S. 419). In einer vergleichbaren Testaufgabe zeigten sie einen weiBen Gegenstand, der - wenn er hinter einen Farbfilter gehalten wird - scheinbar seine Farbe wechselt (ebd.). Es zeigte sich, dass Kinder unter drei Jahren Schwierigkeiten haben, zwischen der realen und scheinbaren Erscheinungsform von Gegenständen zu unterscheiden. Erst im Alter zwischen sechs und sieben Jahren lösen sie die gestellten Aufgaben zuverlässig. 
Anstatt physischer Gegenstände können in diesem Sinne auch innere Vorgänge (etwa die Unterscheidung echter und vorgetäuschter Emotionen) im Fokus der Aufmerksamkeit stehen (z. B. Harris, Donnelly, Guz \& Pitt-Watson, 1986). So ist es denkbar, dass die sichtbare Mimik oder Gestik einer Person nicht mit ihrer tatsächlichen emotionalen Haltung übereinstimmt - ein Mensch nach dem Auspacken eines Geschenkes bspw. Freude und Dankbarkeit zeigt, in Wirklichkeit jedoch Enttäuschung empfindet. Diese Fähigkeit lässt sich überprüfen, indem Kindern z. B. eine kurze Geschichte präsentiert wird. In dieser Geschichte wird eine Situation konstruiert, in welcher es für einen Charakter (z. B. Maxi) angemessen ist, entweder positiv oder negativ zu empfinden. Eine solche Geschichte könnte folgendermaßen lauten: Maxi spielt mit seinen Freunden. Einer seiner Freunde erzählt einen gemeinen Witz über Maxi und alle außer Maxi finden ihn sehr komisch und lachen. Maxi möchte nicht, dass die anderen sehen, wie er sich fühlt und dass sie ihn einen Spaßverderber nennen. Daher versucht er, seine Gefühle zu verbergen. Nach einigen anschließenden Gedächtnisfragen zu dem allgemeinen Verständnis der Geschichte müssen die Testteilnehmer nun entweder verbal oder nonverbal (z. B. mit Hilfe von Karten, auf denen abstrakte Gesichter mit unterschiedlichen typischen emotionsanzeigender Mimik zu sehen sind) beantworten, wie Maxis Befinden nach außen wirkt und wie er sich tatsächlich fühlt. Zusätzlich wurden die Kinder in einigen Testdesigns aufgefordert, ihre Antworten zu begründen (ebd.). Der Alterseffekt (d. h. erst Kinder ab einer gewissen Altersgrenze beantworten die gestellten Fragen überzufällig häufig mit der korrekten Antwort), der von Harris und seinen Kollegen mit Hilfe dieses Versuchsdesigns ermittelt wurde, ließ sich in vergleichbaren Studien zuverlässig reproduzieren. Friend und Davis argumentieren, dass diese Ergebnisse für einen kognitiven Entwicklungssprung der Fähigkeit, Objekte und Situationen repräsentieren zu können, sprechen (Friend \& Davis, 1993, S. 912). Objekte können nicht gleichzeitig weiß und blau bzw. gleichzeitig ein Stein und ein Schwamm sein. Menschen sind in der Regel - bezogen auf eine bestimmte Situation - nicht gleichzeitig traurig und glücklich. Flavell (1986, S. 422) schreibt: „As adults, we easily resolve the seeming contradiction by identifying one representation of its property or identity with its present appearance and the other with its reality.“ Gesunde Erwachsene können jedoch nicht nur den Unterschied zwischen dem Erscheinungsbild und den tatsächlichen Eigenschaften einer Situation erkennen, sie können diese Situation auch abstrakt und bewusst reflektieren. So erkennen sie z. B. unterschiedliche mögliche Relationen zwischen Schein und Sein eines Objektes an, dessen Erscheinungsbild mit seiner Identität sowohl übereinstimmen als auch von ihr abweichen kann. Erwachsene leisten dies im Hinblick auf unterschiedliche Typen von AR-Aufgaben und subtile Abstufungen im Grad 
der Abweichung zwischen Schein und Sein. Wir unterscheiden also ohne Schwierigkeiten z. B. zwischen dem Konzept eines Originals, realistisch erscheinenden ,guten`Fälschungen, nicht-realistisch erscheinenden ,schlechten“ Fälschungen und sogar unecht erscheinenden Originalen. Um dies zu leisten, müssen wir dazu in der Lage sein, von ein und demselben Objekt oder ein und derselben Situation zwei verschiedene kognitive Repräsentationen zu erstellen, wobei sich eine davon auf die Erscheinung (appearance) und eine auf die tatsächliche Identität (reality) des Objektes oder der Situation bezieht. Eine Person kann also gleichzeitig registrieren, dass ein Objekt eine bestimmte Erscheinungsform hat, und gleichzeitig wissen, dass seine Identität eine andere ist. Falsche Überzeugungen zu dekodieren und Schein und Sein eines Objektes richtig zu repräsentieren erfordert damit - strukturell gesehen - eine vergleichbare Kognitionsleistung. Diese Kognitionsleistung untersuchten Frye, Zelazo \& Palfai (1995) mit Hilfe von Kartensortierungstests, bei denen Kinder dieselben Karten nach unterschiedlichen Dimensionen (Regeln), z. B. nach Farbe und Motiv sortieren müssen. Je mehr Regeln Kinder gleichzeitig beachten mussten, um die Aufgabe zu lösen, desto schwieriger fiel es ihnen, die Aufgabe zu lösen. Insbesondere die Leistung, nach einer Dimension (Farbe) zu sortieren und eine andere Dimension (Motiv) zu ignorieren fiel den Kindern schwer. Allerdings korrelierten die Ergebnisse dieses Tests mit den Fähigkeiten der Kinder, falsche Überzeugungen zu verstehen, besonders stark. Dieser Befund untermauert den Verdacht, dass hier ein gemeinsamer kognitiver Mechanismus zugrunde liegt, den Frye und seine Kollegen ,rule-based reasoning' nennen. (ebd., S. 522 f.). Dieser Mechanismus hilft dabei, eine Dimension der Karten zu ignorieren, wenn es darum geht, sie nach einer anderen Dimension zu sortieren. Er hilft auch dabei zu erkennen, dass ein Gegenstand eine bestimmte Erscheinung hat, tatsächlich aber etwas anderes ist. Die Fähigkeit nach unterschiedlichen Indizes eine bestimmte Situation auf unterschiedliche Art und Weise zu bewerten hilft gesunden Erwachsenen ebenfalls dabei zu verstehen, dass ein und dieselbe Person unterschiedliche Repräsentationen desselben Gegenstandes bilden kann. Sie gestattet dabei auch zu verstehen, dass unterschiedliche Personen unterschiedliche oder vergleichbare Repräsentationen von ein und demselben Gegenstand bilden können usw. (Flavell, 1986, S. 418).

\section{Die Rekonstruktion fremder und eigener Perspektiven}

Gesunde Erwachsene sind in der Lage, sich ,in die Schuhe“ anderer zu versetzen. Damit ist gemeint, dass sie neben der eigenen auch fremde Perspektiven kognitiv repräsentieren können. Moll und Meltzoff (2011, S. 286) beschreiben diese Fähigkeit mit folgenden Worten: 
Perspectivity in its mature, adult form even goes beyond the ability to determine a specific person's point of view at a certain moment in time. It entails the general comprehension that one and the same thing or event can be viewed or construed differently depending on one's standpoint - whether this is a visuo-spatial, epistemic, conceptual, or affective standpoint.

,Perspectivitiy‘ bezieht sich also nicht ausschließlich auf wahrnehmungsbezogene Perspektivübernahmen, die die räumlichen Aspekte einer bestimmten Situation betreffen (,visual perspective-taking ${ }^{6}$ ). Es sind ebenfalls erfahrungsbezogene Perspektivübernahmen (,experiential perspective-taking ${ }^{\circ}$ gemeint, wobei Situationen mit Bezug zu dem spezifischen Wissenszugang und der Überzeugungshaltung einer Person rekonstruiert werden müssen. Kindern fällt es bis zu einem bestimmten Alter schwer, die Perspektive anderer nachzuvollziehen (Newcombe \& Huttenlocher, 1992). Diese Fähigkeit entwickelt sich vielmehr über eine Zeitspanne von mehreren Jahren hinweg im Vorschul- und Schulalter. Bereits in ihrem ersten Lebensjahr beginnen Kleinkinder ihren eigenen Aufmerksamkeitsfokus mit demjenigen ihrer Bezugsperson ,zu koordinieren“. Dies ist dann zu beobachten, wenn Kinder beginnen, auf Gegenstände zu zeigen (pointing) bzw. den Blicken ihrer Interaktionspartner zu folgen (gaze following). Die zuvor ausschließlich dyadische Interaktion zwischen Kindern und ihren Bezugspersonen ist nun insofern als triadisch zu charakterisieren, als neben den beiden Interaktionspartnern ,dritte“ Gegenstände der unmittelbaren Umwelt in die Kommunikation eingebunden werden. Dieser Vorgang wird in der entwicklungspsychologischen Literatur mit dem Begriff, joint attention“ (nachfolgend ,JA') oder , shared attention“ bezeichnet. Der gemeinsame Aufmerksamkeitsfokus eines Kindes und seiner Bezugsperson kann sich auf physische Gegenstände aus dem gegenwärtigen Umfeld, andere Menschen, später in der Entwicklung aber auch auf innere Vorgänge (z. B. die Gedanken, Emotionen, Überzeugungen anderer) richten. Das besondere an der kognitiven Leistung, die mit diesem Verhalten einhergeht, ist, dass neben dem jeweiligen Objekt, dem die Aufmerksamkeit gilt, gleichzeitig die Haltung der anderen beteiligten Personen gegenüber diesem Objekt mitverfolgt und bewertet wird (Tomasello, 1995, S. 105). Kinder fokussieren im Zuge einer joint attentionInteraktion also nicht lediglich dasselbe Objekt wie der Interaktionspartner, sie registrieren vielmehr gleichzeitig den Aufmerksamkeitsfokus ihrer Bezugsperson und das Objekt im Fokus der Aufmerksamkeit. Es ist umstritten, bis zu welchem Grad diese Koordination bewusst stattfindet oder lediglich behavioristischen Verhaltensimpulsen folgt. Doch auch wenn Menschen diesen Vorgang im Zuge des JA nicht zwingend bewusst reflektieren, so werden sie 
doch kognitiv ,registriert' und bei der Informationsverarbeitung berücksichtigt (Hobson, 2005, S. 201).

Die Koordination gemeinsamer Wahrnehmung zwischen zwei Individuen hinsichtlich von Objekten (oder anderen Individuen) der realen Welt dient in der frühen Kindheit zunächst einmal kommunikativen Zwecken und erleichtert das kindliche Lernverhalten. Der gemeinsame Fokus auf ein Referenzobjekt und die Fähigkeit, gleichzeitig z. B. die emotionale Haltung einer Bezugsperson diesem Objekt gegenüber zu registrieren, erleichtert die Orientierung in einer komplexen, z. T. noch unbekannten Welt maßgebend. So ist es Bezugspersonen mit verbalen und nonverbalen Mitteln schon sehr früh möglich, ihren Kindern den Unterschied zwischen gefährlichen und ungefährlichen Gegenständen zu signalisieren. Umgekehrt können Kinder, wenn sie mit neuen Gegenständen und Situationen konfrontiert sind, im Fall von Unsicherheit Informationen von ihren Bezugspersonen einfordern und sich daran orientieren. Sie erhalten auf diesem Weg eine Richtlinie im Umgang mit dem Unbekannten. Ähnlich verhält es sich, wenn Kinder neue Vokabeln erlernen. Ohne die Fähigkeit zur Koordination von Wahrnehmung und Aufmerksamkeit müssten Eltern die Äußerung eines Wortes mit dem zufälligen Fokussieren des Kindes auf das entsprechende Objekt abstimmen, damit eine entsprechende Verknüpfung von Label und Objekt stattfinden könnte. Auf der anderen Seite könnten die Kinder fälschlicherweise Bezeichnungen mit einem Objekt verknüpfen, die zufällig genau dann durch das Elternteil benannt werden, wenn ein bestimmtes Objekt im Zentrum der kindlichen Aufmerksamkeit steht (Sabbagh \& Baldwin, 2005). Dies gilt nicht nur für das Erlernen einfacher Bezeichnungen, sondern auch für die weiterführende Charakterisierung eines Gegenstandes. Nachdem ein Objekt im kindlichen Wortschatz ein einfaches Label (z. B. ,Ball') erhalten hat, können darauf aufbauend bestimmte Qualitäten desselben bezeichnet und Relationen zwischen Objekten hergestellt werden. Hierbei müssen Kinder herausfiltern können, welche Kategorie von Eigenschaften (z. B. Textur, Farbe oder Form) jeweils im Fokus der Aufmerksamkeit steht. Dies erlaubt die flexible Übertragung und Verknüpfung von verschieden Bezeichnungen und Bedeutungen auf Objekte jenseits des ursprünglichen Kontextes, in welchem das neue Wort erlernt wurde.

Um dies zu gewährleisten, ist ein grundlegendes Verständnis von Intentionalität erforderlich. Wenn bereits junge Kinder auf ein Objekt zeigen, setzt dies voraus, dass die beobachtende Person versteht, dass es sich nicht nur um eine zufällige Bewegung der Hand handelt - mit dem Fingerzeig also etwas ,gemeint“ ist. Das Kind nutzt eine entsprechende Geste auf der anderen Seite erst dann, wenn es versteht, dass sie von seinem Gegenüber als intentional 
wahrgenommen wird. Synchron mit der Entwicklung eines grundlegenden Verständnisses für Intentionalität ist die Fähigkeit, JA zu initiieren und den Aufmerksamkeitsfokus der Bezugsperson z. B. mit einem Fingerzeig zu manipulieren, ab einem Alter von 9 Monaten zu beobachten (Desrochers, Morrisette \& Ricard, 1995, S. 96). Die Subtilität und Komplexität der Initiations- und Beteiligungstechniken im Hinblick auf die Vorgänge des JA nimmt anschließend mit zunehmendem Alter zu. Mit den Worten von Corkum und Moore (1995, S. 81):

[...] once the infant is reliably engaging in joint attention, the experiences offered by this new triadic interactive form of behavior will afford progress in the infant's conceptualization of attention and set the child on the road to the discovery of all forms of social life that rests on sharing attention with others.

Etwa in einem Alter von 18 Monaten zeigen Kinder, dass sie die Selektivität der Wahrnehmung ihres Gegenübers erkennen. Sie erkennen dann z. B., welche für sie selbst sichtbaren Objekte für eine andere Person ggf. nicht zu sehen sind. Etwa ab einem Alter von drei Jahren verstehen Kinder die räumlichen Unterschiede und dazu gehörigen deiktischen Relationen zwischen eigenen und fremden Bezugsstandpunkten und Objekten in der Umwelt. Sie verstehen also, dass ein Objekt, dass sich ,links von“ oder ,vor ${ }^{6}$ ihnen selbst befindet, sich gleichzeitig ,rechts von“ oder ,hinter' jemandem anders befinden kann (Perner, Brandl \& Garnham, 2003). In einem Alter von 4,5 Jahren beginnen Kinder zu verstehen, dass Objekte, die sich aus dem eigenen Blickwinkel in einer bestimmten Art und Weise darstellen, von einem anderen Standpunkt aus anders aussehen können (Perner, Slummer, Sprung \& Doherty, 2002). Im Erwachsenenalter sind wir dann dazu in der Lage, unseren eigenen sowie einen fremden kognitiven Bezug zur Umwelt, die durchaus miteinander konkurrieren können, kognitiv zu repräsentieren und die Handlungen und Wahrnehmung anderer darauf bezogen zu interpretieren. Wir können also 1. unsere Aufmerksamkeit mit der Wahrnehmung eines anderen koordinieren. Wir können 2. berücksichtigen, dass sich Objekte und Situationen aus der fremden Perspektive evtl. anderes darstellen als aus unserer eigenen. Wir können 3. die inneren Haltungen anderer Objekten und Situationen gegenüber, die ggf. von unseren eigenen abweichen, nachvollziehen. Perner argumentiert, dass ein solcher Mechanismus der kognitiven Perspektivübernahme vielen sozialen Fähigkeiten zugrunde liegt, die mit der ToM assoziiert werden (Perner et al., 2003). Diese Fähigkeiten umfassen z. B. das Verstehen falscher Überzeugungen (epistemic perspective taking), das Erkennen eines falschen Er- 
scheinungsbildes (z. B. wenn ein Gegenstand wie ein Apfel aussieht, es sich jedoch tatsächlich um eine Kerze handelt) (Flavell, Flavell \& Green, 1983) (conceptual perspective taking) und die Fähigkeit metalinguistische Verbindungen (Doherty \& Perner, 1998) zu ziehen (ein und dasselbe Objekt kann mit unterschiedlichen Bezeichnungen belegt werden z. B. ,rabbit‘ oder , bunny'). Damit ist eine mögliche Erklärung dafür gegeben, aus welchem Grund sich diese Fähigkeiten synchron zueinander im Vorschulalter entwickeln. Die Überschneidung des Entwicklungszeitraumes ist nicht dem Zufall geschuldet. Sie lässt sich damit erklären, dass hier für zwei verschiedene soziale Fähigkeiten ein bestimmter kognitiver Mechanismus relevant ist. Dieser Mechanismus ermöglicht es uns, ein und dasselbe Objekt oder Ereignis oder eine bestimmte Situation in unterschiedlicher Art und Weise in Abhängigkeit von unterschiedlichen Perspektiven zu konzeptualisieren bzw. zu interpretieren (Moll \& Meltzoff, 2011, S. 297).

\section{Fremde und eigene Imagination}

Gesunde Erwachsene unterscheiden nicht nur zwischen den unterschiedlichen Perspektiven verschiedener Menschen bzw. der scheinbaren und echten Identität eines Gegenstandes. Sie unterscheiden auch - in der Regel ohne Mühe - zwischen realen und imaginativen Gegenständen und Situationen. Diese Fähigkeit manifestiert sich erstmals im sogenannten kindlichen Fiktionsspiel (nachfolgend ,FS '). ${ }^{11}$ Um uns an einer solchen Spielepisode beteiligen zu können, müssen wir in der Lage sein, Gegenständen und Situationen fiktive Eigenschaften zuschreiben und nachzuvollziehen, dass unsere Spielpartner ggf. dasselbe tun. Diese fiktiven Eigenschaften unterscheiden sich in der Regel von den realen - und damit unmittelbar wahrnehmbaren Eigenschaften - und müssen daher kognitiv attribuiert werden. Gleichzeitig ist es entscheidend, zu wissen, wann ein Spielpartner welche fiktiven oder realen Eigenschaften attribuiert, um im Rahmen des FS miteinander interagieren zu können. Im Rahmen des FS - so scheint es - sind also kognitive Leistungen (z. B. die Attribution bestimmter mentaler

\footnotetext{
${ }^{11}$ Im englischen Forschungsraum wird die kindliche Spielform, die in diesem Abschnitt im Fokus der Aufmerksamkeit steht, kontextabhängig als ,pretend play', ,make-believe`, ,fantasy play', ,symbolic play oder , acting-as-if ${ }^{\star}$ bezeichnet. Josef Perner weist darauf hin, dass mit der unterschiedlichen Begriffswahl unterschiedliche Schwerpunkte in der konzeptuellen Beschreibung derjenigen Kognitionsprozesse einhergehen, die dieser Spielform zugrunde liegen. So wird mit der Bezeichnung als, fantasy play ${ }^{\circ}$ die Trennung zwischen Phantasie und Realität besonders betont, im Fall des ,make-believe die Intention hervorgehoben, ,jemanden etwas Glauben machen' zu wollen und mit ,symbolic play' auf den kognitiv-repräsentativen Charakter derjenigen Kognitionsprozesse hingewiesen, die diese Fähigkeiten ermöglichen. Aufgrund der vielfältigen Erscheinungsformen der Spielform ist es schwierig zu entscheiden, welcher dieser Begriffe die relevanten Kognitionsprozesse am besten erfasst. Im Folgenden sollen alle Formen des kindlichen Spiels, die in irgendeiner Form mit dem Kognitionsakt der Imagination in Verbindung stehen, im Sinne der begrifflichen Klarheit als ,Fiktionsspiel' (FS) bezeichnet werden
} 
Haltungen und Vorgänge) zu erbringen, die für die ToM zentral sind. Lillard (1993, S. 348) schreibt:

Pretend play has $[\ldots]$ been of great interest to researchers studying children's understanding of the mind. One reason for this interest is that pretense seems to require many of the same skills as mental state understanding, and these skills [the ability to represent one object as two things at once, the ability to see one object representing another, and the ability to represent mental representations] seem to emerge precociously in pretense.

Der Zusammenhang zwischen ToM und FS ist mehr als nur intuitiv. Autistische Kinder zeigen spezifische Defizite in beiden konkreten Bereichen kognitiver Entwicklung, also dem FS und den spezifischen Kompetenzen auf der ToM-Aufgabenskala (Youngblade \& Dunn, 1995). Kinder, die an frühkindlichem Autismus leiden, haben nicht nur Schwierigkeiten beim Lösen von false-belief Aufgaben ${ }^{12}$ (einer der zentralen Aufgaben der entwicklungschronologischen ToM-Skala), sondern sind auch nur in gehemmter Form oder gar nicht beim Fiktionsspiel zu beobachten bzw. lassen sich nur schwer dazu animieren (Charman \& Baron-Cohen, 1997). Es stellt sich die Frage, welche kognitiven Mechanismen dem FS und der ToM gleichermaßen zugrunde liegen.

Das im Kindesalter zu beobachtende FS kann unterschiedlich komplexe Formen annehmen. Im Verlauf der Entwicklungschronologie nimmt mit höherem Alter auch der Grad der Abstraktionen innerhalb der einzelnen Spielepisoden zunächst einmal zu, bevor dieses Spielverhalten ab dem Jugendalter nicht mehr offen bzw. interaktiv gezeigt wird. Gesunde Kinder beschäftigen sich mit fiktiven Gegenständen bzw. schreiben realen Gegenständen fiktive Eigenschaften ab einem Alter von etwa 18 Monaten zu. Sie beginnen zu diesem Zeitpunkt damit, Gegenstände zu ,entfremden` und ihnen eine neue Identität zuzuweisen. So wird aus einem Waschlappen ein Handtuch, aus einem Bauklotz ein Kamm und aus einer einfachen leeren Müslischale eine Teetasse für die Lieblingspuppe. Diese scheinbar simple Form des Spielens erfordert die Fähigkeit, die realen physikalischen Eigenschaften eines Objektes mental gegen die Attribute eines imaginierten Gegenstandes auszutauschen. Die Flexibilität der Kinder reicht dabei soweit, dass die vorhandenen Requisiten durch rein mentale Elemente ergänzt werden können - imaginärer Tee z. B. in die tatsächlich leere Teetasse ,gegossen“ wird. Dabei muss nicht zwingend ein Substitut wie beispielsweise Wasser in der Tasse vorhanden sein, sie kann als leeres Gefäß ohne Probleme mit allen Eigenschaften und

\footnotetext{
${ }^{12}$ Dieses zentrale Versuchsparadigma der kognitiven Entwicklungspsychologie wurde unter Punkt 2.2 Einzelne Dimensionen der Handlungsmotivierung (Überzeugungen) näher beschrieben.
} 
Funktionen einer gefüllten Teetasse ausgestattet und in das Spiel integriert werden. Später entwickeln sich anspruchsvollere Varianten des Fiktionsspiels, die nicht nur Requisiten, sondern auch die Interaktion mit einem oder mehreren Spielgefährten beinhalten. Harris und seine Kollegen (1993, S. 2) weisen darauf hin, dass Kinder, um sich erfolgreich an einem solchen interaktiven Fiktionsspiel beteiligen zu können, in der Lage sein müssen, die Handlungen und Äußerungen ihrer jeweiligen Spielkameraden in einem übertragenen Sinne zu verstehen und eine Welt zu konstruieren, die nicht ihrem realen Umfeld entspricht:

From the end of their second year, children can keep track of pretense stipulations and scenarios and implicitly distinguish them from real states of affairs [...]. Roughly one and a half years later, in their fourth year, children then acquire the ability to explicitly distinguish and contrast pretense and real states of affairs, and pretense stipulations of different people towards the same real situation. (Rakoczy, 2004, S. 14).

Racoczy schließt dies u. a. aus der Beobachtung, dass die Realisierung solcher spielerischen fiktiven Szenarien in der Regel kommentarlos vonstattengeht. Ein Heraustreten aus der fiktionalen Welt ist für eine gelingende und stimmige Kommunikation unter den Spielteilnehmern nicht notwendig. Das bedeutet, dass Kinder eine fiktionale Welt und ihre Regeln nicht nur in ihrem eigenen Geist abbilden, sondern ein ähnliches mentales Vorstellungsvermögen zusammen mit den jeweils aktuellen Repräsentationen imaginärer Objekte und Verhältnismäßigkeiten ihren Spielpartnern zuschreiben und für die Dauer des Spiels aufrechterhalten müssen. Nur so können sie die unkommentierten Handlungen, die sich auf imaginäre Gegenstände beziehen, korrekt dekodieren und adäquat auf die Geschehnisse im Sinne der gerade relevanten Fiktionswelt reagieren.

Kinder kreieren außerdem häufig vollständig imaginäre Gegenstände oder sogar Personen ein gutes Beispiel hierfür ist der berühmte ,imaginäre Freund‘. Hierfür müssen sie in der Lage sein, kognitive Repräsentationen von Objekten und Personen zu entwerfen, deren Eigenschaften durch keine sicht- oder fühlbaren physikalischen Attribute eines vorhandenen Objekts gestützt werden. Dennoch sind diese imaginären Objekte und Personen häufig mit stabilen und komplexen Charaktereigenschaften und (imaginären) physischen Attributen ausgestattet. In der interaktiven Form des Fiktionsspiels sind beide bereits beschriebenen Ausprägungen des Spiels häufig miteinander kombiniert. Wenn Kinder ab einem Alter von knapp zwei Jahren ihr FS mit einem Elternteil oder auch gleichaltrigen Spielpartnern koordinieren, beginnt eine solche typische Episode zumeist mit einem Set einfacher Vereinbarungen, die zu Beginn von den teilnehmenden Spielpartnern festgelegt werden. Dabei reicht 
eine einzige explizite Äußerung eines der teilnehmenden Spielpartner (z. B. ,Du bist die Mutter, ich bin das Kind“) oft aus, um das Spiel zu initiieren. Eine der interessantesten Eigenschaften dieser Festlegungen besteht in ihrer, Generativität‘. Sind sie einmal aufgestellt, aktivieren sie eine Reihe möglicher folgerichtiger Gesetzmäßigkeiten und können im Zuge des weiteren Spiels die Einbindung verschiedener neuer Requisiten oder Regeln ohne weitere Absprachen autorisieren bzw. ausschließen. Dies geschieht hauptsächlich über Schlussfolgerungen, die von dem zu Beginn des Spiels erstellten Regelkatalog zusammen mit den aus dem realen Kontext bekannten Gesetzmäßigkeiten ausgehen. Bereits Zweijährige sind in dieser Hinsicht in der Lage, die ihnen aus der echten Welt bekannten physikalischen Gesetze in das Spiel einzubinden. In diesem Sinne gilt beispielsweise: Wird eine Schachtel als Badewanne angenommen, die mit imaginärem Wasser gefüllt ist, so sind alle Gegenstände, die sich in dieser Box befinden oder mit ihrem Inhalt in Berührung kommen, folgerichtig im Rahmen des Fiktionskontextes nass (Harris, 2000, S. 13). Auch mentale Schemata und Skripte dienen in diesem Zusammenhang zum Verständnis der innerhalb des Spiels auftretenden nicht abgesprochenen Handlungen. Der Entschluss, Baumstümpfe als Bären zu behandeln, rechtfertigt, einen in der Nähe befindlichen Ast als Schwert zur Verteidigung aufzunehmen. Dies verstehen Kinder ohne weitere Erläuterungen der Agierenden, da die Regeln der Fiktionswelt diejenigen der realen Welt für die Dauer des Spiels scheinbar überlagern und für das Verständnis der Geschehnisse eine ausreichende Grundlage bieten. Aus einigen wenigen vereinbarten Bedingungen entfaltet sich auf diese Weise eine komplexe Spielwelt. Bleiben die gezeigten Handlungen in diesem Sinne folgerichtig und im Einklang mit der initialen Abmachung, bedürfen sie keiner weiteren Absprache zwischen den Spielteilnehmern. Damit bleiben die Grenzen der Fiktionswelt und die Spielerfahrung unverletzt (ebd., S. 12).

Neben dieser Tendenz zur Kontinuität besitzen Kinder außerdem eine beachtliche generative Adaptivität im Hinblick auf die jeweils gemeinschaftlich erschaffenen imaginativen Welten. Sie können ohne Probleme eine Regel, die für eine konkrete Situation gilt, in einem neuen Kontext anders interpretieren. Auch die Wahl der für das Spiel zu Hilfe genommenen Gegenstände zeugt von einer hohen kognitiven Wendigkeit. So kann der im letzten Spiel als Schwert interpretierte Ast in einer zeitlich nicht weit entfernten Situation als Paddel eines Ruderbootes instrumentalisiert werden, ohne dass es zu kognitiven Konflikten kommt:

Children's flexibility also highlighted the fact that it was not the prop per se that was cueing them about what to do. They did not perceive anything intrinsically sandwich-like or soap-like about the brick. Instead, when they 
acted on the prop they were guided by the currently valid stipulation. So, although the brick obviously retained the same objective features across the two different episodes, the children pretended that it had different features depending on the episode in which it was embedded. (Ebd., S. 13)

Die involvierten Gegenstände wie der Baumstumpf oder die Schachtel erhalten innerhalb einer Fiktionswelt also eine neue Bezeichnung oder einen neuen Namen, von denen die neuen fiktiven Eigenschaften des Objektes abhängen. Diese ,Labels‘, die über die gesamte Spieldauer kognitiv aufrechterhalten werden, sind erstaunlich durchgreifend. Handlungen und Manipulationen an einem Gegenstand verstehen Kinder konsequent im Sinne der Fiktionswelt und nicht basierend auf den realen Eigenschaften des Gegenstandes. Es lassen sich bei solchen Spielsituationen keine Reaktionszeitverzögerungen beobachten, die eventuell darauf hindeuten würden, dass die reale und fiktionale Identität der betroffenen Gegenstände und Personen während der kognitiven Verarbeitung miteinander konkurrieren. Um dies leisten zu können, ist nicht nur eine Leistung des Imaginierens gefragt, sondern in vielen Fällen auch die für die Dauer der Spielsituation begrenzte Zurückstellung der tatsächlichen und unmittelbar sichtbaren physikalischen Eigenschaften des jeweiligen Objektes (Leslie, 1987, S. 416). Kinder erleiden dabei keinen Realitätsverlust, sondern können zu jedem Zeitpunkt des Spiels, wenn sie danach gefragt werden, einschätzen, welche Gegebenheiten real und welche fiktiv sind (Perner, 1991, S. 68). Es stellt sich die Frage, wie Kinder in der Lage sind kognitive Inferenzen, Überzeugungen und Lernleistungen im Rahmen des FS von solchen im Rahmen realitätsbezogener Interaktionen zu trennen. (Leslie, 1987, S. 416 ff.). Sind sich Kinder bereits in einem so jungen Alter (18 Monate) über die ,Natur' imaginativer Situationen bewusst? Auch wenn ältere Kinder und Erwachsene durchaus in der Lage sind, den kognitiv-repräsentativen Charakter von solchen mentalen Modellen zu erkennen, reicht es Perner zufolge für die Teilnahme an den verschiedenen Formen des Fiktionsspiels aus, unterschiedliche kognitive Modelle der eigenen Umwelt bilden zu können (Perner, 1991, S. 68).

Ob sich Kinder (in einem begrenzten Rahmen) der Natur kognitiv-repräsentativer Einheiten bewusst sind oder nicht, ihnen muss in jedem Fall ein Set kognitiver Ressourcen zur Verfügung stehen, welches ihnen erlaubt, imaginative Vorgänge von (teilweise oder vollständig) realitätsbezogenen Vorgängen zu trennen. Shaun Nichols und Stephen P. Stich erklären diese Fähigkeit damit, dass Kinder neben einem grundlegenden Verständnis handlungsmotivierender Haltungen wie Wünsche und Intentionen nun zusätzlich in der Lage sind, eine 
neue Kategorie mentaler Haltungen zu verstehen. Diese Kategorie benennen die beiden Autoren mit dem Mentalverb ,supposing (Nichols \& Stich, 2003). Sie argumentieren, dass Kinder, die ein Fiktionsspiel unterhalten, bestimmte Dinge als gegeben annehmen, indem sie ihnen gegenüber eine bestimmte Haltung einnehmen. Diese Haltung unterscheidet sich von ihrer Haltung gegenüber realitätsbezogenen Vorgängen. Unter der Voraussetzung, dass alle Spielteilnehmer z. B. ,davon ausgehen', dass ein Baumstumpf imaginativ ein gefährlicher Bär und ein Ast imaginativ ein Schwert ist, wird das Schlagen des Baumstumpfes mit dem Ast im Rahmen des Fiktionsspiels zu einer sinnvollen Handlung. Alle an der Spielsituation beteiligten Kinder müssen hierfür - wenn auch nicht zwingend bewusst - in der Lage sein, zu verfolgen, dass die jeweils geltenden Spielregeln sowohl den eigenen als auch fremden Handlungen zugrunde liegen.

Rakoczy (2008, S. 1195) wählt im Gegensatz dazu einen anderen Erklärungsansatz. Ihm zufolge liegt dieser kindlichen Spielform die Fähigkeit zugrunde, institutionelle Regeln zu bilden und ihnen entsprechend zu handeln:

In contrast to brute facts "out there," institutional facts hold only by virtue of a social, conventional practice and have the logical form " $\mathrm{X}$ counts as a $\mathrm{Y}$ in a context C." Among the standard examples are money ("This slip of paper counts as money in our currency area") and rule games ("This piece of wood counts as a king in chess" or "Moving the piece of wood in this way counts as attacking in chess"). [...] Institutional facts essentially involve normative, deontic aspects: Given that an $\mathrm{X}$ counts as a $\mathrm{Y}$ in a certain context $\mathrm{C}$, some acts with $\mathrm{X}$ in $\mathrm{C}$ are appropriate and others are inappropriate, mistakes. For example, given that a piece of wood counts as a king in a game of chess, it is appropriate to perform certain moves with it (i.e., move one field in any direction), a mistake to make certain other moves (e.g., move more than one field in any direction), and highly inappropriate to use it as a piece of firewood. Analogously, in the case of joint pretend play, when we pretend that milk was poured from a bottle into a cup, in the context of the pretence the cup now counts as full of milk. It is thus appropriate to pretend to drink from the cup and say "Delicious milk" (or "Disgusting! Milk!"), but it is a mistake to call the cup empty or to say it contains whiskey.

Im Kontext des Fiktionsspiels erkennen Kinder an, dass bestimmte Spielregeln imaginative Inhalte generieren, ein Spielpartner also in seiner Vorstellung z. B. ,mit einem Bären kämpft‘, während er in Wirklichkeit einen Baumstumpf mit einem Ast schlägt. Sie generieren in jeder Spielsituation einen bestimmten Interpretationsrahmen für die eigene Interaktion und Wahrnehmung und für die Interpretation der Handlungen und Wahrnehmungen anderer 
Spielteilnehmer. Mit diesem Entwicklungsschritt ist damit die kognitive Grundlage für weitere Fähigkeiten gelegt, die es verlangen Gegenstände und Wahrnehmung in eine flexible, situationsabhängige Relation zur Realität zu stellen (ebd. S. 1199).

\section{Vorläufiges Fazit: Was ist, ToM‘?}

Der Begriff ,ToM` bezeichnet eine Fähigkeit, die - so ist in den Vergangenen Abschnitten deutlich geworden - eine ganze Reihe von sozialen und kognitiven Leistungen ermöglicht. Die Besonderheit, die diese Fähigkeiten miteinander verbindet, ist, dass sie uns Handlungsund Situationsinterpretationen ermöglichen, die nicht ausschließlich auf ,sichtbare‘ physikalische Gegebenheiten beruhen. Vielmehr verstehen wir unsere Welt und die Handlungen anderer mit Hilfe (angenommener oder bekannter), unsichtbarer' mentaler Haltungen (z. B. Wünsche, Überzeugungen, Wissen und Intentionen) und psychischer Vorgänge (z. B. Imagination und Wahrnehmung). Die Fähigkeit des Menschen zur Attribution von nicht oder nur indirekt sichtbaren inneren Haltungen und Vorgänge optimiert dabei wichtige Prozesse menschlichen Handelns, Lernens und Interagierens.

Die Frage, was die ToM eigentlich ist, ist in den vorangehenden Abschnitten synonym mit der Frage ,Was kann ein gesunder Erwachsener dank seiner ToM leisten?` behandelt worden. Es ist eine allgemein akzeptierte Annahme der kognitiven Literaturwissenschaft, dass Leser ihre ToM-Fähigkeiten in vergleichbarer Form für das Verständnis realer wie fiktiver Kontexte nutzen (Palmer 2004; Zunshine, 2006; Herman, 2013). Lisa Zunshines wichtigstes Argument für die Richtigkeit dieser Annahme lautete, dass Menschen (z. B. Kinder mit einer autistischen Entwicklungsstörung), deren ToM-Fähigkeiten unterentwickelt sind, ähnliche Schwierigkeiten mit dem Verständnis realer wie fiktiver Szenarien haben (Zunshine, 2006, S. 9 f.). Wie bereits zuvor angemerkt, stützt dieses Argument lediglich die Annahme, dass die ToM mit hoher Wahrscheinlichkeit für das Verständnis (fiktiver) Menschen und ihrer Interaktionen eine vergleichbare Funktion wie im Rahmen (realer) sozialer Interaktionen übernimmt. Auf diesem Weg lässt sich nicht im Detail und umfassend klären, welche Funktionen die ToM während der kognitiven Rezeption literarischer Texte übernimmt. Diese Funktionen könnten ggf. über die Zuschreibung mentaler Haltungen auf der Ebene der Fiktionswelt hinausgehen. Um sich der Beantwortung dieser Frage anzunähern, ist es wichtig, eine Vorstellung davon zu gewinnen, welche kognitiven Prozesse und Mechanismen den unterschiedlichen Fähigkeiten, die mit der , ToM` assoziiert werden, zugrunde liegen. Um 
diese Prozesse noch genauer charakterisieren zu können, möchte ich den Fokus daher auf denjenigen Bereich der Kognitionsforschung lenken, der sich mit der kognitiven Funktionsweise der ToM beschäftigt. Die Frage ,Was ist ToM?` wird im Folgenden also zu der Frage ,Wie funktioniert die ToM?` umformuliert. Ein erster Schritt auf diesem Weg ist es, den bereits bestehenden Diskurs über die Funktionsweise der ToM, an dem nicht nur Psychologen, sondern auch Anthropologen und Philosophen teilnehmen, aufzugreifen. Hier wird diskutiert, ob sich an Hand der offensichtlichen Fähigkeiten des Menschen erkennen lässt, ob sich konkrete kognitive Mechanismen identifizieren lassen, die ihr zugrunde liegen könnten.

Es ist dabei nach wie vor offen, ob die mit der ToM assoziierten sozialen Leistungen des Menschen (a) auf ToM-spezifischen Mechanismen beruhen oder ob sie (b) durch eine Reihe allgemeiner kognitiver Ressourcen ermöglicht wird, die nicht ausschließlich der Funktionalität der ToM dienen. Es ist ebenfalls denkbar, dass beide Annahmen zutreffen und es ist interessant, die unterschiedlichen Möglichkeiten dahingehend zu analysieren, ob sich unter der Prämisse, die jeweiligen Annahmen seien wahr, Schlüsse auf die Rolle der entsprechenden kognitiven Mechanismen im Rahmen der literarischen Rezeption ziehen lassen. Zugunsten der Übersichtlichkeit möchte ich damit beginnen, die unterschiedlichen Ansätze zur Erklärung der Funktionsweise der ToM kurz vorzustellen, bevor ihre mögliche Rolle in der literarischen Rezeption in den Fokus rückt.

\section{Wie funktioniert die ToM? - Grundlegende kognitive Mechanismen}

\subsection{Die kognitive Repräsentation mentaler Haltungen}

\section{ToM als kognitive ,naive' Theorie}

Premack und Woodruff umschrieben die ToM im Rahmen ihrer ursprünglichen und forschungsbegründenden Definition aus dem Jahr 1978 als ,ein System von Schlussfolgerungen` über eigene und fremde mentale Haltungen und Vorgänge. Sie schreiben außerdem: „A system of inferences of this kind is properly viewed as a theory, first, because such states are not directly observable, and second, because the system can be used to make predictions, specifically about the behavior of other organisms“ (Premack \& Woodruff, 1978, S. 515). Diese Annahme spiegelt sich in der ursprünglichen Wahl der Terminologie (,Theory ‘ of 
Mind) wider. Premack und Woodruff gehen davon aus, dass die ToM auf einer kognitiven Leistung basiert, die mit der Bildung einer ,naiven“ Theorie zu vergleichen ist. Ihr Grundgedanke lautet, dass Menschen über eine Reihe von Informationen über die Beschaffenheit und Funktionalität des menschlichen Geistes verfügen, also z. B. abstrakte Konzepte über die Funktion und Eigenschaften verschiedener mentaler Haltungen besitzen. Mit Hilfe dieser Konzepte, den Informationen über eine bestimmte gegebene Situation oder Handlung und einer Reihe abstrahierender Schlussfolgerungen können sie ein Bild - eine Theorie - über die eigene mentale Welt oder diejenige eines Gegenübers erstellen. Die über Schlussfolgerungen gewonnenen Erkenntnisse können wir wiederum dazu nutzen, zukünftige Handlungen und innere Vorgänge vorherzusagen und Schlüsse über vergangene und gegenwärtige mentale Haltungen zu ziehen (Carruthers, 1996, S. 22). Laut den Verfechtern des TheorieAnsatzes funktioniert die ToM des Menschen also mittels einer kognitiven Systematik. Diese Systematik synthetisiert das Wissen über die funktionalen Eigenschaften von unterschiedlichen mentalen Kategorien (z. B. ,Überzeugung`vs. ,Wissen` vs. ,Wunsch`) von beobachteten Personen mit dem konzeptuellen Wissen über bestimmte Regeln und Gesetze zum allgemeinen menschlichen Verhalten im Rahmen eines kognitiven Auswertungsverfahren. Auf dieser Basis lassen sich verschiedene Schlussfolgerungen, z. B. die Vorhersage eines bestimmten Verhaltens generieren. Wellman (2001, S. 95) schreibt:

[...] the states being inferred - hopes, intentions, wishes, fantasies - must operate together in several ways. We assume the connectedness among such mental terms and descriptions. In the extreme, therefore, mental states [...] seem to be theoretical constructs whose meanings partake of a variety of relationships within a web of constructs. Attribution of a target mental state depends at least as much on the target's presumed coherence with other states as it does on an anchoring in observation or introspection.

Ein Beobachter, der davon ausgeht, dass Wünsche sich in zielgerichteten Handlungen niederschlagen, die der Erfüllung dieser Wünsche dienen und durch Überzeugungen über die Beschaffenheit der Welt gelenkt werden, kann also 1. basierend auf den ihm bekannten mentalen Haltungen eines anderen Handlungen antizipieren oder 2. basierend auf der Beobachtung einer konkreten Handlung Rückschlüsse über die mentalen Haltungen der beobachteten Person ziehen (Gergely \& Csibra, 2003). Wenn eine Person A weiß, dass eine Person B gern Eiscreme essen möchte, und auch weiß, dass B der Überzeugung ist, dass es an einem bestimmten Ort einen Eisstand gibt, ist A in der Lage anzunehmen, dass sich B mit hoher Wahrscheinlichkeit in Kürze zu diesem bestimmten Ort begeben wird. Wenn A weiß, dass es an einem bestimmten Ort einen Eisstand gibt, und B dabei auf dem Weg zu diesem Ort 
beobachtet, kann er annehmen, dass B mit einiger Wahrscheinlichkeit Eiscreme kaufen möchte.

Die Annahme, die ToM basiere auf einer Reihe abstrakter Konzepte, die ein Mensch ab einem bestimmten Zeitpunkt seiner Entwicklung , besitzt' und die es ihm erlauben eine ,naive“ Theorie über die mentale Welt eines Gegenübers zu erstellen, ist nicht unproblematisch. Würde die ToM tatsächlich so funktionieren, dann müssten Kinder etwa zum gleichen Zeitpunkt ihrer Entwicklung in der Lage sein, eigene und fremde mentale Haltungen und Vorgänge zu verstehen, denn die Konzepte, die sie von nun an besitzen, können gleichermaßen für das Verständnis eigener und fremder mentaler Haltungen genutzt werden. Dabei stellt sich die Frage, ob und in welchem Ausmaß der Besitz solcher abstrakten Konzepte es Kindern nicht eigentlich erlauben müsste, die Eigenschaften und Funktionen von mentalen Haltungen bewusst zu reflektieren. Es ist außerdem offen, wie Kinder die notwendigen abstrakten Konzepte erwerben. Die Frage, die im Kontext der Theorie-Theorie schwierig zu beantworten ist, lautet genauer gesagt: Wie können Kinder im Verlauf ihrer Entwicklung kognitive Konzepte über - an sich unsichtbare - mentale Haltungen gewinnen, wenn diese nicht direkt zugänglich und damit für das kindliche Lernverhalten verschlossen sind? Eine Hypothese lautet, dass es ein besonderer ,ToM-Mechanismus ' gibt, der in einem gewissen Alter reift. Ein solcher Mechanismus könnte die Grundlage für alle mit der ToM assoziierten Fähigkeiten bilden.

\section{Der ,Theory-of-Mind-Mechanismus ${ }^{6}$}

Gestützt wird die Annahme eines solchen ToM-Mechanismus durch Erkenntnisse aus der ASD-Forschung. Leslie (2000, S. 1236) schreibt:

For the young brain to move attention from behavior to the mental state from which the behavior issues, appropriate processing mechanisms must deploy a system of representation capable of representing mental states. I call this system of representation the M-representation, and the associated processing mechanisms the theory of mind mechanisms (ToMM). [...] The ToMM is a mechanism of selective attention, it operates postperceptually, it operates spontaneously whenever an agent's behavior is attended [...].

Der wichtige Befund der ASD-Forschung lautet, dass Kinder, die an einer der verschiedenen Ausprägungsstufen der Autismus-Spektrum-Störung leiden, ToM-spezifische Defizite zeigen. Trotzdem viele kognitive Ressourcen der allgemeinen Intelligenz unbeeinträchtigt sind, 
scheinen sie Schwierigkeiten zu haben, die mentalen Haltungen und inneren Vorgänge anderer zu interpretieren,. Sie zeigen diese Defizite z. B. auch dann, wenn die Leistungen der allgemeinen Intelligenz, gemessen an ihrem Intelligenzquotienten normal entwickelt sind (Baron-Cohen, Leslie \& Frith, 1985). Die Beobachtung einer scheinbar so selektiven Störung, so die Argumentation Leslies (Leslie, 1987, S. 417), lässt auf die Existenz eines besonderen ,ToM-Moduls‘ schließen, das im Fall einer autistischen Entwicklungsstörung unterentwickelt bleibt. Diese Unterentwicklung ist wiederum die Ursache für die Einschränkungen der betroffenen Kinder in der sozialen Interaktion mit anderen (Leslie, 1992, S. 21).

Gesunde Kinder beginnen mit etwa 18 Monaten die für das Fiktionsspiel typischen Verhaltensweisen zu zeigen. Leslie sieht darin einen Hinweis für die Reifung des ToM-Mechanismus, da die Kinder von nun an offenkundig zwischen nicht ausschließlich realitätsbezogenen eigenen und fremden Handlungen und ausschließlich realitätsbezogenen Handlungen unterscheiden können. Diese Fähigkeit lässt sich Leslie zufolge über einen Mechanismus erklären, den er in einer frühen Arbeit ,Entkopplungsmechanismus“ (decoupling mechanism) nennt (Leslie, 1987). Wenn Kinder während einer Episode des Fiktionsspiels bestimmte Requisiten spielerisch zweckentfremden, so die Überlegung, dann müssen sie über einen kognitiven Mechanismus verfügen, der die Trennung (,Entkopplung') der tatsächlichen Identität eines Gegenstandes von seiner fiktiven Identität ermöglicht (ebd., S. 416 f.). Dabei entstehen primäre und sekundäre kognitive Repräsentationen des Gegenstandes. Primäre Repräsentationen sind solche, die reale Objekte und Personen unmittelbar repräsentieren und damit einen direkten Bezug zur realen Welt besitzen. Die Repräsentation eines spielerisch zweckentfremdeten Gegenstandes im Kontext des Fiktionsspiels muss sich von der primären Repräsentation desselben Gegenstandes qualitativ unterscheiden oder mit einem mentalen ,Index ‘ versehen sein, da ansonsten die Gefahr bestünde, dass das Wissen über die Realität und damit das Lernverhalten des Kindes gestört würde. Leslie nennt diese kognitiven Repräsentationen ,sekundäre' Repräsentationen, da sie sich zwar z. T. auf Objekte der realen Welt beziehen, diese aber mit alternativen (imaginativen) Eigenschaften ausgestattet werden müssen. Die Fähigkeit, das spielerische Verhalten anderer zu erkennen, lässt darauf schließen, dass im Zuge des Fiktionsspiels darüber hinaus nicht ausschließlich Repräsentationen von Gegenständen, sondern außerdem die Funktion und Bedeutung einer bestimmten mentalen Haltung oder Einstellung gegenüber dem Spielpartner und den genutzten Requisiten prozessiert wird. Um an einer Episode des FS teilnehmen zu können müssen Kinder also nicht nur verstehen, dass ein bestimmter Gegenstand in dieser Situation bestimmte fiktive Eigenschaften besitzt, sondern ebenfalls verarbeiten, dass der Spielkamerad ein Objekt in 
einer Form repräsentiert, die nicht dessen primärer Repräsentation entspricht. Sie müssen verstehen - oder zumindest kognitiv registrieren, dass die auf dieses Objekt bezogenen Handlungen im Kontext der Fiktionsspiel-Situation, und nicht in einem realitätsbezogenen Kontext $\mathrm{zu}$ verstehen sind. Leslies Schlussfolgerung hinsichtlich des kindlichen Fiktionsspiels lautet also: „Pretend representations [...] are in effect not representations of the world but representations of representations. For this reason I shall call them second order or [...] metarepresentations. “13 Leslie unterstellt hier, dass Kinder, die im Alter von anderthalb Jahren am FS teilnehmen, Repräsentationen von Repräsentationen - also ,Meta'Repräsentationen erstellen können. Genau wie auch im Fall des Theorie-Ansatzes stellt sich hier allerdings die Frage, ob und in welchem Ausmaß es uns dank solcher Metarepräsentationen möglich ist, die Eigenschaften mentaler Haltungen bewusst zu reflektieren. Vor allem mit Blick auf das junge Alter von Kindern, die beobachtete Handlungen bereits auf innere Vorgänge zurückführen können, ist es allerdings schwierig zu unterstellen, sie besäßen bereits so etwas, wie ein explizites, Wissen“ über die Natur und Funktion mentaler Haltungen. Kritiker dieser Annahme (z. B. die Verfechter des alternativen Simulations-Ansatzes) stellen sich die Frage, ob es notwendig und sinnvoll ist, jungen Kindern solch komplexe kognitive Leistungen zu unterstellen, um die mit der sich entwickelnden ToM zusammenhängenden psychologischen Kompetenzen zu erklären. ${ }^{14}$ Es sei gar nicht notwendig, von einem solchen Mechanismus und dem Vorhandensein abstrakter mentaler Konzepte auszugehen um z. B. die entsprechenden Verhaltensweisen im Rahmen des Fiktionsspiels zu erklären, heißt es z. B. bei Harris. ${ }^{15}$ Es sei ebenso möglich, dass Kinder die Handlungen anderer im Kontext des Fiktionsspiels lediglich als eine bestimmte Verhaltensweise anerkennen und die eigenen Handlungen dementsprechend anpassen. ${ }^{16}$

Ein weiteres Argument gegen Leslies Argumentation für den Reifungsprozess eines speziellen ToM-Mechanismus im Alter von anderthalb Jahren liefern die Ergebnisse jüngerer Habituationsstudien der Entwicklungspsychologie. Sie zeigen, dass schon sehr viel jüngere Kinder (8-10 Monate) menschliche Handlungen interpretieren, indem sie ihnen handlungsmotivierende mentale Haltungen (Handlungsziele und Intentionen) unterstellen (Király,

\footnotetext{
${ }^{13}$ Hervorhebung durch den Autor.

${ }^{14}$ Eine systematische und ausführliche Gegenüberstellung des Theorie-Ansatzes und weiterer Schulen steht mit dem ersten Kapitel in Alvin Goldmans Arbeit Simulating minds (2006a, S. 10 f.) zur Verfügung. Ich stelle den Simulations-Ansatz etwas ausführlicher im Abschnitt 4.4 ToM als kognitive Simulation dieses Kapitels ausführlicher vor.

${ }^{15}$ Für eine ausführliche Diskussion des Problems vgl. Harris (1992).

${ }^{16}$ Eine solche behavioristische Beschreibung des kindlichen Spielverhaltens ist bei Nichols und Stich (2004; 2003) impliziert.
} 
Jovanovic, Prinz, Aschersleben \& Gergely, 2003). Das könnte bedeuten, dass der von Leslie angenommene ToM-Mechanismus, der es gestattet sowohl primäre als auch sekundäre Repräsentationen zu bilden, eventuell zu einem sehr viel früheren Entwicklungszeitpunkt zu reifen beginnt und sich über einen längeren Zeitraum hinweg weiterentwickelt. Es ist aber ebenso denkbar, dass die ToM nicht dank eines solchen exklusiven ToM-Mechanismus, sondern durch das Zusammenwirken allgemeiner kognitiver Kompetenzen ermöglicht wird. In diesem Fall wären Entwicklungsfortschritte in verschiedenen Bereichen der Kognition dafür verantwortlich, dass Kinder mit steigendem Alter zunehmend komplexe soziale Aufgaben meistern können. Im Hinblick auf diese Überlegung stellt sich die Frage, welche Bereiche der allgemeinen menschlichen Kognition beim Lösen der unterschiedlichen ToM-Aufgaben involviert sind. Um diese Frage zu beantworten, ist ein Blick auf die grundlegenden Prinzipien der menschlichen Kognition hilfreich. Es gilt zu klären, was eigentlich damit gemeint ist, wenn von einer ,kognitiven Repräsentation` die Rede ist.

\section{Situationsrepräsentation mittels ,mentaler Modelle`?}

In den Kognitionswissenschaften werden kognitive Prozesse traditionell als algorithmische Berechnungen eines ,Codes` beschrieben, der die Informationen aus der Umwelt des Menschen symbolisch ,repräsentiert ' (Shapiro, 2011, S. 2). Die menschliche Kognition ist - dieser Ansicht entsprechend - mit einem Computer- oder Rechenprogramm vergleichbar. Dabei werden zum einen im Zuge von Wahrnehmungsvorgängen Reize aus der Umwelt aufgenommen (Input) und in solcher Form verarbeitet und modifiziert, dass bestimmte (körperliche, geistige oder emotionale) Reaktionen (Output) ausgelöst werden. Zum anderen wird angenommen, dass perzeptive Prozesse zur Bildung von kognitiven Repräsentationen führen. Mit Hilfe solcher Repräsentationen ist der Mensch in der Lage, kognitive Inferenzen über seine Umwelt zu bilden und sich in Situationen, in denen Informationen hergeleitet werden müssen, zurechtzufinden. Der Mensch kann die aufgenommenen Informationen dabei laut Johnson-Laird nicht nur in einer, sondern in verschiedenen Formen repräsentieren (Johnson-Laird, 1983, S. 165). Diese Formen sind: ,[...] propositional representations which are strings of symbols that correspond to natural language, mental models which are structural analogues of the world, and images which are the perceptual correlates of models from a particular point of view“ (ebd.). Alle diese Repräsentationsformen basieren letztlich auf einer kognitiven Codierung von Informationen aus der Umwelt. Die Differenz besteht jedoch in den unterschiedlichen Modi der Inferenzenbildung, die mit Hilfe der jeweiligen Repräsentationsform möglich sind. Propositionale Repräsentationen gestatten regelbasierte 
kognitive Inferenzen (rule-based cognitive reasoning) über syntaktische Konnektive (,if ${ }^{*}$, ,or ${ }^{6}$, , $a n^{6}$ und ,not ${ }^{\star}$ ) (Johnson-Laird, Byrne \& Schaeken, 1992, S. 418). Abstrakte, regelbasierte Schlüsse sind jedoch nicht in allen denkbaren sozialen Szenarien, in denen Inferenzen gebildet werden müssen, um eine Situation adäquat interpretieren zu können, möglich oder hilfreich (ebd., S. 419). Daher schlagen Johnson-Laird et al. vor, dass Menschen (im Besonderen in komplexen sozialen Interaktionssituationen) kognitive mentale Modelle ihrer Umwelt bilden, um mit ihrer Hilfe Schlussfolgerungen über die jeweilige Situation ziehen zu können. Solche kognitiv gebildeten Modelle repräsentieren die Objekte und Ereignisse der Welt mit den jeweils zugeordneten Eigenschaften und Relationen untereinander und basieren auf perzeptiv gewonnenen Informationen, vorhandenem Wissen und bereits geleisteten Inferenzen:

Semantic procedures construct models of the premises, formulate parsimonious conclusions from them, and test their validity by ensuring that no alternative models of the premises refute them. [...] Our view is that models have a structure that corresponds directly to the structure of situations. Each individual in a situation is represented by a corresponding mental token, and the properties of individuals and the relations among them are likewise modeled in an isomorphic way [...]. (Ebd.)

Mentale Modelle sind dynamisch und können - wenn dazu Anlass oder die Notwendigkeit besteht - an die sich verändernden Umweltparameter angepasst werden (Kintsch, 1998, S. 14).

Josef Perner argumentiert, dass die kognitiven Prozesse, die den verschiedenen Fähigkeiten der ToM zugrunde liegen, mit Hilfe dieser Theorie der mentalen Modelle zu erklären sind. Kinder durchlaufen im Zuge ihrer allgemeinen kognitiven Entwicklung seiner Ansicht nach verschiedene Entwicklungsstufen. Dabei ist es ihnen zunächst lediglich möglich, primäre Repräsentationen bzw. einfache Modelle von Objekten und Personen in der Welt zu erstellen. Perner schreibt: „At this stage of development the infant's understanding of the mind is restricted to selective attention to behavior (as an expression of mentality) and perhaps to detecting behavioral regularities reflecting the common mental root of different behaviors“ (Perner, 1991, S. 284). Sekundäre Repräsentationen, die mit fortgeschrittenen kognitiven Kompetenzen möglich sind, bestehen laut Perner aus multiplen Modellen, die miteinander abgeglichen werden können. Diese Modelle können nun nicht mehr nur die unmittelbare gegenwärtige Situation, sondern ebenfalls vergangene, zukünftige oder hypothetische Situationen repräsentieren. Kinder, die am Fiktionsspiel teilnehmen, können auf diese Weise 
Realität und Fiktion trennen, indem sie verschiedene Repräsentationen der Welt bilden. Die kognitiven Modelle von Situationen, die im Zuge einer Episode des Fiktionsspiels entstehen, sind laut Perner also nicht solche, die die Welt wie sie tatsächlich ist repräsentieren, sondern Modelle der Welt, wie sie hypothetisch sein könnte (ebd., S. 59). Perner schreibt: „Not until this point can the child start to understand people as mentally aiming at something nonexistant" (ebd., S. 284). Neben dem kindlichen Fiktionsspiel lässt sich auf diesem Weg auch die Fähigkeit, die gängigen false-belief-Aufgaben zu lösen, einsichtlich begründen. Kinder müssen im Rahmen der standardisierten ,Maxi-Aufgabe‘ die Frage beantworten, an welcher Stelle ein fiktiver Charakter (Maxi) auf Grund seiner eigenen (falschen) Überzeugung nach einer Tafel Schokolade suchen wird, die in seiner Abwesenheit zuvor verlegt worden war. Um diese Frage richtig zu beantworten, ist es notwendig, das eigene Wissen über den Verbleib der Schokolade mit der fremden Perspektive von Maxi und seinem individuellen $\mathrm{Zu}-$ gang zu Informationen und Wissen abzugleichen. Ein Kind, das sowohl die Realität, die es selbst wahrnimmt, als auch Maxis subjektive (fehlerhafte) Repräsentation der Welt kognitiv modellieren kann, ist in der Lage, Maxis erfolglose Suche am falschen Ort vorherzusagen (Carruthers \& Smith, 1996, S. 2).

Diese Beschreibung der Funktionsweise der ToM wirft eine Frage auf: Wenn die menschliche Kognition mit der algorithmischen Berechnung eines Codes zu vergleichen ist, wobei an Hand von isomorphen Modellen Inferenzen über die Umwelt, generiert' und darauf aufbauend adäquate Reaktionen berechnet werden können - was unterscheidet den Menschen dann von einer (zugegebenermaßen kunstfertigen) Maschine? Gesunde Erwachsene besitzen dank ihrer ToM u. a. die Fähigkeit zur bewussten Wahrnehmung bzw. Reflektion eigener und fremder mentaler Haltungen und innerer Vorgänge. Das bedeutet, sie verfügen über ein ,Selbst'-Bewusstsein im eigentlichen Wortsinn. Im Kontext der Modell-Theorie lässt sich dieses Phänomen durch die Eigenschaft der Rekursivität mentaler Modelle erklären. Mit Rekursivität ist gemeint, dass nicht nur Modelle von der (fremden) Umwelt, sondern auch Modelle des eigenen Selbst und der eigenen Bewusstseinsvorgänge erstellt werden können. Perner integriert diesen Gedanken in seine Vorstellung der Entwicklung der ToM. Ihm zufolge erlangen Menschen im weiteren Verlauf der Entwicklung zusätzlich die Fähigkeit, Repräsentationen von Modellen - also Metarepräsentationen im eigentlichen Sinne - zu bilden. Menschen können daher nicht nur innere Vorgänge und Handlungen in Relation zueinander setzen, sondern besitzen von nun an die notwendigen kognitiven Fähigkeiten, um die menschliche Kognition und die mentale Weil eines anderen als solche und auch die eigene Wahrnehmung dieser mentalen Welt zu reflektieren. Demnach ist im Zuge der kognitiven 
Entwicklung ein qualitativer Entwicklungssprung in der menschlichen ToM zu verzeichnen, die sich bei jungen Kindern als ,Theorie des menschlichen Verhaltens ${ }^{\star}$ und bei gesunden Erwachsenen als ,Theorie des menschlichen Geistes' umschreiben lässt (Perner, 1991, S. 124). Allerdings lässt sich auch im Zuge dieser theoretischen Konzeption der ToM schwer dagegen argumentieren, dass für die Repräsentation von unterschiedlichen mentalen Haltungen zusammen mit dem Wissen über ihre Bedeutung und Funktion - wie von den TheorieTheoretikern angenommen - eine bestimmte Menge propositionalen Wissens über einzelne Kategorien mentaler Haltungen notwendig ist:

Understanding representation initiates a restructuring of mental concepts such as ,knowledge' within a representational theory of mind. [...] However, the representational view does not replace the original situation theory (mental states as propositional attitudes) but only extends it. Even our adult commonsense psychology is not cognitive at heart, but is capable of taking a cognitive, representational stance when needed. (Perner, 1991, S. 284)

Perners zufolge entspricht die Entwicklung der ToM einer schrittweisen qualitativen ,Metamorphose' der Fähigkeiten zur kognitiven Repräsentation von Gegenständen, Situationen und Personen mit Hilfe von mentalen Modellen. Eine genaue Beschreibung der kognitiven Mechanismen, mit Hilfe derer die entsprechenden Inferenzen an Hand der Modelle geleistet werden, lassen sich mit diesem Ansatz jedoch nicht identifizieren.

Im Rahmen einer Metastudie konnte Wellman feststellen, dass allgemeine, nicht ToM-spezifische kognitive Ressourcen für die Funktionalität der ToM eine Rolle spielen. Hier zeigte sich, dass unterschiedliche false-belief-Testdesigns, die unterschiedliche Prozessierungsanforderungen an die Testteilnehmer stellten, den gemessenen Alterseffekt für das Lösen der entsprechenden Aufgabe beeinflussen können. Aufgaben mit geringeren Anforderungen an die allgemeine kognitive Informationsverarbeitung (z. B. an das Arbeitsgedächtnis oder sprachliche Fähigkeiten) gestatten es Kindern bereits zu einem früheren Zeitpunkt in ihrer Entwicklung die jeweiligen Aufgaben korrekt zu lösen (Wellman, Cross \& Watson, 2001, S. 674). Im Folgenden Abschnitt soll daher an Hand von ausgewählten entwicklungspsychologischen Studien hinsichtlich zweier verschiedener kognitiver Bereiche - den sogenannten ,Exekutiven Funktionen“ und der Sprachentwicklung - die Bedeutung allgemeiner Informationsverarbeitung für die ToM des Menschen genauer dargestellt werden. 


\subsection{Die Rolle der kognitiven ,Exekutiven Funktionen' für die ToM}

In die Kategorie kognitiver Ressourcen, die als ,Exekutive Funktionen“ (nachfolgend EF) bezeichnet werden, fallen eine Reihe einzelner Mechanismen zur Handlungsplanung, Verhaltensregulierung, kognitiven Flexibilität, Fehlerdetektion und -korrektur, Inhibitionskontrolle und nicht zuletzt auch das Arbeitsgedächtnis. Einige dieser Mechanismen stehen in einem engen korrelativen Zusammenhang mit den Aufgaben der ToM-Skala, wie im Rahmen zahlreicher Studien nachgewiesen werden konnte. Außerdem teilt sich diese Kategorie kognitiver Fähigkeiten einen gemeinsamen Entwicklungszeitraum mit der Ausbildung sprachlicher Fähigkeiten und der Entwicklung kognitiver Konzepte verschiedener mentaler Haltungen. Darüber hinaus sind auch in diesem Fall gemeinsame Defizite in pathologischen Fällen wie in (unterschiedlich ausgeprägten) Formen der Autismus-Spektrum-Störung oder bspw. Patienten mit Frontotemporaler Demenz (FTD) zu verzeichnen (Lough, Kipps, Treise, Watson, Blair \& Hodges, 2006). Zusammen mit dem Befund, dass die Manipulation des Schwierigkeitsgrades z. B. von false-belief-Aufgabenstellungen die Leistungsfähigkeit jüngerer Kinder beim Lösen typischer ToM-Aufgaben steigert (Wellman et al., 2001) sprechen diese Indizien dafür, dass den kognitiven Ressourcen der EF auch im Rahmen der ToM eine zentrale Funktion zufällt. Mit Hilfe bildgebender Verfahren und anderer Versuchsanordnungen ließ sich ein signifikanter Zusammenhang zwischen den EF und der ToM vielfach nachweisen (Saxe, Schulz \& Jiang, 2006). Die gemessenen, überwiegend sehr robusten Korrelationen erklären jedoch nicht zufriedenstellend, was den Zusammenhang zwischen diesen beiden Fähigkeitskomplexen bedingt. Es ist möglich, dass mangelnde Fähigkeiten in bestimmten Bereichen der EF dazu führen, dass bereits vorhandene Kompetenzen zum Verständnis mentaler Vorgänge innerhalb der gängigen Versuchsdesigns schlicht verdeckt werden. Denkbar wäre beispielsweise, dass fiktive Szenarien, wie z. B. die Maxi-Geschichte, eine zu hohe Anforderung an das Arbeitsgedächtnis stellen und die Kinder mit der Aufrechterhaltung aller relevanter Informationen überfordert sind. Kinder, deren Arbeitsgedächtnis noch nicht weit genug entwickelt ist, können in diesem Fall möglicherweise vorhandene ToM-Kompetenzen nicht zum Ausdruck bringen, weil es ihnen an anderen Kompetenzen (wie z. B. dem Arbeitsgedächtnis) mangelt.

Alle Aufgaben zur falschen Überzeugung und zum vorsätzlichen Täuschen beinhalten zusätzlich zu den Anforderungen an das Arbeitsgedächtnis einen hohen Anspruch an die ,Inhibitorische Kontrolle' (nachfolgend IK). Die IK ist dafür verantwortlich, einen spontanen Handlungsimpuls, der aufgrund eines vordergründigen oder dominanten Stimulus ausgelöst 
wird, zu unterdrücken. Wenn eine false-belief-Aufgabe gelöst werden soll, muss die sichtund spürbare reale Situation zu Gunsten einer nicht unmittelbar einsichtigen und daher weniger salienten fehlerhaften kognitiven Repräsentation der Welt - so wie sie der Betroffene wahrnimmt - unterdrückt werden. Ein einfaches Beispiel, nämlich die Leistungen von Kindern im ,Smartiestest ‘ unterstreicht die Zentralität der IK im Rahmen der standardisierten false-belief-Aufgaben. Hier dürfen Kinder den Inhalt einer Smartiesschachtel untersuchen und finden dabei heraus, dass es die Schachtel nicht mit Smarties, sondern mit anderen Gegenständen (z. B. Stiften) gefüllt ist. Sie müssen anschließend die Frage beantworten, zu welcher Einschätzung ein Spielkamerad, der während der ersten Phase des Experiments keinen Zugang zur Schachtel hatte, gelangen würde. Kinder unter dreieinhalb Jahren beantworten diese Frage in der Regel kongruent zu ihrem eigenen Wissensstand, während Vierjährige und ältere Kinder in der Lage sind, ihr eigenes und damit salientes Wissen um den tatsächlichen Inhalt zu Gunsten des fremden Wissensstandes zu unterdrücken und die Frage korrekt zu beantworten. ${ }^{17}$ Es ist durchaus denkbar, dass sogar die Kinder in der jüngeren Versuchsgruppe in der Lage wären, den fremden Wissensstand korrekt zu repräsentieren, dann aber an der Ausführung der Aufgabe scheitern, weil sich der Stimulus des tatsächlich in der Schachtel befindlichen Gegenstandes für die noch nicht ausgereifte IK als zu dominant darstellt.

Um ein detaillierteres Bild des Zusammenhangs zwischen EF und ToM-Bildung zeichnen zu können und damit Einblicke in die für die mangelhafte Leistung jüngerer Kinder ausschlaggebenden Mechanismen zu erlangen, wurden mit Bezug auf die oben dargestellte Vermutung verschiedene Aufgaben zur IK getestet. Diese lassen sich grob in zwei Kategorien - die der ,delay tasks ‘ und die der , conflict tasks ${ }^{6}$ - einteilen. Letztere erzielten über viele verschiedene Tests hinweg stärkere Effekte und Korrelationen mit den Aufgaben zur falschen Überzeugung und sind damit ein Indiz für den Mechanismus, auf den es im Hinblick auf die ToM-Bildung besonders anzukommen scheint. Ein typisches und in vielen Studien verwendetes Beispiel für eine Aufgabe aus der Reihe der delay-tasks ist die ,Flüsteraufgabe“ (Kochanska, Murray, Jacques, Koenig \& Vandegeest, 1996). Kinder müssen hier - im Flüsterton - verschiedene bekannte und unbekannte Cartoon-Charaktere benennen. Eher unbekannte Charaktere (Huckle, Petunia, Elmer Fudd) werden deshalb einbezogen, um die Versuchung der Kinder, die Stimme bei bekannten Gesichtern (Donald Duck, Schneewittchen,

\footnotetext{
${ }^{17}$ Eine ausführliche Beschreibung dieses Testdesigns und der entsprechenden Ergebnisse findet sich in Perner, Frith, Leslie \& Leekam (1989).
} 
Mickey Mouse) zu erheben, deutlich zu steigern. Die Anforderungen an die IK ist im Rahmen dieser Aufgabe besonders hoch, während keine weiteren Teilaspekte der Exekutiven Funktionen involviert sind. Anders verhält es sich mit den Konfliktaufgaben (conflict tasks). Hier müssen Kinder die während der spezifischen Versuchssituation jeweils gültigen Spielregeln (die sich im Verlauf der Tests durchaus ändern können) kontinuierlich im Arbeitsgedächtnis behalten. Bei der ,Bär/Drachen`-Aufgabe sind die jungen Testteilnehmer bspw. angewiesen, den Aufforderungen des ,guten` Bären Folge zu leisten, nicht jedoch denjenigen des ,bösen“ Drachen. Die Herausforderung besteht in diesem Fall darin, einer klaren Aufforderung (durch den negativ konnotierten Drachen) zu widerstehen, wobei sie im Arbeitsgedächtnis behalten müssen, welchen Aufforderungen (des Bären / nicht des Drachen) Folge $\mathrm{zu}$ leisten ist und welcher nicht. Konfliktaufgaben, die gleichzeitig Ansprüche an das Arbeitsgedächtnis und die Inhibitionskontrolle stellen, erzielen durchweg höhere positive Korrelationswerte mit den Aufgaben des ToM-Komplexes als delay-Aufgaben, die entweder allein das Arbeitsgedächtnis oder die Inhibitionskontrolle testen (ebd., S. 498 f.).

Der Konflikt zweier unterschiedlich salienter Stimuli, der die Kapazitäten des Arbeitsgedächtnisses fordert, scheint also die Kompetenz der Kinder beim Lösen der Aufgabenstellungen zur Dekodierung falscher Überzeugung besonders stark zu beeinflussen. Die Schlussfolgerung von Rebecca Saxe und ihrem Team lautet daher: „Belief attribution recruits both brain regions associated with domain-general attention, response selection and inhibitory control, and in addition at least one brain region associated with domain-specific representations of the contents of others' thoughts“" (Saxe et al., 2006, S. 284). Es ist anzunehmen, dass eine funktionierende ToM unterschiedliche kognitive Ressourcen, darunter das Arbeitsgedächtnis, die inhibitorische Kontrolle und Mechanismen zur Repräsentation von Situationen sowie propositionales Wissen über die Funktion und Bedeutung von verschiedenen mentalen Haltungen voraussetzt.

\subsection{ToM und Sprache}

Neben den Korrelationen der bereits zuvor angesprochenen Teilbereiche der EF mit den Kompetenzen beim Lösen von false-belief-Aufgaben fallen in vielen Studien auch Korrelationen mit sprachlichen Fähigkeiten auf. Die Ausbildung von linguistischen Grundfertigkeiten und die Entwicklung der ToM erstrecken sich über den Entwicklungszeitraum der ersten 
fünf Lebensjahre. Die Ergebnisse zahlreicher Studien, die sich der Frage nach einer möglichen Relation zwischen Sprache und ToM gewidmet haben, bestätigen die Korrelation zwischen sprachlichen Fähigkeiten und den Kompetenzen im Hinblick auf die Lösung von kanonischen ToM-Aufgaben. Diese Ergebnisse werden von Studien mit normal entwickelten Kindern als auch von solchen mit klinisch auffälligen Kindern (bspw. Kindern mit einer Entwicklungsstörung des Autismus-Spektrums) dokumentiert (Astington \& Jenkins, 1999; Jenkins \& Astington, 1996; Happé, 1995). Ein genauerer Blick auf die Ergebnisse dieser Studien hilft, den Grund für die gemessenen Korrelationen näher einzugrenzen.

Eine der wichtigsten Fragestellungen, die in den hier relevanten Studien immer wieder gestellt wird, betrifft die Richtung der Beeinflussung zwischen den beiden unterschiedlichen Fähigkeiten, also der sprachlichen Entwicklung und derjenigen der ToM. So ist es denkbar, dass sprachliche Kompetenzen die ToM unterstützen, indem sie notwendige kognitive Strukturen bereitstellen oder vice versa. Außerdem stellt sich die Frage, ob und wenn ja, welche einzelnen Teilbereiche zweier komplexer Phänomene wie der Fähigkeit zur ToM und der menschlichen Sprache in Beziehung zueinander stehen. Um diese Frage zu beantworten, ist es notwendig sprachliche Fähigkeiten und die Fähigkeit der ToM einander nicht nur als ganzheitliche Phänomene gegenüberzustellen, sondern die einzelnen Dimensionen des Eigenschaftspektrums beider Fähigkeiten aufzuschlüsseln.

Die Studien, die dies näher untersuchen, verfolgen teilweise unterschiedliche Erkenntnisziele. Auf der einen Seite wird die Wechselwirkung zwischen den Leistungen beim Lösen der ToM-assoziierten Aufgabenstellungen und Sprache als soziales Medium der Kommunikation genauer untersucht. Hier zeigen die Ergebnisse, dass Kinder, die in größeren Familien, also in einem komplexeren Umfeld sozialer Interaktion aufwachsen, früher bessere Ergebnisse hinsichtlich standardisierter ToM-Aufgaben erzielen (Cutting \& Dunn, 1999). Andere Untersuchungen verfolgen das Ziel, einen generellen Effekt von Sprache als Einflussfaktor auf die kognitiven Prozesse der ToM zu identifizieren. Solche Versuchsdesigns erlauben in der Regel ausschließlich die Erhebung von Daten zum allgemeinen sprachlichen Entwicklungsstand mit einem Schwerpunkt auf dem rezeptiven Sprachverstehen der Kinder. Sie differenzieren nicht zwischen einzelnen Dimensionen (zum Beispiel Semantik und Syntax), die der menschlichen Sprache zu eigen sind. Es liegt allerdings ebenfalls eine Reihe von Studien vor, die dem Ziel dienen, detailliertere Erkenntnisse über den Zusammenhang zwischen ToM und Sprachfähigkeit zu gewinnen. Die folgenden Erläuterungen beschränken sich auf repräsentative Projekte aus diesem Bereich. 
Verschieden Langzeitstudien (z. B. Astington \& Jenkins, 1999) kamen bezüglich der Effektrichtung innerhalb der Korrelation zwischen der allgemeinen Sprachentwicklung und der Fähigkeit zur ToM-Bildung jeweils zu den gleichen Ergebnissen. Die dort gesammelten Daten sprechen für eine Förderung der ToM-Kompetenzen durch vorausgehende sprachliche Fähigkeiten (Lockl, Schwarz \& Schneider, 2004). Die allgemeinen sprachlichen Leistungen von Kindern zu einem früheren Testzeitpunkt bilden also einen guten Prädiktor für ihre Leistungen z. B. im Rahmen einer false-belief-Aufgabe zu einem späteren Zeitpunkt. Eine umgekehrte Effektrichtung, also die Förderung von sprachlichen Fähigkeiten durch vorausgehende ToM-Kompetenzen, ließ sich nicht oder nur mit schwächerer Effektstärke nachweisen. Eine naheliegende Erklärung für diese Befunde, die als eine mögliche wenn auch schwache Hypothese in Betracht gezogen wird, lautet, dass gewisse sprachliche Grundlagenfertigkeiten zwingend notwendig sind, um ToM-Aufgaben inhaltlich zu verstehen und anschließend zu lösen. Das liegt daran, dass das übliche Testmaterial im Rahmen der verschiedenen Aufgabenstellungen der ToM-Skala (häufig kurze Bildergeschichten, gefolgt von einer Frage- und Antwortepisode zwischen Versuchsleiter und Versuchsperson) vor allem verbale Verstehens- und Lösungsstrategien erfordert. Mit diesem Erklärungsansatz ist die Relation zwischen ToM und sprachlichen Kompetenzen jedoch nicht vollständig erfasst. Um der Ursache für die gemessenen Korrelationen noch genauer auf den Grund zu gehen, wurden im Rahmen einiger der oben angesprochenen Langzeitstudien nicht nur Werte zum allgemeinen sprachlichen Entwicklungsstand der Kinder erhoben, sondern darüber hinaus die Kompetenzen innerhalb einzelner Dimensionen von Sprache (Semantik, Syntax und Grammatik) (z. B. de Villiers \& Pyers, 2002; Hale \& Tager-Flusberg, 2003). Mit Hilfe dieser Daten ist es möglich, Hinweise auf eine konstitutive Verbindung der beiden in Frage stehenden Fähigkeiten zu suchen, die deutlich über den pragmatischen Gebrauch von Sprache im Rahmen der ToM-Testsituationen hinausgeht. Die verschiedenen Subtests zum linguistischen Entwicklungsstand der Kinder deckten dabei unterschiedliche Korrelationsstärken einzelner Elemente des Sprachsystems und der ToM auf. Die Ergebnisse dieser Studien sprechen dafür, dass das lexikalische Wissen um die Bedeutungen von mental-Vokabeln wie ,denken', ,glauben', ,wissen“ etc. eine wichtige Rolle spielt, aber nicht ausreicht, um z. B. die komplexe Problematik falscher Überzeugungen verstehen zu können. Es ließ sich darüber hinaus nachweisen, dass vor allem der Leistungsstand der Kinder in den verschiedenen Tests zur Syntax von besonderer Bedeutung für die spätere Leistung beim Lösen der ToMAufgaben ist (Astington \& Jenkins, 1999; Lockl \& Schneider, 2004). Damit verlagert sich 
der Fokus des Interesses von den semantischen/lexikalischen Dimensionen, die das Verstehen und Beantworten der Testfragen betrifft, auf die strukturellen Eigenschaften von Sprache (Tager-Flusberg \& Joseph, 2005).

Doch wie lässt sich ein Zusammenhang zwischen den Kompetenzen von Kindern im Bereich sprachlicher Syntax und den gängigen ToM-Aufgaben erklären? Paradigmatische false-belief-Tests wie die Maxi- oder Smartiesaufgabe kommen mit sehr einfachen Satzgebilden aus, um das Verständnis falscher Überzeugungen von Kindern abzufragen. Diese Tests stellen entsprechend keine große Herausforderung an die Fähigkeit der Kinder, syntaktische Strukturen zu verstehen, wenn sie die Fragen des Versuchsleiters beantworten sollen. Die starken, retest-stabilen Korrelationen zwischen der Fähigkeit, falsche Überzeugungen zu verstehen und der Entwicklung linguistisch-struktureller Kompetenzen erklärt sich vielmehr mit einem genaueren Blick auf die Parallelen in den kognitiven Anforderungen der beiden Aufgabenbereiche. Der sprachliche Ausdruck von kommunikativen (X sagt, dass Y der Fall ist) und mentalen Prozessen (X denkt, dass Y der Fall ist) basiert auf einer besonderen syntaktischen Form - sogenannten Komplementsätzen. Diese Satzform besteht aus einem einleitenden und einem komplementierenden Satzteil. Gesunde Kinder, die diese spezielle syntaktische Form gebrauchen und verstehen können, bestehen in der Regel ebenfalls die verschiedenen falsebelief-Aufgaben (Hale \& Tager-Flusberg, 2003; De Villiers \& Pyers, 2002). Das Verständnis von Komplementsätzen mit ihrer besonderen Struktur scheint Kindern also die Tür zur Entschlüsselung falscher Überzeugungen zu öffnen. ${ }^{18}$ Der Grund für diese unterstützende Wirkung liegt möglicherweise in einer besonderen Eigenschaft dieser Sätze. Gemeint ist, dass der komplementierende Satzteil eine eigene, vom Wahrheitswert des gesamten Satzes unabhängige Proposition enthält. Der Satz, ,Ich denke, es regnet‘ kann durchaus wahr sein, auch wenn die Proposition, es regnet‘ im gegebenen Fall falsch ist. Dies eröffnet die Frage nach der genauen Funktion der engen kognitiven Verknüpfung zwischen Komplementsätzen und dem erfolgreichen Bestehen der verschiedenen false-belief-Aufgaben.

In die Rekonstruktion mentaler Haltung beim Entschlüsseln falscher Überzeugungen fließen zunächst Daten über den Ist-Zustand des Umfeldes, die aus dem eigenen Wissensstand gespeist werden. Hinzu kommen Informationen über den Wissensstand und Überzeugungen der beobachteten Person, die mit der Realität abgeglichen werden können. Der Beobachter

\footnotetext{
${ }^{18}$ Es soll nicht unerwähnt bleiben, dass die Korrelationen bis dato nur im Hinblick auf einen kleinen und sehr speziellen Teil des Kanons der Theory of Mind-Aufgaben (die Gruppe der false-belief Aufgaben) gemessen wurden. Der Zusammenhang syntaktischer Strukturen und anderer Aufgabentypen der ToM-Skala steht bis heute aus.
} 
muss außerdem die mentale Haltung der beobachteten Person mit dem Informationszusatz indizieren, dass sie ihre (falsche) Überzeugung für wahr hält. Würde man versuchen, den Fall einer falschen Überzeugung zu kodieren, wobei A einen Beobachter und B eine beobachtete Person darstellen (angenommen, X sei der Fall), sähe dies folgendermaßen aus: A weiß, dass X der Fall ist. A weiß, dass B denkt, Y sei der Fall. Dieses verhältnismäßig einfache Beispiel kann durch das Hinzufügen weiterer Instanzen ergänzt werden und sich damit für den Beobachter (und darüber hinaus auch eventuelle Beobachter des Beobachters) verkomplizieren. Schon kleine Änderungen erhöhen den kognitiven Schwierigkeitsgrad beträchtlich, indem sie für ihre vollständige Auflösung zusätzliche Informationen und ggf. die Dekodierung der mentalen Vorgänge eines zusätzlichen Beobachters notwendig machen. Folgende minimale Änderung verhindert beispielsweise eine vollständige Auflösung des Sachverhaltes ohne das Hinzufügen eines weiteren Elementes: $\mathrm{X}$ ist der Fall. A weiß, dass $\mathrm{X}$ der Fall ist. A denkt, dass B denkt, Y sei der Fall. Drei verschiedene Auflösungsvarianten wären an dieser Stelle denkbar. Entweder herrscht wie schon im ersten, einfacheren Fall eine falsche Überzeugung auf Seiten von B. A hat damit Recht und B denkt tatsächlich, dass Y der Fall ist. Es ist aber auch möglich, dass A nur fälschlicherweise annimmt, dass B denkt, Y sei der Fall. Damit läge der Fall der falschen Überzeugung auf Seiten von A vor und B denkt nicht, Y sei der Fall. Ebenfalls denkbar ist, dass A fälschlicherweise annimmt, dass B denkt, Y sei der Fall, denn B denkt tatsächlich, Z sei der Fall. In letzterer Auflösungsvariante liegt die falsche Überzeugung sowohl bei A als auch bei B und nur der dritte Beobachter in diesem Fall der Leser der hier dargestellten Formel mehrerer miteinander verknüpfter mentaler Einheiten - weiß, dass X tatsächlich der Fall ist und durchschaut die Situation vollständig. In allen diesen Fällen ist es wichtig, den einzelnen, Gliedern` der Formeln, die sich jeweils in einem Teilsatz sprachlich ausdrücken lassen, einen eigenen Wahrheitswert zuzuordnen. Wenn ein Mensch in der Lage ist, dies zu leisten, kann er mit Hilfe der kognitiven (syntaktischen) Verkettung von Informationen bestimmte soziale Situationen repräsentieren, interpretieren und auflösen. Ein konstruiertes Beispiel soll illustrieren, inwiefern die flexible Zuordnung von Wahrheitswerten für die Interpretation einer gegebenen Situation von entscheidender Bedeutung ist: A denkt, dass B denkt, dass C vorhat, B einen Ring zu schenken, obwohl C denkt, dass B von einem Haus träumt und daher plant, B ein Haus zu schenken. In diesem Fall sollen die unterstrichenen Elemente für diejenigen Teilsätze stehen, die von A kognitiv als ,wahr ${ }^{6}$ indiziert werden. Die kursivierten Elemente stehen für diejenigen Teilsätze, die A als ,nicht wahr' klassifiziert. Aus der Perspektive von C ist das fettgedruckte Element fälschlicherweise als ,wahr‘ indiziert, weshalb ein Missverständnis entsteht, das 
von A als solches entschlüsselt wird. Mit dieser Information ist A nun in der Lage die Enttäuschung von B, die ein Haus anstatt eines Rings geschenkt bekommt, zu antizipieren.

Die hier konstruierten Beispiele illustrieren, dass nicht nur semantische Fähigkeiten (z. B. das Wissen darum, was Vokabeln wie ,wissen`, ,denken`, ,glauben“ usw. bedeuten), sondern auch strukturell/syntaktische Fähigkeiten hilfreich bzw. sogar notwendig sind, um einige wichtige Aufgaben der ToM-Skala zu lösen. Dies betrifft vor allem diejenigen Aufgaben, in denen einer bestimmten Situation zwei miteinander konkurrierende Wahrheitswerte attribuiert werden müssen. Ein weiterer Ansatz zur Erklärung der Funktionsmechanismen der ToM kommt auf einem anderen Weg - der Untersuchung der physiologischen Grundlagen der ToM - zu einem ähnlichen Ergebnis. Auch dieser Ansatz muss an dieser Stelle Beachtung finden, um sich dem Ziel zu nähern, die kognitiven Ressourcen der ToM und ihr Funktionieren besser zu verstehen.

\subsection{ToM als kognitive Simulation?}

\section{Physiologische Grundlagen der ToM}

Bereits in der Vergangenheit wurde die restriktive Sichtweise, die menschliche Kognition sei mit einer ,Datenverarbeitungsmaschine‘ zu vergleichen, in Frage gestellt (Shapiro, 2010). Das menschliche Verhalten in sozialen Situationen erscheint in vielen Fällen unmittelbar und beinahe reflexartig zu funktionieren. Eines der leitenden Argumente gegen die Prämissen der traditionellen Kognitionswissenschaft lautet, dass die Umwandlung von Reizen aus der Umwelt in einen kognitiven Code und die darauf basierende Inferenzenbildung Zeit kostet. Das häufig spontane, scheinbar, intuitive' soziale Situationsverständnis des Menschen lässt sich mit Hilfe dieser Annahmen daher nicht zufriedenstellend erklären (Gallese, 2007, S. 659). Es muss - so neuere Annahmen - Mechanismen der unvermittelten kognitiven Interaktion zwischen dem Menschen und seiner Umwelt geben, die die entsprechenden menschlichen Handlungsmuster begründen. Solche ,direkten` Formen der Kognition werden unter dem Dach des Begriffes ,Embodied Cognition` genauer untersucht. Lawrence Shapiro schreibt über die grundsätzlichen Annahmen dieser Theorien:

In summary, the $[\ldots]$ ideas $[\ldots]$ of embodied cognition are intended to mark a shift away from thinking of the body as a mere container for the brain, or, slightly better, a contributor to the brain's activities, and toward recognizing 
the body as the brain's partner in the production of cognition. The body encorporates passive dynamics, structures information, and determines unique sensory signatures that help to create perceptual experience. (Shapiro, 2010, S. 66)

Es ist möglich, dass auch die Funktionalität der ToM mit Hilfe solcher Ansätze schlüssig beschreiben lässt. Diese Annahme wird vor allem durch die Entdeckung der sogenannten ,Spiegel-‘ Neuronen, einer spezielle Neuronengruppe im F5-Bereich des Prämotorischen Cortex, gestützt. Rizzalotti und seine Kollegen stellten im Zuge einer Studie zum Handlungsverstehen von Primaten fest, dass diese (in ihrem Fall Makaken) über eine spezielle Gruppe von Neuronen verfügen, die sowohl dann aktiv ist, wenn das Testsubjekt eine Handlung ausführt als auch dann, wenn es diese Handlung lediglich beobachtet (Rizzolatti, Fadiga, Gallese \& Fogassi, 1996). Basierend auf diesem Befund formulierten sie die Hypothese, dass diese besondere Neuronengruppe dazu dient, motorische Aktivität zu ,repräsentieren'. Spiegelneuronen bilden eine geeignete neurophysiologische Grundlage dafür, eine kognitive Verbindung zwischen beobachteten und selbst ausgeführten Handlungen herzustellen. Auch beim Menschen ist die Existenz und Funktion dieser Neuronengruppe mittlerweise gut dokumentiert. ${ }^{19}$ Das bedeutet wiederum, dass Spiegelneuronen ebenfalls bei der Dekodierung von handlungsmotivierenden mentalen Haltungen eine zentrale Rolle spielen könnten (Gallese \& Goldman, 1998). Im Zuge der genaueren Erforschung der Eigenschaften dieser Neuronengruppe wurden einige Indizien dafür, dass diese Vermutung richtig ist, sichtbar. So konnte z. B. nachgewiesen werden, dass Spiegelneuronen nicht nur dann aktiv sind, wenn ein Testsubjekt visuellen Zugang zu vollständig ausgeführten Handlungen hat. Auch wenn den Testteilnehmern unvollständige Handlungen gezeigt wurden, ließ sich die Aktivität dieser Neuronen nachweisen (Umiltà, Kohler, Gallese, Fogassi, Fadiga, Keysers \& Rizzolatti, 2001). Das bedeutet, dass Spiegelneuronen nicht allein die visuellen Eigenschaften einer Handlung repräsentieren, sondern gleichzeitig implizierte Handlungsziele ,spiegeln“. Umiltà und ihre Kollegen schreiben: „This implies that the motor representation of an action performed by others can be internally generated in the observer's premotor cortex, even when a visual description of the action is lacking“ (ebd., S. 155). Wenn Spiegelneuronen es erlauben, Handlungsziele an Hand von beobachteten Teilhandlungen zu erfassen, dann bilden sie ebenfalls eine geeignete Grundlage zur Antizipation zukünftiger ziel-

\footnotetext{
${ }^{19}$ Eine Zusammenfassung des empirischen Forschungsstandes findet sich bei Caramazza, Anzellotti, Strnad \& Lingnau (2014) und Cook, Bird, Catmur, Press \& Heyes (2014).
} 
führender Handlungsschritte und zur Herleitung von mentalen Haltungen, die mit dem entsprechenden Handlungsziel verbunden sind (Gallese, 2007, S. 662). Gleichzeitig, so vermutet Gallese, ist ein solches Handlungsverständnis mit einem ,Abgleich` eigener Erfahrungswerte im Zusammenhang mit vergleichbaren Handlungsmustern verbunden. Die Interpretation zielgerichteter, intentionaler Handlungen ist jedoch nicht der einzige Bereich der ToM, der mit Hilfe von Spiegelneuronen zu erklären ist. Auch das menschliche Verständnis fremder Emotionen lässt sich so besser verstehen. Wicker et al. konnten belegen, dass sowohl die Beobachtung typischer mimischer Äußerungen von Emotionen (in diesem Fall, Ekel`) als auch das Empfinden derselben Emotion durch die entsprechende Neuronengruppe ,gespiegelt‘ wurden. Sie folgerten aus dieser Beobachtung: „Thus, as observing hand actions activates the observer's motor representation of that action, observing an emotion activates the neural representation of that emotion. This finding provides a unifying mechanism for understanding the behaviors of others“ (Wicker, Keysers, Plailly, Royet, Gallese \& Rizzolatti, 2003, S. 655).

Mit Hilfe der neuronalen Simulation wahrgenommener Handlungen oder Emotionen (, embodied simulation mechanism ${ }^{\text {) }}$ und dem damit einhergehenden verinnerlichten Zugriff auf die Handlungen anderer können außerdem eigene Erfahrungen abgerufen werden. Menschen versetzen sich auf diese Weise, in die Schuhe“ des Handelnden (Gallese, 2007, S. 662). Gallese nennt diesen Vorgang, intentional attunement ${ }^{\star}$. Seine These lautet: „A direct form of experiential understanding of others, ,intentional attunement', is achieved by modeling their behavior as intention experiences on the basis of activation of shared neural systems underpinning what the others do and feel and what we do and feel“" (Gallese, 2006, S. 15).

\section{ToM und rekursive Syntax}

Spiegelneuronen, die sich beim Menschen u. a. im Prämotorischen Kortex und der Parietalrinde nachweisen lassen, bilden die neurophysiologische Grundlage dafür, dass wir intentionale Handlungen und Teilhandlungen repräsentieren können (Buccino et al., 2001). Wenn Teilhandlungen beobachtet werden, (z. B. eine Person A greift nach einer Tasse), die in vollständige Handlungen (z. B. A greift nach einer Tasse, führt sie zum Mund und trinkt) eingebettet sind, dann innitiiert das neuronale Aktivitätsmuster die kognitive Repräsentation der vollständigen Handlung und nicht ausschließlich der beobachteten Teilhandlung. Dadurch sind Spiegelneuronen zur Antizipation von Handlungszielen dienlich. Mit Hilfe des 
angenommenen Handlungszieles lassen sich wiederum die motivierenden mentalen Haltungen herleiten (z. B. A ist durstig und möchte deshalb etwas trinken). Die Auswahl möglicher Handlungsziele basiert laut Gallese möglicherweise auf einer Evaluation der Kontextinformationen und einer ,statistischen“ Einschätzung des wahrscheinlichsten Zieles der beobachteten Handlungen:

The statistical frequency of act sequences, as they are habitually performed or observed in the social environment, could constrain preferential paths of act inferences/predictions. This could be accomplished by chaining together different motor schemata. At the neural level, this would be equivalent to the chaining of different populations of mirror neurons coding not only the observed motor act, but also those that would normally follow in a given context. (Gallese, 2007, S. 662)

Damit sind jedoch noch nicht alle Funktionen der Spiegelneuronen erfasst. Friederici et al. konnten mittels einer fMRI-Studie nachweisen, dass phylogenetisch jüngere Bereiche des Prämotorischen Kortex (spezifisch: das Broca-Areal BA 44) dann aktiv sind, wenn hierarchisch strukturierte (rekursive) Satzgebilde prozessiert werden. Im Gegensatz dazu werden einfache Satzgebilde hingegen auch in älteren Arealen des Gehirns, die wir mit Primaten teilen, prozessiert. Die Eigenschaft der Neuronen des Präfrontalen Kortex, die kognitive Repräsentation von Sequenzen und eingebettete Strukturen initiieren zu können, bildet aus evolutionärer Sicht den Grundstein für ein elaboriertes Kommunikationssystem, mit dessen Hilfe nicht nur einfache Sätze, sondern auch komplexe Satzgefüge (z. B. Sätze mit eingebetteten Satzteilen) kognitiv ausgewertet werden können (Gallese, 2007, S. 666; Hauser, Chomsky \& Fitch, 2002, S. 1578). Hauser et al. stellen aus diesem Grund die Hypothese auf, dass es sich bei der Fähigkeit, rekursive syntaktische Sätze zu bilden und zu verstehen, um das zentrale Alleinstellungsmerkmal der menschlichen Spezies hinsichtlich des Sprachvermögens handelt. Während Primaten dazu in der Lage sind, einfache Satzsequenzen zu erlernen und zu verstehen, gelingt es ihnen - im Gegensatz zum Menschen - nicht, rekursive Strukturen kognitiv zu erfassen. Rekursive Sätze zeichnen sich dabei nicht nur durch eine Struktur der Einbettung von untergeordneten Satzteilen in übergeordnete Satzteile aus. Um solche Sätze verstehen zu können, ist es ebenfalls wichtig, die semantische Relation, in denen die eingebetteten Satzteile sich auf die übergeordneten Satzteile beziehen, zu bewerten. Ein solches Verständnis ist ein entscheidender Bestandteil des menschlichen Sprachvermögens und gestattet uns, Sätze wie [Der Junge, (den das Mädchen ansah), war groß.] zu bilden und zu verstehen (Frederici, Bahlmann, Heim, Schubotz \& Anwander, 2006). Dieser Satz enthält im Ganzen zwei Subjekte - einen Jungen und ein Mädchen. Dank der Fähigkeit die 
beiden Satzteile entsprechend den grammatischen Markern (hier z. B. anhand der Verwendung der grammatischen Fälle) zu interpretieren, können wir problemlos verstehen, wer laut dieser Aussage wen ansieht und wen von beiden das Adjektiv ,groß‘ betrifft. Das Hirnareal, das mit diesen Fähigkeiten in Verbindung gebracht werden konnte ist das Broca-Areal:

[...] Broca's area, due to its premotor origin, is involved in the assembly of meaningless sequence of action units (whether finger or phonoarticulatory movements) into meaningful representations. This elaboration process may proceed in two directions. In production, Broca's area recruits movement units to generate words/hand actions. In perception, Broca's area [...] addresses the vocabulary of speech/hand actions, which form the template for action recognition. Our hypothesis is that, in origin, Broca's area precursor was involved in generating/extracting action meanings by organizing/interpreting motor sequences in terms of goal. Subsequently, this ability might have been generalized during the evolution that gave this area the capability to deal with meanings (and rules), which share similar hierarchical and sequential structures with the motor system. (Fadiga at al., 2006, S. 87)

Die Fähigkeit, rekursive Satzstrukturen zu bilden und zu verstehen, führt dazu, dass wir mittels einer begrenzten Anzahl bedeutungstragender Zeichen eine potenziell unendliche Menge unterschiedlicher komplexer Strukturen erstellen können, die sich in ihrer Bedeutung systematisch voneinander unterscheiden. Chomsky schreibt: „Human language is based on an elementary property that also seems to be biologically isolated: the property of discrete infinity [...]“ (Chomsky, 2000, S. 3). Damit ist jedoch eine theoretische (potenzielle) Unendlichkeit gemeint, deren Realisierung im Rahmen tatsächlicher sprachlicher Äußerungen durch allgemeine kognitive Verarbeitungskapazitäten begrenzt wird.

Die Ergebnisse der überwiegend empirischen Studien, die in diesem Abschnitt genannt wurden, legen den Schluss nahe, dass bestimmte Bereiche des Premotorischen Kortex uns dazu befähigen, Handlungssequenzen zu strukturieren. Dies geschieht mit Hilfe von generierten und abrufbaren Aktivitätsmustern von Spiegelneuronen. Wird ein solches Muster ,erkannt‘, ist es wiederum möglich, passende Handlungsziele und motivierende mentale Haltungen zu assoziieren. Gleichzeitig weisen die Ergebnisse einiger bildgebender Studien darauf hin, dass dieses spezifische Neuronensystem gleichzeitig die Grundlage dafür ist, dass Menschen rekursive syntaktische Strukturen bilden und verstehen können. Dies gestattete es dem Menschen im Zuge der Evolution ein sehr viel komplexeres Kommunikationssystem auszubilden, als seine nächsten Verwandten - die Primaten. Die Fähigkeit, komplexe miteinander verkettete mentale Haltungen in einem sozialen Kontext zu dekodieren und für ein soziales 
Handlungsverständnis zu nutzen, ist ebenfalls ein evolutionäres Alleinstellungsmerkmal des Menschen und zeichnet sich ebenfalls durch rekursive kognitive Strukturen aus. Dies führt zu der Überlegung, dass zwei unterschiedliche soziale Kompetenzen des Menschen - die menschliche Sprache und die elaborierte ToM gesunder Erwachsener - zumindest zum Teil auf denselben neuronalen Mechanismen beruhen. Das ist ein weiteres Indiz im Sinne der in einem früheren Abschnitt dieser Arbeit referierten empirischen Ergebnisse, die auf einen Zusammenhang zwischen der ToM und den menschlichen Fähigkeiten, syntaktische Satzkonstruktionen zu entschlüsseln, hindeuten. Die Rekursivität der menschlichen Syntax findet hier ein strukturell analoges Äquivalent in der Rekursivität der ToM gesunder Erwachsener. Sie ist u. a. dadurch gekennzeichnet, dass ein Verstehen selbstbezüglicher oder eingebetteter mentaler Haltungen (,Ich denke, dass ich denke' Oder: ,Ich denke, dass Du denkst, dass ich möchte, dass... ' usw.) bis zu einem gewissen Grad der Komplexität problemlos möglich ist (Liddle \& Nettle, 2006). Daher liegt die Vermutung nahe, dass aufgrund der strukturellen Parallelen der involvierten kognitiven Verstehensprozesse dieselben Mechanismen zum Einsatz kommen können. Auf physiologischer Ebene sind Spiegelneuronen eine wahrscheinliche kognitive Ressource, die diese Prozesse ermöglichen und lenken.

\subsection{Narrative Strukturen des psychologischen Handlungsverständnisses}

Wie die ToM tatsächlich funktioniert, lässt sich nicht nur auf Basis physiologischer Grundlagen, sondern mit Hilfe einer funktionalen Beschreibung der relevanten kognitiven Prozesse erfassen. Eine weitere Überlegung zur Erklärung der ToM lautet, dass die Organisation der sie ermöglichenden kognitiven Prozesse und Mechanismen den Prinzipien einer narrativen Strukturierung folgt. Der Ursprung dieses Ansatzes lässt sich zu den Arbeiten von Jerome Bruner zurückverfolgen, der auf eine grundlegende Dichotomie des menschlichen Denkens hinweist. Ihm zufolge lassen sich zwei ,Modi` des Denkens unterscheiden: der logisch-paradigmatische Modus einerseits und der narrative Modus andererseits. Beide Modi dienen dazu, Schlüsse zu ziehen und auf diesem Weg Wissen und Erkenntnisse zu gewinnen. Sie unterliegen jedoch jeweils unterschiedlichen Funktionsprinzipien. Ersterer richtet sich laut Bruner nach den formalen Gesetzmäßigkeiten des Erklärens. In diesem Fall werden Informationen kategorisiert und übergeordneten Konzepten zugeordnet. Das Instrumentarium zur Herstellung von sinnstiftenden Verknüpfungen besteht in diesem Fall aus Konjunktionen, Disjunktionen, Hypero- und Hyponymien, Folgebeziehungen u. Ä. (Bruner, 1986, 
S. 11). Dem steht der narrative Modus des Denkens gegenüber. Dieser narrative kognitive Modus manifestiert sich in kognitiven narrativen Artefakten wie z. B. Geschichten. Weil die Struktur des menschlichen Denkens Eigenschaften aufweist, die wir als ,narrativ` beschreiben können, produzieren wir Geschichten, in denen sich diese Eigenschaften widerspiegeln. Wenn diese Annahme richtig ist, sollten sich an Hand dieser Artefakte im Umkehrschluss die Eigenschaften und Strukturen dieses kognitiven Denkmodus ,ablesen` lassen. Aufgrund ihrer inhärenten Eigenschaften unterstützen narrative Strukturen das kognitive Problemlöseverhalten des Menschen entscheidend. Herman benennt insgesamt fünf narrative Eigenschaften in diesem Zusammenhang: 1. Handlungen und Erfahrungen sind in der Regel in leicht zu prozessierende Episoden unterteilt. Jede Geschichte besteht aus einer Sequenz von Ereignissen, die zumeist auf den Handlungen und Empfindungen von Figuren beruhen. 2. die einzelnen Episoden sind durch implizite oder explizite kausale Relationen miteinander verknüpft. 3. die dargestellten Handlungen lassen sich mit gegebenen kulturellen Erwartungshaltungen kontrastieren. 4. Handlungen werden im Kontext von typischen bzw. häufigen Handlungsmustern dargestellt. 5. Handlungen und Ereignisse können aus mehr als einer einzigen Perspektive präsentiert werden. Um eine Geschichte verstehen zu können, muss ein Interpret dazu in der Lage sein, die einzelnen Elemente im Rahmen der gesamten vermittelten Struktur und vor dem Hintergrund aller gegebenen semantischen Informationen zu erfassen. Handlungen können dabei entweder auf typische Handlungsmuster zurückgeführt werden oder müssen im Kontext der im Einzelfall gegebenen Informationen entschlüsselt werden. David Herman (2013, S. 294) präzisiert diesen Gedanken:

[...] narrative furnishes a powerful technology for building models of action sequences. Such models enable storytellers and story-interpreters to assess the motivations, structure, and consequences of actions by varying perspectival and attitudinal stances toward those actions and the situations in which they occur. Narrative can also be used to manipulate time-scales so as to compress or elongate chains of events, and to reorder events in ways that allow for targeted assessments of particularly salient links within those chains; to cluster together - or ,emplot' - discrete behaviors into goal-directed patterns of action, $[\ldots]$ and to generate and cross-compare counterfactual scenarios that allow the domain of the actual to be profiled against a larger universe of possibilities $[\ldots]$.

Die genannten Eigenschaften lassen darauf schließen, dass Geschichten eine geeignete Grundlage zur Vermittlung interpretativer Strategien und Techniken bilden, die es dem Menschen erlauben, typische und spezifische soziale Situationen adäquat verstehen zu können 
(Bruner, 1990, S. 47). Es ist durchaus denkbar, dass sich die vermittelten Handlungsmuster und ihr Zusammenhang mit explizit oder implizit vermittelten Informationen zu bestimmten mentalen Haltungen kognitiv in ,narrativer ${ }^{6}$ Form gespeichert werden können. Diese Überlegungen führen Daniel D. Hutto (2009) zu der Formulierung der ,Narrative Practice Hypothesis‘ (NPH). Sie besagt, dass Kinder die ToM-Fähigkeiten in der Konfrontation mit (erzählten) Geschichten erlernen. Dies stünde im Gegensatz zu der bereits zuvor beschriebenen Hypothese von Leslie, der von einem Reifungsprozess des ToM-Mechanismus ausgeht und der auch ohne einen Lernstimulus stattfindet. Hutto dahingegen schreibt: „Children are not FP [Folk Psychology] competent until they have mastered certain narrative skills. Engaging in narrative practice is the source of our FP understanding“ (ebd. S. 13). ${ }^{20}$ Hierfür sind laut Hutto jedoch Geschichten erforderlich, die die mentalen Vorgänge fiktiver Charaktere und ihre Auswirkungen auf die entsprechenden Handlungen explizit thematisieren oder besonders hervorheben (ebd., S. 11). Solche Geschichten, die Hutto folk psychological narratives nennt, geben Kindern Einblicke in den Zusammenhang zwischen mentalen Vorgängen, Personen und Handlungen. Durch die Konfrontation mit solchen Texten eignen sich Kinder die für die ToM entscheidenden kognitiven Strategien zum Verständnis ihrer eigenen Umwelt an. Diese Annahme wurde in der jüngeren Vergangenheit durch die empirische Beobachtung gestützt, dass Kinder in demselben zeitlichen Entwicklungsabschnitt Fortschritte hinsichtlich ihrer narrativen als auch hinsichtlich ihrer ToM-Kompetenzen erzielen (ebd., S. 22). Mit anderen Worten, Kinder entwickeln in einem Altersabschnitt von 3-5 Jahren nicht nur die zentralen Fähigkeiten, die der ToM zuzuschreiben sind, sondern beginnen auch dann, eigenständig und zusammenhängend von (fiktiven und realen) Ereignissen zu erzählen. Diese Beobachtung ist allerdings kein zufriedenstellender Beleg für eine Verbindung der beiden Kompetenzbereiche, da sich beide ebenfalls den gleichen Entwicklungszeitrahmen mit weiteren kognitiven Fähigkeiten (Sprache, Exekutive Funktionen etc.) teilen. Außerdem ist nicht weiter erschlossen, ob sich die Fähigkeiten der ToM aufgrund der Konfrontation mit narrativen Kontexten erlernen oder weiterbilden lässt, oder ob wir erst dank derselben Fähigkeit zur Rezeption von Geschichten mit einer psychologischen Handlungsmotivierung in der Lage sind. Allerdings lässt sich nicht leugnen, dass Geschichten - und damit auch literarische Erzähltexte - einerseits Produkte des menschlichen Geistes und andererseits Gegenstand kognitiver Interpretationsprozesse sind. Der Gedanke, dass sich in ihnen die Struk-

\footnotetext{
${ }^{20}$ Kursiviert mein Einschub zur Erklärung des von Hutto verwendeten Akronyms. Der Terminus ,Folk Psychology', bezeichnet hier wie der Begriff der , Theory of Mind` die menschliche Fähigkeit mentale Haltungen und innere Vorgänge nachzuvollziehen.
} 
turen des menschlichen Denkens manifestieren und sie daher - bei einer genauen Untersuchung - ebenfalls über sie Auskunft geben können, ist vor allem in methodologischer Hinsicht für den dritten Teil dieser Arbeit, der der Analyse konkreter Textbeispiele gewidmet ist, interessant.

\section{$5 \quad$ Fazit - Was ist ToM und wie funktioniert sie?}

Die Interpretations- und Verstehensleistungen des Menschen in einem sozialen Umfeld sind vielfältig. Die Attribution und Deutung mentaler Haltungen, innerer Vorgänge und deren Bezug zu sozialen Handlungen und Verhaltensweisen spielen dabei in zahlreichen Bereichen der zwischenmenschlichen Interaktion und der Selbstreflexion eine wichtige Rolle. Mit einem Blick auf den empirischen und theoretischen Forschungsdiskurs der kognitiven Entwicklungspsychologie ist in den vorangehenden Abschnitten deutlich geworden, dass die ToM einen ganzen Katalog unterschiedlicher sozialer Verstehensleistungen betrifft und eine Vielzahl kognitiver Fähigkeiten umfasst. Sie ist ein zentrales Element der menschlichen Kognition, ohne welches eine Vielzahl typischer sozialer Interaktionsmuster des menschlichen Alltags nicht zu bewältigen wäre. Unsere ToM erlaubt es uns, in vielen unterschiedlichen Situationen, die Handlungen anderer zu interpretieren oder zu antizipieren, auch wenn diese mit den von uns als real angenommenen Umständen in Konflikt stehen. Es wurde deutlich, dass diese Fähigkeit für das Verständnis unterschiedlicher Situationen der sozialen Interaktion hilfreich und für manche (z. B. die Synchronisation von Wahrnehmung, das Fiktionsspiel, das Verstehen falscher Überzeugungen, das Verstehen uneigentlicher Kommunikation usw.) sogar eine Voraussetzung ist. Es wurde deutlich, dass eine Reihe von unterschiedlichen kognitiven Mechanismen (IK, Arbeitsgedächtnis, ,Entkopplungsmechanismus' etc.) für die Verstehensleistungen gesunder Erwachsener im Zuge unterschiedlicher ToM-relevanter Aufgaben verantwortlich sind. Daher scheint es unwahrscheinlich, dass sich das gesamte Spekturm der kognitiven Leistungen, die ein Mensch erbringen muss, um diese Aufgaben zu bewältigen, auf einen einzelnen, spezifischen kognitiven ,ToM-Mechanismus ${ }^{\star}$ zurückführen lässt. Vielmehr ist es wahrscheinlich, dass es sich um ein System verschiedener ineinandergreifender kognitiver Funktionen handelt, welches den verschiedenen Leistungen zugrunde liegt. Die ToM ist also - allgemein gesprochen - eine von vielen kognitiven Fähigkeiten des Menschen. Sie betrifft die Wahrnehmung und das Verstehen mentaler und psychologischer Vorgänge und wird durch die Gesamtheit und das Zusammenspiel einer 
Reihe kognitiver Mechanismen gewährleistet. Einige dieser kognitiven Mechanismen - wie zum Beispiel das konzeptuelle Wissen um die Bedeutung und Funktion von verschiedenen mentalen Haltungen (,Überzeugung'vs. ,Wissen‘, vs. ,Wunsch') scheinen dabei spezifisch der ToM zu dienen. Es sind darüber hinaus aber auch eine Reihe allgemeiner kognitiver Ressourcen (das Arbeitsgedächtnis, die Inhibitorische Kontrolle, semantische und syntaktische kognitive Kompetenzen etc.) involviert, die zugleich auch andere menschliche Fähigkeiten tragen.

Ziel dieses ersten Arbeitskapitels war es, die verschiedenen sozialen und kognitiven Fähigkeiten des Menschen vorzustellen, die mit der ToM assoziiert sind. Die überwiegend empirisch und mit Hilfe von Metastudien gewonnenen Erkenntnisse aus der kognitiven Entwicklungspsychologie über die soziale Funktion und Entwicklung der ToM im Kindesalter gewährten einen Einblick in die sozialen Kompetenzen, die durch die Fähigkeit, mentale Haltungen und Vorgänge zu attribuieren, möglich sind. Daran anschließend stellte sich die Frage nach den kognitiven Mechanismen, die uns Menschen diese besonderen Fähigkeiten ermöglichen. Dabei standen zunächst die epistemologischen Grundlagen der Verarbeitung unbeobachtbarer, innerer Vorgänge im Fokus theoretischer kognitionswissenschaftlicher Überlegungen. Die wichtigsten in diesem Kontext besprochenen Ansätze sind die Theorie der ,Meta'-kognition, die ,Theorie-Theorie‘, die ,Simulationstheorie‘ und die ,NarrativitätsTheorie ${ }^{6}$, deren Ziel es ist, zu erklären, welche kognitiven Prozesse und Mechanismen der ToM zugrunde liegen. Darüber hinaus stand die Frage im Fokus, ob der ToM spezifische hirnphysiologische Mechanismen (z. B. Spiegelneuronen oder ein ,Entkopplungsmechanismus') zugrunde liegen oder ob sich durch die Ausbildung allgemeiner kognitiver Fähigkeiten (z. B. die Fähigkeit zur Bildung mentaler Modelle, die Ausbildung des Arbeitsgedächtnisses, der Inhibitorischen Kontrolle oder sprachlicher Fähigkeiten) erklären lässt.

Der Überblick über die einzelnen Ansätze lässt ein Problem erkennen: Die Hypothesen, die im Rahmen der verschiedenen Forschungsdiskurse zur Funktionalität der ToM formuliert werden, beziehen sich auf unterschiedliche Beschreibungsebenen der menschlichen Kognition. Diese Ebenen umfassen 1. hirnphysiologische Mechanismen (z. B. Spiegelneuronen), 2. funktional benannte ToM-spezifische kognitive Mechanismen (z. B. der ,Entkopplungsmechanismus ${ }^{\circ}$, 3. allgemeine Prinzipien der menschlichen Kognition, die allen kognitiven Prozessen und damit auch der ToM zugrunde liegen (z. B. die Repräsentation von Informationen mittels eines kognitiven ,Codes ${ }^{`}$ und mentaler Modelle), 4. allgemeine kognitive Mechanismen der Informationsverarbeitung, die die Funktionalität der ToM unterstützen (das 
Arbeitsgedächtnis, die Inhibitorische Kontrolle, lexikalische und syntaktische Kompetenzen), 5. die Beschreibung von heuristischen Strategien zur Wahrnehmung und Evaluation von situationsrelevanten mentalen Haltungen (die Fähigkeit zur ,Simulation“ fremder mentaler Vorgänge mit Hilfe der eigenen; die Fähigkeit, mentale Kontexte mit Hilfe einer ,naiven Theorie‘ - also mit Hilfe von abstraktem Wissen über die Funktion und Bedeutung von mentalen Haltungen - erschließen zu können; mittels metakognitiver Fähigkeiten) und zuletzt 6. die qualitative Beschreibung von Denkprozessen zur kognitiven Situationsrekonstruktion (z. B. mittels ,narrativer' Informationsstrukturierung). Keiner dieser Ansätze ist allein dazu geeignet, die Funktionsweise der ToM in ihrer gesamten Bandbreite zufriedenstellend zu erklären. Auf Grund der unterschiedlichen Beschreibungsebenen lassen sich diese Ansätze im Sinne einer umfassenden Definition des ToM-Begriffes allerdings auch schwer miteinander vergleichen, um zum Beispiel für den Zweck der vorliegenden Untersuchung eine grundlegende Arbeitsdefinition zu entwickeln. Allerdings ist es möglich, einzelne Mechanismen der ToM funktional zu beschreiben, wenn man die verschiedenen sichtund messbaren Leistungen gesunder Erwachsener auf die funktional dafür notwendigen kognitiven Mechanismen zurückführt. Es liegt dementsprechend nahe anzunehmen, dass die Fähigkeit, die uns als ,ToM` bekannt ist, durch das (möglicherweise situationsabhängige) Zusammenspiel simulativer, abstrahierender, metakognitiver und integrativer Kognitionsprozesse ermöglicht wird.

Die wichtigsten Leistungen, die Menschen im Zuge der verschiedenen ToM-Aufgaben erbringen müssen scheinen mir die folgenden zu sein: 1. Die Fähigkeit, Funktionen und Inhalte von unterschiedlichen Kategorien mentaler Haltungen (Überzeugung vs. Wissen vs. Wunsch etc.) in Relation zueinander zu setzen. 2. Die Fähigkeit zur Kohärenzgenese. 3. Die Koordination und Lenkung der eigenen und fremden Aufmerksamkeit. 4. Das Verstehen intentionaler, motivierter Handlungen. 5. Die Fähigkeit zur Perspektivübernahme und Situationsrekonstruktion und -repräsentation aus eigener und fremder Perspektive. 6. Die Rekonstruktion der räumlichen und persönlichen Relationen zwischen Personen und Objekten. 7. Sprachproduktion und -verstehen, die Sequenzierung komplexer Informationsstrukturen und damit die Fähigkeit zum Verstehen syntaktischer Einbettungen. 8. Damit geht die Fähigkeit zur Sequenzierung und Einbettung komplexer Informationsgruppen einher (Rekursivität und ,Entkopplung'). 9. Die Assoziation und Synthese von weit auseinanderliegenden Informationsgruppen und das episodische Gedächtnis. 10. Die Unterdrückung salienter Stimuli aus der Umwelt, wie z. B. das eigene Wissen um einen tatsächlichen Sachverhalt. 
Allen weiteren Überlegungen in dieser Arbeit liegt also die Annahme zugrunde, dass der Begriff der ,ToM' lediglich ein Überbegriff ist. Er bezeichnet auf einer allgemeinen Ebene die Fähigkeit des Menschen, anderen mentale Haltungen und Vorgänge zuzuschreiben fremde Handlungen darauf basierend zu interpretieren bzw. die eigenen Handlungen daran auszurichten. Um die Funktion der ToM im Zuge der literarischen Rezeption im Detail zu verstehen, ist es allerdings sinnvoll, in Zukunft den grundlegenden kognitiven Mechanismen Beachtung zu schenken, die in diesem Kapitel zusammengetragen wurden. Nur so kann die Rolle dieser besonderen menschlichen Fähigkeit für das Verstehen und Interpretieren literarischer Erzähltexte genauer, als es bisher geschehen ist, aufgeschlüsselt werden. 


\section{Die Rolle der ToM in der literarischen Rezeption}

\section{$1 \quad$ Einleitendes}

Die Frage lautet nun allgemein formuliert: In welcher Form sind die Mechanismen der ToM in die literarische Rezeption eingebunden bzw. welche Rolle spielen sie grundsätzlich für die literarische Rezeption? Im folgenden Kapitel möchte ich mich dieser Frage zuwenden und im Zuge meiner Argumentation auf die Hypothesen von Lisa Zunshine, Alan Palmer und anderen aufbauen. Sie argumentierten, dass Leser ihre ToM dazu nutzen, um fiktiven Figuren mentale Haltungen und Vorgänge zuzuschreiben. Außerdem antizipieren die Autoren literarischer Texte die Attributionsvorgänge ihrer Leser ebenfalls mit Hilfe ihrer eigenen ToM und können die relevanten Einsichten während der Gestaltung von Inhalt und Struktur ihrer Texte berücksichtigen. Ich möchte herausfinden, ob sich die kognitiven Leistungen, die mit diesen Vorgängen zusammenhängen, noch präziser, als es bisher geschehen ist, erfassen lassen. Gegebenenfalls lassen sich weitere Funktionen der ToM, als diejenigen, die bisher benannt wurden, identifizieren. Darüber hinaus gilt es die methodischen Möglichkeiten der hier zur Verfügung stehenden Mittel für eine solche Aufgabenstellung kritisch zu reflektieren.

Die eingangs formulierte übergeordnete Fragestellung wird im Folgenden aus zwei unterschiedlichen Perspektiven heraus beleuchtet. Die erste methodische Perspektive ist der Kategorie Hypothesen zur Relation zwischen Textmerkmal und Leserkognition zuzuordnen. Dabei gilt die Aufmerksamkeit den strukturellen Gemeinsamkeiten zwischen zwei auf den ersten Blick unterschiedlichen kognitiven Fähigkeiten bzw. Leistungen (der ToM auf der einen und der Rezeption literarischer Erzähltexte auf der anderen Seite). Ziel ist es, diejenigen grundlegenden strukturellen Gemeinsamkeiten zwischen textuellen und kognitiven Strukturen zu identifizieren, die einerseits den Schluss rechtfertigen, dass die ToM im Zuge 
der Rezeption literarischer Texte ebenso eingesetzt werden kann, wie im Zuge der Interpretation realer Situationen. Allerdings möchte ich auch nicht aus dem Blick verlieren, dass von der intuitiven Annahme, Leser interpretieren literarisch geschilderte Handlungen in vergleichbarerer Weise mit realen sozialen Handlungen einmal abgesehen - es aus theoretischer Sicht ebenfalls denkbar ist, dass literarische Texte aufgrund ihrer besonderen Beschaffenheit abweichende Bedingungen für die Informationsverarbeitung an ihre Leser stellen. Neben grundlegenden Überlegungen zur Rolle der ToM in der literarischen Rezeption möchte ich vor allem eine genauere Vorstellung darüber gewinnen, auf welche die kognitiven Mechanismen der ToM im Zuge der Lektüre eines Erzähltextes zum Einsatz kommt. Die Untersuchungen zur Relation zwischen Kognition und Textbeschaffenheit sind eine wichtige Voraussetzung, um sich dieser zweiten methodischen Perspektive - also der Hypothese, die ich einleitend ,Vorraussetzungs '-Hypothese genannt habe - zuzuwenden. Für beide Untersuchungen soll ebenfalls reflektiert werden, ob sich die entsprechenden Fragestellungen im Rahmen einer theoretischen Arbeit zufriedenstellend beantworten lassen.

Ich stelle allen nachfolgenden Überlegungen die Annahme voran, dass der Einsatz und das Zusammenspiel von kognitiven Mechanismen und Ressourcen im Einzelfall von der gegebenen Informationslage, den Dispositionen des individuellen Beobachters/Interpreten und den Verstehensanforderungen der einzelnen Situation abhängt. Um die in dieser Arbeit zentrale Frage zur Rolle der ToM im Rahmen der kognitiven Textrezeption beantworten zu können, ist ein Vergleich der beiden Interpretationskontexte und ihrer Kognitionsanforderungen notwendig. Ich betrachte dabei sowohl die reale soziale Umgebung des Menschen als auch literarische narrative Texte jeweils als Summe von Informationen, die in bestimmter Form strukturiert sind und in bestimmter Form an einen Interpreten vermittelt werden. Sowohl im realen sozialen Kontext als auch im Zuge der Lektüre eines literarischen Erzähltextes müssen die gegebenen Informationen basierend auf einer Reihe vorhandener (oder fehlender) Vorinformationen mit Hilfe einer Interpretations- oder Verstehensleistung ausgewertet werden. Mein Interesse gilt den online-Kognitionsprozessen, die während der Lektüre eines typischen literarischen Erzähltextes stattfinden und die einen Bezug zur ToM besitzen. Diese Prozesse, die im Folgenden synonym auch als ,Rezeptions'- und ,Interpretationsprozesse“ bezeichnet werden, betreffen den kognitiven Vorgang, der während der Lektüre eines literarischen Textes abläuft. Ausgehend von der Annahme, dass die während der Lektüre ablaufenden Verstehensprozesse einerseits von der allgemeinen kognitiven Disposition des Menschen bestimmt und andererseits auf Grund der Textkonfiguration auf bestimmte Art 
und Weise stimuliert werden, möchte ich die Art und Weise sowie die absehbaren Konsequenzen dieser Stimulation mit einem Blick auf die Textkonfiguration näher bestimmen. Um die allgemeinen und spezifischen Merkmale literarischer Erzähltexte zu erfassen, sind bestehende Konzepte und Begriffe aus der literaturtheoretischen Forschung hilfreich. Sie geben Auskunft über die typischen Charakteristika von literarischen Texten und ermöglichen es, ihre spezifischen Merkmale genau zu beschreiben. Auf der anderen Seite dienen die im ersten Kapitel vorgestellten kognitiven Leistungen, Mechanismen und Ressourcen, die es dem Menschen ermöglichen, eine ToM zu haben', als zentrale Anhaltspunkte für die Analyse der Kognitionsanforderungen, die literarische Texte an ihre Leser stellen. Darauf aufbauend lassen sich die kognitiven Anforderungen eines typischen literarischen Erzähltextes bestimmen. Ich möchte prüfen, ob sich bestimmen lässt, dass bestimmte kognitive Mechanismen der ToM für die Lektüre eines (typischen oder spezifischen) literarischen Erzähltextes vorausgesetzt, besonders gefordert oder gezielt blockiert sind. Die Gliederung des nachfolgenden zweiten Hauptkapitels orientiert sich an den Aufgabenstellungen, die sich einem Leser stellen, der einen literarischen Erzähltext zur Hand nimmt und zu lesen beginnt. Zunächst gilt es die Liste dieser Aufgabenstellungen einzugrenzen und für den Rahmen dieser Arbeit zu bestimmen. Die auf diesem Weg identifizierten ,Eckpfeiler` der kognitiven Rezeption bilden den Rahmen für die Suche nach Bezugspunkten mit den bereits im ersten Teil der Arbeit vorgestellten kognitiven Mechanismen der ToM.

Ich habe bereits an einer vorangehenden Stelle erwähnt, dass es schwierig ist, im Rahmen einer theoretischen Arbeit eine Antwort auf diese Frage zu suchen und gleichzeitig die individuellen Dispositionen empirischer Leser im Auge zu behalten. Ich möchte daher im Folgenden keine Rücksicht auf die Voraussetzungen nehmen, die ein bestimmter realer Leser mit sich bringen könnte. Wenn innerhalb der folgenden Argumentation von einem Leser oder Rezipienten die Rede ist, ist daher immer das bereits einleitend beschriebene Konzept eines Modell-Lesers gemeint. Die Funktion eines Modell-Lesers soll darin bestehen, diejenigen kognitiven Prozesse auszuführen, die durch bestimmte semantische oder strukturelle Eigenschaften eines Textes im Sinne der Kohärenzgenese erforderlich oder möglich sind. Ein solches hypothetisches Leser-Konstrukt erlaubt es, einen systematischen Überblick über alle diejenigen Kognitionsprozesse zu erlangen, die typischerweise in die Rezeption eines literarischen Erzähltextes eingebunden sind. Darüber hinaus können Einblicke in die Relationen zwischen textuellen Merkmalen und kognitiven Prozessen gewonnen werden. Unter der hypothetischen Voraussetzung, dass alle unabhängigen Variablen (z. B. die gegebenen 
kognitiven Dispositionen des Lesers) unveränderlich sind, wird es möglich, Hypothesen über die Wirkung typischer Merkmale literarischer Erzähltexte zu formulieren.

\section{Initiation eines geeigneten Rezeptionsmodus}

Welche Aufgaben stellen sich einem Leser, wenn er einen literarischen Erzähltext zur Hand nimmt und zu lesen beginnt? Zunächst einmal muss er eine geeignete kognitive Rezeptionshaltung dem Text gegenüber einnehmen. Diese Rezeptionshaltung ist vor allem aufgrund einer zentralen Eigenschaft vieler literarischer Erzähltexte erforderlich. Gemeint ist die Fiktionalität des dargestellten Geschehens. Mit anderen Worten: Wenn ein Leser einen literarischen Erzähltext zur Hand nimmt und zu lesen beginnt, dann muss er - um ihn angemessen rezipieren zu können - anerkennen, dass das dargestellte Geschehen in einem besonderen Verhältnis zur Realität steht. ${ }^{21}$ Das bedeutet, dass die Fähigkeit, zwischen Realität und Fiktion unterscheiden zu können, ein wichtiger Aspekt der Rezeption literarischer Erzähltexte ist, der für ihre angemessene Rezeption erforderlich ist. Die Entwicklung dieser Fähigkeit lässt sich, wie im ersten Teil dieser Arbeit näher beschrieben, bei jungen Kindern im Rahmen ihres Fiktionsspiels beobachten. Racoczy schreibt: „Human fictional activities have their roots in the emergence of pretend play in the 2nd year of life“ (Rakoczy, 2008, S. 1195). Wie Rakoczy gehe auch ich davon aus, dass die kognitiven Fähigkeiten, die sich zu diesem Zeitpunkt der Entwicklung zum ersten Mal zeigen, ein zentrales Prärequisit zum späteren adäquaten Umgang mit fiktionaler Literatur sind.

Die Frage wie und warum wir Menschen zur Unterscheidung von Fiktion und Realität in der Lage sind bzw. wie die Kognition der Fiktionswahrnehmung genau funktioniert, ist bisher noch nicht abschließend geklärt worden. Um herausfinden zu können, welche kognitiven Mechanismen uns die Fiktionsrezeption in der Form, in der wir sie gewohnt sind, gestatten, ist es sinnvoll, literaturwissenschaftliche Fiktionstheorien zu Rate zu ziehen. Allerdings stellt sich hier die Aufgabe, unter den zahlreichen Ansätzen der unterschiedlichen fiktionstheoretisch ausgerichteten Diskussionen diejenigen zu bestimmen, die für die hier gestellte

\footnotetext{
${ }^{21}$ Selbstverständlich gibt es in realen Fällen der Rezeption, in denen die Fiktionalität eines Textes fälschlicherweise nicht erkannt, oder einem Text fälschlicherweise die Eigenschaft, fiktional zu sein, zugeschrieben wird. Ich möchte solche Fälle gegenwärtig aus der Untersuchung ausschließen, um zunächst die hier gestellten grundsätzlichen Fragen zu klären. Allerdings sei anerkannt, dass es sich bei solchen Fällen um besonders interessante Untersuchungsgegenstände aus kognitiv-literaturwissenschaftlicher Perspektive handelt, bei denen nach Gründen für eine solche Fehleinschätzung seitens des Lesers gesucht werden kann. Dies soll zukünftigen Arbeiten vorbehalten bleiben.
} 
Forschungsfrage hilfreich sind. Das liegt unter anderem daran, dass der Diskurs um das umstrittene Phänomen der Fiktionalität aus unterschiedlichen Perspektiven, mit unterschiedlichen Zielen und daher auch unter unterschiedlichen methodischen Voraussetzungen geführt wird. So gibt es zahlreiche Beiträge, die eine ontologische Bestimmung des Begriffes ,Fiktionalität‘ anstreben, aber auch solche Beiträge, die eine sprachphilosophische oder kognitive Herangehensweise wählen und so jeweils andere Aspekte des Phänomens in den Vordergrund stellen. Wo also soll die Suche nach den Relationen zwischen den Merkmalen eines Textes, die durch seine Fiktionalität begründet sind, und den relevanten kognitiven Verarbeitungsprozessen sinnvollerweise beginnen? Ausgangspunkt ist hier Racoczys Beschreibung von kindlichen Fiktionsspielen. Er schreibt: ,Joint pretence games are implicit rule-governed activities with a normative structure: Given shared fictional stipulations, some acts are appropriate moves, others are inappropriate (i.e., mistakes)“(ebd.). Spiele sind regelbasierte Momente der sozialen und kognitiven Interaktion. Dieses Verständnis der menschlichen Fähigkeit, Fiktionen zu verstehen, findet auch auf literatur- und rezeptionstheoretischer Seite Beachtung. Hier gibt es bereits solche theoretischen Ansätze zur Erklärung des Fiktionsbegriffes, die - mit den Worten von Norbert Groeben und Ursula Christman - eine ,Interaktion` zwischen Leser- und Textmerkmalen bzw. eine mentale Interaktion zwischen Leser und Autor thematisieren (Christmann \& Groeben, 2014, S. 344). Eine Gruppe von Arbeiten, die diesem Kriterium entsprechen, sind institutionelle Fiktionalitätstheorien. Sie verdanken ihre Bezeichnung der Annahme, „dass die Eigenschaft bestimmter Texte (und anderer Medien), fiktional zu sein, auf einer sozialen Praxis koordinierten, konventionsbasierten Handelns beruht“ (Köppe, 2014, S. 35). Diese soziale Praxis des koordinierten und konventionsbasierten Handelns schließt den Textproduzenten ebenso ein wie den Textrezipienten. Ein fiktionaler Text wird demnach nicht nur mit der Absicht geschaffen, gemäß den Konventionen der Fiktionsinstitution rezipiert zu werden; es ist darüber hinaus entscheidend, dass der Leser während des Lektüreaktes dem Text gegenüber eine adäquate Rezeptionshaltung einnimmt (ebd.). Ausschlaggebend für eine solche angemessene Rezeptionshaltung gegenüber fiktionalen Texten ist das Befolgen bestimmter Regeln. Der Regelkatalog zur Fiktionsrezeption muss dabei sowohl dem Textproduzenten als auch dem Textrezipienten bekannt sein. Er enthält bestimmte Anforderungen an einen Leser, wie z. B. diejenige, „den Text als Grundlage einer imaginativen Auseinandersetzung mit dem Dargestellten zu nehmen und andererseits von bestimmten Schlüssen vom Text auf Sachverhalte in der Wirklichkeit [abzusehen]“ (ebd.). Damit ist gemeint, dass der Leser eines fiktionalen Werkes nicht davon ausgehen soll, dass die innerhalb des Werkes vorgefundenen Aussagen 
wahr sind. Leser sind allerdings dazu aufgefordert, die im Text dargestellten Inhalte zu,imaginieren' - eine kognitive Leistung, die im weiteren Verlauf dieser Arbeit genauer zu betrachten sein wird (ebd.).

Institutionelle Fiktionalitätstheorien basieren auf der grundlegenden Annahme, dass im Rahmen der Fiktionsrezeption eine ,mentale“ Interaktion zwischen Autoren und Lesern stattfinden muss, die die gegenseitige Attribuierung von konventionsbasiertem Wissen und einer grundlegenden intentionalen Haltung einschließt. Zu einer ähnlichen Schlussfolgerung gelangen die Vertreter sprechakttheoretischer Ansätze, wenn es darum geht, die spezifischen Eigenschaften fiktionaler Rede zu erfassen. Gregory Currie (1990, S. 11) schreibt in diesem Zusammenhang:

Fiction-making involves overt behavior. That is, it involves the production of some publicly accessible object that, $[\ldots]$ we may call a text. Fiction making is distinguished by the performance of a fictive utterance, an utterance produced in order to fulfill certain specific intentions; we may call them fictive intentions. $^{22}$

Da sich einzelne Sätze innerhalb von literarischen und nicht-literarischen Texten auf einer ,oberflächlichen‘ sprachlichen Ebene potenziell nicht voneinander unterscheiden lassen, liegt der entscheidende Unterschied demnach im ,Sprechmodus', bzw. der Sprechintention des Urhebers der betreffenden Aussage. Es ist die Haltung des Sprechers, die ihm zugeschrieben wird, die hier relevant ist (Searle, 2007). Das Bezugssystem eines Textes der einen fiktionalen Text schreibt, ist ein anderes als z. B. dasjenige eines journalistischen Textes. Es sind vordergründig nicht seine zwingend seine lexikalischen oder syntaktischen Eigenschaften, die einen Sprechakt im Kontext eines fiktionalen Textes von einem nicht-fiktionalen Sprechakt unterscheiden (ebd., S. 27). Der zentrale Unterschied zwischen fiktionalen Äußerungen und nicht-fiktionalen Äußerungen besteht laut Searle darin, dass die Autoren fiktionaler Textes lediglich vorgeben, einen gewöhnlichen illokutionären Akt zu vollziehen (ebd.). Damit ist nicht gemeint, dass Autoren fiktionaler Texte im Sinne einer Täuschungsabsicht vorgeben, eine bestimmte Aussage zu treffen. Das Verb ,vorgeben“ deutet vielmehr eine bestimmte Intention an, die von dem Leser eines fiktionalen Textes als solche erkannt werden muss. Gleichzeitig müssen Leser erkennen, dass diese intentionale Haltung von ihnen selbst eine entsprechende Rezeptionshaltung einfordert. Für Searle liegt das Kriterium

\footnotetext{
${ }^{22}$ Kursivierung durch den Autor. Die Begriffe, fictive utterance ${ }^{6}$ und ,fictive intentions ${ }^{6}$ meint hier also nicht ,erfundene‘ Äußerungen oder Intentionen, sondern Äußerungen, denen die Intention, Fiktionen zu produzieren zugrunde liegen bzw. die Intention, Fiktionen zu produzieren selbst.
} 
zur Identifikation eines fiktionalen Textes dementsprechend in der illokutionären Intention des Autors (ebd., S. 28). Eine fiktionale Geschichte ist also mit Searles Worten „[...] eine vorgebliche Darstellung einer Sachlage“ (Searle, 1982, S. 90 f.).

Beide hier kurz umrissenen fiktionstheoretischen Ansätze (der institutionelle und der sprechakttheoretische) enthalten den wichtigen Hinweis, dass die Intention des Textproduzenten und die Anpassung des eigenen Rezeptionsverhaltens seitens des Rezipienten für die Produktion und Rezeption fiktionaler Texte eine wichtige Funktion übernehmen. Sowohl der Urheber fiktionaler Sprechakte als auch ein Rezipient müssen für eine gelingende literarische Kommunikation eine bestimmte (intentional gelenkte) Haltung einnehmen. Damit geht jeweils eine Kognitionsleistung einher, die die Attribuierung einer bestimmten mentalen Haltung betrifft. Leser verstehen fiktionale Texte als fiktional, da sie den Urhebern der entsprechenden Sprechakte eine konkrete Handlungsmotivation, nämlich die Intention, ein fiktionales Werk zu schaffen, zuschreiben. Autoren wiederum wollen (im Regelfall), dass die Leser ihrer Texte diese als fiktional anerkennen. Sie setzen dabei voraus, dass Leser anerkennen, dass die Aussagen im Text in einem anderen Verhältnis zur Realität, aber auch z. B. zu den Überzeugungen des Autors stehen, als es bspw. im Fall des journalistischen Textes der Fall ist.

Es stellt sich die Frage, wie ein Leser diese spezifische Intention und Überzeugungshaltung des Autors erkennen kann, wenn es doch der Fall ist, dass sich die Aussagen innerhalb fiktionaler und nicht-fiktionaler Kontexte auf den ersten Blick z.B. anhand ihrer sprachlichen Eigenschaften häufig nicht voneinander unterscheiden lassen. Als kommunikative Hinweise zwischen Autoren und Lesern können verschiedene Fiktionssignale - also Merkmale, die die Fiktionalität eines Textes implizit oder explizit anzeigen - dienen. Solche Merkmale finden sich sowohl auf der Ebene der Geschichte (z. B. phantastische Ereignisse), auf der Ebene der Erzählung (z. B. metafiktionale Kommentare und Darstellungsformen, wie der innere Monolog) und auf paratextueller Ebene (z. B. Titel, Gattungsbezeichnung, Vorwort, Nachwort etc.). ${ }^{23}$ Solche Hinweise sind hilfreich um auf einer grundlegenden Ebene entscheiden zu können, welche Rezeptionshaltung einem Text gegenüber einzunehmen ist. Allerdings ist damit auch die Möglichkeit geschaffen, Leser über den Fiktionsstatus einer Aussage oder eines ganzen Textes in die Irre zu führen. Ein fiktionaler Text kann z. B. von seinem Autor (A) mit der Absicht verfasst werden, seinen Leser (L) über den fiktionalen

\footnotetext{
${ }^{23}$ Eine ausführliche Besprechung der Merkmale von Fiktionssignalen und unterschiedlicher Typologien wurde von Frank Zipfel (2001, S. 323 f.) erarbeitet.
} 
Status des Textes zu täuschen. In diesem Fall wäre die Attribuierung einer komplexeren Reihe mentaler Haltungen notwendig, um den Fiktionsstatus des Textes zu bestimmen (L erkennt, dass A tatsächlich intendiert, einen fiktionalen Text zu schreiben, aber den Fiktionsstatus des Textes verschleiert. L erkennt, dass A vorgibt, einen nicht-fiktionalen Text zu verfassen und L täuschen will). ${ }^{24}$ Umgekehrt betrachtet ist es ebenfalls möglich, dass ein Autor Aussagen in einen Text einbaut, die (bspw. gesellschaftskritische oder metapoetische) Referenzen enthalten und daher nicht so verstanden werden sollen, als bezögen sie sich lediglich auf die fiktionsinterne Realität. Wie entscheiden Leser über den Realitätsbezug einzelner Aussagen, wenn sich diese im Rahmen eines insgesamt als fiktional einzustufenden Textes befinden? In diesem Fall reicht eine einmalige Fiktionsbewertung mit Bezug auf den gesamten Text nicht aus. Die von den institutionellen und sprechakttheoretischen Fiktionstheorien postulierten ,Fiktionsintentionen“ und die unausgesprochenen ,Regeln“ der Fiktionsrezeption zwischen Autoren und Lesern helfen dabei, die kognitiven Grundhaltungen der an der literarischen Kommunikation beteiligten Instanzen besser zu verstehen. Woran erkennt ein Leser also, welcher Äußerung gegenüber er welche Rezeptionshaltung einnehmen muss? Wie bewertet ein Leser z. B. Fragen, die innerhalb fiktionaler Kontexte formuliert werden? Fragen erfüllen hier zum Teil eine andere kommunikative Funktion als im Rahmen einer realen Interaktion und dürfen im Regelfall nicht so verstanden werden, als wolle der Autor eines Textes etwas wissen (Köppe, 2014, S. 37). In zahlreichen Fällen ist eine differenziertere Auslegung solcher Fragen, die innerhalb von fiktionalen Kontexten stehen, möglich und notwendig. Um solche Fragen angemessen verstehen zu können, müssen Leser im Einzelfall differenziertere Aussageintentionen attribuieren, die sich auf isolierte Aussagen oder Passagen beziehen. Ein Beispiel hierfür ist die folgende Passage aus Herman Melvilles Moby Dick (1851), die sowohl eine (rhetorische) Frage, als auch eine Aussage enthält, die jeweils eine differenzierte Einschätzung durch den Leser gestatten, obwohl sie in einem Kontext stehen, der mit der Intention geschaffen wurde, ein fiktionales Werk zu schaffen. Die Passage lautet: „One often hears of writers that rise and swell with their subject, though it may seem but an ordinary one. How then with me, writing of this Leviathan?" (S. 432). ${ }^{25}$

\footnotetext{
${ }^{24}$ Autoren (auch Künstlern im Allgemeinen) steht die Möglichkeit offen, mit den Mitteln, die ihnen zur Verfügung stehen, ihre Rezipienten bewusst in die Irre zu führen. Ein bekanntes Beispiel, welches die Verwirrung von Rezipienten illustriert, die den Fiktionsstatus von Informationen nicht einzuschätzen wissen, sind die Reaktionen der Hörer einer amerikanischen Radiosendung, in der H.G. Wells War of the Worlds (1898) 1938 durch den Regisseur Orson Welles inszeniert wurde. (Vgl. z. B. den folgenden Zeitungsartikel, der die Reaktionen der Zuhörer auf die Radiosendung beschreibt: Mass Hysteria in U.S.A. Radio Broadcast Panic. The Age 2. November 1938. Abgerufen von https://news.google.com/newspapers?id=bJ1VAAAAIBAJ\& sjid=JJcDAAAAIBAJ\&pg=2609\%2C186374; zuletzt eingesehen am 12.05.2017.

${ }^{25}$ Die hier zitierte Ausgabe ist die folgende: Melville, H. (1994). Moby-Dick. London: Penguin Books.
} 
Herman Melville legt diese Worte dem Ich-Erzähler des Werkes, Ishmael, in den Mund. Die Passage ist jedoch so formuliert, dass sie ebenfalls für den eigentlichen Autor des Werkes selbst Gültigkeit beansprucht. Hier wird also eine Problematik geschildert, mit der sich nicht nur die fiktive Erzählerfigur, sondern auch der (durchaus reale) Autor konfrontiert sehen könnte. Es ist an dieser Stelle daher sinnvoll, die Informationen, die innerhalb dieser kurzen Passage vergeben werden, kognitiv sowohl mit einem Bezug zur Fiktionswelt, als auch mit einem Bezug zur realen Welt auszuwerten. Die hier involvierten Kognitionsleistungen können durch diejenigen kognitiven Ressourcen gestützt werden, die auch für die Zuweisung einer komplexen Reihe von mentalen Haltungen im realen sozialen Kontext zuständig sind. Leser sind in ihrer sozialen Interaktion offenkundig in der Lage, einer einzelnen Aussage wie in diesem Fall - zwei verschiedene Bezugsrahmen zu respektieren und damit auch zwei voneinander zu trennende Aussageintentionen zuzuweisen. Dies gelingt mit Hilfe einer der Kernfunktionen der ToM - der Attribution von Intentionen, die eine Person dazu veranlassen, eine bestimmte Aussage zu treffen. Ohne diese Fähigkeit wäre es kaum möglich, den besonderen Bezug zur Wirklichkeit einer Aussage innerhalb eines fiktionalen Textes einzuschätzen, da sie sich gegebenenfalls auf der Wortebene nicht von einer realitätsgebundenen Aussage unterscheiden lässt. Mit Blick auf die institutionellen und sprechakttheoretischen Fiktionsrezeptionstheorien ist also deutlich geworden, dass die angemessene Fiktionsrezeption und die ToM möglicherweise in einem Voraussetzungsverhältnis miteinander stehen: Ohne die ToM, d. h. ohne die Fähigkeit, dem Urheber einer Aussage oder eines Textes eine bestimmte Intention zuschreiben zu können, ist es nicht möglich zu entscheiden, welche Rezeptionshaltung einem Text oder einer bestimmten Aussage gegenüber eingenommen werden soll. Damit wäre die Rezeption literarischer Erzähltexte, die in dieser Arbeit im Zentrum des Interesses stehen, zumindest so wie wir sie kennen, nicht möglich. Umgekehrt ist es einem Autor ohne seine eigene ToM nicht möglich, die Reaktionen eines Lesers zu antizipieren und seiner Aussage/seinem Text die relevanten Kommunikationssignale (oder Fiktionssignale), einzuschreiben‘. Die ToM bildet hier die Voraussetzung für die ,mentale Verständigung ${ }^{`}$ zwischen Autor und Leser zu, denn sie schafft die Voraussetzung dafür, dass ein geeigneter Rezeptionsmodus für die Lektüre fiktionale Werke initiiert werden kann. Den Fiktionsstatus von Aussagen in fiktionalen Texten anzuerkennen ist jedoch nur eine von vielen Aufgaben, denen sich ein Leser stellen muss, wenn er einen literarischen Erzähltext rezipiert. Weiteren Aufgaben aus diesem Katalog möchte ich mich im Folgenden zuwenden. 


\section{Narrativität: Die Verarbeitung von narrativ vermittelten Informationen}

\subsection{Mentale Modelle als Basis von Verstehens- und Interpretationsleistungen}

Diejenigen Texte, die in dieser Arbeit im Fokus des Interesses stehen, sind literarische Erzähltexte. Neben anderen zentralen Eigenschaften, z. B. literarisch zu sein, zeichnen sie sich dadurch aus, dass in ihnen etwas erzählt wird - d. h. eine Abfolge von Ereignissen zusammen mit handelnden Figuren mit narrativen Mitteln dargestellt wird. Die Frage, die sich an dieser Stelle stellt, lautet, in welcher Form und mittels welcher kognitiver Mechanismen Leser narrative Inhalte verarbeiten und interpretieren. Hierfür ist das Zusammenspiel einer Reihe komplexer ToM-relevanter kognitiver Mechanismen relevant, denen ich mich nacheinander zuwenden möchte.

Ich habe bereits im ersten Teil der Arbeit beschrieben, dass sich bestimmte kognitive Inferenzen, die für die Lösung von ToM-relevanten Aufgaben notwendig sind, mit Hilfe der Theorie der mentalen Modelle erklären lässt. Diese kognitiven Grundlagen helfen ebenfalls dabei, zu verstehen, wie Leser das Geschehen innerhalb eines literarischen Erzähltextes kognitiv rekonstruieren. Um noch einmal kurz zu rekapitulieren: Ein zentraler kognitionswissenschaftlicher Ansatz besagt, dass Menschen ihre Umwelt mit Hilfe mentaler Modelle erfassen. Mit dieser Beschreibung der menschlichen Kognition lässt sich einleuchtend darstellen, wie z. B. das räumliche Gedächtnis des Menschen (Taylor \& Tversky, 1992) und Lernen durch Beobachten funktioniert (Magliano, Dijkstra \& Zwaan, 1996). Auch auf die Frage, inwiefern prädiktive und retrospektive Inferenzen im Zuge verschiedener ToM-relevanter Aufgaben wie z. B. der false-blief-Aufgabe möglich sind, lassen sich mit ihrer Hilfe Antworten finden. Es hilft ebenfalls dabei, zu verstehen, in welcher Form Leser das Geschehen innerhalb eines narrativen Textes kognitiv rekonstruieren. Wenn die Prinzipien der kognitiven Repräsentation in Form von mentalen Modelle in beiden Fällen die Verstehensprozesse lenken, dann wäre der Schluss gerechtfertigt, dass der ToM wie auch der Rezeption literarischer Erzähltexte zum Teil dieselben Kognitionsmechanismen zugrunde liegen. Wie ist das zu begründen?

Das der mentalen Modelle bereits in der Vergangenheit Eingang in die literatur- und kognitionswissenschaftliche Rezeptionsforschung gefunden. Allerdings steht in den relevanten 
Arbeiten die kognitive Verarbeitung von sprachlichen (schriftlichen) Stimuli im Allgemeinen und nicht notwendigerweise von literarischen (fiktionalen) Texten im Fokus (z. B. Kintsch 1998; Zwaan \& Radvansky, 1998). Da aber auch literarische fiktionale Texte in die übergeordnete Kategorie solcher Stimuli gehören, ist hier dennoch ein geeigneter Ausgangspunkt meiner Untersuchung gegeben.

Die Vertreter der Modell-Theorie nehmen an, dass sowohl sensorisch als auch sprachlich (mündlich oder schriftlich) vermittelte Informationen in Form von Modellen kognitiv repräsentiert werden (Johnson-Laird, 1983, S. 205 ff.). Auch Leser wandeln demzufolge die Informationen eines Textes mit Hilfe von eigenständigen Inferenzen in eine kohärente Textbasis (,text base) um: Kintsch und van Dijk beschreiben die Eigenschaften dieser kognitiven Textbasis genauer: „Propositions are ordered in the text base according to the way in which they are expressed in the text itself. Specifically, their order is determined by the order of the words in the text that correspond to the propositional predicates“" (Kintsch \& van Dijk, 1978, S. 367). Die Kohärenz der Textbasis wird durch einen Leser konstant überprüft. Ein Problem, das sich im Zuge dessen stellt, ist die limitierte Kapazität zur Repräsentation neu gewonnener Informationen. Das menschliche Arbeitsgedächtnis reicht schlicht nicht aus, um die Textbasis z. B. eines sehr umfangreichen Textes zu repräsentieren. Im Fall eines langen und entsprechend informationsreichen Textes ist es daher unwahrscheinlich, dass die gesamte Menge an Informationen gleichzeitig in diesen Prozess der ,Kohärenzprüfung‘ eingebunden ist. Kintsch und van Dijk schlagen daher vor, dass die textuell vermittelten Informationen in Sequenzen unterteilt und in einer Reihe von kognitiven Verarbeitungszyklen prozessiert werden. Die Anzahl der Propositionen, die einem solchen Zyklus zugeteilt werden, sind durch die strukturellen und semantischen ,Sinnabschnitte ${ }^{6}$ des individuellen Textes festgelegt (ebd., S. 368). Im Verlauf dieser Vorgänge wird die gesamte Menge der aufgenommenen Informationen bereits selektiert, um das Wesentliche des Textinhaltes herauszufiltern. Mit den Worten von Kintsch und van Dijk: „,[C]oherent text bases are constructed by a process operating in cycles and constrained by limitations or working memory. Macro processes are described that reduce the information in a text base through deletion and various types of inference to its gist“ (ebd., S. 389). Im Zuge dieser Prozesse entstehen drei verschiedene kognitive Repräsentationsebenen (Kintsch \& van Dijk, 1978, S. 237 f.). Dabei handelt es sich um (a) den Oberflächen-Code (also Wortlaut und Syntax, exakt wie sie im Text festgeschrieben sind), (b) die bereits beschriebene ,Textbasis' (also die Propositionen des Textes mit ihrem semantischen Gehalt, wobei für die Generierung von Kohärenz und Kohäsion möglicherweise eigene kognitiven Inferenzen seitens des Lesers zu leisten sind, 
um eventuelle Lücken des Kohärenzgefüges zu schließen) und (c) die kognitive Repräsentation des im Text Dargestellten. Letztere manifestiert sich in Form von kognitiven Situationsmodellen (, situation models ${ }^{6}$ ). Basierend auf dem semantischen Gehalt, der von sprachlichen Stimuli (wie z. B. Texten) vermittelt wird, bilden Leser kognitiv mentale Modelle, die - in Anlehnung an die einzelnen Sinnabschnitte eines Textes - ein oder mehrere räumlich und zeitlich begrenzte Ereignisse und die mit ihnen in Verbindung stehenden Objekte und Personen repräsentieren (Wyer, 2007, S. 288). In Abhängigkeit von der Strukturierung der textuellen Informationen bilden Leser während der Lektüre eines Textes eine alternierende Sequenz von Situationsmodellen. Im Arbeitsgedächtnis sind dabei nur diejenigen Modelle, die für das Verstehen der gegenwärtig aufgenommenen Informationen relevant sind ,zwischengespeichert‘. Welche einzelnen Modelle und Informationsgruppen für das Verständnis und die kognitive Rekonstruktion einer einzelnen Passage relevant sind, wird durch die Semantik der jeweiligen Textstelle festgelegt. Leser müssen sie aus der Menge bereits im Gedächtnis gespeicherter Modelle auswählen und abrufen. Die Reihenfolge, in der die Modelle verarbeitet und im Gedächtnis abgelegt werden, ist von der Abfolge der Informationsvergabe im Text (also allgemein gesprochen von einem Strukturmerkmal des Textes) abhängig.

Ein wichtiger Punkt, der die Informationsverarbeitung im sozialen Kontext von der Verarbeitung textuell vermittelter Informationen unterscheidet, ist, dass die Propositionen innerhalb eines Textes in einer festgelegten Reihenfolge vermittelt werden. Diese entspricht nicht notwendigerweise der natürlichen Reihenfolge der geschilderten Ereignisse, wie sie innerhalb der Abfolge der Situationsmodelle zu repräsentieren sind. In Erzähltexten ist es außerdem nicht möglich, gleichzeitig ablaufende Ereignisse auch gleichzeitig zu vermitteln. Die Vergabe der entsprechenden Informationen verläuft vielmehr linear nacheinander und die Repräsentation der geschilderten Ereignisse muss durch eine eigenständige Kognitionsleistung des Rezipienten dem semantischen Gehalt der vermittelten Informationen entsprechend restrukturiert werden, bevor ein kohärentes Situationsmodell gebildet werden kann. Besonders deutlich wird der Unterschied zwischen der Linearität der nacheinander dargestellten Ereignisse in einem Text und der Einbettung dieser Ereignisse in die dargestellte Zeitlichkeit innerhalb des Situationsmodells, wenn die natürliche Ordnung der geschilderten Ereignisse - wie in vielen literarischen Texten der Fall - umgestellt ist. Das bedeutet, dass die Informationsstruktur eines Erzähltextes und damit die Informationsaufnahme während der Lektüre sich nicht zwingend mit der Chronologie der dargestellten Ereignisse decken. Die Informationsaufnahme durch den Leser ist allerdings in einer bestimmten (und durch eine Textanalyse bestimmbaren) Art und Weise sequenziert. Ein Leser muss also neben der - in 
Genettes Terminologie - Ordnung in der Diegese eine Vorstellung von der zeitlichen Abfolge der Ereignisse gewinnen (Genette, ${ }^{3} 2010$, S. 18). Nach Gérard Genette lässt sich das Verhältnis der Darstellungschronologie und dem zeitlichen Verlauf innerhalb der erzählten Welt mit dem Begriff der, Ordnung` erfassen. Die Ordnung innerhalb einer Textstruktur beschreibt das Verhältnis zwischen der Anordnung der Ereignisse im narrativen Diskurs und der Abfolge derselben Ereignisse in der Fiktionswelt (ebd.). Sie legt fest, welche Informationen über welches Ereignis zu welchem Zeitpunkt während der Lektüre an den Leser vermittelt werden und beeinflusst damit maßgeblich die kognitive Modellierung des Geschehens seitens des Lesers. Die Gestaltung der Erzählchronologie legt fest, welche Informationen über einzelne Situationen und Figuren zu welchem Zeitpunkt während der Lektüre vermittelt werden. Es besteht also die Möglichkeit, dass ein Leser etwas über Ereignis B erfährt, wenn er über Ereignis A bereits informiert ist. Es besteht allerdings auch die Möglichkeit, dass er erst zu einem späteren Zeitpunkt der Lektüre etwas über Ereignis A erfährt als über Ereignis B usw. Leser sehen sich in letzterem Fall mit der Aufgabe konfrontiert, die natürliche Abfolge der Ereignisse im Zuge der Konstruktion eines Situationsmodells wiederherzustellen. Im Hinblick auf diese kognitive Leistungsanforderung wurde von anderen bereits die Hypothese formuliert, dass die Verarbeitungsgeschwindigkeit gegenwärtig aufgenommener Informationen davon abhängt, ob diese in das Situationsmodell, das zu diesem Zeitpunkt durch den Leser kognitiv repräsentiert wird, passen oder nicht. Wenn dies nicht der Fall ist, muss das passende Situationsmodell aus dem Gedächtnis abgerufen werden (Zwaan, 1999). In Abhängigkeit von der Konfiguration des Textes können dabei graduell abgestufte Diskontinuitäten in einer oder mehreren seiner Dimensionen auftreten. In diesem Sinne können die kognitiven Leistungsanforderungen von literarischen Texten also variieren.

Ein bekanntes und häufig genutztes Mittel, um solche kognitiven Leistungen erforderlich zu machen, ist der Einstieg in eine Geschichte medias in res. Texte dieser Art beginnen mit einem Ereignis, das in der Mitte oder am Ende des zeitlichen Ablaufes der Gesamthandlung liegt. Informationen, die zeitlich davorliegende Geschehnisse betreffen, werden dann im Verlauf der Erzählung in Form einer oder mehrerer Analepsen nachgereicht $(B-A-C)$. Ähnlich verhält es sich im Fall einer Prolepse. Hier steht innerhalb des natürlichen Verlaufs der temporalen Entwicklung ein Ausblick beziehungsweise ein Vorausgriff auf zukünftige Zustände der Fiktionswelt $(\mathrm{A}-\mathrm{C}-\mathrm{B})$. Ein Beispiel für eine Textpassage, die solche Kognitionsleistungen notwendig macht ist die Folgende. Diese Passage eröffnet Vladimir Nabokovs Werk Laughter in the dark (1933): 
Once upon a time there lived in Berlin, Germany, a man called Albinus. He was rich, respectable, happy; one day he abandoned his wife for the sake of a youthful mistress; he loved; was not loved; and his life ended in disaster.

This is the whole of the story and we might have left it at that had there not been profit and pleasure in telling [...].

It so happened that one night Albinus had a beautiful idea. (S. 7) ${ }^{26}$

Die Gesamthandlung wird hier im ersten Satz des Werkes vollständig vorweggenommen und bildet damit einen starken Interpretationsrahmen für die Verarbeitung aller nachfolgend vergebenen Informationen. Im Gegensatz zu dem Helden der Geschichte, weiß der Leser schon zu Beginn der Lektüre, dass sich das Schicksal der fiktiven Figur zum Schlechten wenden wird. Da sich Albinus über den weiteren Verlauf seines Lebens selbst nicht bewusst ist, müssen von Beginn der Lektüre an mindestens zwei verschiedene ,Versionen ‘ des entsprechenden Figurenmodells erstellt werden - eines, das einem früheren und eines, das einem späteren Entwicklungsstand der Figur entspricht. Der Leser muss, um alle nachfolgenden Textpassagen interpretieren zu können, jeweils diejenige ,Version` des Figurenmodells auswählen, die gemäß der ,natürlichen` Ereignisabfolge innerhalb des Fiktionsweltmodells Gültigkeit beansprucht. Dies ist insbesondere für die Attribution eines ,mentalen“ Profils, also zum Beispiel des ,gegenwärtigen“ (für den Leser ersichtlichen) Wissensstandes, ,gegenwärtiger` (für den Leser ersichtliche) Überzeugungen, Wünsche, Emotionen etc. relevant. Wenn die kognitive Leistung des Lesers zu einer kohärenten Abfolge von Modellen und zu stimmigen Figurenprofilen führt, können zum entsprechenden Zeitpunkt der Lektüre zugunsten eines kohärenten Textverständnisses Lücken durch die Attribution mentaler Haltungen geschlossen werden. Wie aber gelingt es einem Leser, die natürliche Abfolge der Ereignisse im Zuge der kognitiven Textrezeption zu rekonstruieren und ein kohärentes Situationsmodell zu erstellen, an Hand dessen vorausblickende Inferenzen gebildet werden können?

Ich habe bereits an anderer Stelle begründet, dass das ,event-indexing'-Modell (nachfolgend ,EIM`) von Zwaan et al. dazu geeignet ist, den Prozess der Ereignisrekonstruktion während der Lektüre eines literarischen Erzähltextes zu veranschaulichen (Luther, 2016a). Die Grundlage für die kognitive Modellierung narrativ dargestellter Ereignisse und Situationen bilden die einzelnen in einem Text zusammengestellten Propositionen und die durch sie be-

\footnotetext{
${ }^{26}$ Zitiert nach der folgenden Ausgabe: Nabokov, V. V. (1960). Laughter in the Dark. New York (NY): New Directions Publishing.
} 
dingt gebildeten Situationsmodelle (Zwaan, Langston \& Graesser, 1995). Während der fortlaufenden Lektüre müssen diese Modelle konstant mit Hilfe von hinzugewonnenen Informationen aktualisiert werden. ${ }^{27}$ Das gelingt, indem einzelne Propositionen aufgrund ihrer lexikalischen Merkmale in semantischen Einheiten, sogenannte ,events ${ }^{6}$ unterteilt werden. In jeder dieser einzelnen Einheiten sind Informationen gebündelt, die verschiedene (lokale, personale, temporale, motivationale etc.) Dimensionen einer bestimmten Situation beschreiben. Das EIM veranschaulicht, wie Leser textuell vermittelte Informationen mit Hilfe einer kognitiven Leistung umordnen, in Form von Situationsmodellen repräsentieren und auf diesem Weg ein kohärentes Verständnis darüber erlangen, was im Text geschildert wird. Leser können laut Kintsch und van Dijk im Zuge der Lektüre Propositionen mit einer Reihe verschiedener kognitiver ,Indices' kennzeichnen und so die entsprechenden Informationen in die bestehende Kohärenz der bereits gebildeten Situationsmodelle integrieren (Zwaan \& Madden, 2004). Die Indices selbst sind an lexikalische Hinweise durch Lokaladverbien, Personalpronomen, Ortsbezeichnungen u. ä. zur Einordnung von neuen Informationen in die bereits bestehende Matrix gebunden. Wenn also nacheinander beschrieben wird, welche Gegenstände sich in einem Raum befinden, können Leser mit Hilfe einer räumlichen Indizierung das entsprechende Objekt und den durch den Text zugewiesenen ,Ort` innerhalb des Modells miteinander verknüpfen. Ähnlich verhält es sich mit der zeitlichen Struktur eines Modells. Auch hier wird an Hand von lexikalischen Hinweisen (z. B. durch Temporaladverbien, Zeitangaben in Kapitelüberschriften o. Ä.) eine temporal strukturierte Ereigniskette rekonstruiert, die nicht der Darstellungsreihenfolge im Text entsprechen muss. Temporale Indices, die mit Hilfe von entsprechenden lexikalischen Hinweisen vergeben werden, zeigen an, ob ein berichtetes Ereignis A vor oder nach Ereignis B stattfindet und wie sich Ereignis $\mathrm{C}$ in die temporale Ordnung der Situationsmodelle einfügen lässt.

Leser verfolgen außerdem die wechselnde An- und Abwesenheit von Figuren in den einzelnen Handlungs- bzw. Ereignisabschnitten. Weitere wichtige Elemente der entstehenden Modelle sind die Repräsentationen von fiktiven Figuren. Figuren werden initiiert, indem sie an einem bestimmten Ort im Text bzw. zu einem bestimmten Zeitpunkt der Lektüre durch die Nennung eines Personalpronomens oder mit Hilfe von entsprechenden Vokabeln eingeführt werden. So beginnt Herman Melville sein Werk über den weißen Wal z. B. mit folgenden Worten: „Call me Ishmael. Some years ago - never mind how long precisely - having little

\footnotetext{
${ }^{27}$ Ich halte mich bei der Übersicht über den Event-Indexing-Ansatz hauptsächlich an die Ausführungen von Zwaan und Radvansky (1998). In dieser Studie finden sich auch Angaben zu empirischen Daten, die die Annahmen dieses Modells stützen.
} 
or no money in my purse, and nothing particular to interest me on shore, I thought I would sail about a little and see the watery part of the world“" (S. 21). Diese einführende Passage enthält Hinweise auf eine Entität mit dem Namen ,Ishmael ${ }^{‘}$, die die Eigenschaft besitzt, eigenständig denken (,I thought') und kommunizieren (,Call me...') zu können. Sie kann außerdem zukünftige Handlungen planen und Ziele verfolgen (,I thought I would sail a little and see the watery part of the world`). Sie genügt somit den Kriterien einer intentional und zielgerichtet handelnden anthropomorphen Figur und Leser können ein entsprechendes Modell initiieren, um diese Passage zu verstehen. Ist ein Figurenmodell einmal initiiert, wird es im Verlauf der Lektüre - also immer dann wenn ein Leser neue relevante Informationen erhält - mit Hilfe von entsprechenden Indices aktualisiert.

Ereignisse und Akteure sowie ihre räumliche und zeitliche Situierung bilden die grundlegenden ,Eckpfeiler‘ derjenigen Situationsmodelle, die es einem Leser ermöglichen, die Fiktionswelt kognitiv zu repräsentieren. Diese Grundbausteine werden durch explizite oder inferenzbasierte Informationen über kausale und handlungsmotivierende Relationen miteinander verbunden. Das EIM sieht daher zwei weitere Indices vor, die die kausale Verknüpfung einzelner Ereignisse sowie die Zuordnung von Handlungsplänen und -zielen zu den dargestellten Personen betreffen (Zwaan \& Radvanski, 1998, S.167 ff.). Hier stellt sich die Frage, ob die beiden letztgenannten Indices während des online-Rezeptionsprozesses einen gleichwertigen Status besitzen, wie die drei ersten. Schließlich können (und in vielen Fällen müssen) Schlüsse über kausale und handlungsmotivierende Zusammenhänge eigenständig durch den Leser gezogen werden, ohne dass sich eine entsprechende Proposition im Text findet. Wie bei Palmer und Zunshine bereits eindrücklich und an Hand verschiedener Textbeispiele belegt, sind Leser in zahlreichen Momenten der Textinterpretation angehalten oder sogar gezwungen, Schlüsse über kausale und motivationale Zusammenhänge zu ziehen, um ein literarisch dargestelltes Geschehen verstehen zu können. Zunshine (z. B. 2006, S. 22) argumentierte, dass Leser solche wichtigen handlungsmotivierenden Zusammenhänge dank ihrer ToM erschließen können und Autoren ihre Texte im Gegenzug so gestalten, dass ihre Leser an bestimmten Stellen der Textlektüre dazu eingeladen oder sogar gezwungen sind, ihre ToM für ein gelingendes Textverständnis einzusetzen. Ein zeitliches, personales und räumliches kognitives Koordinatensystem, das mit Hilfe von Indices im Sinne des EIM erstellt werden kann, ist eine geeignete Grundlage dafür, um Schlüsse über kausale Zusammenhänge, Handlungsziele bzw. handlungsmotivierende intentionale Faktoren ziehen zu können, wie an anderer Stelle bereits ausführlicher beschrieben (Luther, 2016a). Eine mögliche 
Erklärung dafür, dass Leser und Autoren ihre ToM im Zuge der Textrezeption bzw. Textproduktion in vergleichbarer Art und Weise einsetzen können wie innerhalb realer sozialer Interaktionen ist, dass in beiden Fällen Informationen in Form kognitiver Modelle repräsentiert werden. Mit Hilfe dieser Modelle lassen sich große Informationsmengen in kohärenter Form bündeln und abrufen. Damit sind alle Voraussetzungen dafür erfüllt, dass Leser - ähnlich wie im realen sozialen Kontext - sowohl zeitlich rückblickende Schlüsse ziehen als auch vorausblickende Vermutungen über Handlungsentwicklungen aufstellen können. Diese Form der kognitiven Informationsverarbeitung bietet also einen geeigneten Rahmen für diejenigen Schlussfolgerungen über soziale Handlungen, mentale Haltungen und innere Vorgänge, die einen großen Teil der menschlichen Fähigkeit ,eine ToM zu haben` auszeichnen.

Mein vorläufiges Fazit nach den vorangehenden Überlegungen lautet also: Der reale soziale Verstehenskontext und die Lektüre eines literarischen Erzähltextes sind hinsichtlich der Informationsstruktur, die einem Menschen zur Verfügung stehen, unterschiedlich. Kognitive Situationsmodelle bilden einen möglichen ,common ground ${ }^{‘}$. Mit anderen Worten: Trotz der initialen Unterschiede in der Informations- und Vermittlungsstruktur der beiden Kontexte, können Informationen jeweils so aufbereitet werden, dass sowohl im realen sozialen Kontext als auch im Kontext der iterarischen Rezeption eine vergleichbare Grundlage für die Informationsverarbeitung besteht.

Die Attribution einer generellen Fiktionsintention und die darauf basierende flexible Einschätzung des Realitätsbezuges einzelner Aussagen innerhalb eines Textes bilden einen wichtigen Teil der Aufgaben eines Lesers, der einen literarischen Erzähltext zur Hand nimmt und zu lesen beginnt. Die Ereignisse, Objekte und Personen, die in einem Text geschildert werden, kognitiv zu rekonstruieren - sie sich vorzustellen - gehört ebenfalls zu diesem Aufgabenkatalog. Das EIM und die Theorie der Bildung mentaler Situationsmodelle ist hilfreich um einen Teil dieser Prozesse beschreiben zu können. Die imaginative Auseinandersetzung mit literarischen Inhalten ist damit jedoch noch nicht erschöpfend erklärt. Daher wende ich mich im Folgenden derjenigen Ebene der literarischen Rezeption zu, die die kognitive Rekonstruktion von Wahrnehmung betrifft. Jerome Bruner (1986) wies bereits darauf hin, dass es neben der ,Ereignis‘-Ebene eines literarischen Textes eine weitere wichtige Dimension gibt, die ein Leser bei der Interpretation des Textes berücksichtigen muss. Er bezeichnet die beiden unterschiedlichen Ebenen in Anlehnung an eine bereits von Greimas und Courtes vorgeschlagene Differenzierung als, landscape of action' und ,landscape of consciousness'. Er schreibt dazu: 
[A] story must construct two landscapes simultaneously. One is the landscape of action, where the constituents are the arguments of action: agent, intention or goal, situation, instrument, [...]. The other landscape is the landscape of consciousness: what those involved the the action know, think, or feel, or do not know, think or feel. (ebd. S. 14)

Im vergangenen Abschnitt dieser Arbeit lag der Fokus auf der kognitiven Situations- und Ereignisrekonstruktion, die entweder durch reale Stimuli oder die Lektüre eines literarischen Textes angeregt und gesteuert wird. Im Folgenden wende ich mich der Frage zu, inwiefern Leser anthropomorphe Instanzen im Rahmen der literarischen Rezeption kognitiv mit einer eigenständigen Wahrnehmung und einer eigenen Perspektive ausstatten können.

\subsection{Die kognitive Konstruktion von Wahrnehmung / die perspektivgebundene Rekonstruktion fiktiver Ereignisse}

Bevor die kognitive Rekonstruktion von Perspektivität und die Rolle der ToM im Zuge dessen näher beleuchtet werden kann, stellt sich allerdings die Frage, was genau mit diesem Begriff bezeichnet wird. Ansgar Nünning (2001) zufolge ist der Begriff der Figuren- bzw. Erzählperspektive nicht nur an eine räumlich-zeitliche Koordinate innerhalb des Fiktionsweltmodells gebunden (Wer sagt wann was), sondern ebenfalls an eine Reihe von unterschiedlichen Daten über die Konfiguration der informationsvermittelnden Instanz:

A character-perspective [and by analogy to the term character-perspective, the concept of narrator-perspective] could [...] be defined as an individual's fictional system of preconditions or subjective worldview - the sum of all the models he or she has constructed of the world, of others, and of herself. A character-perspective is governed by the totality of an individual's knowledge and belief sets, intentions, psychological traits, attitudes, ideological stance, and system of values and norms that have been internalized [...]. (Nünning, 2001, S. 211)

Das Verstehen von literarisch vermittelten Informationen ist (vergleichbar wie subjektiv gebrochen vermittelte Informationen in der realen sozialen Interaktion) an bestimmte perspektivische Parameter gebunden. Leser müssen diese Parameter bei ihrer Interpretation berücksichtigen. Konkreter gesagt: Sie müssen den semantischen Gehalt aller in einem bestimmten Kontext vergebenen Informationen daraufhin prüfen, in welchem Verhältnis sie zum Urhe- 
ber der Aussage stehen und in welche Beziehung sie zum bestehenden Modell der Fiktionswelt zu setzen sind. Aus der Perspektive von Figur A bedeutet ein Satz X ggf. nicht dasselbe, wie derselbe Satz, wenn er von Figur B oder dem Erzähler ausgesprochen wird. Bei der Interpretation dieses Satzes spielen daher zum Beispiel der Wissenstand, der Wahrnehmungszugang und die intentionale Grundhaltung, um nur einige Beispiele zu nennen, die dem Urheber einer Aussage innerhalb des Textes zugeschrieben werden, eine entscheidende Rolle. Hat ein Leser solche Parameter identifiziert, bedingen sie das Verständnis einer literarischen Passage in entscheidender Form. Die beginnende ontogenetische Entwicklung bzw. Reifung der für das Erkennen der Perspektivgebundenheit von Wahrnehmung und Handlung relevanten kognitiven Mechanismen zeigt sich erstmals im kindlichen Verhalten des ,joint attention` (,JA`). Wie im ersten Teil der Arbeit näher beschrieben, sind Menschen ca. ab ihrem 12. Lebensmonat in der Lage, Objekte in einen gemeinsamen und koordinierten Aufmerksamkeitsfokus mit einer oder mehreren Bezugspersonen zu stellen. Dieses soziale Verhalten wird hauptsächlich durch eine Reihe kommunikativer deiktischer Gesten (z. B. Zeigegesten), der Körperhaltung der Beteiligten oder der Fähigkeit, Blickrichtungen zu folgen, ermöglicht und gesteuert. Mittels dieser Verhaltensweisen entsteht eine triadische Wahrnehmungssituation, die sich folgendermaßen charakterisieren lässt: „Each subject is aware, in some sense, of the object as an object that is present to both subjects. There is, in this respect, a ,meeting of the minds' between both subjects, such that the fact that both are attending to the same object is open or mutually manifest" (Eilan, 2005, S. 5). Während jede an einer solchen Interaktionssituation beteiligte Person ein Objekt aus der jeweils eigenen Perspektive repräsentiert, steht gleichzeitig auch der ,mentale Fokus‘ des jeweiligen Gegenübers im Zentrum der eigenen Aufmerksamkeit. Um einen Gegenstand als Objekt fremder Wahrnehmung repräsentieren zu können, müssen bestimmte perspektivgebundene Parameter berechnet werden. Mit anderen Worten: Von einem Standpunkt der Person A stellt sich ein Gegenstand gegebenenfalls anders dar als von einem Standpunkt der Person B. Um dies kognitiv verarbeiten zu können, müssen wir nicht nur den Gegenstand selbst unter Berücksichtigung einer fremden Perspektive rekonstruieren, sondern ihn ebenfalls sowohl als Objekt der eigenen als auch als Objekt fremder Wahrnehmung anerkennen. Gesunde Erwachsene sind dazu in die Lage, den Wahrnehmungskontext eines anderen zu repräsentieren und die Erscheinung ein und desselben Gegenstandes unter Berücksichtigung verschiedener Perspektiven kognitiv zu modellieren. Im Zuge einer JA-Episode beziehen sie die individuelle Perspektive des Gegenübers in das Situationsverständnis ein, um die fremde Wahrnehmung 
zu verstehen. Dieser Mechanismus bildet einen wichtigen Teil der kognitiven ,Architektur der ToM.

Wenn es die Informationsstruktur eines literarischen Textes gestattet, wird dieser Mechanismus auch dann aktiviert, wenn die Aufgabe lautet, literarisch vermittelte Perspektivität zu verstehen und in die Interpretation eines Textes einzubinden. Ob das der Fall ist, lässt sich mit einem Blick auf eine Reihe von bestimmten Merkmalen literarischer Erzähltexte klären. Auch hier - wie im Fall der kognitiven Ereignisrekonstruktion - fallen Unterschiede zwischen der Kognitionssituation der Textlektüre und derjenigen der realen sozialen Interaktion auf und es gilt zu klären, ob aufgrund dessen grundlegend andere Kognitionsmechanismen für die Textinterpretation notwendig sind. Wenn sich im Zuge einer JA-Situation die Wahrnehmung zweier Individuen auf ein Objekt bzw. auf die Wahrnehmung dieses Objektes durch den jeweils anderen richtet, dann wird dies zumeist mit Hilfe von interaktiven kommunikativen Strategien (v. a. nonverbale Mittel wie Zeigegesten, Mimik, Blickkontakt usw.) initiiert und gesteuert. Im Zuge der Textlektüre kann jedoch keine direkte Interaktion zwischen Lesern und dem Kommunikationspartner (z. B. dem Autor des Textes oder auch einer fiktiven Instanz innerhalb des Textes) im Sinne des JA stattfinden. Es besteht kein zeitgleicher und gleichwertiger Zugang zu einem gemeinsamen Wahrnehmungskontext. Der literarische Text selbst lässt sich jedoch, wie an anderer Stelle bereits näher ausgeführt, als Vermittlungsmedium beschreiben, durch das z. B. Autoren und Leser in eine ,mentale Interaktion“ miteinander treten können. Die grundlegende Annahme, die dieser Überlegung voransteht lautet, dass Autoren und Leser im Zuge der Textproduktion und -rezeption mentale Modelle von Situationen repräsentieren, die durch die textuellen Propositionen initiiert und aktualisiert werden. Die in ihnen zu einem komplexen Fiktionsweltmodell zusammengestellten Informationen bilden einen gemeinsamen Referenzrahmen für einen Autor und seinen Leser. Mit anderen Worten: Als Ersatz für die reale kommunikative Interaktion finden sich in den Propositionen eines Textes sprachlich realisierte ,Zeigegesten“. Der Zugang zu diesem Wahrnehmungskontext ist nicht perzeptueller Natur, sondern entsteht durch die gegenseitige Attribution von kognitiven Interpretations- und Repräsentationsvorgängen. Der Text als Vermittlungsmedium dient in diesem Kontext als ,Anleitung‘ zur geordneten Rekonstruktion des dargestellten Geschehens unter Berücksichtigung der jeweils relevanten Perspektiven.

Eine geeignete Erklärung für die Vorgänge, die es einem Leser ermöglichen, Perspektiven im Rahmen der Lektüre zu rekonstruieren, bietet die Deictic Shift Theory (,DST') an. Die 
zentrale These der DST lautet, dass Leser während der Lektüre eines literarischen Erzähltextes das eigene deiktische System der Realität in ihrer Wahrnehmung gegen dasjenige der Fiktionswelt eintauschen:

DST models the common perception of a reader ,getting inside' a literary text as the reader taking a cognitive stance within the mentally constructed world of the text. [...] In other words, readers can see things virtually from the perspective of the character or narrator inside the text-world, and construct a rich context by resolving deictic expressions from that viewpoint. (Stockwell, 2005, S. 46 f.)

Zusätzlich zu der Verlagerung des deiktischen Orientierungssystems in die Fiktionswelt hinein (also die Übernahme desjenigen Koordinatensystems, das innerhalb der Fiktionswelt gültig ist), ist es dem Leser nun möglich, innerhalb der Fiktionswelt verschiedene deiktische Bezugspunkte zu modellieren, wenn die Informationsstruktur des Textes dies vorgibt. Hierfür, d. h. für die Konstruktion unterschiedlicher, Wahrnehmungsfixpunkte', sind eine Reihe von lexikalischen Hinweisen notwendig, die dem Leser das Gefühl vermitteln, dass Figuren, die Erzählinstanz und der Leser selbst das fiktive Geschehen von einem jeweils festgelegten ,Standpunkt' aus betrachten. Es sind vor allem lexikalische Hinweise, z. B. durch die Verwendung von Personalpronomen, Demonstrativpronomen und direkte Referenzen aber auch Verben, wie denken oder glauben, die mentale Zustände beschreiben, die die Grundlage für die Identifikation und Lokalisation von Figuren und Gegenständen im Gefüge der Fiktionswelt steuern. Entsprechend situieren lokale sowie temporale Präpositionen und Adverbien die Figuren im zeitlichen und räumlichen Gefüge der Handlung.

Die wichtigsten Mittel, die hier die kognitive Rekonstruktion von unterschiedlichen perspektivischen Standpunkten innerhalb einer Fiktionswelt bedingen, sind die deiktischen Strukturen eines Textes. Sie ersetzen die kommunikativen ,Zeigegesten“ innerhalb einer direkten sozialen Interaktion. Als deiktisch werden Zeichen oder Ausdrücke dann bezeichnet, wenn sie keine festgelegte semantische Bedeutung tragen, sondern eine, die sich relativ zu dem Kontext verhält, in der dieses Zeichen oder dieser Ausdruck steht. Die Interpretation eines solchen Zeichens oder Ausdruckes ist in der Identität des Sprechers, des Angesprochenen, deren räumlicher Situation sowie dem Zeitpunkt der Äußerung verankert (Zubin \& Hewitt, 1995, S.129). Ein Ausdruck wie zum Beispiel ,Du musst an der nächsten Ecke links abbiegen' lässt sich dementsprechend nur dann korrekt interpretieren, wenn die deiktischen Elemente der Passage ,Du' ,links' und , an der nächsten Ecke' sowohl zu einem räumlich-zeitlichen Koordinatensystem als auch zu den Identitäten von Sprecher und Adressat in Relation 
gesetzt werden können. Kognitive Modelle von fiktiven Szenarien besitzen ebenso wie Modelle von realen Szenarien ein räumlich-zeitliches bzw. deiktisches Gefüge, zu dem alle Informationen über stattfindende Ereignisse und handelnde Personen in Relation gestellt werden können. Jeder Leser, der zunächst einmal mit dem eigenen Hier und Jetzt seiner Realität ein deiktisches Koordinatensystem zur Orientierung besitzt, muss daher ein eigenes solches Koordinatensystem für die Fiktionswelt erstellen. In dieses Koordinatensystem kann er (z. B. mit Hilfe eines räumlichen, zeitlichen, kausalen, personalen oder motivationalen Index) die Figuren, die die jeweilige Fiktionswelt bewohnen, und die Gegenstände, die zu dieser Welt gehören, einordnen. Zubin und Hewitt schreiben: „Just as constructing a mental model of the story world involves importing world knowledge into the interpretation of fictional text, so the deictic construction of narrative is derivative of the reader's experience of deictic centering in the here/now/I/you of the everyday world“" (ebd., S. 131). Ein Leser kann daher - wenn er Aussagen innerhalb des Textes auf das deiktische Koordinatensystem der Fiktionswelt bezieht - auf vergleichbare Art und Weise prädiktive Schlüsse ziehen, wie innerhalb der realen Welt. Hierfür muss er jedoch die geschilderten Ereignisse konstant auf ein deiktisches Zentrum beziehen, das nicht mit seinem eigenen übereinstimmt. Das ,Links` in einer bestimmten Situation innerhalb der Fiktionswelt stimmt allerdings nicht notwendigerweise mit dem gegenwärtigen ,links‘ der eigenen Realität überein. Das muss ein Leser bei der Interpretation einer Aussage im Text berücksichtigen. Er muss sich sozusagen in die fiktive Welt ,hineinversetzen“. Der Wechsel des deiktischen Bezugssystems, also dieses ,Hineinversetzen` schafft eine Ähnlichkeit der Voraussetzungen für die kognitive Informationsverarbeitung im Rahmen realer Kontexte und im Zuge der Lektüre eines literarischen Textes, die mittels der kognitiven Modellierung von Situationen erzielt wird. Die vergleichbare Verarbeitung von textuell vermittelten Informationen wiederum ist eine mögliche Begründung für die subjektive Empfindung von Lesern, die in der wissenschaftlichen Literatur bereits unter den Stichworten ,transportation', ,loss of self-awareness ${ }^{6}$, ,flow $^{\star}$ und ,identification von mehreren Seiten besprochen wurde. ${ }^{28}$

Warum ein Leser im Zuge der Textlektüre dazu angehalten ist, ,die Perspektive zu wechseln', lässt sich am besten an Hand eines Beispiels illustrieren. Die nachfolgend zitierte Passage aus Virginia Woolfs Mrs. Dalloway (1925) veranschaulicht, inwiefern das subtile deiktische Webmuster eines Textes den Leser zwingt, eine Situation aus verschiedenen Blickrichtungen oder Wahrnehmungsstandpunkten zu rekonstruieren. Sein eigener Fokus wird

\footnotetext{
${ }^{28}$ Für eine umfassendere Zusammenfassung der verschiedenen Ansätze zur Erklärung dieses Effekts vgl. Buselle \& Bilandzic (2008), S $260 \mathrm{f}$.
} 
dabei durch die impliziten ,Zeigegesten“ der deiktischen Feinstruktur der Textpassage gesteuert:
„Who can - what can,” asked Mrs. Dalloway (thinking it was outrageous to be interrupted at eleven o'clock on the morning of the day she was giving a party), hearing a step on the stairs. She heard a hand upon the door. She made to hide her dress, like a virgin protecting chastity, respecting privacy. Now the brass knob slipped. Now, and in came - for a single second she could not remember what he was called! So surprised she was to see him, so glad, so shy, so utterly taken aback to have Peter Walsh come to her unexpectedly in the morning! (She had not read his letter.)
„And how are you?” said Peter Walsh, positively trembling; taking both her hands; kissing both her hands. She's grown older, he thought, sitting down. I shan't tell her anything about it, he thought, for she's grown older. She's looking at me, he thought, a sudden embarrassment coming over him, though he had kissed her hands. Putting his hand into his pocket, he took out a large pocket-knife and half opened the blade.
Exactly the same, thought Clariszsa; the same queer look; the same check suit; a little out of the straight his face is, a little thinner, dryer, perhaps, but he looks awfully well, and just the same. (S. 45) ${ }^{29}$

Bei der Lektüre dieser kurzen Passage müssen Leser zunächst drei distinkte wahrnehmende Instanzen identifizieren, wobei zwei davon sowohl durch Namen als auch Personalpronomen eindeutig gekennzeichnet sind. Bei der dritten Instanz handelt es sich um die Erzählinstanz selbst, die sich durch den Akt des Erzählens selbst zu erkennen gibt und dem Leser vermittelt, dass Peter seine Hand in seine Tasche steckt, ein Taschenmesser herausnimmt und die Klinge zur Hälfte öffnet. Den Perspektivwechsel zwischen den beiden interagierenden Figuren initiiert jeweils das Mentalverb ,thought', ergänzt durch einen Namen oder ein Personalpronomen. Eine exakte Zeitangabe ,eleven o'clock on the morning of the day she was giving a party', situiert das Geschehen im temporalen Nexus der Gesamthandlung des Werkes. Das zweifach gereihte Temporaladverb ,now‘, sowie die parallele Syntax der Sätze: ,She heard a hand upon the door. She made to hide her dress, like a virgin protecting chastity, respecting privacy. Now the brass knob slipped. Now the door opened [...]' gliedern die innere zeitliche Abfolge dieser kurzen Textpassage. Um die Art der Beziehung der beiden Figuren einordnen zu können, stehen direkte Informationen über die Gefühlsreaktion des weiblichen Charakters zur Verfügung. Sie ist überrascht, erfreut, verlegen und erstaunt, zu diesem Zeitpunkt an diesem Ort Peter zu sehen. Die Umbruchstelle zwischen den beiden

\footnotetext{
${ }^{29}$ Die zitierte Ausgabe ist die Folgende: Woolf, V. (1960). Mrs. Dalloway. London: Hogart Press.
} 
Innensichten der beteiligten Figuren ist scharf gezeichnet. Sieht der Leser die Situation zunächst einmal ,durch die Augen' Clarissas, zwingt ihn die Deixis des Textes zum Verlagern des Fokus auf Peters Gedankenwelt. Diese Verlagerung, der so genannte Shift, wird allein durch das Mentalverb ,thought', in Verbindung mit einem Namen (in diesem Fall ,Peter ${ }^{`}$ ), ausgelöst. Dabei wird eine Verlagerung der Wahrnehmungsperspektive und damit des ,Standpunktes“ innerhalb des deiktischen Koordinatensystems des Textes initiiert. In diesem speziellen Fall wird durch die direkte Darstellung von Gedankengängen eine Verbindung zwischen dem kognitiv generierten Blickwinkel des Lesers auf die geschilderten Ereignisse und dem textuell vermittelten Blickwinkel der Figur statt, so dass Leser - so kommt es ihm vor - die fiktive Situation aus der Perspektive der jeweiligen Figur erfassen können. Diese Strategie ist nur eins von zahlreichen denkbaren Beispielen. Im Zuge einer kognitivistischen Textanalyse ist es notwendig nach den jeweils individuellen Strukturen zu fragen, die die kognitive Rekonstruktion von Situationen, Ereignissen und Wahrnehmungsperspektiven lenken.

In den vergangenen Kapiteln ist deutlich geworden, dass Leser trotz der oberflächlichen Unterschiede in den Informationsstrukturen und -Medien die ToM im Zuge der Textlektüre ebenso für die Interpretation des geschilderten Geschehens einsetzen können, wie im realen sozialen Kontext. Wenn Menschen zu Gunsten solcher Verstehensprozesse mentale Modelle bilden, dann entstehen vergleichbare Kognitionsbedingungen für entsprechende prädiktive oder attributive Interpretationsakte. Außerdem bilden die besonderen Mechanismen der ToM wichtige Voraussetzungen der literarischen Rezeption so wie wir sie kennen. Sie erlauben es uns, Handlungsmotivationen zu entschlüsseln, Perspektivität an Hand von deiktischen Hinweisen innerhalb eines Textes zu berechnen und generelle Aussageintentionen zu attribuieren. Ein möglicher Grund hierfür könnte sein, dass literarische Werke in der Regel auch von Menschen geschaffen werden, die über eine funktionierende ToM verfügen.

\subsection{Die grundlegende Rekursivität narrativ vermittelter Informationen}

Literarische Erzähltexte zeichnen sich neben den unterschiedlichen perspektivischen Standpunkten innerhalb der geschilderten Situationen u. a. auch dadurch aus, dass diese Situationen erzählerisch vermittelt werden. Es gibt also eine Instanz - entweder eine konkrete fiktive Figur, eine anthropomorphe Erzählinstanz oder im Fall einer Nullfokalisierung schlicht eine Erzählstimme, durch die einem Leser die gegebenen Informationen vermittelt werden. Leser 
nehmen die Situationen, die in literarischen Erzähltexten geschildert werden, also nicht direkt und wie im realen Kontext unvermittelt mit Hilfe ihrer Sinnesorgane wahr. Es ist für den Akt des Erzählens selbst also konstitutiv, dass ,[j]edes Ereignis, von dem in einer Erzählung erzählt wird, [...] auf der nächsthöheren Ebene zu der [liegt], auf der der hervorbringende narrative Akt dieser Erzählung angesiedelt ist [sic]“" (Genette, 2010, S. 148). ${ }^{30}$ Neben der grundlegenden und für die literarische Rezeption konstitutiven Informationsvermittlung zeichnen sich literarische Erzähltexte also auch durch ihr Potenzial aus, verschiedene ineinander gestaffelte Erzähl-,Ebenen` zu eröffnen. Dies geschieht indem z. B. innerhalb der erzählten Situation eine Figur ebenfalls eine Geschichte erzählt. Damit können literarische Erzähltexte unterschiedliche, voneinander zu trennende, ineinander geschachtelte Erzählebenen aufweisen und es ist in solchen Fällen Aufgabe des Lesers diese Ebenen und ihren Bezug zueinander korrekt zu entschlüsseln. Parallel zu einer solchen verschachtelten Erzählstruktur gewinnt der kognitive Rezeptionsakt an Komplexität. Die Interpretation der innerhalb der Geschichte geschilderten Handlungen hängt z. B. entscheidend von der Bewertung des Geschichtenerzählers ab, der zum Beispiel als unzuverlässig eingeschätzt werden kann. Ganz konkret bedeutet das, dass die Täuschungsabsicht eines extradiegetischen Erzählers (E1) zum Beispiel Konsequenzen für die Evaluation aller durch sie vergebenen Informationen hat, also auch Verarbeitung derjenigen Informationen betrifft, die sich auf untergeordnete fiktionale Ebenen beziehen. Die Täuschungsabsicht eines intradiegetischen Erzählers (E2) gilt seinem direkten Ansprechpartner innerhalb der Fiktionswelt und betrifft die Evaluation entsprechend übergeordneter Informationen, die durch E1 vergeben werden, nicht.

Leser müssen also in der Lage sein, einen (fiktiven) Sachverhalt X zu interpretieren, wenn die Erzählinstanz erster Stufe (E1) angibt, dass die Erzählinstanz zweiter Stufe (E2) behauptet, Y sei der Fall und der Leser über die Unzuverlässigkeit von E1, E2 oder beiden (sei es auf Grund von Wissenslücken oder Täuschungsabsichten) informiert ist. Ein besonders anschauliches literarisches Beispiel, das es seinem Leser abverlangt, ein Fiktionsweltmodell mit mehreren in bestimmter Art und Weise zueinander in Beziehung stehenden Ebenen zu konstruieren, ist House of Leaves (2000) von Mark Z. Danielewski. Das Werk beginnt mit dem Bericht des jungen Tattookünstlers Jonny Truant (E1) darüber, dass er die Abhandlung des blinden und kürzlich verstorbenen Zampanò (E2) über den Dokumentarfilm des Photojournalisten Will Navidson (Erzählebene 3) findet, redigiert und herausgeben möchte. Jede

\footnotetext{
${ }^{30}$ Hervorhebungen durch den Autor.
} 
einzelne Erzählebene enthält, in sich eingebettet, die Handlungen der nächst höheren Ebene. Bei einer solchen Verschachtelung mehrerer Erzählebenen entsteht eine komplexe Konstellation aus Sprechern und Adressaten. Gleichzeitig entsteht eine komplexe Struktur rekursiver Bezüge, da die erzählten Inhalte durch eine Erzählinstanz auf einer nächst höheren Erzählebene vermittelt werden. Aufgabe eines Lesers ist es in diesem Fall, diese Strukturen korrekt zu repräsentieren und die semantischen Merkmale der einzelnen eingebetteten Ebenen ihrem Status innerhalb des, geschachtelten“ Fiktionsweltmodells angemessen zu interpretieren.

Das oben genannte Beispiel zeichnet sich durch besondere Komplexität aus und illustriert die möglichen kognitiven Herausforderungen während der Lektüre eines literarischen Erzähltextes besonders anschaulich. Auch einfach vermittelte Texte zeichnen sich jedoch durch mindestens eine einfache Einbettungsstruktur aus. Die Struktur, die dadurch entsteht, dass nicht einfach etwas geschieht, sondern ein Erzähler erzählt, dass etwas geschieht, halte ich für ein inhärentes Merkmal literarischer Texte. Leser sind daher in jedem Fall mit der Aufgabe konfrontiert, mindestens eine Ebene, in vielen Fällen sogar mehrere Ebenen der Einbettung zu entschlüsseln. Im Fall realer Ereignisse gilt, dass etwas tatsächlich der Fall ist. Im Fall literarisch vermittelter Ereignisse hingegen gilt, dass eine Erzählinstanz oder eine Erzählstimme angibt, etwas sei der Fall.

Die auffälligen Korrelationen mit grammatisch-syntaktischen Fähigkeiten (z. B. dem Verstehen von Komplementsätzen) haben im Zuge der ToM Forschung gezeigt, dass das Verstehen rekursiver Strukturen für bestimmte ToM-Fähigkeiten eine wichtige Rolle spielt. Ausschlaggebend war hier ein genauer Blick auf die Struktur und die Funktion der einzelnen Abschnitte von Komplementsätzen, die sich ebenfalls durch die Einbettung inhaltlicher Informationen in einen funktionalen Satzteil auszeichnen. Erzählakte lassen sich - genau wie Situationen, die auf mentalen Haltungen basieren [z. B. A denkt, X sei der Fall] - in Komplement-Formeln [E sagt, X sei der Fall] ausdrücken. Der Wahrheitswert des Komplementsatzteils ist grundsätzlich unabhängig vom einleitenden Satzteil, steht allerdings in einem bestimmten semantischen Bezug zu ihm. Damit vergleichbar sind im Fall von literarischen Erzähltexten Informationen, die eine bestimmte Situation betreffen in einen Erzählakt eingebettet. Um in einem literarischen Erzähltext dargestellte Ereignisse korrekt rekonstruieren zu können, muss die Erzählsituation bzw. müssen die Eigenschaften der Erzählinstanz berücksichtigt werden. Im Fall von Komplementsätzen muss der funktionale Satzteil des Sat- 
zes zu dem Inhalt des eingebetteten Satzteils in Beziehung gestellt werden, um ihn zu verstehen. Das legt die Vermutung nahe, dass auch bei der Interpretation der in literarischen Erzähltexten vermittelten Ereignisse - wie auch im Zuge des Verstehens fremder mentaler Haltungen - ein kognitiver Mechanismus relevant ist, der es uns gestattet, rekursiv eingebettete Informationen verarbeiten zu können. Ein solcher Mechanismus dient in beiden Fällen dazu, im Zuge der Interpretation die unterschiedlichen Ebenen einer kognitiven Repräsentation getrennt voneinander zu bewerten und zueinander in eine Beziehung zu stellen. Die auf diese Weise gebildete Relation zwischen den Ebenen bedingt die Interpretation der vermittelten Informationen sowohl im Fall von literarisch-narrativ vermittelten Informationen als auch im Zuge des Verstehens von einfachen Komplementsätzen.

Damit ist eine zentrale strukturelle Gemeinsamkeit zwischen der Kognitionssituation im Zuge der Lektüre und einer wichtigen Dimension der ToM-Fähigkeiten identifiziert. Diese Gemeinsamkeit besteht darin, das Leser und Beobachter realer sozialer Interaktionen gleichermaßen in der Lage sein müssen, rekursiv eingebettete Informationen entschlüsseln zu können. Das ist nicht nur deshalb der Fall, weil in literarischen Texten häufig bereits auf der Inhalts- oder Handlungsebene soziale Handlungen (die auf mentalen Haltungen beruhen) geschildert werden.

Die Überlegungen der vergangenen zwei Unterkapitel bezogen sich auf typische Eigenschaften und Merkmale literarischer Texte, die mit ihrer Narrativität und dem Akt des Erzählens im Zusammenhang stehen. Im Folgenden möchte ich ein weiteres essenzielles Merkmal literarischer Erzähltexte in den Fokus der Aufmerksamkeit stellen - ihre Literarizität.

\section{Die metakognitive Verarbeitung literarischer Informationen}

In dieser Arbeit steht die kognitive Rezeption literarischer Erzähltexte im Fokus des Interesses. Die bereits geleistete kognitionswissenschaftliche Forschung bezieht sich, wie bereits erwähnt, überwiegend auf narrative Texte im Allgemeinen. Daher stellt sich nun die Frage, ob die Literarizität oder Poetizität eines Textes, den wir als ,literarisch`klassifizieren würden, besondere Relevanz für die kognitive literarische Rezeption hat. Um diese Frage beantworten zu können, ist es unerlässlich, zunächst einmal zu klären, ob - und wenn ja inwiefern - sich die Literarizität eines Textes manifestiert. Das ist deshalb schwierig, weil der literaturwissenschaftliche Diskurs über dieses Problem unabgeschlossen ist und sich überwiegend 
durch eine definitorische Uneinheitlichkeit der Begriffsbestimmung auszeichnet. So vergleicht Simone Winko die Frage nach dem alle literarischen Texte vereinenden Literarizitätskriterium passend mit der Suche nach der Weltformel. Gleichzeitig stellt sie nach einer umfassenden Sichtung des Forschungsstandes fest, dass die Frage, ob es immanente Eigenschaften und Funktionen von Texten gibt, die sie zu literarischen machen, zu verneinen ist. Sie schreibt: „Kein Kandidat für ein textinternes Literarizitätskriterium hat allgemeine Zustimmung erhalten, und selbst für minimalistische Lösungen ist ein disziplinärer Konsens ausgeblieben“ (Winko, 2009, S. 374). Trotzdem ist es im Sinne der Beantwortung der eben gestellten Frage zum Einfluss der Literarizität eines Textes auf den kognitiven literarischen Rezeptionsprozess notwendig, einige Überlegungen aus der Diskussion um den Literarizitäts-Begriff zusammenzutragen. Damit sollen Hinweise auf die Wirkung von Texteigenschaften und die kognitive literarische Rezeption offengelegt werden, ohne den eigentlichen Diskurs um den literaturtheoretischen Grundbegriff zu einem versöhnlichen Abschluss führen zu wollen oder einen neuen definitorischen Standpunkt zu entwickeln. Winko schließt den innerhalb ihres Beitrages geleisteten detaillierten Forschungsüberblick zum Literarizitätsbegriff mit dem Fazit, dass der Versuch, die textinternen Kriterien als notwendige und hinreichende Bedingungen für Literatur zu postulieren, als gescheitert angesehen werden kann. Es ist, so Winko, nicht der Fall, dass jeder Text, der eine Reihe von spezifischen Merkmalen aufweist, zur Literatur zählt und dass sich auf der anderen Seite in allen der Literatur zugesprochenen Texten diese Merkmale manifestieren. Es gibt im Gegenzug literarische Werke, deren formale Eigenschaften sie nicht von Texten unterscheiden, die gemeinhin als nicht-literarisch gelten (ebd., S. 391). Oder, um es mit Terry Eagletons Worten zu umschreiben: „Alles kann Literatur sein, und alles, was als unwandelbar und unbestreitbar als Literatur angesehen wird - Shakespeare, zum Beispiel - kann eines Tages keine Literatur mehr sein“ (Eagleton, 1996, S. 12).

Trotz dieser unbefriedigenden Ergebnisse bezüglich der Frage nach einem haltbaren begrifflichen Konzept der Literarizität wird die Suche nach Definitionsansätzen dennoch aktiv weiterverfolgt. Dabei gewinnen empirische Untersuchungsmethoden im Vergleich zur theoretischen Diskussion immer mehr an Relevanz. Im Rahmen empirischer Studien wird nicht nach einer Lösung für das Problem der Begriffsdefinition gesucht. Hier steht vielmehr die Frage im Zentrum des Interesses, inwiefern sich ,Literarizität' nicht in textinhärenten Merkmalen, sondern in einem bestimmten Rezeptionsmodus manifestiert (Miall, 2006, S. 312). Auf der anderen Seite steht die Frage nach denjenigen Textmerkmalen oder kontextuellen Umständen, die einen solchen Rezeptionsmodus seitens des Lesers initiieren können. 
Es gibt empirische Ergebnisse, die daraufhin deuten, dass die literarische Rezeption von Lesern als besondere Erfahrung gegenüber der Lektüre von nicht-literarischen Texten empfunden wird (Ebd., S. 292). Eine mögliche Begründung bzw. Schlussforderung aus dieser Beobachtung lautet, dass im ersten Fall eine Reihe besonderer Kognitionsprozesse gefordert sind oder stimuliert werden. Wie genau lässt sich das erklären? Hier ist es erneut hilfreich mit Hilfe der Theorie der mentalen Modelle auf die Struktur der kognitiven Prozesse zu blicken. Während der Erstellung von mentalen Modellen werden Wyer zufolge immer auch Informationen über die Modalität der zugrunde liegenden Informationen kodiert (Wyer, 2007, S. 291). Das hat zur Folge, dass die Sinneseindrücke, die mit einer beobachteten oder ,erfahrenen' Situation einhergehen, ebenfalls kognitiv kodiert werden und wieder abrufbar sind.

Trifft das zu, dann ließen sich folgende Überlegungen im Hinblick auf die kognitive Rezeption literarischer Texte anstellen: Hier wird - im Gegensatz zum realen sozialen Kontext ein Situationsmodell mit Hilfe von textuell vermittelten Informationen erstellt. Wenn im Zuge dessen Daten über die Art der Informationsvermittlung in die kognitive Informationsverarbeitung einfließen, wären sie in diesem Fall als metalinguistisch zu beschreiben. Literarische Texte bilden allerdings eine besondere Gruppe von Texten. Sie haben zwar u. a. den sprachlichen (schriftlichen) Vermittlungsmodus mit anderen Texten gemeinsam, unterscheiden sich von anderen Texten aber dadurch, dass ihnen das Merkmal der Literarizität zugesprochen (attribuiert) wird. Durch eine solche Attribution werden die Informtionen, die während der Rezeption eines als literarisch betrachteten Textes verarbeitet werden, entsprechend indiziert.

Auf der anderen Seite wird im Zuge der Forschungsgeschichte der Literaturwissenschaft immer wieder von der, Besonderheit‘ der literarischen Sprache gegenüber der alltäglichen Umgangssprache gesprochen. Miall und Kuiken untersuchten aus diesem Grund die Annahme, ob und inwiefern formale und strukturelle Merkmale literarischer Sprache, ihre Rezeption beeinflussen. Sie skizzieren die Zielsetzung ihrer Studien mit den folgenden Worten: „By studying the experience of literary reading and its outcomes, we will begin to map the interaction between reader and text and discover what formal structures created within that interaction warrant reference to such reading as ,literary““ (Miall \& Kuiken, 1998, S. 328). Ausgangspunkt für ihre Überlegungen und Anhaltspunkte für mögliche Alleinstellungsmerkmale literarischer Sprache sind die zentralen Thesen des russischen Formalismus. Die 
Vertreter dieser Schule stellten die literarische Sprache selbst - und damit ein objektiv beschreibbares und empirisch erfassbares Merkmal literarischer Texte - in den Mittelpunkt der Aufmerksamkeit. Innerhalb literarischer Texte, so das Fazit von Jakobson (1979, S. 119) in einem paradigmatischen Aufsatz dazu, weicht die Sprache systematisch von der in nichtliterarischen Texten vorzufindenden Alltagssprache ab und verdient deshalb z. B. unter eigenen wissenschaftlichen Gesichtspunkten untersucht zu werden. Eigenschaften wie Dichte, Rhythmus und Klang der in literarischen Kontexten verwendeten Worte und Wortkombinationen dominieren ihm zufolge die literarische Wahrnehmung gegenüber einer reinen Bedeutungsrekonstruktion des Gelesenen. Literarische Sprache - im Gegensatz zu nicht-literarischer Sprache - lenkt die Aufmerksamkeit ihres Lesers auf sich selbst als materielle Substanz eines Textes (Eagleton, 1996, S. 2). Mit Viktor Shklovskys Worten:

The purpose of art is to impart the sensation of things as they are perceived and not as they are known. The technique of art is to make objects ,unfamiliar", to make forms difficult, to increase the difficulty and length of perception because the process of perception is an aesthetic end in itself [...]. (Shklovsky, 1965, S. 12)

Basierend auf diesen Überlegungen ließe sich vermuten, dass der kognitive Leistungsaufwand während der Verarbeitung inhaltlicher Informationen bei einem gleichzeitigen Bewusstsein für die strukturellen Eigenschaften der Sprache ein anderer ist, als im Fall der durchlässigen, sich selbst ,unsichtbar' machenden nicht-literarischen Sprache, die in nichtliterarischen Kontexten zu verorten ist. Jan Mukařowský, der sich ebenfalls mit diesem Gedanken befasst, zieht im Anschluss daran eine erste relevante Schlussfolgerung für die Auswirkungen auf die kognitive Rezeption eines literarischen Textes. Auch er benennt mit der ,Entautomatisierung' bekannter Sprache-Bedeutungs-Relationen eine zentrale Funktion poetischer Sprache. Daran anschließend hält er fest: „Foregrounding is the opposite of automatization, that is, the deautomatization of an act; the more an act is automatized, the less it is consciously executed; the more it is foregrounded, the more completely conscious does it become" (Mukařowský, 2000, S. 226).

Miall und Kuiken stellen im Anschluss daran die berechtigte Frage, ob eine ,entautomatisierte' Sprachverwendung im Kontext literarischer Texte tatsächlich einen Effekt für die kognitiven Verarbeitungsprozesse eines Lesers hat. Ihre zentrale Vermutung lautet: „,[...] that the novelty of an unusual linguistic variation is defamiliarizing, defamiliarization evokes feelings, and feelings guide ,refamiliarizing“ interpretative efforts“ (Miall \& Kuiken, 1994, S. 391). Ihre Ergebnisse fußen auf den in mehreren einzelnen Versuchsreihen gesammelten 
Daten, in denen Testteilnehmern ausgewählte und mit Hilfe eines Experten-Bewertungsverfahrens als besonders ,literarisch` kategorisierte Textsegmente präsentiert wurden. Im Verlauf der jeweiligen Lektürephase wurde anschließend einerseits die Lesezeit bezüglich der einzelnen Textausschnitte gemessen und andererseits mit einer Wertungsskala das Niveau der Besonderheit oder Auffälligkeit (,strikingness ) einzelner Passagen gegenüber nicht-literarischen Texten erhoben.

Die gemessenen Ergebnisse aus den verschiedenen Studien, die durchgeführt wurden, um die aufgestellte Hypothese zu überprüfen, bestätigten die angestellten Vermutungen:

Our analysis of reader's responses to several literary texts (short stories and poems) indicates processes beyond the explanatory reach of current situation models. Such findings suggest a three-component model of literariness involving foregrounded stylistic or narrative features, reader's defamiliarizing responses to them, and the consequent modification of personal meanings. (Miall \& Kuiken, 1999, S. 121)

Mjall und Kuikens Ergebnisse zeigen, dass - vorausgesetzt ein Text enthält Sprache, die von der gewöhnlichen Alltagssprache abweicht - die Wahrnehmung vordergründiger formaler Aspekte der Sprache ein wichtiger Bestandteil der kognitiven literarischen Rezeption ist. Durch ungewohnte sprachliche Strukturen wird die die Re-Evaluation bekannter Bedeutungsmuster auslöst und geht ein ,Entfremdungs'-Effekt provoziert (Miall \& Kuiken, 1998; Miall \& Kuiken, 1999). Das ist ein Indikator dafür, dass während der Lektüre eines literarischen Textes, dessen Materialität durch künstlerische Mittel in den Vordergrund gestellt sind, seitens des Lesers nicht nur Repräsentationen der vermittelten fiktiven Ereignisse entstehen. Vielmehr werden, wenn der Bezug von textuellen Informationen zum fiktiven Ereignis zu Gunsten eines Selbstbezuges abnimmt, möglicherweise auch Informationen auf einer ,meta- ' Ebene verarbeitet.

Selbstredend sind ungewöhnliche ,ent-automatisierte' sprachliche Elemente nicht das einzige denkbare Merkmal, das einen literarischen von einem nicht-literarischen Text unterscheidet. Darüber hinaus lässt sich ebenfalls argumentieren, dass es in vielen Fällen schwierig ist, in Texten, die wir als literarisch ansehen, solche Sprachstrukturen zu identifizieren, die sich eindeutig von der Alltagssprache unterscheiden. Dennoch geben die oben beschriebenen Studien und Überlegungen Anlass dazu, eine Hypothese zu formulieren. Ähnlich wie gegenüber der Fiktionalität eines Textes, ist es denkbar, dass Leser, die einen Text als ,literarisch` anerkennen, eine besondere Rezeptionshaltung einnehmen. Diese kann z. B. durch 
außergewöhnliche Sprache, aber auch durch den Rezeptionskontext (z.B. Hinweise auf dem Umschlag des Buches), Paratexte oder einer Vorprägung des Lesers (z.B. durch Vorwissen) und andere Merkmale des Textes ausgelöst werden. Wenn die Verarbeitung sprachlicher Informationen mit Hilfe mentaler Modelle die Verarbeitung metalinguistischer Informationen einschließt, dann ist es in Erweiterung dieses Gedankens möglich, dass die Verarbeitung literarischer Informationen metaliterarische Informationen einschließt. Das würde bedeuten, dass die literarische Rezeption von zwei Kognitionsebenen getragen wird - einer, auf der die geschilderte Fiktionswelt modelliert wird und einer weiteren, auf der die Informationen über diejenigen Textmerkmale kodiert werden, die den literarischen Text als solchen kennzeichnen. Ließe sich dies im Rahmen eines empirischen Versuches nachweisen, sind zwei verschiedene Anschlussfragen interessant: Welche Textmerkmale führen dazu, dass eine solche zweite Rezeptionsebene entsteht? Sie würden aus rezeptionspsychologischer Sicht mögliche Unterscheidungsmerkmale zwischen literarischen und nicht-literarischen Texten darstellen. Und: Müssen Leser bewusst eine bestimmte Rezeptionshaltung einnehmen, bevor sie mit der Lektüre beginnen, oder können Leser, die unvoreingenommen an einen unbekannten Textausschnitt herantreten, allein durch die Beschaffenheit des Textes selbst dazu gebracht werden, eine solche zweite Kognitionsebene zu initiieren? Diese Fragen sind im Rahmen einer theoretischen Arbeit nicht zu beantworten. Die z. B. von Mjall und Kuiken festgehaltenen Beobachtungen bilden jedoch das Fundament dafür, eine Hypothese über eine denkbare Parallele zwischen ToM und der literarischen Rezeption zu formulieren. In beiden Fällen - so scheint es - spielt die Verarbeitung von Informationen auf einer MetaEbene eine Rolle. In diesem Zusammenhang müssen Menschen in der Lage sein, Informationen über die (reale oder fiktive) Welt von Informationen über den Vermittlungsmodus dieser Informationen zu ,entkoppeln‘ ${ }^{31}$ Ein solcher Mechanismus ist sowohl für zentrale Funktionen der ToM (z. B. wenn Menschen im Zuge des Fiktionsspiels einer Repräsentation unterschiedliche Bezüge zur Wirklichkeit zuordnen können) als auch im Zuge der Rezeption literarischer Texte relevant. Das ist eine weitere Begründung für die u.a. von Zunshine (2006) und Daniel D. Hutto (2009) geäußerten These gefunden, dass die Lektüre literarischer Erzähltexte die ToM eines Menschen ,trainieren‘ kann. Die Erklärung, dass Menschen, die viele literarische Erzähltexte lesen, schlicht Gelegenheiten haben, eine größere Anzahl von möglichen Szenarien zu interpretieren, ließe sich dann weiter spezifizieren. Die ToM von

\footnotetext{
${ }^{31}$ Dabei sei erst einmal dahingestellt, ob Menschen dies grundsätzlich bewusst oder unbewusst bzw. situationsabhängig bewusst oder unbewusst leisten.
} 
Viellesern wird auch deshalb, trainiert‘ weil neben der zusätzlichen Gelegenheit zur Interpretation psychologischer Handlungen auch durch literaturspezifische, auf den ersten Blick nicht-mentale Ereignisse, kognitive Mechanismen aktiviert werden, die für die ToM-relevant sind. Ich möchte diese Hypothese nicht aus dem Blick verlieren, wenn ich mich im Folgenden einer letzten Aufgabe der kognitiven literarischen Rezeption zuwende, die hier bereits mehrfach erwähnt, aber nicht eingehender betrachtet wurde. Gemeint ist die imaginative Auseinandersetzung mit literarisch dargestellten Fiktionswelten, auf die sich Leser literarischer Texte einlassen.

\section{Fiktionsrezeption und die kognitive Repräsentation imaginativer Gegenstände}

\subsection{Die Imaginationsleistung und der eigene Standpunkt gegenüber der Fiktionswelt}

Köppe bezeichnete den fiktionalen Text als „Grundlage für eine imaginative Auseinandersetzung mit dem Dargestellten“ (Köppe, 2015, S. 35). Das bedeutet: Wenn ein Leser den Text als fiktional eingestuft hat, erkennt er u. a. auch die Aufgabe an, sich das dargestellte Geschehen vorzustellen. Bereits mit Blick auf die Entwicklung des kindlichen Fiktionsspiels (Kapitel 2.3 dieser Arbeit) wurde ein solcher Mechanismus beschrieben. Er liegt laut Rakoczy (2008, S. 1195) in der Fähigkeit begründet, institutionelle Regeln zu bilden und sich situationsabhängig ihnen zufolge zu verhalten. Dieser Gedanke, der von Rakoczy im Kontext der Entwicklungspsychologie aufgegriffen wird, baut u. A. auf älteren Überlegungen von Kendall Walton auf, die mit besonderem Fokus auf die Produktion und Rezeption sämtlicher darstellender Kunstwerke formuliert wurden (u. A. Walton, 1990, S. 11). Waltons grundlegende Annahme lautet, dass sich die Fiktionswahrnehmung im Rahmen des kindlichen pretend play und die Fiktionswahrnehmung von darstellenden Kunstwerken in entscheidenden Punkten ähnlich sind (Walton, 2007, S. 100). Beide Tätigkeiten, sowohl die Beschäftigung mit darstellenden Kunstwerken als auch die Teilnahme am Fiktionsspiel, generieren ihm zufolge Regeln, die im jeweiligen Kontext gültig sind. Gleichzeitig werden bestimmte Propositionen stipuliert: Im Kontext von Fiktionswelt A gilt z. B., dass X wahr 
ist und $\mathrm{Y}$ nicht wahr ist. Im Kontext von Fiktionswelt B gilt hingegen, dass $\mathrm{Y}$ und $\mathrm{Z}$ wahr sind.

Generiert werden solche Propositionen und Regeln im Fall des Fiktionsspiels u. a. durch die Verwendung von Requisiten, die Walton Props nennt (z. B. ein Ast, den ein Kind im Rahmen einer Spielepisode zu einem Schwert , uminterpretiert ${ }^{`}$ ). Dabei stehen die Eigenschaften eines realen Gegenstandes in direktem Zusammenhang mit seiner imaginierten Funktion innerhalb der Fiktionswelt. Die Propositionen, die einen fiktionalen Text konstituieren, sind laut Walton ähnlich zu verstehen wie die innerhalb des Fiktionsspiels verwendeten Requisiten: Eine zentrale Gemeinsamkeit zwischen spielerischen Requisiten und fiktionskonstituierenden Propositionen ist z. B. ihre respektive Eigenschaft, eine Person dazu anleiten zu können, sich etwas vorzustellen. Mit Waltons eigenen Worten:

Representations, I have said, are things possessing the social function of serving as props in games of make-believe, although they also prompt imaginings and are sometimes objects of them as well. A prop is something which, by virtue of the conditional principles of generation, mandates imaginings. Propositions whose imaginings are mandated are fictional, and the fact that a given proposition is fiction is a fictional truth. Fictional worlds are associated with collections of fictional truths; what is fictional is fictional in a given world the world of a game of make-believe, for example, or that of a representational work of art. (Walton, 1990, S. 69) 32 $^{32}$

Ähnlich wie Kinder im Zuge des Fiktionsspiels folgen Leser fiktionaler Texte dem Auftrag, sich etwas vorzustellen. Dieser Auftrag ist nicht vollständig frei, sondern als regelgeleitet zu verstehen, da die lexikalischen und strukturellen Eigenschaften der Propositionen eines Textes (wie die Requisiten im Fiktionsspiel) Leser dazu ,anleiten' sich etwas Bestimmtes in bestimmter Form vorzustellen. ${ }^{33}$ Lesern stehen also, wie hier von Walton beschrieben, die Menge aller vom Autor verwendeten Propositionen zur Beschreibung einer bestimmten Fiktionswelt zur Verfügung, um das Grundgerüst einer imaginativen Welt kognitiv zu errichten (die Fiktionswelt kognitiv zu modellieren). Zu einer auf diese Weise generierten kognitiven Repräsentation einer Fiktionswelt nehmen Spielteilnehmer wie Leser laut Walton eine bestimmte kognitive Haltung ein, wobei sie ,so tun als ob' die entsprechenden imaginativen

\footnotetext{
${ }^{32}$ Alle Hervorhebungen im Original.

${ }^{33}$ Inwiefern empirische Leser diese Anleitung umsetzen kann an dieser Stelle mit den zur Verfügung stehenden Mitteln nicht untersucht werden. Diese Frage bleibt deshalb zukünftigen empirischen Arbeiten vorbehalten.
} 
Inhalte im Kontext der Fiktionswelt wahr seien. Es ist für einen Leser im Rahmen der Fiktionswelt von Gullivers Travels (1726) beispielsweise wahr, dass es eine Gesellschaft aus sehr kleinen Menschen gibt, die deshalb Krieg miteinander führen, weil sie sich uneinig sind, an welchem Ende ein Ei richtigerweise aufgeschlagen werden muss (ebd. S. 102). Die Imaginationsleistung, die dem Fiktionsspiel und der Beschäftigung mit Kunstwerken zu Grunde liegt, geht also damit einher, dass wir den Bezug zu Relatität, der überlicherweise unserer kognitive Informationsverarbeitung lenkt, teilweise oder ganz ,suspendieren“. Kinder können während einer Spielepisode beispielsweise die reale Eigenschaft eines Astes, zwei Spitzen zu haben, zu Gunsten der fiktiven Eigenschaft des imaginären Schwertes, nur eine Spitze zu haben, suspendieren. Parallel dazu suspendieren die Leser von Gullivers Travels ihr Wissen, dass es in Wirklichkeit keine Gesellschaft aus sehr kleinen Menschen gibt, die einen Krieg führen, weil sie sich uneins darüber sind, an welchem Ende ein Ei richtigerweise aufgeschlagen werden muss. Während die Behauptung, es gäbe eine solche Gesellschaft im Raum der imaginierten Fiktionswelt also wahr ist, ist sie im Raum der wirklichen Welt nicht wahr. Ein Leser muss beide Welten kognitiv trennen. Das gelingt, indem er (zeitlich begrenzt) lediglich ,so tut als ob“ die fiktive Proposition wahr sei. Da er nur so tut, als sei sie wahr, beeinflusst sie die eigene Überzeugung oder das Wissen über die Realität nicht oder nur mittelbar. Um ein einfaches Beispiel zu nennen: Wenn ein Kind im Rahmen eines Fiktionsspiels einen imaginären Regenschirm aufspannt, führt dies nicht automatisch zu der Überzeugung seines Spielpartners es regne tatsächlich. ${ }^{34}$ Ein Kind, das an einer Episode des Fiktionsspiels teilnimmt, stellt sich vor, Teil der Fiktionswelt zu sein. Es stellt sich vor, dass es selbst den Regenschirm aufspannt oder selbst, gegen den Bären kämpft‘. Es stellt sich vor, Handlungen auszuführen, die es in der Wirklichkeit nicht ausführt bzw. lediglich mit Hilfe von Requisiten simuliert. Das bedeutet, dass im Rahmen des Fiktionsspiels oder während der Lektüre eines fiktionalen Textes eigene Perspektiven und Emotionen im Bezug auf das literarisch dargestellte oder im Ragmen des Fiktionsspiels stipulierte Geschehen kognitiv generiert werden können. Ein Kind, das sich in seiner Vorstellung also einem Bären entgegenstellt, generiert fiktionale Wahrheiten über sich selbst, womit das Kind selbst zu einem imaginativen Teil der eigenständig erschaffenen Fiktionswelt wird (ebd., S. 213). Ähnliches gilt für die Beschäftigung mit darstellenden Kunstwerken. Auch ein darstellendes Kunstwerk lädt dazu ein, die dargestellten Ereignisse, Figuren und Gegenstände, die nicht Teil der Realität sind, ,zu betrachten‘. Besonders aufschlussreich ist hier das von Walton gewählte

\footnotetext{
${ }^{34}$ Vorausgesetzt es handelt sich um einen gesunden und entsprechend weit entwickelten Menschen, der versteht, dass die gesehene Handlung im Kontext einer Spielepisode steht.
} 
Beispiel des Betrachters eines Gemäldes, das eine Landschaft zeigt (ebd., S. 215 f.). Walton argumentiert: „The museum goer who looks at Willem Van der Velde's landscape Shore at Sheveningen $[\ldots]$ in the normal manner makes it fictional of himself that he is looking at a group of sailing ships approaching a beach on which there is a horse-drawn cart“ (ebd., S. 215) ${ }^{35}$ Waltons Museumsbesucher nimmt also nicht nur wahr, dass er in einem Museum steht und ein Bild betrachtet, das eine bestimmte Landschaft zeigt, er kann sich ebenfalls vorstellen, die dargestellte Szenerie selbst zu betrachten. Das bedeutet im Umkehrschluss nicht, dass der Betrachter des Bildes damit zu einem tatsächlichen Teil des Bildes werden muss. Er kann sich allerdings vorstellen, die Szenerie so wahrzunehmen, als sei er selbst Teil der Fiktionswelt. Ähnliches gilt für den Leser eines fiktionalen Werkes, der nicht tatsächlich miterlebt, wie Ahab Jagd auf den weißen Wal macht, der sich aber vorstellen kann, Ahab dabei zu beobachten, wie er Jagd auf den weißen Wal macht. Wenn dies der Fall ist, dann hätte diese Form der ,aktiven Imagination“ wichtige Konsequenzen für die involvierten kognitiven Prozesse, die an der Fiktionsrezeption beteiligt sind.

Wenn wir uns mittels unserer Imagination in eine bestimmte fiktive Situation hineinversetzen, dann ist es möglich, dass damit eine emotionale Beteiligung einhergeht, die mit der emotionalen Beteiligung an realen Situationen vergleichbar ist. Die Frage, die sich Walton stellt, lautet, ob die einer fiktiven Situation empfundenen Emotionen identisch mit denjenigen sind, die wir gegenüber einer realen Situation empfinden. Schließlich ist der Bezugsrahmen, der die Emotion auslöst, jeweils ein anderer. Ein weiterer Unterschied besteht darin, dass, um einer Fiktion gegenüber eine Emotion zu generieren, eine Imaginationsleistung vorausgesetzt ist. Ein Leser muss einer Figur nicht nur bestimmte mentale Haltungen zuschreiben, er muss sich diese Figur erst vorstellen, um ihr dann eine bestimmte mentale Haltung zuschreiben zu können. Walton schließt daraus, dass ein Leser nicht tatsächlich an einer fiktionalen Situation emotional teilhat, sondern lediglich in seiner Vorstellung Angst, Mitgefühl, Schrecken o. Ä. erlebt. Allerdings stehen ihm für die Simulation dieser Vorgänge im Rahmen der Fiktionswelt teilweise dieselben kognitiven Ressourcen zur Verfügung wie im Rahmen der realen Welt. Damit sind die mentalen Vorgänge, die ein Leser in die Imaginationswelt hineinträgt, zwar nicht identisch mit denjenigen, die sich auf die reale Welt beziehen, sie basieren aber zum Teil auf den gleichen kognitiven Vorgängen. Mit Waltons Worten:

\footnotetext{
${ }^{35}$ Hervorhebungen durch den Autor.
} 
We do not actually [...] grieve for Anna Karenina [...] nor do we feel contempt for Iago or worry for Tom Sawyer and Becky lost in the cave. But it is fictional that we do. It is fictional, when we appreciate novels, plays, films, and paintings that we feel compassion, exasperation, indignation, and so on [...]. And these fictional truths are generated in a similar manner - in part by our actual mind. (Ebd., S. 249 f.)

Damit sind Leser bei der Lektüre eines fiktionalen Textes im Rahmen ihrer eigenen Imagination insofern aktiv, als sie ihre kognitiven Ressourcen dazu einsetzen, innerhalb der Fiktionswelt angemessene eigene emotionale Reaktionen oder Überzeugungshaltungen zu generieren. Walton selbst betont, dass die kognitiven Vorgänge im Fall der Imagination und mit Bezug zur realen Welt nicht identisch sind, sich aber zum Teil überschneiden. Er sagt: „I have emphasized the separation between our actual mental lives and the mental lives we lead in the worlds of our games of make-believe. But although they are distinct, there is substantial overlap between them“ (ebd., S. 252). Wenn emotionale und mentale Haltungen gegenüber fiktionalem Geschehen tatsächlich von denjenigen zu unterscheiden sind, die im Zuge realer Interaktionen entstehen zu unterscheiden sind, dann stellt sich die Frage, wie sie zu charakterisieren sind und wie sie entstehen. Eine Möglichkeit wäre, dass wir als Leser oder Teilnehmer eines Imaginationsspiels simulieren oder herleiten, wie wir uns als Beobachter einer vergleichbaren realen Situation fühlen. Damit wäre die eigene Emotion gegenüber der fiktiven Situation eine attribuierte Emotion. Ähnlich wie wir anderen im Rahmen realer Interaktionen mit Hilfe der ToM emotionale Vorgänge zuschreiben, können wir mit Hilfe der selben Mechanismen herleiten oder simulieren, wie wir in einer bestimmten Situation empfinden würden und uns diese emotionale Haltung selbst zuschreiben. Wenn das eigentliche instinktive Empfinden als Emotion erster Stufe beschrieben wird, dann würde es sich im Fall der Emotion einer fiktiven Situation gegenüber um eine Emotion zweiter Stufe handeln, die durch die voranstehende imaginative Kognitionsleistung geleitet wird.

\subsection{Imagination als kognitive Grundhaltung}

Timothy Schroeder und Carl Mathesons beschreiben die Imagination als eigenständige kognitive Grundhaltung bzw. als eigenständigen kognitiven Modus (,distinct cognitive atti$t u d e^{\natural}$ ), die von anderen kognitiven Haltungen (z. B. ,Überzeugung', ,Wunsch`, ,Intention` oder, Wahrnehmung ${ }^{6}$ ) zu unterscheiden ist. Wie alle anderen mentalen Haltungen kodieren kognitive Imaginationsakte einen bestimmten Inhalt (content). Dieser Inhalt besteht in einer 
Reihe von Informationen (tokens), die durch einen bestimmten Index gekennzeichnet werden. Dieser Index gibt an, dass die entsprechenden Informationen einer anderen Funktion im Rahmen der kognitiven Verarbeitung unterstehen, als die Inhalte anderer mentaler Haltungen (Schroeder \& Matheson, 2006, S. 23). Ein Mensch kann also den gleichen Inhalt mit Hilfe verschiedener mentaler Grundhaltungen repräsentieren. Ich kann mir in diesem Sinne wünschen, dass es regnet oder davon überzeugt sein, dass es regnet. Ich kann mir außerdem vorstellen, dass es regnet. Um diese Haltungen jeweils unterscheiden zu können - eine Fähigkeit die zum Kanon der ToM-relevanten Fähigkeiten gehört, muss der Inhalt der mentalen Haltung (,es regnet') kognitiv jeweils unterschiedlich indiziert sein. Eine solche Indizierung ermöglicht es auch, diesen Inhalt zeitglich im Rahmen mehrerer Grundhaltungen repräsentieren. Ich kann also davon überzeugt sein, dass es tatsächlich regnet, während ich mir gleichzeitig vorstelle, dass es regnet. Ich kann mir wünschen, Eiscreme zu essen, und mir gleichzeitig vorstellen, Eiscreme zu essen usw.

Schaun Nichols und Stephen P. Stich veranschaulichen diese Eigenschaft der menschlichen kognitiven Architektur mit Hilfe eines Modells aus unterschiedlichen ,Modulen', die semantische Informationen in jeweils adäquater Form kognitiv repräsentieren (Nichols \& Stich, 2003, S. 28 f.). So besitzen Menschen ihnen zufolge verschiedene Module, die Überzeugungen (belief-box), Wünsche (desire-box) und imaginative Inhalte (pretense-box) repräsentieren. Letzteres Modul der menschlichen kognitiven Architektur gestattet es uns, z. B. Dinge vorzugeben (pretend), uns Dinge vorzustellen (imagine) oder anzunehmen, etwas sei der Fall (suppose), was in Wirklichkeit nicht der Fall ist. Dies geschieht, ohne dass wir die Inhalte dieser Kognitionsakte mit dem Inhalt anderer ,Boxen` durcheinanderbringen. So kann ein Kind während des Fiktionsspiels vorgeben, dass es regnet, ohne davon überzeugt sein zu müssen, es regne tatsächlich.

Nichols zufolge können Informationen mit unterschiedlichen kognitiven Indices trotz der Zuordnung zu unterschiedlichen ,Boxen“ mit den gleichen kognitiven Verarbeitungsmechanismen prozessiert werden, da sie zwar unterschiedlich indiziert sind, sich gleichzeitig allerdings durch dieselbe kognitive ,Codierung، auszeichnen. In dieser Form stehen sie dem menschlichen kognitiven System, also u. a. den inferenzen- und emotionsbildenden Mechanismen, zur weiteren Prozessierung zur Verfügung. Anders gesagt: Wenn Überzeugungen (z. B. über einen bestimmten Sachverhalt der Wirklichkeit) und imaginative Inhalte mittels desselben kognitiven Codes prozessiert und anschließend für die Repräsentation der fiktiven 
Situation genutzt werden, dann können vergleichbare Inhalte, verbunden mit unterschiedlichen mentalen Haltungen, dennoch vergleichbare Inferenzen und Reaktionen hervorrufen (Nichols, 2004, S. 131). Diese Schlussfolgerung über die kognitive Verarbeitung imaginativer Gegenstände ist im Besonderen für die Rezeption darstellender Kunstwerke relevant. Ein Leser, der sich vorstellt, er beobachte Anna Karenina, die sich das Leben nimmt, bildet auf Grund der Parallelen im Kodierungsmechanismus auf demselben kognitiven Weg, d.h. mittels derselben kognitiven Mechanismen Inferenzen und emotionalen Reaktionen, als hätte er Anna Karenina tatsächlich dabei beobachtet, wie sie sich das Leben nimmt. Wenn dies zutrifft und die Inhalte von Überzeugungen wie Imaginationsakte durch die gleichen kognitiven Mechanismen verarbeitet werden, stellt sich die Frage, warum fiktionsbezogene und nicht-fiktionsbezogene Reaktionen des Lesers überhaupt zu unterscheiden sind. Wenn Emotionen einer fiktiven Situation gegenüber nicht mit denen realen Situationen gegenüber identisch sind, dann sind sie vielmehr Empfindungen ,zweiter Stufe ${ }^{‘}$, die wir generieren und uns selbst attribuieren indem wir uns vorstellen, eine fiktive Situation tatsächlich zu beobachten oder mitzuerleben. Ein wichtiger Unterschied zwischen der Fiktionsrezeption und der Interpretation realer Situationen besteht darin, dass - angenommen ein Leser nimmt eine adäquate Rezeptionshaltung gegenüber dem fiktionalen Text ein - die (imaginative) Vorstellung, Anna Karenina nehme sich das Leben, nicht mit der Überzeugung einhergeht, eine Person nehme sich tatsächlich das Leben. Allerdings kann ich mir vorstellen, davon überzeugt zu sein, eine Person (Anna Karenina) nehme sich wirklich das Leben. Darauf basierend wird eine angemessene emotionale Reaktion generiert. Ich möchte neben der ,singlecode'-Hypothese von Nichols noch einen weiteren Erklärungsansatz beleuchten.

\subsection{Eigene Erlebnisse und Empfindungen, denen ein Imaginationsakt voransteht}

Mit Blick auf Nichols Überlegungen stellt Alvin Goldman (2006a) die Frage:

Different boxes have different functional roles associated with them [...]. Now, functional roles are specified largely by dispositions to interact with other states and mechanisms in the larger cognitive system. Since pretense differs from belief in functional role, why should it be true that ,for any mechanism that takes input from both the pretense and the belief box, the pretense representation $p$ will be processed much the same way as the belief representation $p$ '? [...] why does sameness of code imply, or make probable, sameness of treatment? 
Ausgehend von der Annahme, dass Leser zumindest zu einem bestimmten Grad ihre Imagination einsetzen müssen, um sich die Ereignisse und Figuren, die in einem fiktionalen Text dargestellt sind, vorzustellen, nennt er den kognitiven Akt, der damit verbunden ist ,supposition-imagination“ (S-Imagination). S-Imagination beschreibt den Vorgang, wenn ein Leser in seiner Imagination evoziert, dass ein bestimmtes Ereignis stattfindet oder eine Figur eine bestimmte Handlung ausführt: „To s-imagine that $p$ is to entertain the hypothesis that $p$, to

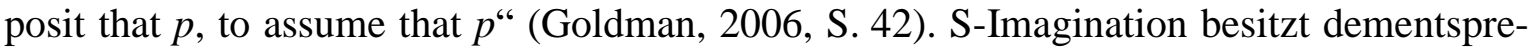
chend keine sensorische Dimension, sondern ist ein abstrakter, rein konzeptueller kognitiver Akt. Dem gegenüber stehen kognitive Vorgänge, die u. a. zur Generierung von Emotionen genutzt werden und die Goldman als ,enactment-imagination“ (E-Imagination) bezeichnet (ebd.). Während die S-Imagination als eine zusätzliche Kategorie kognitiver Grundhaltungen zu verstehen ist, trifft dies auf die E-Imagination nicht zu. Sie dient als Verarbeitungsmethode für die Inhalte der übergeordneten Kategorien mentaler Haltungen. Diese Verarbeitung von Informationen mit Hilfe der E-Imagination kann im Zusammenhang mit der Rezeption von Fiktion an Hand der jeweiligen Inhalte z. B. emotionale Reaktionen generieren:

Enactment-imagination is a matter of creating or trying to create in one's own mind a selected mental state, or at least a rough facsimile of such a state, through the faculty of imagination. Prime examples of E-imagination include sensory forms of imagination, where one creates, through imagination, perception-like states. (Ebd.)

Um dies zu erreichen, werden Inhalte, ähnlich wie von Nichols angenommen, mit Hilfe derselben emotionsgenerierenden Mechanismen verarbeitet wie im Zuge der Repräsentation realer Situationen. Goldman stützt seine Überlegungen mit einer Reihe empirischer Befunde, die zeigen, dass Leser während der Lektüre zum Teil die gleichen kognitiven Ressourcen für sensorische und motorische Aktivitäten nutzen, als würden sie die imaginierten Vorgänge tatsächlich ausführen. ${ }^{36}$ Diese Erkenntnisse sprechen für eine kognitive Simulation von literarisch vermittelten Ereignissen, die zu emotionalen Reaktionen führt, die von emotionalen Reaktionen gegenüber tatsächlichen Situationen schwer zu unterscheiden sind (Goldman, 2006b, S. 43). Mit Goldmans Worten: ,[E-imagination] can generate a state that resembles a counterpart perceptual state. Importantly, the imagined state resembles its perceptual counterpart not only in their shared code and content, but in a shared kind of state

\footnotetext{
${ }^{36}$ Für einen ausführlichen Überblick über die von Goldmann im Rahmen seiner Argumentation herangezogenen empirischen Daten vgl. Goldman (2006a) in den Kapiteln 2 und 7.
} 
[...]“، (ebd., S. 47). ${ }^{37}$ Um dies zu gewährleisten, muss Imagination die fiktionale Situation ,replizieren'bzw. ,simulieren'. Allerdings handelt es sich bei den resultierenden Produkten dieser Kognitionsprozesse um die Produkte einer bestimmten Form von Imagination und nicht um die Produkte der Verarbeitung von Informationen über die wirkliche Welt. Daher gesteht auch Goldman (ebd., S. 46) ein, dass fiktionsinduzierte emotionale Reaktionen nicht identisch mit emotionalen Reaktionen auf tatsächliche Ereignisse sind:

E-Imagination is a method or faculty that causes mental states of the various categories. Instead of causing them in a ,primary" way (each mental state type has its own distinctive cluster of primary causes), E-Imagination is a ,secondary' way of causing tokens of the same (or a very similar) type.

Simulationsprozesse dienen im Kontext realer sozialer Interaktion dazu, ,nachempfinden“ zu können, wie in bestimmten Situationen gedacht und empfunden wird. Sie dienen im Zuge der Rezeption fiktionaler Texte dazu, zu simulieren, wie in bestimmten Situationen gedacht und empfunden werden würde, wären diese Situationen real. Das bedeutet, dass zwar dieselben Mechanismen im Kontext der Lektüre eines fiktionalen Textes dazu genutzt werden, eigene Empfindungsprozesse zu generieren, wie im Rahmen realer Interaktionen. Die Grundlage, aufgrund dessen solche Empfindungen simuliert werden ist jedoch eine andere, nämlich eine Situation, die sich der Leser erst vorstellen muss. Leser sind dabei nicht nur mit der Aufgabe konfrontiert, die zu simulieren, wie fiktive Figuren denken und fühlen. Er muss außerdem eigene Empfindungen ,simulieren‘. Er empfindet, wie er empfinden würde, wären die fiktiven Geschehnisse wahr. Dies kann auf subjektiver Ebene zu einer täuschend Empfindungsreaktion führen, die von einer vergleichbaren gegenüber einer tatsächlichen Situation kaum zu unterscheiden ist. Kendall Walton schildert zur Unterstützung dieser Hypothese seine eigenen subjektiven Empfindungen während der Lektüre einer Textpassage, die eine Höhlenexpedition beschreibt. Durch die eigene Imagination provoziert - ohne tatsächlich an einer solchen Expedition teilzunehmen - beobachtet Walton bei sich selbst klaustrophobische Empfindungen (Walton, 1997). Dabei ist interessant, dass die von Walton zur Illustration gewählte Textpassage eine wichtige stilistische Besonderheit aufweist. Die Textpassage, auf die er sich bezieht, vermittelt die dargestellten Ereignisse in der zweiten Person Singular. Der Leser wird also - zumindest aus Sicht der gewählten grammatischen Form in direkter Form angesprochen: „You lower yourself into a hole in the ground and enter a dank, winding passageway [...]“ (ebd., S. 39). Auch in einem anderen Beispiel, das von

\footnotetext{
${ }^{37}$ Kursivierung durch den Autor.
} 
Walton mehrfach herangezogenen Beispiels findet ein in diesem Sinne vergleichbares stilistisches Mittel Verwendung. Gemeint ist der ,gefährliche Schleim', der den Kinobesucher Charles erschreckt. Hier verhält es sich so, dass mit den Mitteln der filmischen Umsetzung eine Interaktion zwischen der fiktiven Entität und dem Kinobesucher suggeriert wird. Charles erschrickt, ,[...] as the slime bore down on him from the screen“ (Walton, 1990, S. 241). In diesen besonderen Fällen sind Fiktionsrezipienten aufgrund der Beschaffenheit der entsprechenden Stimuli nicht nur aufgefordert, fiktive Szenarien nicht nur als unbeteiligte Beobachter zu imaginieren, sondern auch die Wirkung der entsprechenden Situationen auf sich selbst zu simulieren. In den von Walton gewählten Beispielen wird besonders deutlich, dass ein Leser oder ein Kinobesucher - ähnlich wie die Teilnehmer eines Fiktionsspiels - nicht notwendigerweise nur ein passiver Rezipient von Informationen ist; er wird von Walton vielmehr treffend mit den Worten beschrieben: „He is an actor, of a sort, in his game, as well as an object" (ebd., S. 242). Allerdings bleibt zu bedenken, dass Walton zur Illustration seiner These besondere Beispiele wählt, die aufgrund ihrer strukturellen und semantischen Eigenschaften die aktive mentale ,Beteiligung“ des Rezipienten möglicherweise erfordern und es bleibt zu klären, ob sich diese Hypothese auf die Fiktionsrezeption im Allgemeinen übertragen lässt.

Nichtsdestotrotz wird hier deutlich, dass die imaginative Auseinandersetzung eines Lesers mit den Inhalten des Fiktionsspiels oder eines darstellenden Kunstwerkes mit einer Kognitionsleistung einhergeht, die zum Kanon der ToM-relevanten Mechanismen gehört. Gemeint ist einerseits die Fähigkeit zur Simulation mentaler Haltungen und innerer Vorgänge, die sich in diesem Fall nicht auf die handelnden Figuren innerhalb der Fiktionswelt, sondern das eigene Selbst, das die fiktiven Geschehnisse in der eigenen Imagination mitverfolgt, bezieht. Die physiologischen Grundlagen dieses Mechanismus - wie z. B. die im ersten Teil der Arbeit näher beschriebenen Spiegelneuronen - sind in der Kognitionswissenschaft bereits zum Teil erforscht. Spiegelneuronen sind nachgewiesenermaßen nicht nur dann aktiv, wenn eine Handlung oder Teilhandlung (visuell) beobachtet wird, sondern auch dann, wenn typische mit einer bestimmten Handlung assoziierte Geräusche (auditiv) (Kohler, Keysers, Umiltà, Fogassi, Gallese \& Rizzolatti, 2002) verarbeitet, oder die entsprechenden Handlungen sprachlich beschrieben werden (Gallese, 2006, S. 663). Die durch Spiegelneuronen gestützten ,embodied simulation'- Prozesse sind also ebenfalls dann möglich, wenn Handlungen sprachlich (z. B. im Kontext der Lektüre eines literarischen Textes) vermittelt werden. Eine fMRI-Studie zeigt in diesem Zusammenhang: 
[...] action words referring to face, arm, or leg actions (e.g., to lick, pick, or kick), when presented in a passive reading task, differentially activated areas along the motor strip that either were directly adjacent to or overlapped with areas activated by actual movement of the tongue, fingers, or feet. These results demonstrate that the referential meaning of action words has a correlate in the somatotopic activation of motor and premotor cortex. (Hauk, Johnsrude \& Pulvermüller, 2004, S. 301)

Diese Ergebnisse legen nahe, dass das bloße Lesen über eine Handlung ausreicht, um dieselben Neuronengruppen zu aktivieren, die auch aktiviert würden, wenn eine Handlung in direkter Form (z. B. visuell oder akustisch) wahrgenommen wird. Dabei sind - wenn ausreichend Kontextinformationen zur Verfügung stehen - nicht nur diejenigen Neuronen involviert, die beobachtete Teilhandlungen ,spiegeln', sondern das Netzwerk derjenigen Zellen, die die (angenommene) vollständige Handlung repräsentieren (Fogassi, Ferrari, Gesierich, Rozzi, Chersi \& Rizzolatti, 2005). Indem Spiegelneuronen die neurophysiologische Grundlage dafür bilden, dass wir zukünftige zielführende Handlungsschritte antizipieren und damit z. B. motivierende Intentionen, Wünsche o. Ä. herleiten können, sind sie auch für die Interpretation textuell dargestellter Handlungen, nicht nur zum Erkennen bekannter Handlungsmuster, sondern auch zur Erschließung handlungsmotivierender mentaler Haltungen Empfindungen, relevant (Gallese, 2007, S. 662). Dies ist vor allem deshalb möglich, weil menschliches Handeln in literarischen Texten nicht nur abstrakt thematisiert, sondern vielmehr (schriftlich) dargestellt wird. Aufgrund dieses Merkmals sind Leser angehalten, nicht nur über bestimmte Handlungen nachzudenken, sondern sich die dargestellten Handlungen vorzustellen. Leser können literarisch dargestellte Handlungen zwar nicht in direkter Form beobachten, wohl aber kognitiv rekreieren, indem sie z. B. eine Abfolge von entsprechenden kognitiven Situationsmodellen bilden. Damit besteht trotz des differenten Kognitionsmodus die Möglichkeit, diese Handlungen mit Hilfe derselben kognitiven Mechanismen (in diesem Fall, der Spiegelneuronen) auszuwerten.

Ich möchte nach diesen Überlegungen festhalten, dass Menschen im Zuge der Fiktionsrezeption. Wenn sich ein Museumsbesucher bspw. Willem Van der Velde's Shore at Sheveningen ansieht und sich vorstellt, am Strand zu stehen und eine Reihe von Schiffen zu betrachten können bestimmte kognitive Mechanismen der ToM (u. a. Spiegelneuronen) dafür eingesetzt werden, fremde (fiktive) aber auch eigene Empfindungen zu simulieren. Ob und in welchem Ausmaß einzelne Werke dies einem Fiktionsrezipienten gestatten bzw. von ihm einfordern, bleibt an Hand der individuellen Beschaffenheit dieser Werke zu klären. 


\section{Die Methode der kognitivistischen Textanalyse}

Die Überlegungen dieses zweiten Hauptteils der Dissertationsschrift galten bisher der kognitiven Rezeption typischer literarischer Erzähltexte. Unter einem literarischen Erzähltext verstehe ich - in groben Zügen - einen Text, der sich durch die Merkmale der Narrativität, Fiktionalität und Literarizität auszeichnet. Literarische Texte enthalten außerdem eine auf bestimmte Art und Weise strukturierte Menge von Informationen, die im Zuge ihrer Rezeption kognitiv ausgewertet werden müssen. Während Leser die geschilderten Situationen sukzessive kognitiv modellieren, entsteht nach und nach eine Repräsentation der geschilderten Welt - das Fiktionsweltmodell. Dieses Fiktionsweltmodell besitzt eigene deiktische Bezugspunkte, so dass Objekte, Orte, Personen, ihre Wahrnehmung und Handlungen sowie die Wahrnehmung und das Empfinden des Lesers mit Bezug zu diesen fiktionseigenen Bezugspunkten interpretiert werden können. Das bedeutet weiterhin, dass Autoren ihre Leser regelrecht dazu ,anleiten“ können, zu einem bestimmten Zeitpunkt während der Lektüre eine bestimmte Situation aus einer bestimmten Perspektive zu repräsentieren. Auf diese Weise entsteht mit den durch den Autor vorgegebenen und den Leser rekonstruierten Situationsmodellen ein gemeinsamer Referenzrahmen.

Ich Überlegungen wie diese einleitend zu dieser Arbeit der Gruppe text-zentrierter Hypothesen zur Relation zwischen Textmerkmal und Leserkognition zugeordnet. Hier richtet sich die Aufmerksamkeit auf die ,bottom-up'-orientierte Verarbeitung von Informationen, wobei die spezifischen Charakteristika des literarischen Textes von entscheidender Bedeutung sind. Es gilt die grundsätzliche Annahme, dass sich das literarische Werk als ,Anleitung` zur kognitiven Rekonstruktion von einer Reihe von Ereignissen lesen lässt. Der Text selbst bildet hier die von einem (oder mehreren) Autor(en) geschaffene Grundlage für die Bildung eines gemeinsamen kognitiven Referenzrahmens. Der kognitive Mechanismus, der es zwei Menschen (in diesem Fall Autor und Leser) gestattet, einen solchen gemeinsamen Referenzrahmen für kommunikative Prozesse zu schaffen, entwickelt sich dann, wenn Kinder im realen sozialen Kontext beginnen die eigene Aufmerksamkeit an derjenigen einer Bezugsperson auszurichten. Sie zeigen hier bereits die Fähigkeit, impliziten und expliziten Kommunikationssignalen Folge zu leisten und die eigene Kognition entsprechend anzupassen. Mit dieser Fähigkeit ist die Grundlage gelegt, sprachlichen Kommunikationssignalen Folge zu leisten, ,unter Anleitung' die Wahrnehmung auf ein bestimmtes Geschehen zu lenken und das Geschehen aus einer kognitiv rekonstruierten Perspektive zu repräsentieren. Das Kommunikationsmedium ist im Fall der Lektüre eines literarischen Erzähltextes (wie an anderer 
Stelle bereits ausgeführt) nicht die reale soziale Interaktion, in der kommunikative Gesten (wie z. B. Zeige- oder Blickgesten) genutzt werden können, sondern der literarische Text (Luther, 2006b). Die in ihm in strukturierter Form zusammengestellten Informationen bilden den Ausgangspunkt für die Konstruktion eines gemeinsamen kognitiven Referenzrahmens für die Imagination einer bestimmten Ereignisfolge von bestimmten Personen und Objekten (ebd., S. 182 f.). Über die bewusste Gestaltung des Referenzrahmens hat ein Autor die Möglichkeit, die kognitive Rezeption des Lesers in gewissem Ausmaß zu lenken und ihm die Art und den Grad des Realitätsbezuges einer Aussage anzuzeigen oder ihn darüber zu täuschen. Die ,mentale‘ Anleitung durch einen Autor beschränkt sich in diesem Zusammenhang nicht ausschließlich auf den Realitätsbezug der Aussagen in einem literarischen Text.

Im Zuge einer kognitivistischen Textanalyse liegt der Fokus darauf, diese ,mentale“ Interaktion zwischen Autor und Rezipient über das Medium des Textes soweit wie möglich zu rekonstruieren. Ich habe zuvor in dieser Arbeit bereits vorgestellt, dass die deiktischen Feinstrukturen einer Textpassage Auskunft über die kognitive Rekonstruktion von Perspektivität während der Lektüre dieser Passage geben können. An dieser Stelle habe ich bereits angedeutet, dass sich Textpassagen daraufhin untersuchen lassen, welche Bedingungen ihre strukturelle und semantische Beschaffenheit für die kognitive Rezeption durch einen Leser stellt. Um einschätzen zu können, welche kognitiven Prozesse während der Lektüre eines literarischen Textes ablaufen, ist allerdings eine umfassendere Analyse seiner Semantik- und Strukturmerkmale notwendig, die dann mit den bekannten Mechanismen der menschlichen Kognition in Verbindung gesetzt werden müssen. Hierfür ist es notwendig nicht nur die Mechanismen der menschlichen Kognition (in dieser Arbeit mit besonderem Fokus auf den Mechanismen der ToM) und ihre Funktionen zu kennen, sondern auch die individuellen Textmerkmale möglichst präzise benennen zu können. Hierfür ist mit Blick auf die konkrete, praktische Textanalyse vor allem das Instrumentarium narratologischer Begriffskonzepte hilfreich. In der Narratologie wurden in der Vergangenheit eine Reihe von Taxonomien zur Klassifikation typischer narrativer Merkmale entwickelt und diskutiert. Gérard Genettes erzähltheoretischer Ansatz, auf den ich in dieser Arbeit Bezug nehme, ist nur einer von mehreren, die in diesem Zusammenhang zu nennen sind. Mit Hilfe solcher Begriffe lassen sich (ähnlich wie auch im vorangehenden Abschnitt dieser Arbeit mit Blick auf die kognitive Ereignisrekonstruktion) Überlegungen über die Bedingungen für die kognitive Informati- 
onsverarbeitung im Zusammenhang mit typischen narrativen Situationen anstellen. Das Beispiel der unterschiedlichen typischen Perspektivierungsstrategien nach Gérard Genette soll dies besser veranschaulichen. ${ }^{38}$

Eine erste solche grundlegende Perspektivierungsstrategie ist laut Genette die externe Perspektive. Im Fall einer externen Perspektive steht die reine Handlungsschilderung im Vordergrund, ,[...] ohne daß uns je Einblick in [die] Gedanken und Gefühle [des Helden] gewährt würde“ (Genette, 2010, S. 121). ${ }^{39}$ Martinez und Scheffel (2007, S. 64) definieren diese Erzählhaltung als ,Außensicht‘, wobei „der Erzähler [...] weniger [sagt], als die Figur weiß.“ Diese Situation bringt es mit sich, dass die ,Sicht' auf die Ereignisse und Handlungen innerhalb der Fiktionswelt unverstellt ist, jedoch kein direkter Informationszugang zu den inneren Haltungen und Vorgängen der Figuren besteht. Dieser Informationssituation entsprechend muss der Leser an den Stellen, an denen es für ein kohärentes Textverständnis notwendig ist, entsprechende Schlussfolgerungen eigenständig ziehen. Wenn keine direkten Informationen über innere Vorgänge vermittelt werden, bleibt ihm in diesem Fall die Möglichkeit, Hinweisen über, sichtbare‘ Vorgänge (z. B. der Beschreibung mimischer oder gestischer Vorgänge) zu folgen oder einzelne Ereignisse unter Berücksichtigung bestimmter (z. B. kausallogischer) Regeln miteinander zu verknüpfen. Diese Kognitionsleistung ist derjenigen innerhalb realer sozialer Kontexte ähnlich, weil auch hier ein egozentrisch orientierter Blick von außen auf die an einer sozialen Interaktion beteiligten Personen ausschlaggebend ist, um die Handlungen anderer zu interpretieren. Um die ,Sichtweise“ einer handelnden Person (im realen sozialen Kontext) oder einer fiktiven Figur (im Kontext der Fiktionswelt) interpretieren zu können, müssen Leser lediglich alternative subjektgebundene Modelle der jeweiligen Umgebung oder Situation konstruieren und der entsprechenden Figur zuschreiben. Da sich eine bestimmte innere Disposition in den ,beobachtbaren` Handlungen einer Person (im realen Kontext) oder einer Figur (im fiktionalen Kontext) niederschlagen kann, lassen sich in beiden Fällen mittels des Wissens über die sichtbaren bzw. beschriebenen Handlungen Rückschlüsse auf mentale Haltungen ziehen und vice versa.

\footnotetext{
${ }^{38}$ Welche Begriffe, beziehungsweise welche narratologischen Modelle zur Beschreibung von Textmerkmalen im Zuge einer kognitivistischen Textanalyse Anwendung finden, sollte mit Blick auf ihre Anwendbarkeit mit Blick auf den individuellen Text und das Analyseziel entschieden werden. Es ist dabei wichtig, ein bestimmtes Textmerkmal so genau und treffend wie möglich zu identifizieren. Die Wahl der begrifflichen Taxonomie würde in diesem Fall gegenüber der Präzision der Merkmalsbeschreibung in den Hintergrund treten. Auf der anderen Seite ist es aber auch möglich, einzelne Texte hinsichtlich ihrer kognitiven Leistungsanforderungen miteinander zu vergleichen. In diesem Fall wäre es ggf. sinnvoll ein Raster vergleichbarer Begriffskategorien anzulegen.

${ }^{39}$ Hervorhebungen des Autors.
} 
Anders verhält es sich mit Situationen, in denen Ereignisse aus einer inneren Perspektive geschildert werden. In Passagen, die einer ,inneren“ Perspektivierungsstrategie folgen, ist die Darstellung der Ereignisse an den subjektiven ,Blickwinkel` einer bestimmten Instanz (zum Beispiel einer der fiktiven Figuren) gebunden. Die Ereignisse werden mit einem Blick ,über die Schulter“ oder sogar ,durch die Augen` einer bestimmten Figur vermittelt. Dementsprechend erhalten Leser lediglich zu Ereignissen und Zusammenhängen Zugang, von denen die jeweilige Figur berichten kann. Der Wissenszugang des Rezipienten gleicht in diesem Fall demjenigen der Figur bzw. unterliegt den entsprechend festgelegten Beschränkungen. Das führt dazu, dass die kognitive Rekonstruktion des Fiktionsweltmodells auf zwei Ebenen abläuft. Eine interne Perspektive bringt es mit sich, dass der Blick auf die Ereignisse von der Vorinterpretation/Gefühlslage/Intention der - mit den Begriffen von Genette gesprochen - ,fokalisierenden' Figur abhängig ist. Wayne C. Booth (1961, S. 151) schreibt: „most tales are presented as passing through the consciousness of a teller, whether an "I" or a "he" $[\ldots]$ and we are often as much interested in the effect on the narrator's own mind and heart [...]." Da im Fall einer internen Perspektive, wie hier von Booth geschildert, die Ereignisse nicht nüchtern präsentiert, sondern durch die ,subjektive“ Wahrnehmung einer fiktiven Instanz gefiltert und in dieser Form dem Rezipienten vor Augen geführt werden, entsteht die Notwendigkeit, die Informationen durch eine entsprechende Interpretationsleistung zu evaluieren und ggf. zu relativieren. Der kognitive Vorgang der Relativierung ist dann für die Interpretation narrativer Informationen notwendig, wenn die Figur aufgrund ihres Wissens, ihrer Überzeugungen, ihrer Wesensmerkmale und Wünsche die ,fiktive Realität ${ }^{\star}$ anders repräsentiert als der Leser oder (z. B. im Fall einer Täuschungsabsicht) willentlich ein anderes ,Bild‘ der Fiktionswelt zeichnet. Zusätzlich können literarische Texte so strukturiert sein, dass sie nicht nur die kognitive Rekonstruktion der ,Sichtweise“ einer einzigen Figur auf die geschilderten Ereignisse erforderlich ist. Durch eine entsprechende Konfiguration des Textes kann ebenfalls initiiert werden, dass die interne Perspektive im Verlauf der fortschreitenden Textlektüre variabel zwischen mehreren Figuren wechselt und daher entsprechend viele ,Blickwinkel‘ oder fiktive Wahrnehmungskontexte nachvollzogen werden müssen, um bestimmte Textpassagen korrekt zu verstehen. Entsprechend müssen Leser an solchen Stellen ein Fiktionsweltmodell erstellen, das die ,tatsächliche' Situation innerhalb der Fiktiven Welt abbildet, und gleichzeitig so viele perspektiv-gebundene Modelle (als Teil der attribuierten kognitiven Kontexte der jeweiligen Figuren) konstruieren, wie es der Text vorgibt. 
Eine dritte typische Erzählstrategie, die an dieser Stelle nicht vergessen werden darf, ist - in Genettes Terminologie - die Nullfokalisierung. Im Fall einer ,Null'-Fokalisierung werden die Geschehnisse durch eine Erzählstimme geschildert, die eine übergeordnete Stellung zum Geschehen einnimmt. Dabei handelt es sich um eine abstrakte Instanz, deren ,Gesichtsfeld“ aufgrund der übergeordneten Stellung nicht oder nur kaum eingeschränkt ist (Genette, 2010, S. 248). Im Regelfall weiß ein Leser, dank diesem Blick, von oben', mehr über das Geschehen als die einzelnen Figuren, die an dem Geschehen teilhaben. Dennoch können Autoren ihren Lesern auch in diesem Fall interpretationsrelevante Informationen vorenthalten. Wichtig ist, dass im Fall der Nullfokalisierung keine Erzähl-, figur' konfiguriert wird, deren spezifisches Profil, Denken und Handeln Leser bei der Interpretation der gegebenen Informationen berücksichtigen müssten. Die übergeordnete und nicht an eine bestimmte fiktive Figur oder Erzählinstanz gebundene Erzählsituation in Texten mit Nullfokalisierung gestattet Lesern außerdem scheinbar ,direkte' und unvermittelte Einblicke in die Welt der Gedanken, Emotionen und mentalen Haltungen der unterschiedlichen Figuren. Ein solcher, ,objektiver ${ }^{6}$ Zugang zu inneren Vorgängen markiert einen deutlichen Unterschied zum realen sozialen Kontext in dem solche Vorgänge entweder durch das betroffene Subjekt referiert oder durch einen Beobachter attribuiert werden müssen. Vermittelt durch die körperlose Erzählstimme stehen somit potenziell auch Informationen über die mentalen Vorgänge der Charaktere zur Verfügung, die er nicht im Zuge des Interpretationsprozesses selbst attribuieren muss.

Neben den Perspektivierungsstrategien einer Passage ist auch die Stellung der Erzählinstanz zum geschilderten Geschehen relevant. Um die möglichen Modellierungen der räumlichen Erzählposition begrifflich zu erfassen, definiert Genette zwei typische Situationen, „,solche in denen der Erzähler in der Geschichte, die er erzählt, nicht vorkommt, abwesend ist [...] und solche in denen der Erzähler als Figur in der Geschichte, die er erzählt, anwesend ist [...] (ebd., S. 159) Er nennt den ersten einen heterodiegetischen Erzähler und den zweiten einen homodiegetischen Erzähler. Genette weist zusätzlich darauf hin, dass die Abwesenheit eines Erzählers immer absolut ist, während seine Anwesenheit und Teilnahme am Handlungsgeschehen graduellen Abstufungen unterliegen kann. Ein oberer Grenzwert dieser Skala ist mit der sogenannten autodiegetischen Erzählsituation erreicht, in welcher der Erzähler und die Hauptfigur des Geschehens in einer Person vereint sind (ebd.).

Der Einfluss der narrativen Position auf die für ein gelingendes Textverständnis notwendigen Kognitionsleistungen des Lesers unterscheidet sich im Vergleich der beiden Grundsitu- 
ationen beträchtlich. Zwei Faktoren spielen hierbei eine Rolle. Der erste dieser beiden Faktoren ist die der Unterschied zwischen einem Erzählsubjekt, das an den Geschehnissen, von denen es berichtet, selbst teilnimmt, und einer Erzählinstanz, die außerhalb des Geschehens steht. Im ersten Fall müssen Leser das ,Profil‘ der erzählenden Figur kontinuierlich aktualisieren, da sie Teil der Fiktionswelt und daher von den erzählten Ereignissen möglicherweise selbst betroffen ist. Ein heterodiegetischer Erzähler steht in diesem Sinne außerhalb des Geschehens und ist von den Geschehnissen innerhalb des Handlungskontinuums nicht unmittelbar betroffen. Er ist lediglich eine berichtende und möglicherweise reflektierende Instanz und muss dementsprechend nicht als Figur in das entsprechende Situationsmodell integriert werden. Ein homodiegetischer Erzähler nimmt hingegen in direkter Form an der Situation teil und Leser müssen solche Instanzen entsprechend in das Fiktionsweltmodell integrieren. In Abhängig von ihrer Erzählposition kann die Qualität der Informationen, die dem Leser über die Erzählinstanz selbst und über die geschilderten Ereignisse zur Verfügung stehen, stark variieren. Im Fall eines heterogetischen Erzählers sind bestimmte Informationen, die Leser z. B. anhand von Beschreibungen seiner Interaktion mit anderen Figuren beziehen könnte, nicht gegeben. Handelt es sich um einen Erzähler, der Teil des Geschehens ist, stehen dahingegen Informationen zur Verfügung, die sich aus den Reflexionen, Beschreibungen und Reaktionen anderer Figuren und den geschilderten Handlungen des erzählenden Subjektes selbst speisen, um ein adäquates ,mentales Profil‘ zu erstellen. Allerdings ist auch hier ein großer Spielräume für die Dichte der vermittelten Informationen möglich. Um in diesem Fall begründete Aussagen über die Kognitionsleistung des Lesers zu formulieren, ist es notwendig, die individuelle Konfiguration einzelner Werke genauer zu analysieren. Nur an Hand der gegebenen Strukturen des Einzeltextes lässt sich nachvollziehen, unter welchen Bedingungen die kognitive Rezeption im Einzelnen abläuft und inwiefern die Stellung der Erzählinstanz zum erzählten Geschehen einen Faktor im Rahmen dieser Bedingungen darstellt. Die Textanalysen im dritten Teil dieser Arbeit bieten Raum für solche Untersuchungen.

Die Beispiele unterschiedlicher narrativer Perspektivierungsstrategien und der Positionierung des Erzählsubjekts zeigen, dass die Wahl der Erzählstrategie und damit die Beschaffenheit der Informationsvermittlung Einfluss auf die Art der kognitiven Interpretationsleistung des Rezipienten nimmt. Es entsteht in diesem Fall ein Abhängigkeitsverhältnis zwischen dem Zugang zu interpretationsrelevanten Informationen und der kognitiven Evaluation dieser Informationen, die zur Erstellung einer adäquaten kognitiven Repräsentation der 
Fiktionswelt beitragen. Um dieses Abhängigkeitsverhältnis genau zu erfassen und zu beschreiben, müssen die spezifischen Strukturen eines Textes analysiert werden. Narratologische Begriffe bieten in diesem Fall ein Mittel zur Orientierung. Ihre Funktion zur Einordnung bestimmter typischer Strukturen in Kategorien birgt jedoch die Gefahr, die feineren für die kognitive Rezeption jedoch relevanten - Strukturen zu übersehen. Es ist also durchaus möglich, dass sich die kognitiven Rezeptionsprozesse bestimmter Texte nicht oder nur schwer mit den gegebenen Begriffen erfassen lassen.

Im dritten Teil dieser Arbeit möchte ich mich - ausgehend von diesen Beobachtungen neben den typischen und individuellen Perspektivierungsstrategien auf eine breitere Menge von relevanten Textmerkmalen beziehen. Es wird interessant sein, zu beobachten, inwiefern die Art und Menge der Informationen, die durch einen literarischen Erzähltext vermittelt werden, von der Art und Menge der Informationen in gewöhnlichen realen sozialen Situationen abweicht und die besondere Beschaffenheit eines einzelnen Textes die Kognitionsmechanismen seiner Leser stimuliert.

\section{$7 \quad$ Fazit}

Die ToM gestatten es uns Menschen, viele unterschiedliche soziale Interaktionssituationen zu meistern. Der Grund dafür ist, dass uns dank ihr ein Verständnis für die Inhalte und Funktionen fremder wie eigener innerer Vorgänge und mentaler Haltungen gegeben ist. Die Vielzahl unterschiedlicher, mit der ToM verbundenen kognitiven Leistungen lassen sich $\mathrm{m}$. E. nur durch das Zusammenwirken einzelner kognitiver Mechanismen erklären. Die Fähigkeit zur kognitiven Repräsentation fremder mentaler Kontexte und die Wahrnehmung, Organisation und Verarbeitung der hierfür notwendigen Informationen basieren nicht auf einer einzelnen kognitiven Strategie (Theorie vs. Simulation) oder einem einzelnen kognitiven Mechanismus. Sie werden vielmehr durch ein komplexes System verschiedener ineinandergreifender kognitiver Mechanismen ermöglicht, die simulative, abstrahierende, metakognitive und integrative Kognitionsprozesse miteinander verbinden.

Wie die ToM gehört auch die Fähigkeit zur literarischen Textrezeption zu den besonderen kognitiven Fähigkeiten des Menschen. Eine der zentralen Aufgaben bestand hier darin, herauszufinden, ob es einen Zusammenhang zwischen diesen zwei besonderen kognitiven Kompetenzen gibt und wie dieser Zusammenhang zu charakterisieren ist. Um dies zu klären, 
wurde im vorangehenden zweiten Hauptteil dieser Arbeit nach einem möglichen kognitiven ,common ground‘ gesucht. Dabei galt es zu prüfen, ob sich fundierte Argumente finden lassen, die die von Lisa Zunshine (2006; 2010) und Alan Palmer (2004) formulierten Thesen stützen. Die Frage lautete, warum und und auf welche Weise Menschen diejenigen Mechanismen, die ihre ToM ermöglichen, analog zum sozialen Kontext auch für die Interpretation literarisch vermittelter Handlungen nutzen. Diese Frage ließ sich mit einem Blick auf grundlegende Prozesse der kognitiven Informationsverarbeitung bejahen. Gestützt wird dieses Fazit durch die Annahme, dass sowohl die ToM als auch die literarische Textrezeption durch ein besonderes Prinzip der menschlichen Kognition gestützt werden. Gemeint ist die kognitive Modellierung von realen, fiktiven oder hypothetischen Situationen. Obwohl die Informationsstrukturen, die uns Menschen jeweils im Kontext realer Interaktionen und im Kontext der literarischen Textrezeption zur Verfügung steht, unterschiedlich sind, sind doch in beiden Fällen die notwendigen Voraussetzungen dafür gegeben, dass im Zuge der Informationsverarbeitung kognitive Modelle entstehen können. Also ist in beiden Fällen eine vergleichbare kognitive Grundlage für weiterführende Inferenzen und Interpretationsvorgänge geschaffen. Damit ist eine Erklärung für die intuitive Annahme gefunden, dass Menschen ihre ToM im Zuge der Textlektüre gegenüber Autoren und fiktiven Figuren ebenso für die Rekonstruktion motivationaler Zusammenhänge einsetzen können, wie in der realen Interaktion.

Darüber hinaus lautete meine initiale Hypothese, dass die kognitiven Leistungen der ToM auf einer grundlegenden Ebene in die kognitive Rezeption literarischer Erzähltexte eingebunden ist. Ihre Rolle geht über die Attribution mentaler Haltungen zugunsten eines kohärenten Handlungsverständnisses hinaus. In den einzelnen Abschnitten in diesem Kapitel habe ich die unterschiedlichen Leistungsanforderungen, die durch typische Merkmale literarischer Erzähltexte entstehen, mit denjenigen der ToM verglichen. Dabei waren auf unterschiedlichen Ebenen der literarischen Rezeption strukturelle Parallelen in der kognitiven Informationsverarbeitung zu finden. Die im ersten Teil der Arbeit geleistete detailliertere Aufschlüsselung der einzelnen ToM-Mechanismen gestattete es, neben der durch einen Leser geleisteten Attribution mentaler Haltungen gegenüber Autoren und fiktiven Figuren weitere parallele kognitive Leistungsanforderungen zwischen typischen ToM-Aufgaben und der literarischen Rezeption zu benennen. Ich möchte an dieser Stelle zusammenfassend die Erkenntnisse dieses zweiten Arbeitsteils festhalten: 
1. Die Fähigkeit zur Attribution von Intentionen ist nicht nur für die Interpretation psychologisch motivierter Handlungen auf der Ebene von Handlungen und Ereignissen relevant, die in literarischen Erzähltexten dargestellt sind. Sie gestattet Autoren und Leser darüber hinaus auch, über Kommunikationssignale einen ,mentalen Austausch` miteinander. Dieser ist vor allem dann relevant, wenn Leser einen geeigneten kognitiven Rezeptionsmodus gegenüber einem fiktionalen Werk oder einer Aussage, die in einem bestimmten Verhältnis zur Realität steht (z. B. fiktiv ist), einnehmen müssen, um sie richtig zu verstehen. Autoren können ihre Leser durch die Gestaltung der Aussagen im Text dazu anleiten, eine bestimmte Rezeptionshaltung einzunehmen. Leser können sich an diesen Hinweisen orientieren und entsprechende Aussageintentionen attribuieren. Autoren können im Gegenzug die Rezeptionshaltung ihrer Leser antizipieren und dies bei der Gestaltung der Informationen, die sie zur Verfügung stellen, wenn sie einen Text verfassen, berücksichtigen.

2. Leser müssen im Zuge der Textlektüre an Hand deiktischer Hinweise Wahrnehmungsstandpunkte und Perspektiven innerhalb des deiktischen Bezugssystems der Fiktionswelt konstruieren und repräsentieren. Es ist für ein gelingendes Textverständnis ausschlaggebend, diese Perspektiven bei der Auswertung und Interpretation der Informationen zu berücksichtigen. Diese kognitive Leistung findet ihr Pendant im realen sozialen Kontext in der Fähigkeit zur Rekonstruktion fremder Perspektiven und Wahrnehmungskontexte. Die deiktischen Strukturen eines Textes funktionieren in diesem Sinne wie ,Fingerzeige', die Leser anleiten, die Fiktionswelt aus einem bestimmten ,Blickwinkel ${ }^{\star}$ $\mathrm{zu}$ betrachten und entsprechend zu rekonstruieren.

3. Ereignisse, die in einem Erzähltext vermittelt werden, stehen der direkten Wahrnehmung durch die Sinnesorgane nicht offen. Sie werden vielmehr durch einen narrativen Akt , vermittelt ${ }^{\star}$, so dass in jedem Fall eine eingebettete Informationsvermittlungsstruktur entsteht, die sich durch Komplementsätze ausdrücken lässt (E sagt, X sei der Fall/ Im Text steht, X sei der Fall). Um solche Informationen verarbeiten zu können, ist ein kognitiver Mechanismus erforderlich, der es gestattet, die Ebenen des Gesagten und des Erzählvorgangs in die richtige Beziehung zueinander zu stellen. Vor allem mit Blick auf die Korrelationen von ToM-Leistungen und grammatisch-syntaktischen Fähigkeiten ist es denkbar, dass hier diejenigen kognitiven Ressourcen aktiviert werden, die u. a. auch dafür zuständig sind, Inhalt und Funktion von bestimmten mentalen Haltungen in Abhängigkeit voneinander zu entschlüsseln (A denkt, X sei der Fall vs. A weiß, X sei der 
Fall vs. A möchte, dass X usw.). Auch wenn in einem Fall ein bestimmter Inhalt (X) in den Rahmen der Informationsvermittlung bzw. im anderen Fall in Abhängigkeit von einer bestimmten Kategorie mentaler Haltungen eingebettet ist, so ist es doch in beiden Fällen notwendig eine Relation zwischen beiden Ebenen herzustellen, um die gegebenen Informationen korrekt auswerten zu können.

4. Das Informationssystem eines literarischen Textes kann bestimmte Merkmale enthalten, die den Text selbst als einen literarischen kennzeichnen. Da Menschen neben den Inhalten einer Darstellung oder Situation im Zuge der Informationsverarbeitungsprozesse auch meta-modale Informationen über die Art, Struktur und Wahrnehmungsform der zur Verfügung stehenden Daten prozessieren können, ist es wahrscheinlich, dass sie aufgrund von bestimmten Literarizitäts-Kriterien Informationen über die formalen, strukturellen und rezeptionsspezifischen Besonderheiten (z. B. das verwendete Sprachsystem) oder ästhetische Eigenschaften des Textes kognitiv kodieren. Neben einfachen metalinguistischen Informationen können im Fall von literarischen Erzähltexten, die nicht nur sprachlich vermittelt sind, sondern ihre Darstellungsmodi ggf. durch bestimmte Mittel in den Vordergrund stellen, auch meta-poetische Repräsentationen in kognitive Rezeption einfließen. Das ist vor allem dann wahrscheinlich, wenn die linguistischen Eigenschaften von Texten deutlich von den gewohnten Sprachsystemen, z. B. demjenigen der Alltagssprache, abweichen. Darüber hinaus ist denkbar, dass im Fall von spezifischen Gattungen literarischer Texte nicht nur Informationen über die besondere Poetizität der Sprache, sondern auch Informationen über weitere Merkmale, die diese Texte auszeichnen, kodiert werden. Wenn sich diese Eigenschaften als Teil der Vermittlungsmodalität in einem entsprechenden mentalen Modell wiederfinden, dann lässt sich über kognitive Fiktionsweltmodelle sagen, dass sie sowohl aus primären, als auch aus sekundären und Meta-Repräsentationen bestehen. Mit anderen Worten: Es gehört zum Prozess der literarischen Rezeption, nicht nur die dargestellten Ereignisse und Figuren, sondern auch Informationen über die dargestellten Ereignisse und Figuren, die Darstellungsmodalität sowie über den literarischen und fiktionalen Charakter der Informationsvermittlung kognitiv zu kodieren. Wie im ersten Teil der Arbeit näher erläutert, ist die Bildung von Repräsentationen unterschiedlicher Abstufung auch für verschiedene Dimensionen der ToM (z. B. das Verstehen der Funktion verschiedener Kategorien mentaler Haltungen, das Verstehen von hypothetischen, fiktiven, simulierten Szenarien im Zuge des Fiktionsspiels etc.) eine zentrale Voraussetzung. 
5. Im Zuge der Lektüre fiktionaler Texte stellen sich Leser der Aufgabe, die dargestellten Ereignisse außerdem mit Hilfe der eigenen imaginativen Fähigkeiten vorzustellen. Die Ergebnisse der in diesem Teil erwähnten vorangehenden Arbeiten und die darauf aufbauenden Überlegungen führen zu dem Schluss, dass ein Teil des Rezeptionsaktes als Simulation eigener Erfahrungen und Empfindungen gegenüber einer dargestellten (fiktiven) Situation ist. Leser modellieren dabei nicht nur einen eigenen ,Standpunkt“ als „Beobachter' oder, wie in Waltons Beispiel, ,Teilnehmer‘ der Fiktionswelt. Sie können dabei das gesamte Repertoire der relevanten ToM-Mechanismen nutzen, um mit Hilfe der eigenen Imagination zu repräsentieren, wie es sich anfühlen würde, Beobachter oder Teilnehmer der dargestellten Situation zu sein.

Mein Fazit lautet mit Blick auf die genannten Punkte, dass die Lektüre literarischer Erzähltexte trotz aller Differenzen zum realen sozialen Kontext eine Reihe kognitiver Mechanismen aktiviert, ohne die auch die ToM nicht möglich wäre. Diese Mechanismen betreffen zentrale Dimensionen der literarischen Rezeption und sind mit wichtigen Merkmalen wie z. B. der Fiktionalität, Narrativität und Literarizität des Textes verbunden. Da sie durch wichtige oder sogar konstitutive Merkmale literarischer Erzähltexte stimuliert bzw. durch sie für ein gelingendes Textverstehen vorausgesetzt sind, lautet mein Schluss, dass die Rezeption literarischer Erzähltexte so, wie wir sie gewohnt sind, ohne die grundlegenden Mechanismen der ToM nicht möglich wäre. Die Interpretation psychologisch motivierter Handlungen und das Verstehen von fiktiven Handlungen, in denen die ToM der Figuren ein wichtiges Element darstellt, ist dabei nur eine von mehreren ToM-relevanten kognitiven Leistungen, die Leser im Zuge der von literarischen Erzähltexten erbringen müssen. Die beiden Fähigkeiten der ToM und der literarischen Textrezeption beruhen vielmehr zu einem großen Teil auf denselben kognitiven Mechanismen. Vittorio Gallese (2008, S. 181) formulierte hinsichtlich der hirnphysiologischen Prozesse des prämotorischen und motorischen Kortex und ihre Rolle für das menschliche Handlungsverständnis die ,neural exploitation'-Hypothese. Er schreibt:

My main claim is that the key aspects of human social cognition are produced by neural exploitation, that is, by the adaptation of neural mechanisms originally evolved for sensory-motor integration, later on also employed to contribute to the neurofunctional architecture of thought and language, while retaining their original functions as well. 
Auch meine Argumentation, die auf der funktionalen Beschreibung der ToM, mit Hilfe der für sie relevanten Mechanismen, und einem Vergleich der Leistungsanforderungen in unterschiedlichen Verstehenskontexten basiert, kommt trotz des unterschiedlichen methodischen Ansatzes zu demselben Schluss. Er lautet, dass die auf den ersten Blick so unterschiedlichen Fähigkeiten des Menschen (einerseits die verschiedenen Aufgaben der ToM-Skala und andererseits die Lektüre literarischer Erzähltexte) zumindest zum Teil auf denselben grundlegenden kognitiven Mechanismen beruhen. Die Begründung dafür lautet, dass die kognitiven Leistungsanforderungen während der literarischen Rezeption, die durch typische Merkmale von literarischen Erzähltexten entstehen, mit denjenigen der ToM-relevanten Kognitionsanforderungen innerhalb von sozialen Situationen strukturell miteinander vergleichbar sind.

Diese Überlegungen beinhalten auch methodologische Implikationen für weiterführende kognitivistisch orientierte rezeptionswissenschaftliche Arbeiten. Wenn die vorangehenden Überlegungen richtig sind, dann sollte es möglich sein, einzelne Texte daraufhin zu prüfen, welche kognitiven Anforderungen sie während der Lektüre aufgrund ihrer semantischen und strukturellen Eigenschaften an ihre Leser stellen. Die grundlegende Überlegung, die ich diesem Ansatz voranstelle lautet: Wenn sich das Sprachsystem eines Textes als strukturierte Informationsmenge verstehen lässt, die durch Leser syntaktisch und semantisch ausgewertet und zu einem kognitiven Modell der geschilderten Situation zusammengesetzt werden, dann lassen sich Texte im Umkehrschluss als auf bestimmte Art und Weise geordnetes Sets von Instruktionen zur Konstruktion eines solchen Modells analysieren. Ist dies der Fall, dann wäre es möglich, an Hand einer systematischen Analyse von Struktur und Semantik eines einzelnen Werkes, Aussagen über die kognitiven Anforderungen der Lektüre dieses Werkes zu treffen. So lassen sich die Besonderheiten einzelner Werke identifizieren, die die Lektüre jeweils auszeichnen und den Leser vor ganz bestimmte, beschreibbare Herausforderungen stellen. Das Ziel einer solchen Analyse wird es sein, diejenigen Merkmale zu identifizieren, die Einfluss auf die kognitiven Prozesse nehmen, die während der Lektüre ablaufen. Außerdem: Wenn sich die Informationsordnung eines Textes mittels narratologischer Begriffe analysieren und beschreiben lässt und wenn sich auf der anderen Seite kognitive Verstehensprozesse mit den zuvor beschriebenen Prinzipien der menschlichen kognitiven Informationsverarbeitung und der ToM charakterisieren lassen, dann sollte es möglich sein, ModellLesern an Hand einer Textanalyse bestimmte Verstehensprozesse zuzuschreiben. Der Fokus liegt entsprechend sowohl auf den typischen als auch auf den besonderen Merkmalen kon- 
kreter literarischer Werke. Inwiefern sich dies tatsächlich umsetzen lässt, möchte ich im folgenden dritten Hauptteil dieser Arbeit an Hand von zwei ausgewählten Textbeispielen genauer überprüfen. 


\section{Die Parameter der kognitiven Rezeption im Fall einzelner Werke}

\section{$1 \quad$ Zielsetzung und Fragestellung}

In den vorangehenden Abschnitten wurde deutlich, dass diejenigen kognitiven Ressourcen, die es dem Menschen ermöglichen, fremde und eigene mentale Haltungen zu verstehen und zu attribuieren, für die Rezeption literarischer Erzähltexte eine zentrale Rolle übernehmen. Die kognitiven Mechanismen, die es dem Menschen erlauben, ,eine ToM zu haben', können dabei aufgrund vergleichbarer Informationsverarbeitungsvoraussetzungen zum Teil analog zur Interpretation realer sozialer Interaktionen im Zuge der literarischen Rezeption eingesetzt werden. Darüber hinaus können gegebene kognitive Ressourcen, die in realen sozialen Kontexten eine bestimmte Funktion erfüllen, im Sinne der ,neural-exploitation'-Hypothese für literaturspezifische Verstehensaufgaben genutzt werden. In den vorangehenden $\mathrm{Ab}$ schnitten galt die Aufmerksamkeit den typischen und grundlegenden Merkmalen literarischer Erzähltexte - also vor allem ihren narrativen Merkmalen, ihrer Fiktionalität und Literarizität. Allerdings zeichnen sich literarische Texte im Allgemeinen durch eine hohe individuelle Merkmalsdiversität aus. Die hier durch die individuelle Gestaltung einzelner Werke entstehenden Differenzen betreffen sowohl ihre semantischen als auch ihre strukturellen Eigenschaften, so dass sich vermutlich auch die Kognitionsprozesse von Lesern im Einzelfall aufgrund der jeweiligen Beschaffenheit eines einzelnen Textes unterscheiden. Um diese Hypothese genauer zu untersuchen, richtet sich der Blick im Folgenden auf zwei konkrete literarische Textbeispiele. Die jeweiligen Textanalysen folgen dabei zwei verschiedenen Strategien.

Erstens: Es gilt einerseits herauszufinden, ob sich genauere Aussagen darüber treffen lassen, inwiefern die individuelle Beschaffenheit der einzelnen Texte die Kognitionsprozesse eines 
Modell-Lesers beeinflusst. Die Aufmerksamkeit richtet sich dabei auf die besondere Ausführung und Gestaltung intertextuell vergleichbarer Merkmalskategorien (wie z. B. die Beschaffenheit ihrer Erzählsituationen etc.). Ich gehe davon aus, dass in diesem Fall ein Vergleich von unterschiedlichen Texten untereinander möglich ist. Ich werde prüfen, ob sich mit einem Blick auf die Informationsstruktur beider Texte Aussagen über Abhängigkeitsverhältnisse zwischen einzelnen Textmerkmalen und der (ToM-relevanten) Kognition von Lesern treffen lassen. Ist dies möglich, dann ließe sich klären, ob die individuelle Beschaffenheit der beiden Werke unterschiedliche Informationsverarbeitungsprozesse erfordert oder evtl. die gleichen kognitiven Vorgänge in unterschiedlichem Maße gefordert sind. Um sowohl semantische als auch strukturelle Texteigenschaften systematisch und übersichtlich erfassen zu können, erfolgt die Analyse der beiden Erzähltexte hauptsächlich mit den Mitteln der Narratologie. Es sei damit jedoch nicht behauptet, eine narratologische Analyse sei die einzige Methode, um das Verhältnis zwischen Textkonfiguration und kognitiver Rezeption kenntlich zu machen. Allerdings steht mit ihr eine geeignete Vorgehensweise zur Verfügung, um bestimmte Merkmale der Informationsvermittlung zu beschreiben und auf diesem Weg die Wirkungsmechanismen zwischen Leserkognition und Textmerkmal aufzudecken.

Zweitens: Auf der anderen Seite ist zu klären, ob in den jeweiligen Texten einzelne strukturelle oder semantische Besonderheiten zu finden sind, die Leser zu einer bestimmten Rezeptionshaltung oder bestimmten Interpretationsurteilen anhalten. In diesem Fall ist ein Vergleich der zwei Werke untereinander schwierig. Daher liegt das Augenmerk an dieser Stelle nicht auf dem Vergleich der beiden Werke. Es geht lediglich darum, herauszufinden, ob bestimmte auffällige Besonderheiten des einzelnen Textes die Kognitionsleistungen des Lesers in definierbarer Weise lenken.

Ich möchte auf die im ersten Teil dieser Arbeit zusammengetragenen Kenntnisse über die sozialen Funktionen und die kognitive Funktionsweise der ToM aufbauen. Dabei gehe ich davon aus, dass sich mit dem Wissen über bestimmte kognitive Informationsverarbeitungsprozesse und einer Analyse der wichtigsten Charakteristika einzelner Texte konkrete Vorhersagen über die kognitive literarische Rezeption treffen lassen. Dabei argumentiere ich erneut basierend auf der Annahme eines Modell-Lesers, d. h. der Anspruch der folgenden Ausführungen gilt nicht der Erfassung der tatsächlich im Einzelfall ablaufenden kognitiven Prozesse eines individuellen Lesers während der Lektüre eines bestimmten Textes. Die Merkmale, die in den folgenden Textanalysen im Fokus stehen, orientieren sich an den im 
zweiten Teil dieser Arbeit bereits behandelten Merkmalskategorien. Dabei ist es wahrscheinlich, dass im jeweiligen Fall unterschiedliche Merkmalsgruppen auf unterschiedliche Weise und in unterschiedlichem Ausmaß für die literarische Rezeption relevant sind. Ein kurzer Überblick über bereits vorliegende literaturwissenschaftliche Arbeiten zu den einzelnen Werken hilft dabei, die prominentesten Eigenschaften des jeweiligen Werkes zu identifizieren, auf die ich mich konzentrieren möchte. ${ }^{40}$

Das Ziel dieser Vorgehensweise ist es, zu bestimmen, ob unterschiedliche textuell vorgegebene Parameter im Zuge der kognitiven Bildung der kohärenten Repräsentation der jeweiligen Fiktionswelt unterschiedliche kognitive Informationsverarbeitungsprozesse erfordern. Auf der anderen Seite ist denkbar, dass in beiden Fällen zwar die gleichen kognitiven Ressourcen eine Rolle spielen, die einzelnen kognitiven Kompetenzen allerdings aufgrund der Merkmalsdifferenzen in unterschiedlichem Maße gefordert werden.

Da an dieser Stelle die individuellen Differenzen literarischer Texte untereinander im Zentrum des Interesses stehen, ist es sinnvoll, zwei Werke einander gegenüber zu stellen, die sich - auf den ersten Blick - deutlich voneinander unterscheiden. Diese Vorgehensweise ist auch durch den Anspruch gerechtfertigt, die mögliche Bandbreite der Interaktionsmechanismen zwischen den für die ToM-Bildung relevanten kognitiven Ressourcen und den jeweiligen Textmerkmalen aufzuzeigen. Die Wahl fällt deshalb auf die Njáls Saga (13. Jh.), eine der größten und wichtigsten Islandsagas, und das deutlich kürzere, postmoderne deutschsprachige Werk Gehen (1972) von Thomas Bernhard. Es gilt zu zeigen, dass nicht typische, sondern auch individuelle Merkmale literarischer Erzähltexte für die Untersuchung der besonderen Rolle der ToM-relevanten kognitiven Funktionen relevant sind und in zukünftigen Forschungsarbeiten Beachtung finden müssen. Die Textbeispiele sind - in Abgrenzung zu den im Rahmen vorheriger Studien gewählten Texten - so gewählt, dass sie mentale Attribuierungsvorgänge bzw. die Themen der Wahrnehmung und Kognition nicht, oder nicht auf den ersten Blick, darstellen.

\footnotetext{
${ }^{40}$ Um einzelne Merkmale der narrativen Informationsstruktur eines Textes beschreiben zu können, greife ich erneut auf das von Genette vorgeschlagene Begriffsinstrumentarium zurück. Dabei darf allerdings nicht aus dem Blick geraten, dass sich die Spezifika einzelner Texte möglicherweise nicht in zufriedenstellender Weise den vorgegebenen taxonomischen Begriffskategorien unterordnen lassen. An solchen Stellen ist notwendig, die individuelle Abweichung der Textkonfiguration von den angelegten Begriffskategorien zu benennen und anschließend nach möglichen Implikationen für die kognitive Rezeption zu suchen.
} 


\section{Die Njáls Saga}

\subsection{Einführung I: Die Handlung der Njáls Saga}

Die gesamte Handlung, die in diesem Text geschildert ist, lässt sich im Island des sogenannten Saga-Zeitalters verorten - einer Zeitspanne von etwa einhundert Jahren zwischen der Mitte des 10. und der Mitte des 11. Jahrhunderts. Obwohl es der Titel (frei übersetzt ,Die Sage von Njáll') suggeriert und es in anderen Saga-Texten üblich ist, handelt es sich hier nicht allein um die Biographie eines einzelnen wichtigen Mannes oder die Chronik der Ereignisse innerhalb eines bestimmten Landesteils. Das Werk behandelt vielmehr, wie Einar Ólafur Sveinsson treffend feststellt, die Verflechtung von politischen und privaten Ereignissen, die mit dem großen geographischen Gebiet des isländischen Südwestens in Verbindung stehen (Sveinsson, 1971, S. 40). Um sich der detaillierten inhaltlichen Strukturierung des Textes zu nähern, ist es zunächst hilfreich, einen Blick auf seine Makrostruktur, d.h. die Abfolge der einzelnen Kapitel und Abschnitte in ihrer chronologischen Anordnung zu werfen. Die Njáls Saga lässt sich in vier übergeordnete Abschnitte einteilen. Dabei orientiert sich der Haupthandlungsstrang an den chronologisch erzählten und kausal miteinander verbundenen Verwicklungen der drei zentralen Heldenfiguren (Gunnar, Njáll und Kári) in familienübergreifende, häufig durch Dritte provozierte Konflikte. Die Njáls Saga ist außerdem in insgesamt 159 Kapitel eingeteilt, die in vielen (hauptsächlich den übersetzten) Ausgaben, Kapitelüberschriften tragen. Die einzelnen Kapitel untergliedern sich wiederum in kürzere semantische Segmente, die in der Regel aus einer Kombination von Figurenbeschreibungen und kurzen Handlungssequenzen bestehen. Sie setzen sich zumeist aus einigen einleitenden Worten des Erzählers zu Ort und Situation des Geschehens und einem Figurendialog zusammen (Lönnroth, 1976, S. 49).

Lars Lönnroths Gliederung der semantischen Einheiten des Textes (ebd., S. 24) bietet einen geeigneten Überblick über die einzelnen Episoden:

\section{Prolog}

1. Hrútur und Unnur

2. Hallgerður und ihre ersten zwei Ehen

\section{Gunnars Saga}

1. Einleitung

a) Der juristische Streit um die Rückgabe von Unnurs Mitgift 

b) Gunnars Hochzeit mit Hallgerður

2. Der Streit zwischen Hallgerður und Bergpóra

3. Der Streit zwischen Gunnar und Otkell

4. Gunnars Streit mit Egill und Starkaður

5. Der Streit mit den beiden Pórgeirs

6. Gunnars Niederlage und Tod

7. Der Streit zwischen Práin und den Söhnen Njáls

a) Práins Tod

b) Die Biographie Höskuldur Práinssons

c) Der Tod Höskuldur Njálssons

\section{Njáls Saga}

1. Die Christianisierung Islands

2. Der Mord an Höskuldur Práinson

3. Der anschließende Versuch einer Entschädigungsvereinbarung mit Flosi

4. Der Mord an Njáll und seiner Familie

5. Gerichtsverhandlungen gegen die Verantwortlichen für den Mord an Njáll

6. Káris Rachefeldzug nach dem Mord an Njáll und den gescheiterten Verhandlungen

a) Der Streit zwischen Kári und Flosi in Island

b) Fortsetzung des Streits im Ausland

c) Versöhnung

Lönnroths Gliederung bietet einen ersten Eindruck der generellen Chronologie der im Text dargestellten Ereignisse und Figuren, die im jeweiligen Abschnitt im Fokus der Aufmerksamkeit stehen. Den ersten Teil bildet ein einführender Prolog, der die wichtigsten Familien der Region und ihre soziale Verbundenheit miteinander vorstellt. Der Prolog beginnt mit der Einführung zweier wichtiger Clanfamilien, die in unterschiedlichen Teilen des Landes angesiedelt sind. Eine dieser Familien ist diejenige von Mörður Fiddle und dessen Tochter Unnur. Das Oberhaupt der zweiten Familie ist Höskuldur Dalakollsson, dessen Tochter Hallgerður später zu den wichtigsten konflikttreibenden Figuren gehören wird. Beide Familien werden durch die geplante Hochzeit von Höskuldurs Bruder Hrútur und Unnur miteinander verbunden. Hrútur heiratet Unnur nach mehrjähriger Verlobungszeit und einem Aufenthalt im Ausland am Hofe des jungen norwegischen Königs. Nach einer Liaison mit der Königsmutter während dieser Zeit spricht diese aus Eifersucht einen Fluch der Impotenz gegenüber seiner zukünftigen Ehefrau aus. Dieser Fluch wird Unnur später dazu bringen, die Scheidung zu erwirken und zu ihrem Vater zurückzukehren. 
Im zweiten Teil des Prologs richtet sich die Aufmerksamkeit auf den Beginn der Biographie von Höskuldurs Tochter Hallgerður und ihre ersten zwei Vermählungen. In beiden Ehen erhält sie nach Streitigkeiten mit dem jeweiligen Ehemann eine Ohrfeige, woraufhin sie in beiden Fällen ihren Ziehvater beauftragt, den jeweiligen Ehemann umzubringen. Zu Beginn des zweiten Teiles der Geschichte wechselt der Fokus auf Gunnar Hámundarsson, der im Zuge der ersten einführenden Charakterisierung als heldenhafte, ikonische Figur glorifiziert wird. Er ist mit Unnur verwandt und heiratet Hallgerður nach dem gewaltsamen Tod ihres zweiten Ehemannes. Nachdem es Unnurs Vater zuvor im Zuge eines legalen Verfahrens nicht gelungen war, ihre Mitgift nach der Scheidung zurück zu gewinnen, gelingt dies Gunnar dank eines Täuschungsmanövers gegenüber Hrútur und Höskuldur. Somit ist diese Episode eng mit den Ereignissen des Prologes verknüpft. Im zweiten Teil der Njáls Saga steht der soziale Aufstieg Gunnars im Fokus. Außerdem werden wichtige Figuren, wie z. B. der Titelheld Njáll in seiner Funktion als Berater in allen wichtigen juristischen Angelegenheiten, Kolskeggur (Gunnars Bruder), die Söhne von Njáll, Práinn Sigfússon und nicht zuletzt Valgarður der Graue vorgestellt. Letzterer, der später Unnur heiraten und zusammen mit ihr einen Sohn (Mörður) zeugen wird, stellt einen wichtigen Antagonisten gegenüber den Helden der Geschichte dar. Neben der Einführung wichtiger Figuren wird die Handlung von zahlreichen Zerwürfnissen zwischen Gunnar und seinen Nachbarn und Freunden getragen, die Gunnars Frau Hallgerður heraufbeschwört. Nachdem Hallgerður während einer Hungersnot ihren Bediensteten beauftragt, eine benachbarte Familie zu bestehlen und anschließend deren Scheune niederzubrennen, erhält sie auch von Gunnar - ihrem dritten Ehemann - eine Ohrfeige. Obwohl Gunnar die Streitigkeiten zunächst rechtmäßig beilegen kann, führen sie letztlich zu seiner Ächtung. Nachdem er sich weigert, seine Heimat entsprechend eines rechtskräftigen Urteils zu verlassen, fällt er - erneut durch Hallgerðurs Zutun begünstigt - seinen Rächern zum Opfer. Das Ende dieses großen zweiten Abschnittes der Saga wird nicht nur durch Gunnars Tod, sondern auch die explizite ,Entlassung' wichtiger Figuren aus dem Fokus der Handlung markiert.

Die darauffolgenden Kapitel stellen eine Brücke zwischen dem Ende der ,Gunnars Saga und dem Beginn des nächsten großen Abschnittes dar. Sie handeln hauptsächlich von der Fehde zwischen Práinn und den Söhnen von Njáll, die mit der Tötung von Práinn endet. Zu Beginn des darauffolgenden Abschnitts, indem die Titelfigur in den Fokus der Aufmerksamkeit rückt, übernimmt Njáll die Verantwortung für Práinns Sohn Höskuldur und wird dessen Ziehvater. Die enge Freundschaft, die sich daraufhin zwischen seinen leiblichen Söhnen und 
seinem Ziehsohn entwickelt, gerät nach einer erneut durch einen Dritten (Mörður) angestifteten Intrige jedoch in einen unlösbaren Konflikt. Dieser Konflikt findet seinen Höhepunkt, als Höskuldur durch die Hände seiner Ziehbrüder ums Leben kommt. Dieser Mord zieht einen Rachefeldzug nach sich, der in der Brandstiftung seinen Höhepunkt findet, wobei Njáll, seine Frau Bergpóra und ein großer Teil der Familie ums Leben kommen. Allein Njálls Schwiegersohn Kári gelingt die Flucht aus dem brennenden Haus.

Der letzte und vierte Teil des Werkes handelt von Káris Rachefeldzug gegen die Brandstifter, denen er bis nach Irland und in die Schlacht von Clontarf gegen den irischen König Brjánn folgt. Auf seine anschließende Pilgerfahrt nach Rom folgt die Versöhnung mit Flosi, dem Anführer der Brandstifter. Kári heiratet anschließend dessen Nichte Hildigunnur, die Witwe des getöteten Höskuldur. Das Werk endet mit dem Bericht von Flosis Tod und einer Liste von Káris Nachkommen, die der Ehe mit Hildigunnur entstammen. Die Versöhnung aller im Verlauf des Werkes entfachten Familienfehden ist damit vollständig.

\subsection{Einführung II: Kontext und wichtige Merkmale der Njáls Saga}

Im Jahr 870 beginnt die Besiedelung von Island durch norwegische Abtrünnige, die sich auf diese Weise der Herrschaft des norwegischen Königs Haraldr Hárfagri (Harald ,Schönhaar`) entzogen. Der Prozess der Landnahme dauerte nur wenige Jahre und war im Jahr 930 weitestgehend abgeschlossen (Edwards \& Pálsson, 2006, S. 1 f.). Die folgenden Jahre zwischen 930 und 1030 gelten als ,Sagazeitalter' (söguöld), da sich die meisten in der Saga Literatur beschriebenen Ereignisse in dieser Periode abspielen. Es folgt bis in das Jahr 1120 hinein das Zeitalter des Friedens (friðä̈ld) und anschließend die Epoche des Schreibens, das sogenannte ritöld, in der die klassischen Saga Texte, deren Inhalte bis dahin mündlich tradiert worden waren, zum ersten Mal niedergeschrieben wurden. Im Jahr 1262 endet die Unabhängigkeit Islands, das von nun an unter die Krone Norwegens steht. ${ }^{41}$ Obwohl die meisten klassischen Sagatexte von den Ereignissen des söguöld berichten, stammen die ältesten Manuskripte aus dem mittleren bis späten 13. Jahrhundert. Es liegen also mehr als 200 Jahre zwischen den Ereignissen, von denen die Sagas berichten und den Autoren, die diese zusammen mit detaillierten Genealogien in schriftlicher Form festhielten. Die Njáls Saga erzählt

\footnotetext{
${ }^{41}$ Eine übersichtliche geschichtliche Zusammenfassung der Ereignisse ist bei Liestøl (1930) zu finden.
} 
die Geschichte der Familien und Clans, die im 11. Jahrhundert den Süden Islands besiedelten. Die einzelnen Schicksale dieser Familien sind durch Fehden und Hochzeiten miteinander verbunden. Das Werk findet seinen tragischen Höhepunkt in der Verbrennung des angesehenen Rechtsgelehrten Njáll ${ }^{42}$ und seiner Familie in dessen eigenem Haus. Ein Abgleich mit zahlreichen historischen Quellen lässt vermuten, dass in der Njáls Saga nicht nur fiktive, sondern auch eine Reihe historischer Ereignisse geschildert werden. In einigen Quellentexten sind unter dem Stichwort ,Njálsbrenna' beispielsweise Verweise auf den im Werk geschilderten zentralen Vorfall des Morbrandes zu finden, dem Njáll und seine Familie zum Opfer fallen (Sveinsson \& Schach, 1991, S. 7). Diese Tatsache, zusammen mit einigen textinhärenten Merkmalen, wie bspw. dem Sprachduktus und den detaillierten Familiengenealogien, begründen den unter den Forschern der Skandinavistik selten angefochtenen Konsens, dass die erzählten Ereignisse zumindest teilweise auf historischen Begebenheiten beruhen (Ebd.). Beispiele für die Vermittlung historischer Informationen sind die Passagen, die der Einführung des Christentums in Island und der Schlacht von Clontarf gewidmet sind. Beide Ereignisse sind nicht nur in der Njáls Saga beschrieben, sondern sind darüber hinaus in zahlreichen weiteren Quellen dokumentiert (Fox, 1963, S. 290). Über die Figur des Njáll findet sich ein kurzer Eintrag im Landnámabók (Landnahmebuch):

With Asgerd's approval, her brother Thorolf took possession of land west of Fljot, between the two Deildar Rivers, making his home at Thorolfsfell. There he fostered Thorgeir Gollnir, son of Asgerd, who farmed there afterwards. Thorgeir's son was Njal who was burnt to death in his house. (Edwards \& Pálsson, 2006, S. 129)

Die Diskussion darüber, zu welchem Grad der Text als historisch einzuordnen ist, hat bis heute keinen Abschluss gefunden. Der isländische Student Olavus Olavius, der im Jahr 1772 die erste wissenschaftlich edierte Ausgabe des Werkes in Kopenhagen herausgab, klassifizierte die Njáls Saga in diesem Zusammenhang noch als Geschichtswerk, das vor allem dazu dienen soll, modernen Lesern Wissen über die politischen und juristischen Verhältnisse des isländischen Commonwealth-Zeitalters zugänglich zu machen (Lönnroth, 1976, S. 2). Diese extreme Ansicht wird von den heutigen Forschern nicht mehr vertreten, auch wenn noch immer ein großes wissenschaftliches Interesse an der Identifikation historischer Ereignisse und Figuren und ihrer Unterscheidung von den fiktiven Anteilen des gesamten Werkes besteht. Knut Liestøl (1930, S. 182) schreibt:

\footnotetext{
${ }^{42}$ Außerhalb von zitierten Passagen aus den Werkausgaben und der Forschungsliteratur orientiert sich die Schreibweise der Figuren-Namen an der modernen isländischen Grammatik.
} 
[...] the sagas are our most important source of information regarding the history of Iceland during a period of more than a hundred years; and the information they contain regarding the life and customs of those times is of immense value for the study, not only of Icelandic, but of Scandinavian and even of Germanic culture.

Trotz der Verbindungen zu historischen Figuren, Stoffen und Motiven wurde der Text im skandinavistischen Forschungsdiskurs auch als Kunstwerk und fiktionales Narrativ behandelt. Einer der Hauptvertreter dieser Ansicht ist Einar Ólafur Sveinsson, der basierend auf den Manuskriptfragmenten, die der skandinavistischen Mediävistik vorliegen, eine neue Gesamttextausgabe erstellte und sie im Jahr 1954 herausgab. ${ }^{43}$ Form und Inhalt aller modernen isländischen Textausgaben beruhen auf dieser Edition.

Es wird vermutet, dass das Werk zirka im Jahr 1280 entstand, obwohl sich die erzählten Ereignisse in den Jahrzehnten rund um das Jahr 1000 n. C. abspielen. Der nachweisbare intertextuelle Einfluss des Gesetzesbuches Járnsíða, welches erst im Jahr 1271 in Island eingeführt wurde, hilft dabei, die Entstehungszeit auf einen Zeitraum zwischen diesem Jahr und der Zeitpunkt der Niederschrift des ältesten überlieferten Manuskriptes (ca. 1300) eingrenzen (Sveinsson, 1971, S. 11). Neben der ausführlichen Forschung zur Editionsgeschichte stehen im Forschungskanon zur Njáls Saga einige wenige wichtige Fragen im Zentrum des Interesses. Eine wichtige Frage, die im Zuge der Forschung zu diesem speziellen Werk häufig gestellt wurde, betrifft die Identität seines Autors bzw. seiner Autoren. Obwohl die These, dass alle überlieferten Manuskriptfragmente auf ein originales Manuskript mit der Handschrift eines einzelnen Autors zurückgehen, mehrfach in Frage gestellt wurde, hielt sich diese Überzeugung im Verlauf der Forschungsgeschichte hartnäckig (ebd.). Sveinsson, der selbst diese Ansicht vertritt, beruft sich in diesem Zusammenhang auf die enge Verknüpfung historischer und fiktiver Ereignisse, die kunstvolle Komposition des Textes, die Einheitlichkeit des Erzählstiles sowie der Charakterskizzen und folgert daraus, dass es sich im Fall der Njáls Saga nur um das Werk eines einzigen, artistisch begabten Autors handeln kann (ebd.). Zur Identität dieses Autors liegen einige theoretische Überlegungen vor, von denen sich letztlich keine endgültig bestätigen ließ. ${ }^{44}$ Den entscheidenden Hinweis zur Beantwortung der Frage nach der Identität des Autors zu finden, ist bis heute nicht gelungen. Aufgrund der literatur- und kulturgeschichtlichen Entstehungszeit des Werkes ist es m. E. durchaus

\footnotetext{
${ }^{43}$ Diese Ausgabe wird an relevanten Stellen, z. B. wenn die moderne Übersetzung in relevanter Form von der originalsprachlichen Fassung abweicht, herangezogen und als ,NSI' zitiert.

${ }^{44}$ Diese Überlegungen sind z. B. bei Lönnroth (1976, S. 4) zusammengefasst.
} 
möglich, dass es sich nicht um das Werk eines einzelnen Autors, sondern um ein Konglomerat von Textstücken handelt, die als Niederschriften mündlich tradierter Geschichten zu bewerten sind..$^{45}$ Dieses Problem ist nicht Gegenstand der vorliegenden Arbeit und soll daher hier nicht ausführlich diskutiert werden. Wichtig ist allerdings, dass Leser dieses Werk nicht wie im Fall eines zur heutigen Zeit üblichen ,Autorenwerkes' einer bestimmten historischen Autorfigur zuordnen können.

Ein auffälliges Merkmal der Njáls Saga sind die inhaltlichen Exkurse über wichtige und gut dokumentierte historische Ereignisse, wie das Kapitel über die Christianisierung Islands (in den Jahren 999 - 1000) und die ausführliche Darstellung der Schlacht in Clontarf (1014) (Naumann, 2011, S. V). Diese Episoden sind als thematische Exkurse in die Chronologie der Gesamthandlung eingebunden. Sie besitzen eine eigene in sich geschlossene Mikrostruktur, und können - Lars Lönnroth zufolge - neben ihrer Rolle im Gesamtzusammenhang des Werkes auch als unabhängige Textpassagen analysiert werden (Lönnroth, 1976, S. 31). Die Einbindung der zwei thematischen Exkurse in die Haupthandlung lässt vermuten, dass es sich bei der Njáls Saga um ein Konglomerat mehrerer, bereits zuvor existierender Narrative handeln könnte. Gleichzeitig drängt sich die Frage nach dem Fiktionsstatus aller geschilderten Ereignisse auf, die in der mediävistischen Forschung häufig Anlass zu der Suche nach möglichen historischen Quellen des gesamten zusammengestellten Stoffmaterials des Werkes gab. ${ }^{46}$ Sveinsson (1971, S. 12) identifiziert einige dieser poetischen, mythischen und kulturhistorischen Quellen, aus denen sich der Stoff des Haupttextes speist und einige intertextuelle Verlinkungen, die an dieser Stelle auf Grund der Breite des Feldes nicht einzeln wiedergegeben werden können (ebd., S. 14). Die zum Teil erfolgreiche Suche nach Quellen für die dargestellten Stoffe und Motive rechtfertigt jedoch die Frage nach dem Fiktions- bzw. Geschichtsstatus einzelner Figuren und Handlungselemente, die einen Schwerpunkt innerhalb der Forschung zur Njáls Saga bildet. ${ }^{47}$ Trotz der Sonderstellung dieser zwei Abschnitte bezüglich ihrer Thematik und inhärenten Struktur sind sie auf inhaltlicher Ebene durch kausale Verknüpfungen mit der Haupthandlung verbunden, so dass der Eindruck einer zusammenhängenden Gesamtstruktur entsteht (Lönnroth, 1976, S. 23).

\footnotetext{
${ }^{45}$ Eine gute Einführung in die Bedeutung mündlicher Traditionen für die Entstehung der Njáls Saga, wie sie heute in publizierter Form vorliegt in der Arbeit von Liestøl (1930).

${ }^{46}$ Eine Zusammenfassung dieser Forschungsbemühungen und der entsprechenden Ergebnisse findet sich bei Lönnroth (1976, S. 39 ff.). Auch Andersson (1964, S. 41 ff.) bespricht dieses Problem.

${ }^{47}$ Eine Zusammenfassung der Probleme der Editionsgeschichte in dieser Hinsicht findet sich bei Andersson (1964, S. 41 ff.).
} 
Die Frage nach der ästhetischen Funktion von strukturellen und semantischen Eigenschaften und damit nach dem Kunstcharakter der Njáls Saga wurde in der Forschungsgeschichte zu diesem Werk erst spät gestellt. Erst Lars Lönnroth (1976, S. 22) sucht nach den ästhetischen Elementen des Textes, wobei zuvor überwiegend die Suche nach der Identität seines Autors, nach den historischen Elementen der Geschichte sowie seiner Editionsgeschichte im Fokus gestanden hatten. Mit einem Blick auf die Gattungsgeschichte der Saga-Literatur schreibt Lönnroth zur erzählerischen Strategie der kausalen Handlungsmotivierung in der Njáls Saga: „This is the literary tradition the author of Njála follows. More radically than any previous writer of long sagas, however, he tries to tie everything together in his ,network of events““ (ebd., S. 209). Die Njáls Saga zeichnet sich im Vergleich zu anderen Texten seiner Gattung über eine ungewöhnlich dicht verwobene innertextuelle Strukturierung aus: Sveinsson (1971, S. 54 f.) schreibt:

From beginning to end Njála is an articulated, unified complex of events, all of which precipitate others. The initial impulse is not a single event, but many individual and originally unrelated events [...]. Thus one event springs from the other, and the reader often has the feeling that these events are tied together, one with the other.

Die Njáls Saga gilt als das umfangreichste und, so der Konsens der skandinavistischen Literaturwissenschaft, als das künstlerisch bedeutsamste Beispiel der klassischen isländischen Saga Literatur (Naumann, 2005, S. III). Sie ist ein wohlstrukturiertes Narrativ, das möglicherweise bereits existierende Stoffe aufgreift, allerdings von einem unbekannten Künstler in seiner ästhetischen Form zusammengestellt und strukturiert wurde (Lönnroth, 1976, S. 2). Die moderne Skandinavistik kategorisiert diesen Text dementsprechend als komplexes Kunstwerk und nicht als historisches Dokument (ebd., S. 3).

Mit Blick auf die wichtigsten Fragestellungen der Forschungsgeschichte zur Njáls Saga möchte ich im Folgenden zunächst den möglichen Einfluss wichtiger allgemeiner Faktoren auf die kognitive Rezeption des Textes beleuchten. Solche Faktoren sind z. B. das fehlende Wissen um die Autoridentität sowie der Bezug der dargestellten Ereignisse zur Realität. Die Frage lautet hier, ob und an welchen Stellen solche Faktoren die online-Rezeption des Werkes beeinflussen lassen. Ich möchte klären, ob sich im Zuge der Textanalyse selbst mit Blick auf diese Faktoren begründete Annahmen formulieren lassen. Auf der anderen Seite möchte ich im Rahmen der Textanalyse die in der Forschung erwähnte Frage nach besonderen strukturellen Strategien der Verknüpfung einzelner Handlungsepisoden, der als Leitfaden meiner Analyse nutzen. Es gilt hier zu klären inwiefern durch die Beschaffenheit des Textes selbst 
der Eindruck der strukturellen Motivierung der Gesamthandlung entstehen kann, der Forscher in der Vergangenheit zu dem Schluss führte, dass Werk sei ein dicht motiviertes und strukturiertes Narrativ. Darüber hinaus möchte ich mit Blick auf den komparatistischen Ansatz dieses Kapitels eine Grundlage für einen Vergleich schaffen und die Besonderheiten der Erzählsituation im Zusammenhang mit der kognitiven Rezeption des Werkes genauer untersuchen. ${ }^{48}$

\subsection{Die textgelenkte Rezeptionshaltung des Lesers}

\subsubsection{Fiktivität vs. Historizität}

Wenn ein Leser die Njáls Saga zur Hand nimmt und zu lesen beginnt, wird die Lektüre von Fragen begleitet, die zum Teil die noch immer offenen Fragen der isländischen Literaturgeschichtsforschung wiederspiegeln. Eine erste Frage betrifft die Identität des Autors. Ist überhaupt sinnvollerweise von einem Autorenwerk auszugehen oder handelt es sich vielmehr um die Niederschrift von zuvor mündlich tradierten Stoffen? Die zweite Frage lautet: Sind die dargestellten Ereignisse historisch oder fiktiv? Der Text, der modernen Lesern z. B. in Form eines gewöhnlichen gebundenen Buches vorliegt, lässt sich keinem konkreten historischen Autor zuschreiben. Auch wenn ich an dieser Stelle die Frage nach der Identität des Autors nicht lösen kann und möchte, ist dieses Problem für die in dieser Arbeit gestellten Fragen relevant. Entscheidend für die kognitive Rezeption des Werkes ist, dass - wenn einem Werk keine historische Autorfigur zugeordnet werden kann - die ,mentale‘ Kommunikationsstruktur, die im Fall eines ,Autorenwerkes‘ zwischen Autor und Leser durch die Attribution z.B. von Kommunikations- und Aussageintentionen entsteht und die ich im zweiten Hauptteil dieser Arbeit näher beschrieben habe, beeinflusst wird. Es stellt sich die Frage, ob und

\footnotetext{
${ }^{48}$ Es stellt sich - mit Blick auf die nachfolgenden Untersuchungen und auf die lange Tradierungs- und Editionsgeschichte des Textes - die Frage nach der Wahl einer günstigen Werkausgabe, die diesen Analysen zugrunde liegen soll. Daher wird der vorliegenden Untersuchung die modernisierte und vielfach übersetzte Fassung der Njáls Saga zugrunde gelegt. Da auch die verschiedenen modernen Ausgaben zum Teil erheblich voneinander abweichen und um eine Sprachbarriere zu vermeiden, beziehe ich mich überwiegend auf eine ausgewählte moderne englischsprachige Ausgabe (nachfolgend zitiert als ,NSE'), die heutigen Lesern zur Verfügung steht, wenn sie sich dafür entscheiden, die Njáls Saga lesen zu wollen. Inhalt und Formulierungen habe ich im Vorfeld sowohl mit anderen modernen englischsprachigen als auch mit einer deutschsprachigen Ausgabe und der kritischen Ausgabe Sveinssons (1954) in Altisländischer Sprache (nachfolgend zitiert als ,NSI') verglichen. Aufgrund des begrenzten Rahmens dieser Dissertation und um die zentrale Fragestellung nicht aus dem Blick zu verlieren werde ich lediglich auf Varianzen zwischen den einzelnen Ausgaben hinweisen, die m. E. für diese Arbeit relevant sind.
} 
inwiefern Lesser Aussage- und Fiktionsintentionen attribuieren, wenn kein konkreter Autorbekannt ist, dem entsprechende mentale Haltungen zugeschrieben werden können. Es ist zu vermuten, dass ein Modell-Leser, der die Kulturgeschichte Islands und vielleicht sogar die Merkmale der Saga Texte kennt, dieses Wissen bei der Rezeption des Textes berücksichtigt. Er geht womöglich davon aus, dass in der Njáls Saga zumindest zum Teil historische Ereignisse tradiert werden. Es ist sogar möglich, dass es zu seinen Rezeptionszielen gehört, die historischen Ereignisse und Figuren zu identifizieren, die ihm dank seines Wissensschatzes bekannt sind. Ich habe bereits an einleitender Stelle begründet, dass sich eine solche Fragestellung zu den Rezeptionszielen von Lesern im Rahmen theoretischer Arbeiten höchstens spekulativ beantworten lässt. Es wären eine Reihe von Variablen zu berücksichtigen, die sich im Rahmen der vorliegenden (textorientierten) Analyse nicht erfassen lassen. Daher möchte ich an dieser Stelle das Augenmerk zurück auf die Gruppe textzentrierter Hypothesen lenken. Dabei lautet die Frage erneut: Lässt sich auch durch einen Blick auf die konkreten Merkmale des Textes ableiten, inwiefern die Rezeptionshaltung eines Lesers beeinflusst werden könnte? Angenommen ein moderner Leser ohne Vorbildung über die Kulturgeschichte Islands und die Gattung der Saga Texte nimmt (heute) die Njáls Saga in ihrer übersetzten und gebundenen Buchform zur Hand und beginnt zu lesen. ${ }^{49}$ Auf Basis welcher Informationen entscheidet er, wie er die geschilderten Ereignisse verstehen und in welchen Bezug er sie zur historischen Realität setzen soll? Ich möchte mit einem genaueren Blick in den Text hinein untersuchen, ob besondere fiktionsanzeigende (oder entsprechenden historizitätsanzeigende) Signale zu finden sind, die die Rezeptionsprozesse des Lesers auf vorhersehbare Weise lenken können. Hier geht soll es nicht darum gehen, Hypothesen über grundsätzliche Rezeptionshaltungen eines Lesers gegenüber dem Text zu bilden, sondern Aussagen über konkrete Merkmale des Textes zu machen und zu prüfen, ob sie Leser evtl. zu einer bestimmten Rezeptionshaltung anhalten. Dabei ist nicht gesagt, dass dies im empirischen Einzelfall tatsächlich eintritt. Dennoch gehe ich davon aus, dass bestimmte Merkmale des Textes die Informationsverarbeitung seitens des Lesers beeinflussen und auf diesem Weg bestimmte Rezeptionshaltungen begünstigen. Diese lassen sich mit einem Blick auf Struktur und Inhalt des Textes bestimmen. Die Informationsverarbeitung hängt zum einen von der kognitiven Disposition des Lesers und zum anderen von der Beschaffenheit der

\footnotetext{
${ }^{49}$ Es ist denkbar, dass an dieser Stelle der kulturelle Rezeptionskontext eine wichtige Rolle spielt. Sehr allgemein gesprochen gehe ich gehe davon aus, dass Leser zu einer anderen Zeit und in einem anderen kulturellen Raum Literatur anders rezipieren. In dieser Arbeit fehlen die Mittel und der Raum, um diese Möglichkeit genauer zu untersuchen. Dennoch darf nicht außer Acht gelassen werden, dass diese Variablen bei der Beantwortung der gestellten Frage eine Rolle spielen.
} 
Informationsstruktur im Text ab. Sie lässt sich im Zuge einer Textanalyse bestimmen. Damit ist eine Grundlage für begründete Schlüsse über die kognitiven Interpretationsprozesse seitens des Lesers geschaffen. Wie bereits angedeutet gehe bei den folgenden Überlegungen von einem modernen Leser aus, der sich weder mit der Geographie Islands, noch mit den historischen Quellen über Ereignisse und Figuren, die in diesem Werk dargestellt werden, im Detail auskennt. Es geht vor allem darum zu klären, ob ein solcher ,naiver‘ Leser, der über keine tiefergehenden literaturhistorischen Kenntnisse verfügt, allein aufgrund der spezifischen Textmerkmale zu einer bestimmten Rezeptionshaltung geführt wird. Es soll hingegen an keiner Stelle darum gehen, zu klären, ob die geschilderten Ereignisse und Figuren tatsächlich als fiktiv oder historisch zu bewerten sind.

In der Njáls Saga finden sich eine Reihe von Passagen, in denen Ereignisse geschildert werden, die sich mit unserem heutigen Realitätsverständnis nicht vereinbaren lassen. Es werden z. B. Szenen geschildert, in denen heidnische Magie, christliche Wunder oder Geistererscheinungen eine Rolle spielen. Wenn Textpassagen Elemente enthalten, „[...] die von dem im Hinblick auf die gültige Wirklichkeitskonzeption Möglichen abweichen“ (Zipfel, 2001, S. 109), dann sind sie als Fiktionssignale zu bewerten, die der Ebene der Geschichte zuzurechnen sind. Zipfel (ebd., S. 234) schreibt auch: „Wenn in der Geschichte einer Erzählung sich Dinge ereignen, die nach den Vorstellungen der herrschenden Wirklichkeitskonzeption als nicht möglich angesehen werden müssen, wird der Leser die erzählte Geschichte als fiktiv ansehen und damit den Erzähl-Text als fiktionalen rezipieren.“ Bei einem Blick in den Text der Njáls Saga hinein, sind eine Reihe solcher Elemente, die Zipfel als Fiktionssignale klassifizieren würde, leicht zu identifizieren:

$\mathrm{Zu}$ einem recht frühen Zeitpunkt der Lektüre wird eine Figur (Svanur) eingeführt, die als ,great wizard' (NSE, S. 23) bezeichnet wird. Mit Hilfe heidnischer Magie bewirkt Svanur, dass Ósvífur und seine Gefolgsleute an ihrem Rachfeldzug gegen den Ziehvater seiner Nichte gehindert werden:

Svan took a goatskin and wrapped it around his head and spoke: ,Let there be mist and mischief, and let marvels eke befall all those who as foes seek you!" Now to tell how Ósvíf and his men rode along the mountain ridge. They were met by a thick fog and Ósvíf said: ,This is Svan's doing, and we shall be lucky if no worse follows.

A little while later great darkness fell on their eyes, so that they could see nothing. They dropped from their saddles and lost their horses; some fell into bogs and some lost their way in the woods so as to come to harm, and they lost their weapons too. 
Then Ósvíf said: ,If I could only find my horses and weapons again, I would turn back.

As soon as he had said this, they were again able to see somewhat and they found their horses and weapons. Then many of the men urged that they continue with their pursuit. They did so and the same foul play happened again. This went on three times. (NSE, S. 27 f.)

An einer anderen Stelle, nach dem Tod von Gunnar, öffnet sich dessen Grab und er erscheint als Geist, der einige Verse zitiert:

Once it happened that Skarphedin and Hogni were standing outside and to the south of Gunnar's Cairn. There was bright moonlight, but now and then it was dimmed by a passing cloud. Then it seemed them as though the cairn were standing open; Gunnar had turned around in his grave and was looking at the moon. They thought they saw four lights burning in the cairn; yet none of them seemed to cast any shadow. They saw that Gunnar was happy and wore a cheerful expression. He recited this verse, and so loudly that Skarphedin and Hogni could have heard it clearly even if they had been standing farther away. (NSE, S. 153)

Neben dem heidnischen Zauber und der Geistererscheinung sind an anderen Stellen christliche Wunder beschrieben. So besiegt der Missionar Pangbrandur einen Berserker mittels eines wundersamen Feuers und einer Berührung mit dem Kruzifix (NSE, S. 209). Wenig später geschieht auch zugunsten des rachesuchenden blinden Ámundi ein Wunder Gottes, nachdem dessen Vater gewaltsam zu Tode kam und der Verantwortliche (Lýtingur) sich weigert, eine Kompensation zu zahlen:

,I cannot understand how that can be just before God, for you have hewn so close to me,' answered Ámundi. ,However, I can tell you this: if I were blessed with sight in both my eyes, I would demand full redress for my father's death or else seek revenge! Yet I shall let God decide between us.

[...] Suddenly his eyes opened and he said: ,Praised be the Lord! I can now see what his will is!

With that he [...] went up to Lýting, and drove his axe into his head right up to the hammer of the axe and then pulled it out. Lýting fell forward and was dead immediately. Ámundi returned to the door of the booth, and as he came to the same place where his eyes had opened, they now closed again, and he remained blind the rest of his life. (NSE, S. 213)

Ein entsprechendes Fazit über die Njáls Saga könnte an dieser Stelle lauten: Ein moderner Leser wird diesen Text aufgrund der geschilderten Ereignisse, die nicht mit der heutigen Wirklichkeitskonzeption vereinbar sind, als fiktionalen Text rezipieren. Belegen ließe sich 
diese Hypothese nur auf empirischem Weg. Mit Blick auf den Forschungsansatz der vorliegenden Arbeit stellt sich jedoch noch eine andere Frage: Vermittelt der Text seinem Leser eventuell auch Hinweise über die Kommunikations- und Fiktionsintention der im Text zusammengestellten Aussagen? Ist dies möglicherweise sogar unabhängig davon möglich, dass ein bestimmter Autor als ,Urheber` der Aussagen angenommen werden kann? Welche Entscheidung trifft ein Leser mit den zuvor genannten Eigenschaften allein anhand der ihm vermittelten Informationen darüber, ob in der Saga historische oder fiktive Ereignisse vermittelt werden sollen? Es fällt auf, dass sich im Text selbst im Gegenzug zu den genannten fiktionsanzeigenden Passagen auch eine Reihe von Abschnitten, Formulierungen und Merkmale finden lassen, die ich in Anlehnung an Zipfels Modell der Fiktionssignale ,Historizitäts'-Signale nennen möchte. Auf den ersten Blick fallen in diesem Zusammenhang die detaillierten Genealogien auf, die mit der Einführung der wichtigsten handelnden Figuren in die Geschichte einhergehen. Sie sind - in ihrer Ausführlichkeit - für die Motivierung der Geschichte nicht notwendig, vermitteln aber den Eindruck der historischen Verankerung der Figuren in den Stammbäumen der isländischen Familien, die das Land im Mittelalter besiedelten. Auch wenn einem Leser das Wissen über den geschichtlichen Hintergrund der Besiedelung fehlt, wird durch die ausführliche und zu großen Teilen unmotivierte Darstellung der Familienabstammung der Charakter einer Chronik unterstrichen. Dies wird außerdem durch die Wortwahl des Saga-Erzählers an vielen Stellen unterstützt. Duktus und Wortwahl des Erzählers suggerieren, dass er sich als Erzähler eines bereits vorhandenen Stoffes (Im Gegensatz zu einem Erzähler einer fiktiven Geschichte) versteht. Er macht an vielen Stellen sowohl implizit als auch explizit deutlich, dass die berichteten Geschehnisse einem bestehenden Stoff entnommen und lediglich erneut wiedergegeben werden. Besonders augenscheinliche Beispiele für solche Passagen sind jene, in denen die Wissenslücken der Erzählinstanz bezüglich der Geschehnisse, die sich ,tatsächlich‘ abgespielt haben, sichtbar werden. Im Zuge der ausführlichen Beschreibung einer Festgesellschaft steht zum Beispiel folgende Bemerkung des Erzählers, die einen solchen Moment illustriert: „We are not told how the other seats were assigned“" (NSE, S. 65). ${ }^{50}$ Da der Erzähler hier lediglich als Sprachrohr für einen zu überliefernden Stoff auftritt, ist es ihm nicht möglich, die Informationen, die ihm über die Ereignisse nicht bekannt sind, an den Leser weiterzugeben. Auf diesem Weg verstärkt sich der Eindruck, dass der Leser es nicht mit dem Urheber einer ,erfundenen`

\footnotetext{
${ }^{50}$ Alle Hervorhebungen dieser Art hier und in den im Folgenden zitierten Textpassagen sind meine Hervorhebungen.
} 
Geschichte, sondern eher mit einem Berichterstatter zu tun hat, der über historische Ereignisse berichtet, über die ihm - dem Berichterstatter - eine Reihe von Informationen fehlen. Die Figuren und Ereignisse, von denen die Rede ist, sind demnach Teil einer überlieferten und durch die Erzählung lediglich erneut evozierten Geschichte.

Der Erzähler unterstreicht an anderen Stellen, dass er nicht der eigentliche ,Ursprung‘ der Geschichte ist, sondern wiedergibt, was bereits mündlich oder schriftlich tradiert wurde. Über die Figur Thjóstólfs wird zum Beispiel an entsprechender Stelle gesagt: „He was a strong man and well skilled in arms. He had slain many men and had never paid any reparations for any one of them. It was said that he was hardly the type of man to improve Hallgerd's character" (NSE, S. 21). Die Wortwahl in dieser Passage suggeriert, dass nicht der Erzähler selbst das Urteil über die Figur fällt. Es heißt hier nicht: ,He was hardly the type of man to improve Hallgerd's character. 'Vielmehr scheint das Urteil bereits an anderer Stelle überliefert worden zu sein, (,It was said that... ) und der Erzähler gibt es an dieser Stelle in einer Form wieder, die eine Überlieferung der Information impliziert. Es ist meine Vermutung, dass auch ein ,naiver' moderner Leser, der über kein Wissen über diese Erzähltraditionen verfügt durch die auffälligen Erzählstrategien innerhalb des Textes in seiner Rezeptionshaltung beeinflusst wird. Ihm begegnen im Verlauf der Lektüre eine Reihe von (nach Frank Zipfels Modell) als geschichtsinterne Fiktionssignale zu klassifizierende Handlungselemente, sowie Darstellungsformen und Erzählelemente, die das Werk als erzählerische Wiedergabe eines (evtl. historischen) Stoffes auszuzeichnen scheinen. Hinzu kommt, dass einem Leser als ,mentaler' Kommunikationspartner für die Attribution von Aussageoder Fiktionsintentionen keine historische Autorpersönlichkeit bekannt ist. Dafür sieht er sich mit einem heterodiegetischen Erzähler konfrontiert, der die erzählten Ereignisse und Figuren spürbar als Teil eines überlieferten Stoffes kennzeichnet. Hinzu kommt der nüchtern-objektive und handlungs- bzw. ereignisorientierte Erzählstil, den der Erzähler wählt. Hauksson und Óskarssin schreiben, dass sich dieser Stil im Allgemeinen durch kurze, schlicht strukturierte Sätze auszeichnet, in denen sich kaum gelehrte (z. B. lateinische) Einschläge finden lassen. Auf rhetorischen Schmuck wird im Gegensatz zu anderen literarischen Gattungen des isländischen Mittelalters beinahe vollständig verzichtet (Hauksson \& Óskarsson, 1994, S. 274 f.).

Ich gehe davon aus, dass die Verstehensprozesse eines (Modell-) Lesers u. a. durch die Beschaffenheit des Textes gelenkt und sich daher zu gewissen Teilen daran ablesen lassen. Ich gehe weiterhin davon aus, dass sich die Rezeptionshaltung eines Lesers an einem ,mentalen“ 
Kommunikationspartner orientiert, dem bestimmte Aussage- und Fiktionsintentionen attribuiert werden können. Auf dieser Basis und mit einem Blick auf die Situation eines Lesers, der sich der Lektüre der Njáls Saga zuwendet, lautet ein möglicher Schluss, dass die Rolle des ,mentalen“ Kommunikationspartners hier durch die durch den Text evozierten Erzählinstanz ausgefüllt werden kann, der sich als Erzähler eines bestimmten Stoffes ausweist, aus dem er Elemente bewusst auswählt, in bestimmter Form zusammenstellt und an den Leser vermittelt. Um die Bedeutung und den Einfluss der gewählten Erzählstrategien genauer zu erfassen, möchte ich die Konstitution der Erzählsituation im kommenden Abschnitt eingehender beschreiben und prüfen, welche Bedeutung sie für die kognitiven Interpretationsprozesse seitens des Lesers hat.

\subsubsection{Parameter für die kognitive Interpretation - die Erzählinstanz der Njáls Saga}

In einer realen sozialen Interaktion nutzen wir unser Vor- und Kontextwissen sowie verschiedene Sinneseindrücke und Kommunikationspartner als Informationsquellen, um bestimmte Situationen interpretieren zu können. Bei unserem Verstehen einer literarisch dargestellten Welt, sind wir auf die Angaben der Erzählinstanz angewiesen. Informationen über eine erzählte Welt sind in diesem Sinne immer im eigentlichen Sinn des Wortes, vermittelt'. Genette schreibt: „Ohne narrativen Akt gibt es folglich keine narrative Aussage und mitunter nicht einmal einen narrativen Inhalt" (Genette, 2010, S. 11). Ein narrativer Akt hat einen Urheber, der im narratologischen Diskurs wie auch in dieser Arbeit mangels eines besseren Begriffes ,Erzähler` oder ,Erzählinstanz‘ genannt wird. Es ist hier kein Raum dafür, die Problematik der Kategorisierungsbemühungen zu diskutieren. Auch eine genauere Aufschlüsselung verschiedener Ansätze zur Typologisierung diegetischer Strukturen ist hier nicht zielführend. Relevant ist an dieser Stelle allerdings, dass der Erzählakt als Akt der strukturierten Informationsvermittlung zu beschreiben ist, durch den ein Leser eine Reihe von Informationen in einer bestimmten Form und Reihenfolge vermittelt bekommt, auf deren Basis kognitive Interpretationsprozesse ablaufen. Die Konfiguration der Informationsvermittlung bestimmt, welche Art und welche Menge Informationen einem Leser zur Verfügung stehen und damit welche kognitiven Interpretationsschritte er selbst gehen muss, um zu einem kohärenten Textverständnis zu gelangen. Durch eine Analyse der Textstrukturen so der Ansatz dieser Analyse, lässt sich erschließen, welche kognitiven Leistungen ein Leser während der Lektüre einer bestimmten leisten kann und muss. Ich gehe davon aus, dass sich unter Berücksichtigung bestimmter Annahmen (im Sinne der Bestimmung eines Modell- 
Leser-Konstruktes) und mit Hilfe einer genauen Analyse der Informationsstruktur eines Textes Schlüsse auf die Kognitionsbedingungen seitens eines Lesers ziehen lassen. Damit lässt sich das ,Profil‘ kognitiver Leistungsanforderungen eines Textes erstellen. Im Folgenden ruht die Aufmerksamkeit auf den Informationsstrukturen der Njáls Saga und den kognitiven Aufgaben die sich infolgedessen ihrem Leser stellen.

Wie also ist die Erzählinstanz der Njáls Saga zu charakterisieren? Die Konfiguration der Erzählinstanz der Njáls Saga entspricht - auf den ersten Blick - den genretypischen Gestaltungsmerkmalen der Saga-Literatur. Allen schreibt über einen solchen Erzähler:

The narrator [...] reports only those facts, deeds, and speeches essential to the forward motion of the story. The saga-man moreover restricts his viewpoint. $\mathrm{He}$ is not an omniscient narrator. Scenes are presented as they might be viewed by an alert observer close to the participants. What these participants say and do may be heard and seen, but what they think and what moves them to act is left for the audience to decide. (Allen, 1971, S. 99)

Der Erzähler der Njáls Saga bildet hier keine Ausnahme. Er steht außerhalb des Geschehens und verfügt über einen umfassenden Überblick über die Ereignisse und Figuren, über die er berichtet. Er beansprucht außerdem Autorität in der Auswahl und Präsentation des Geschehens im Rahmen seiner Erzählung und stellt das Geschehen aufgrund seiner heterodiegetischen Position überwiegend ,von außen` dar (externe Fokalisierung). ${ }^{51}$ Der Erzähler beschränkt sich zumeist auf die Beschreibung des innerhalb der Fiktionswelt Sicht- und Hörbaren. Die Rollenverteilung in der literarischen ,mentalen“ Kommunikationssituation ist damit eindeutig: Es ist überwiegend Aufgabe des Erzählers, zu berichten, was geschieht, und Aufgabe des Lesers, die kausale bzw. motivationale Verbindung zwischen einzelnen Handlungsabschnitten zu erschließen. Da der Erzähler auf direkte Einblicke in die inneren Vorgänge der Figuren verzichtet, ist es dem Leser selbst überlassen, Rückschlüsse auf die Gedanken, Gefühle oder mentalen Haltungen der beschriebenen Figuren zu ziehen, wenn dies für die Interpretation einer Textpassage relevant ist. Die besondere Rolle der Erzählinstanz der Njáls Saga lässt sich vor allem an den Übergangspassagen zwischen einzelnen Szenen und Kapiteln des Werkes erkennen, die einen ersten Eindruck von den wichtigsten Erzählstrategien vermitteln. An diesen Übergängen zwischen den einzelnen semantischen Einheiten des Textes fallen die zahlreichen sich wiederholenden Phrasen und Redewendungen auf, die in leicht variierender Form als Bindeglieder zwischen den einzelnen Kapiteln stehen. Es

\footnotetext{
${ }^{51}$ Es gibt wenige, seltene Ausnahmen, die diese allgemeine Charakterisierung der Erzählsituation aufbrechen. Sie werden an späterer Stelle beschrieben und analysiert.
} 
werden sowohl einleitende, überleitende als auch abschließende Formeln verwendet, um den Übergang zwischen zwei nacheinander stehenden Episoden zu markieren und die einzelnen Ereignisse und Situationen erzählerisch zu einem Ganzen verknüpfen. Zu Beginn eines neuen Abschnittes (zumeist fällt dies mit dem Beginn eines Kapitels zusammen) stehen die in standardisierten Formeln vorgestellten Familiengenealogien. Sie beginnen in der Regel folgendermaßen: „There was a man named X, the son of Y.“ Das fünfte Kapitel der hier verwendeten englischsprachigen Übersetzung des Werkes beginnt zum Beispiel mit dem Satz: „There was a man named Atli, the son of Arnvid, Earl of East Gotland“ (NSE, S. 11). ${ }^{52}$ Neben diesen einleitenden Wendungen sind auch standardisierte Übergangsformeln zu finden, die sich hauptsächlich durch zwei verschiedene Formulierungen manifestieren, die sich im isländischen Original regelmäßig wiederholen, und für die es keine günstige wortgetreue englische oder deutsche Übersetzung gibt. Obwohl die Übersetzer dieser zwei Formeln an vielen Stellen leicht variierende Übersetzungen wählen, geht die Formelhaftigkeit dieser beiden standardisierten Wendungen nicht verloren. Dennoch seien an dieser Stelle einige Beispiele genannt, da auch sie zur Charakterisierung der Erzählsituation beitragen. Die erste der zwei verwendeten Redewendungen lautet im Altisländischen: „Nú er at segja frá [...]“ (z. B. NSI, S. 333) und wird in der englischsprachigen Ausgabe mit „Now we must tell about $[\ldots]^{“}$ (z. B. NSE, S. 260), „Now we are told that [...]“ (z. B. NSE, S. 97), und „Now to tell of [...]“(z. B. NSE, S. 157) übersetzt. Die zweite Formel, die sich im Original häufig wiederholt lautet: „Nú er par til máls at taka“ (z. B. NSI, S. 29). Hierbei handelt es sich um eine formalisierte Redewendung, die in der englischsprachigen Ausgabe ebenfalls in leicht variierender Art und Weise übersetzt wird. Hier seien nur einige Möglichkeiten, der durch den Übersetzer gewählten Übertragungen ins Englische genannt: „Now the saga continues with [...]" (NSE, S. 21), „Now it must be told that [...]" (NSE, S. 39), "Now we turn to [...]” (NSE, S. 108), "Now to return to [...]" (NSE, S. 123), "Now we go back to [...]" (NSE, S. 177), "Now we have to speak about [...]" (NSE, S. 253), "Now we take up the story of [...]" (NSE, S. 287).

Neben den ein- und überleitenden Formeln stehen dem Erzähler zusätzlich Redewendungen zu Verfügung, die eine Ereigniskette vorerst oder auch endgültig beenden. Am Ende des achten Kapitels ,entlässt` der Erzähler die zwei Figuren Hrútur und Mörður, die zuvor im Fokus der Handlung gestanden hatten, mit den Worten: „,...] og er nú lokið prætum peirra

\footnotetext{
${ }^{52}$ Es handelt sich dabei um eine nicht wortgetreue, aber inhaltlich recht nahe Übersetzung des altisländischen Originals: „Atli hét maðr; Hann var son Arnviðar jarls ór Gautlandi inu eystra“ (NSI, S. 16).
} 
Marðar“ (NSI, S. 29) aus der Erzählung. Die englische Übersetzung dieser Textstelle lautet: „[...] and that is the end of the story about Mord and Hrút” (NSE, S. 20). Eine weitere Schlussphrase, die vielfach in der gleichen oder einer leicht abgewandelten Form wiederholt wird, lautet: „[...] er ór sọgunni” Beispiele hierfür finden sich gleich zweifach in Kapitel 80, in dem es im vorletzten Absatz heißt: „Bjó Geirr goði í Hlíð til dauðadags, ok er hann ór sọgunni“ (NSI, S. 196). Diese Phrase wird in der englischen Ausgabe folgendermaßen und damit beinahe wortgetreu wiedergegeben: „Geir Godi lived at Hlíd till his dying day, and he is now out of the saga“ (NSE, S. 156). Nur einen Absatz später verabschiedet der Erzähler auch Gunnars Sohn Högni mit den Worten: „Họgni hélt vináttu sinni við Njál, ok er hann ór pessi sọgu“(NSI, S. 196). ${ }^{53}$

An den genannten einleitenden, überleitenden und abschließenden Stellen wird die aktive Rolle des Erzählers in der Gestaltung der Erzählchronologie und der Auswahl des zu erzählenden Stoffes deutlich spürbar. Der Erzähler der Njáls Saga verfügt über weitreichendes Wissen über den Stoff, in dessen Kontext die erzählten Ereignisse und Figuren stehen, und gibt seinen Wissensvorsprung und die Ausschnitthaftigkeit der erzählten Ereignisse an vielen Stellen deutlich zu erkennen. Seine umfassende Stoffkenntnis wird vor allem dann spürbar, wenn er mit seiner Wortwahl impliziert, einen Überblick über zukünftige Ereignisse zu haben, dem Leser damit also einen Blick in zukünftige Ereignisse und Entwicklungen innerhalb der Geschichte gewährt. So sagt er über Njáll und dessen Frau Bergpóra bereits bei der Einführung des Titelhelden der Geschichte: „They had six children, three daughters and three sons, all of whom play a role in this saga" (NSE, S. 40) ${ }^{54}$ Ein weiteres Beispiel ist die folgende Textstelle: „Another brother of Gunnar was Orm Skógarnef. He was illegitimate and plays no role in the saga" (NSE, S. 40). An dieser Stelle wird ein für die zu erzählende Geschichte irrelevantes Element - in diesem Fall eine Figur - nicht einfach ausgelassen. Der Erzähler nennt Orms Namen zugunsten der vollständigen Vorstellung der Familienverhältnisse und unterstreicht damit erneut die Komplexität der Familienverhältnisse, die über den Rahmen der Njáls Saga hinausgeht. Dies sind nur ausgewählte Beispiele für zahlreiche Passagen dieser Art, die auf das umfassende Wissen des Saga-Erzählers über den gesamten zu erzählenden Stoff hinweisen und sowohl der Wissensvorsprung des Erzählers

\footnotetext{
${ }^{53}$ Übersetzung: („Hogni maintained his close friendship with Njál, and is now out of the saga“ (NSE, S. 156).

${ }^{54}$ Es ist hier ebenfalls interessant zu erwähnen, dass die Bedeutung des isländischen Wortes , saga die Konnotation des Realitätsbezuges bereits in sich trägt. Wenn man diese Konnotation aufheben will, muss der Hinweis, dass die entsprechende Geschichte durch einen Dichter geschaffen wurde, dem Wort beigefügt sein. ,Erdichtete' Geschichten werden im Isländischen daher mit dem Wort ,skáldsaga' (skáld = Dichter) erfasst.
} 
als auch die Ausschnitthaftigkeit des dargestellten Geschehens geraten durch die wiederholte Verwendung solcher Formulierungen zu keinem Zeitpunkt der Lektüre in Vergessenheit. Durch diese Erzählstrategie wird außerdem die Möglichkeit geschaffen, Figuren und Ereignisse frühzeitig (in der Regel bereits im Zuge ihrer ersten Erwähnung) für den Leser in einen interpretativen Kontext zu stellen. Folgende Textstelle hebt die Bedeutsamkeit einer neu eingeführten Figur deutlich hervor, so dass gleich zu Beginn ein deutlicher interpretativer Bezugsrahmen hergestellt ist: „A son named Mord was born to Unn and Valgard; he plays an important part in this saga. When he grew up he treated his kinsfolk badly and Gunnar worst of all. He was a sly and wily fellow and the worst troublemaker" (NSE, S. 49). Mit diesen Informationen, die der Erzähler bereits bei der Erstvorstellung der Figur an den Leser vermittelt, sind das Verhalten und die Entwicklung der Figur im Verlauf der Handlung zum Teil vorhersehbar. Tatsächlich bestätigen sich Vorgriffe dieser Art durch den Erzähler im Verlauf der Erzählung zuverlässig und Mörður (Mord) wird im weiteren Verlauf der Erzählung zu einem der wichtigsten Anti-Helden der Geschichte gehören.

Diese Formeln strukturieren den Erzählfluss und bestärken damit das Gefühl, der Erzähler übernehme eine aktive Rolle in der Gestaltung des Erzählvorgangs. Diese Rolle lässt sich mit derjenigen eines ,Dirigenten“ vergleichen, der einzelne Motive und Episoden unter anderem nach seinen eigenen Gestaltungspräferenzen zu einem großen Ganzen zusammenfügt. Hier wird ein Erzähler konstruiert, der offenlegt, dass er entscheidet, welche Hinweise zu welchem Zeitpunkt vergeben und welche Handlungen an welcher Stelle berichtet werden. Obwohl es sich nicht um eine reale Person handelt, ist diese Kompetenz für den Leser allein durch die Wortwahl und die Textkomposition spürbar. Ein Beispiel soll dies illustrieren. An folgender Stelle liegt Fokus der Aufmerksamkeit zunächst auf Flosi und seiner Gefolgschaft. Anschließend unterbricht der Erzähler die Handlungsfolge und lenkt die eigene Aufmerksamkeit (und damit auch diejenige des Lesers) auf die Geschehnisse in Njálls Haus. Das voranstehende Kapitel endet mit den Worten: „The sons of Sigfús were loud in their condemnation of him, but Flosi asked them not to say anything ill about Ingjald in his absence [...]” (NSE, S. 252). Zu Beginn des darauffolgenden Kapitels steht eine der typischen rhethorischen Übergangsformulierungen, die den Fokus der Aufmerksamkeit auf einem anderen Ort und auf einer anderen Figurengruppe verschiebt: „Now we have to speak about what happened at Bergthórshvál. Helgi and Grím told their father that they would not be home that evening, and then they rode to Hólar where some foster children of theirs lived" (NSE, S. 253). 
Der Leser ist gezwungen, den sprachlichen ,Zeigegesten“ dieser Erzählinstanz auf diejenigen Momente des Geschehens folgen, die der Erzähler - so entsteht der Eindruck - bewusst und absichtlich ins Zentrum der Aufmerksamkeit stellt. Diese Situation ist mit der realen Interaktion zweier Individuen vergleichbar, im Zuge derer die Aufmerksamkeit beider Beteiligten durch bestimmte (in der Regel nonverbale) kommunikative Gesten miteinander koordiniert werden. Das Besondere an der Situation der Textlektüre ist allerdings, dass hier nicht zwei reale Personen miteinander interagieren. Vielmehr wird hier die Aufmerksamkeit des Lesers durch eine textuell generierte Instanz auf bestimmte im Text dargestellte Ereignisse gelenkt. Die interaktiven kommunikativen Strategien (v. a. nonverbale Mittel wie Zeigegesten, Mimik, Blickkontakt usw.), die im realen sozialen Kontext zu diesem Zweck häufig zum Einsatz kommen, stehen während der Textlektüre nicht in ihrer eigentlichen Form zur Verfügung. Allerdings lassen sich bestimmte sprachliche Kommunikationsstrategien im Zuge textuell vermittelter Informationen ausmachen, die die Funktion nonverbaler Kommunikationsstrategien übernehmen. Nonverbale Kommunikationsstrategien können z. B. auf der Ebene der Geschichte in der Figureninteraktion sprachlich dargestellt werden, wenn eine Figur beispielsweise auf ein Objekt innerhalb der Fiktionswelt zeigt. Im konkreten Fall der Njáls Saga ist es jedoch vor allem der Saga-Erzähler, der die Aufmerksamkeit des Lesers mit Hilfe der zuvor beschriebenen Formeln von einem Ereignis auf ein anderes umlenkt.

Eine solche Anleitung durch eine Erzählinstanz ist vor allem dann wichtig und notwendig, wenn Leser mit der Aufgabe konfrontiert sind, (z. B. mit Hilfe ihrer ToM) eigenständige Attributionen zu leisten und Handlungselemente kognitiv zu einem kohärenten Ganzen zu verbinden. Dies wird vor allem dann sichtbar, wenn die inneren Vorgänge und mentalen Haltungen von Figuren erschlossen werden müssen, um die Handlungsmotivierung zu verstehen. Wie bereits erwähnt, gewährt der Erzähler der Njáls Saga überwiegend keine direkten Einblicke in die inneren Vorgänge der Figuren. Auf diese Weise wird eine Erzählsituation geschaffen, die mit einer realen Situation und der Interaktion zweier Kommunikationspartner, die ebenfalls nur indirekten Zugang zu den inneren Vorgängen anderer verfügen, vergleichbar ist. Die Rolle des Erzählers in der Strukturierung des Stoffes und der Gestaltung des Erzählvorgangs ist dabei zwar eine aktive, sie ist dabei an die Möglichkeiten und Grenzen eines echten Menschen bzw. Geschichtenerzählers gebunden. Das bedeutet zum Beispiel, dass der Erzähler der Njáls Saga keinen ,unnatürlichen“ direkten Einblick in die Gedankenvorgänge der Figuren hat und der Erzählvorgang selbst an vielen Stellen ,spürbar` sind. Stefán Einarsson schreibt über den Erzähler der Njáls Saga: „He masters the saga technique of revealing men's minds by their outward visible reflexes“ (Einarsson, 1955, S. 151). 
Einarsson bezeichnet den Saga-Erzähler als ,supreme behaviorist‘. Diese Schlussfolgerung beruht auf der Beobachtung, dass die inneren Vorgänge und Haltungen der Figuren häufig durch den Bericht über äußerlich sichtbare Veränderungen in Mimik und Gestik oder eben in Handlungen wie den zuvor beschriebenen indiziert sind (Ebd. S. 152). Sveinsson (1954, S. 93) schreibt dazu:

$[\ldots]$ the reader is not given a constant insight into [...] minds and hearts; rather, he is given brief views or glimpses of what is going on there. And what he perceives is not a logical debate, but a blind storm of passion. It sometimes seems to the reader as if he were gazing into the twilight, where half or fully concealed forces and unknown motives are at work no less than those which are fully apparent.

Einarsson ist der Auffassung, dass - obwohl innere Vorgänge und Motive nicht in direkter Form an den Leser vermittelt werden - sie dennoch für die Motivierung der Handlungen und Figuren eine bedeutende Rolle spielen. Trotzdem keine direkten Einblicke in innere Vorgänge der handelnden Figuren gewährt werden, finden sich dennoch wichtige Hinweise darauf, die einem Leser dabei helfen, entsprechende interpretative Schlüsse zu ziehen. Ein wichtiges Mittel, mit Hilfe dessen solche Hinweise vergeben werden, ist die präzise Beschreibung mimischer und gestischer Vorgänge im Verlauf der Handlungsbeschreibung. Leser der Njáls Saga befinden sich damit in einer ähnlichen Ausgangssituation wie Menschen, die Handlungen im realen sozialen Kontext beobachten. Mimische und gestische Aktionen und Reaktionen weisen in beiden Kontexten die emotionalen Vorgänge und mentalen Haltungen der Akteure aus.

Es lassen sich zahlreiche Textpassagen der Njáls Saga identifizieren, die illustrieren, dass es sich hierbei um eine für diesen Text typische Erzählstrategie handelt. Eine solche Textpassage steht im Kontext des (später noch ausführlicher analysierten) Treffens zwischen Flosi und Hildigunnur. Die Passage lautet in der Isländischen Ausgabe: „Flosa brá svá við at hann var í andliti stundum rauðr sem blóð, en stundum fọlr sem gras, en stundum blár sem hel“ (NSI, S. 292) Hier sind ausschließlich sichtbare körperliche Signale genannt, womit die reine Beobachtungsposition der Erzählinstanz unterstrichen wird. Der letzte interpretatorische Schritt (die Rekonstruktion der inneren Vorgänge der Figur, die damit angedeutet werden) bleibt in der Isländischen Ausgabe dem Rezipienten überlassen. Interessanterweise ist die englische Übersetzung des Textes an dieser Stelle in entscheidender Weise ungenau, da sie eine direkte Vermittlung der emotionalen Vorgänge der Figur durch die Erzählinstanz suggeriert. Hier heißt es: „Flosi was so agitated that his face was now as red as blood, now as 
wan as grass, and again as black as Hell itself“" (NSE, S. 228). An einer zweiten, sehr ähnlichen Stelle, gilt das gleiche Prinzip. In der Übersetzung heißt es: „When Thórhall Ásgrímsson was told that his foster father Njál was dead [...], he was so wrought up that his entire body swelled and there burst forth from both his ears a stream of blood, and it could not be stopped. He fell in a faint, and then the flow of blood stopped" (NSE, S. 269). Im isländischen Original steht an dieser Stelle dieselbe Formulierung, wie schon im oben zitierten Beispiel: „Pórhalli Ásgrímssyni brá svá vio, er honum var sagt, at Njáll, fóstri hans, var dauðr ok hann hafði inni brunnit, at hann prútnaði allr ok blóðbogi stóð ór hvárritveggju hlustinni, ok varð eigi stọðvat, ok fell hann í óvit, ok pá stọðvaðisk” (NSI, S. 344). Auch hier gilt, dass die isländische Formulierung nur eine beobachtende Position des Erzählers suggeriert, der sich lediglich darauf beschränkt, die ,sichtbaren‘ Reaktionen der Figur zu beschreiben. Diese systematische Differenz zwischen Übersetzung und Original lässt sich an zahlreichen weiteren Textstellen zeigen, wobei in allen Fällen die Hinweise auf äußere Reaktionen durch die Interpretation des Übersetzers ersetzt zu werden scheinen. Zu Beginn des achten Kapitels der englischen Ausgabe heißt es zum Beispiel: „When Hrút came home and learned that his wife was gone, he was extremely put out. However, he controlled his feelings very well [...]“ (NSE, S. 19). Dies ist keine treue Widergabe der isländischen Textstelle: „Hrútr kom heim ok brá mjọg í brún, er kona hans var í brautu, ok var bó vel stilltr [...]“ (NSI, S. 26). Eine wortgetreue Übersetzung würde folgendermaßen lauten: „Hrútur kam nach Hause und runzelte die Augenbrauen, als er erfuhr, dass seine Frau gegangen war, doch er zeigte sich ansonsten recht beherrscht. ${ }^{\text {"55 }}$ Die subtile Differenz zwischen der englischsprachigen Übersetzung und dem Originaltext erhellt einen wichtigen Interpretationsschritt. Der Übersetzer des Textes liest Hrúturs Verärgerung an der Mimik der Figur (einem Augenbrauenrunzeln) ab und übersetzt die Passage entsprechend. Damit nimmt er dem Leser des Textes diesen Interpretationsschritt - die Zuweisung einer emotionalen Haltung aufgrund einer mimischen Geste - vorweg. Während der Leser des isländischen Originaltextes der Njáls Saga dazu angehalten ist, eine eigenständige Schlussfolgerung über die inneren Vorgänge der Figuren zu ziehen, wird ihm dieser Kognitionsvorgang hier durch den Übersetzer abgenommen. Im ersten Fall stehen ihm lediglich Hinweise über die äußeren Reaktionen der Figur und Informationen über den Handlungskontext und äußerlich sichtbare Reaktionen zur Verfügung, so dass den fiktiven Figuren gegenüber eine Interpretationssituation entsteht, die mit dem realen Kontext vergleichbar ist. Wäre der Text in dieser Hinsicht

\footnotetext{
${ }^{55}$ Meine Übersetzung.
} 
anders konfiguriert - gäbe es beispielsweise (wie im Fall der hier gewählten englischsprachigen Übersetzung) eine direkte Einsicht in die gedanklichen Vorgänge der fiktiven Figuren, wäre die Interpretation der ,sichtbaren“ Reaktionen überflüssig. Die selbstständige Attribuierungsleistung seitens des Lesers würde in einem solchen Fall entfallen, da die entsprechenden Informationen durch den Text bereits vorgegeben sind. Hier zeigt sich deutlich, inwiefern die Beschaffenheit der Informationsvergabe durch einen Text die Bedingungen für den kognitiven Rezeptionsakt während der Lektüre festlegt.

Die Beschreibung von mimischen und gestischen Vorgängen ist nur eine Strategie, einem Leser Hinweise auf innere Vorgänge zu vermitteln. Tatsächlich finden sich strukturelle Besonderheiten, die die kognitiven Interpretationsprozesse seitens des Lesers auf wesentlich komplexere Art und Weise stimuliert. Dabei steht er, um bestimmte Passagen kohärent verstehen zu können, vielfach vor der Aufgabe, Informationen zu synthetisieren, die er zu einem früheren Zeitpunkt während der Lektüre erhalten hat. Eine ausgewählte Textstelle soll dazu dienen, diese Prozesse zu beschreiben und auf diesem Weg die Bedeutung und den Zusammenhang zwischen der Informationsordnung im Text und den kognitiven Interpretationsprozessen seitens des Lesers zu beleuchten. Die Passage, die im Folgenden im Fokus der Analyse steht, umfasst das Kapitel 116 der hier verwendeten englischsprachigen Ausgabe. Den inhaltlichen Kontext für diese Textstelle bildet der Tod von Höskuldur Práinsson, der durch seine Ziehbrüder (Njáls Söhne) gewaltsam durch mehrere Schwerthiebe von verschiedenen Seiten zu Tode kommt. Mörður, einer der wichtigsten Anti-Helden der Geschichte hatte zuvor Unfrieden und Missgunst zwischen den vier Männern gesät, die ehemals eine feste und brüderliche Freundschaft miteinander verbunden hatte. Hildigunnur, die Witwe des Ermordeten, hofft auf eine Vergeltung der Tat und fordert die Blutrache durch die Hand ihres eigenen Onkels (Flosi).

Um die Informationsverarbeitungsprozesse während der Lektüre dieses Textabschnittes besser zu verstehen, ist zunächst ein Blick auf die notwendigen Vorinformationen notwendig, die der Leser benötigt - und auch erhält - um diese Szene sinnvoll und kohärent zu rekonstruieren. Die zu einem früheren Zeitpunkt der Lektüre vergebenen Informationen helfen einem Leser insbesondere dabei, die Wissensstände der beteiligten Figuren zu bestimmen. Diese sind wiederum eine wichtige Stütze für den Leser, der den handelnden Figuren an späterer Stelle darauf basierend bestimmte Intentionen und Haltungen zuschreiben kann. So erhält der Leser eine Reihe von Hinweisen am Ende des voranstehenden Kapitels (115), die das Verständnis der Szene zwischen Flosi und Hildigunnur im anschließenden Kapitel 116 
maßgeblich beeinflussen. Ein Gespräch zwischen Flosi und einer dritten Figur (Rúnólfur) gibt hier Einblick in die intentionale Haltung von Flosi, mit der letzterer später in den Dialog mit Hildigunnur tritt. Rúnólfurs berät Flosi darüber, wie er mit der durch den Mord entstandenen Rechtssituation umgehen sollte. Sein Rat lautete folgendermaßen: „Therefore, I wish to ask you, Flosi, to check your anger and decide upon that course which will lead to the least trouble, for Njál and other most excellent men are likely to make good offers [for a reconciliation]“ (NSE, S. 226). Flosi verlässt Rúnólfur mit den folgenden Worten: „Your advice will have great weight with me, unless indeed things turn out worse than they should“ (ebd.). Mit Hilfe des durch diese Aussagen gewonnenen Wissens ist ersichtlich, dass Flosi Hildigunnir mit einer friedensuchenden Intention gegenübertritt.

Auch für Hildigunnur kann der Leser eine grundlegende charakterliche Einschätzung abrufen. Da sich in unmittelbarer textueller Nähe zum Kapitel 116 keine Angaben über Hildigunnurs Absichten finden, ist es hierfür allerdings notwendig, mit Hilfe der im Verlauf der Lektüre gesammelten Informationen ein Bild ihrer charakterlichen Eigenschaften herzuleiten, auf dessen Basis sich ihre intentionale Ausgangssituation erahnen lässt. Er kann beispielsweise auf das kurze durch den Erzähler vermittelte Persönlichkeitsprofil zurückgreifen, das wesentlich früher im Text positioniert ist. Hier heißt es über Hildigunnur: „Starkad, Flosi's brother, had a daughter named Hildigunn, a beautiful and high-spirited woman. Few women were her equal in skilled handiwork. She was of a hard disposition and unforgiving, but a person who could be relied on when the occasion demanded it" (NSE, S. 191). Hier werden dem Leser wenige ausgewählte, jedoch ausschlaggebende Eigenschaften der Figur vermittelt. Diese selektive Darstellung der Persönlichkeitsmerkmale und das Kontextwissen des Lesers erleichtern die Einschätzung der Ausgangssituation in der sich Flosi und Hildigunnur gegenübertreten. Diese Situation wird von zwei konträren mentalen Haltungen bestimmt. Da der Mord an ihrem Mann aus ihrer Sicht unmotiviert und damit unehrenhaft war, und entsprechend ihrer charakterlichen Disposition, erwartet Hildigunnur, dass Flosi an ihrer statt Blutrache an Njálls Söhnen nehmen wird. Flosis vernunftgetriebener Wunsch nach einer friedlichen Einigung steht dem Wunsch seiner Nichte nach gewaltsamer Rache gegenüber. Die Hinweissituation, die dieses Verständnis der Textstelle stützt, ist ausreichend dicht. Daher ist ein kohärentes Textverständnis des Kapitels 116 gewährleistet, auch wenn hier (wie gewohnt, von außen') eine Kommunikationssituation zwischen zwei Figuren dargestellt ist, die sich durch symbolische Gesten und verschlüsselte, implizite Kommunikation auszeichnet. Sie kann mit dem Vorwissen, das der Leser aufgrund der Beschaffenheit des 
vorangehenden Textes erlangt hat und durch die der Attribution und Antizipation von mentalen Vorgängen konfliktfrei entschlüsselt werden kann.

Das Kapitel beginnt mit der Ankunft von Flosi und seiner Gefolgschaft im Hause von Hildigunnur, die ihren Verwandten mit folgenden, übermäßig warmen Worten empfängt: „Thrice welcome, kinsman! My heart rejoices at your coming!““ (NSE, S. 227). Flosi - im Vergleich zu den üblichen Begrüßungsgepflogenheiten - antwortet in unterkühlter Manier: „We shall eat our day-meal here and then ride on“ (ebd.). Schon an dieser frühen Stelle eröffnet sich eine Ebene der Sub-Kommunikation, die auf textueller Ebene verdeckt bleibt. Der Leser muss, um das ungewöhnliche Verhalten der Akteure zu interpretieren, eigene Schlüsse bezüglich der grundsätzlichen Intentionen der beiden Figuren ziehen. So lassen die ungewöhnlich herzliche auf der einen bzw. ungewöhnlich kühle Begrüßungsformel auf der anderen Seite darauf hin, dass sich beide Figuren über die Problematik der Situation und die grundsätzliche Einstellung ihres Gegenübers bewusst sind. Dieses Verständnis ist vor allem deshalb wichtig, weil die nachfolgenden symbolisch anmutenden Handlungen und Ereignisse auf dieser Basis in einen kohärenten Kontext integriert werden können. Fehlt dieses Verständnis der Ausgangssituation, dann muten die im Folgenden (gleichsam ausschließlich ,von außen ') geschilderten Handlungen und Aussagen seltsam und unmotiviert an. Der Erzähler berichtet z. B. episodenhaft davon, dass Flosi in symbolischer Anspielung auf Höskuldurs zerstochenen Umhang, ein zerrissenes Handtuch erhält, um sich die Hände zu reinigen. Er gerät daraufhin in Rage und verwendet stattdessen einen Teil der Tischdecke anstelle des Handtuchs. Daran schließt sich eine Szene an, in der Hildigunnur mit einem symbolischen Gestus Flosi den blutverklebten Umhang des getöteten Ehemannes über die Schultern legt. Auch wenn die Anspannung, die zwischen den beiden Figuren herrscht, innerhalb dieser kurzen Handlungsfolge immer wieder spürbar wird, gilt auch hier, wie für den gesamten Text der Njáls Saga, dass die inneren Haltungen der Figuren durch den Leser selbst erschlossen werden müssen. Seine Interpretation basiert lediglich auf den in die Informationsstruktur des Textes eingefassten Hinweise.

Nachdem Hildigunnurs symbolischen Hinweisen und Provokationen keine offene Stellungnahme ihres Onkels zu ihrem erlittenen Verlust und einer entsprechenden Vergeltung folgt, erfährt der Leser: „Then Hildigunn came into the room and brushed the hair from her eyes and wept" (ebd.). Flosi reagiert daraufhin mit folgenden Worten und vermeidet immer noch die explizite Benennung des im Raum stehenden Konflikts und damit die direkte Konfrontation mit seiner Nichte: ,You are sad of heart, kinswoman; that is as it should be, for your 
mourn a brave man!“ (ebd.). Diese Worte lassen darauf schließen, dass Flosi nicht vorhat, ihren erlittenen Verlust mit Blutrache zu vergelten. Der Leser kann diesen Schluss ziehen. Er muss - um die anschließenden Handlungen zu verstehen - (ohne dass dies durch die Figur oder den Erzähler ausgesprochen wird) auch den Schluss ziehen, dass Hildigunnur ebenfalls zu dem gleichen Schluss gelangt. Aus diesem Grund legt sie am Ende des Kapitels ihre Forderungen offen: „I call God and all good men to witness that I adjure you by all the wonders of your Christ and by your manhood and valour that you avenge all the wounds which Hoskuld received on his body, or else be called a caitiff wretch before all men!“ (NSE, S. 228). Erst hier spricht sie ihr Begehren gegenüber ihrem Onkel deutlich aus. Mit Hilfe der relevanten kognitiven Mechanismen ist es für den Leser jedoch kein Problem diesen Wunsch bereits im Zuge der , beobachtbaren` Handlungen zwischen Flosi und Hildigunnur zu antizipieren.

Die hier analysierte Passage veranschaulicht die Rolle der ToM, wenn literarische Handlungen durch teilweise nur symbolisch angedeutete mentale Haltungen motiviert sind. Leser, die solche impliziten Handlungsmotivationen nicht verstehen, fehlt die Grundlage zur Interpretation dieser Textpassage bis zu dem Moment, in welchem Hildigunnur ihre Intention und Beweggründe offenlegt.

\subsubsection{Parameter für die kognitive Interpretation - die Ordnung der Informationsvergabe}

Die Informationsvergabe an den Leser wird nicht allein durch die Erzählsituation bzw. die Konfiguration der Erzählinstanz bestimmt, sondern ebenfalls durch die Ordnung der geschilderten Ereigniskette. Ein Blick auf die Chronologie der Ereignisse, die in der Njáls Saga geschildert werden zeigt, dass sich der Text durch eine prospektive Darstellungsform des Geschehens auszeichnet. Die Ereignisse reihen sich überwiegend in stringent konsekutiver Abfolge aneinander. Die chronologische Abfolge der Ereignisse wird durch den Erzähler nicht umgestellt, allerdings werden verschiedene Abschnitte aneinandergereiht, zwischen denen ein größerer Sprung der Handlungszeit stattfindet.

Allerdings ist auffällig, dass Leser mit Hilfe verschiedener Mittel immer wieder auf zukünftige Ereignisse aufmerksam gemacht werden und damit - wie die Erzählinstanz - zukünftige Handlungsentwicklungen antizipieren können. Entsprechende Hinweise manifestieren sich z. B. in Form von Prophezeiungen, Träumen und Visionen einzelner Figuren. Der Moment der Vorausdeutung und der Zeitpunkt des eigentlichen Ereignisses sind in der Njáls Saga 
durch unterschiedlich lange Zeiträume sowohl in der Erzähl- als auch in der erzählten Zeit voneinander getrennt. Der Fokus der Narration richtet sich in der ,Zwischenzeit‘ häufig auf Vorgänge, die für das antizipierte Ereignis nur indirekt oder gar nicht bedeutsam sind. Das bedeutet, dass Leser - sobald sich ein einmal vorausgedeutetes Ereignis abzeichnet oder zuvor vergebene Informationen für die Interpretation des gegenwärtigen erzählten Geschehens relevant werden, die passenden Informationen aus dem Gedächtnis abrufen müssen. Die zuvor analysierte Passage zwischen Hildigunnur und Flosi (Kapitel 116) ist ein passendes Beispiel hierfür, da Leser ihre Interpretation der Szene durch Informationen stützen können, die sie bereits bei der Einführung der Figur Hildigunnurs (Kapitel 95) vermittelt bekommen hatten. Zwischen diesen beiden Kapiteln liegt der erzählerische Fokus auf der Episode der Christianisierung Islands. Leser erhalten also interpretationsrelevante Informationen, die allerdings für eine bestimmte Lektürezeit zurückgestellt und an relevanter Stelle wieder abgerufen werden müssen. Um ein weiteres Beispiel für eine solche Textstelle zu nennen, sei die folgende Passage zitiert, in der die prophetische Einordnung der Figur mit ihrer einführenden Charakterisierung kombiniert wird: „His son was Glúm, who later was to go with Flosi to the burning at Bergpórshvál" (NSE, S. 204). ${ }^{56}$ In dieser knappen Bemerkung sind zwei für den Rezipienten bedeutsame Informationen enthalten. Zum einen ruft der Erzähler an dieser Stelle dem Leser noch einmal ins Gedächtnis, dass es zu einem späteren Zeitpunkt innerhalb der Handlungsentwicklung tatsächlich zum Mordbrand in Bergpórshvál kommen wird, auch wenn dieser zum gegenwärtigen Lektürezeitpunkt aus der Handlungsmotivation noch nicht absehbar ist. Außerdem wird durch die gewählte Form der Figurenvorstellung sichergestellt, dass die ,Parteienzugehörigkeit‘ der Figur von dem Moment ihrer Vorstellung an deutlich ist. Leser können mit Hilfe dieser Information alle folgenden Handlungen der betreffenden Figur in einen interpretativen Kontext stellen. Die einführende Figurencharakterisierung ist ein auffälliges Merkmal der Njáls Saga, da sie in den meisten Fällen nach einem festen Schema strukturiert ist, welches sich regelmäßig wiederholt. Dies lässt sich am besten an einigen konkreten Textbeispielen illustrieren. Die erste Vorstellung der wichtigsten Figuren erfolgt in der Regel durch die Erzählinstanz selbst und läuft nach einem schematisierten Muster ab. Dieses beginnt mit einer einleitenden Formel ,A man was named [...], the son of [... $]^{\text {‘ }}$ und wird von detaillierteren Angaben zu familiärer Abstammung und Herkunft ergänzt. Wie sich eine solche Figureneinführung konkret darstellt, soll der entsprechende Textabschnitt zur Vorstellung des prominentesten Charakters der Saga illustrieren:

\footnotetext{
${ }^{56}$ Meine Hervorhebung.
} 
A man was named Njál, the son of Thorgeir Gollnir, the son of Thórólf. Njál's mother was named Ásgerd and she was the daughter of the chieftain Áskel the Silent. She had come out to Iceland and taken land east of the Markar River between Oldustein and Seljalandsmúli. Her son was Holta-Thórir, the father of Thorleif Crow, from whom the people of Skógar are descended, and of Thórgrím the Tall and of Skorargeir. (NSE, S. 40).

Dem Stammbaum und dem familiären Hintergrund folgt eine nähere Charakterisierung der Figur. Sie beginnt mit der Beschreibung ihrer äußeren Eigenschaften, gefolgt von Angaben zu ihren besonderen Fähigkeiten und wird zuletzt durch Hinweise bezüglich wichtiger charakterlichen Eigenschaften abgeschlossen. Diese Grundstruktur findet sich in nahezu allen Erstcharakterisierungen wieder. Die an diesen Stellen vergebenen Informationen sind im Fall jeder einzelnen wichtigen Figur augenscheinlich aus einer größeren Menge allgemeiner Eigenschaften ausgewählt und es stellt sich die Frage, warum der Erzähler seine (und damit auch die Aufmerksamkeit des Lesers) auf eben diese ausgewählten Eigenschaften richtet. Auch Leser, die sich mit den besonderen Merkmalen der Saga-Gattung nicht auskennen, können durch die stereotype Verwendung dieses Erzählmusters und den im Verlauf der Lektüre gezogenen Verbindungen zum Handlungsfortgang die Relevanz dieser Textpassagen erkennen ,lernen'. Auch wenn die Auswahl der im Fokus stehenden Charakterzüge einer Figur im Kontext ihrer Erstvorstellung nicht motiviert ist, wird schnell deutlich, welche Funktion diese Hinweise für die literarische Motivierung einzelner Handlungsstränge übernehmen. Sie sollen nicht allein ein Portrait der Figur zeichnen, sondern darüber hinaus Leser unmittelbar auf die Bedeutung dieser Figur für den Handlungsfortgang aufmerksam machen und bieten Raum dafür, ein wertendes Urteil zu vermitteln. Die gewählte Strategie ist dabei nicht komplex, subtil oder implizit, allerdings entsteht ein stark zugeschnittenes und auf die weitere Handlung abgestimmtes Portrait der Figur. Da dies für die Einführung aller wichtigen Charaktere gleichermaßen gilt, kann ein ,Lerneffekt` für den Leser eintreten. Das lässt vermuten, dass die Informationsverarbeitungseffizienz seitens des Lesers im Laufe der Lektüre steigt, weil einführend vergebene Informationen zu einer bestimmten Figur die Antizipation bestimmter Handlungsschritte erleichtern. Die Einführung der Titelfigur ist ein geeignetes Beispiel für die typische Form der Figureneinführung dieses Textes:

Njál was a wealthy man and handsome, except that he grew no beard. He was so well versed in the law that his equal could not be found anywhere. He was learned and had the gift of second sight. He was benevolent and generous in word and deed, and everything which he advised turned out for the best. He 
was gentle and noble-minded, and helped all people who came to him with their problems. (Ebd.)

Alle hier genannten Eigenschaften der Figur spielen an gegebener Stelle im Verlauf der Ereignisabfolge eine zentrale Rolle. Njálls Wohlstand, seine Eigenschaft als Gesetzeskenner und Weitsicht in der Beurteilung von Situationen und Mitmenschen sind in allen weiteren Episoden, an denen er beteiligt ist, von Bedeutung. Das gilt auch für den fehlenden Bartwuchs, der Anlass für Spott gibt, welcher wiederum zentrale Konflikte schürt (vgl. z. B. NSE, S. 66), Versöhnungen verhindert (z. B. NSE, S. 246) und auf diese Weise wichtige Teile der gesamten Handlung motiviert.

Die Bedeutung dieser Charakterisierungstechnik für die kognitive Informationsverarbeitung lässt sich an Hand der Figur Hallgerður Langbrok illustrieren, die zweifelsohne eine der wichtigsten und am meisten diskutierten Charaktere der Njáls Saga ist. Um eine Vorstellung über ihre (fiktive) Persönlichkeit zu erlangen, stehen dem Leser mehrere unterschiedliche Informationsquellen zur Verfügung. Eine der zentralen Figuren, deren Äußerungen zu dem Eindruck des Lesers über Hallgerðurs Persönlichkeit beitragen, ist ihr Onkel Hrútur. Wie in der Njáls Saga üblich, beginnt die Einführung seiner Person, mit Hinweisen zu Familienverhältnissen, dem Erscheinungsbild, körperlichen Fähigkeiten und anschließend zur Persönlichkeit:

Hoskuld lived at Hoskulsstead in Laxárdale. He had a brother named Hrút who lived at Hrútsstead. The latter had the same mother as Hoskuld, but his father was Herjólf. Hrút was a handsome man; he was tall and strong, skilled in arms, and yet of gentle disposition. He was one of the wisest of men; stern towards his enemies, but ready to help his friends in all important matters. (NSE, S. 3)

Die Erzählinstanz, in der Rolle eines unbeteiligten Beobachters, referiert anschließend eine Situation während eines Familienfestes. In diesem Kontext werden die ersten Informationen über Hallgerðurs Erscheinungsbild preisgegeben. Diese sind durchweg positiv konnotiert: „Hoskuld had a daughter named Hallgerd who was playing on the floor with some other girls. She was beautiful and tall; her hair shone like silk and was so long that it came down to her waist" (ebd.). Der Leser ist anschließend Zeuge eines liebevollen Austausches zwischen Vater und Tochter. Höskuldur bittet später um die Meinung seines Bruders über seine Tochter: „What do you think of this girl? Don't you think she’s beautiful?“ (NSE, S. 4). Die Reaktion Hrúturs, die den Eindruck über das Persönlichkeitsbild des Mädchens in eine an- 
dere - weniger positive - Richtung verschiebt, kommt auf Grund der vorhergehenden positiven Implikationen überraschend. Ihr Onkel vermeidet zunächst eine direkte Antwort. Nachdem sein Bruder ihn allerdings darauf drängt, lautet seine Antwort: „Beautiful this maiden certainly is, and many are likely to suffer for it; but I don't know whence thief's eyes have come into our kin!" (ebd.). Die hier durch ihren Onkel geäußerten Andeutungen über Hallgerðurs Rolle im Verlauf der Handlung, erweisen sich im Verlauf der Lektüre in allen Punkten als zutreffend. Zum gegenwärtigen Zeitpunkt der Handlung ist eine derartige Entwicklung der Figur jedoch nicht absehbar.

Dem Leser stehen, wenn er diese Aussage interpretieren muss, bereits alle Informationen zur Verfügung, die dafür notwendig sind, die Glaubwürdigkeit Hrúturs und seinen Stand innerhalb der fiktiven Gesellschaft der Njáls Saga einzuschätzen. Die Erzählinstanz hatte die Persönlichkeit von Hrútur selbst als ,einen der weisesten aller Männer und damit als zuverlässige Quelle von Einschätzungen über Personen und Ereignisse der Fiktionswelt vorgezeichnet. Aufgrund der Informationslage des Textes ist es dem Leser möglich, eine Einschätzung über die Zuverlässigkeit und Glaubwürdigkeit des Erzählers und der beteiligten Figuren (Höskuldur und Hrútur) zu erstellen. Diese Einschätzung gestattet wiederum eine Evaluation der gegebenen Informationen und eine der Fiktionswelt angemessenen Interpretation der neu eingeführten Figur. Es ist auf diesem Weg auch möglich, zukünftige Handlungen von Hallgerður zu antizipieren. Wäre die Informationsstruktur des Textes in wenigen Punkten anders, als es hier der Fall ist, wäre der kognitive Rezeptionsprozess entsprechend ein anderer. Vorausgesetzt ein Leser der Njáls Saga ist nicht mit den Gattungsmerkmalen von Saga Texten vertraut und würde die Erzählinstanz als unzuverlässig einstufen, oder gesetzt den Fall Hrúturs herausragende gesellschaftliche und persönliche Stellung wäre dem Leser nicht bekannt, wäre der Interpretationsrahmen entsprechend ein anderer. Wäre Hrútur eine ungeeignete Quelle für eine Einschätzung von Hallgerðurs Eigenschaften oder wäre seine eigene Persönlichkeit erst an späterer Stelle beschrieben worden, erhielte das Persönlichkeitsbild des Mädchens zunächst einmal ein leicht abgewandeltes Profil. Der Kommentar ihres Onkels könnte möglicherweise anderen Intentionen, wie zum Beispiel einer Verleumdungsabsicht zugeschrieben werden.

Die Ordnung der Informationsvergabe innerhalb eines Textes beeinflusst hier in entscheidender Art und Weise die Verstehensprozesse seitens des Lesers. Genauer gesagt: Durch die Ordnung, Art und Menge der im Text gegebenen Informationen werden die Interpretations- 
möglichkeiten, die einem Leser für eine sinnvolle Interpretation zur Verfügung stehen, eingegrenzt. Um zu bestimmen, welche Interpretationsmöglichkeiten einem Leser offenstehen, ist es wichtig, die Informationsstruktur eines Textes zu untersuchen. Von dieser Struktur hängt $\mathrm{ab}$, welche Informationen in welcher Form in den gegenwärtigen Rezeptionsprozess und das Textverstehen des Lesers einfließen können. Über die Njáls Saga lässt sich sagen, dass sie dem Leser sowohl hinsichtlich der vorausdeutenden Darstellungsform als auch der stereotypen Figurencharakterisierung zahlreiche explizite und implizite Interpretationsrahmen vorgibt. Gleichzeitig sind Leser dieses Werkes dazu angehalten, innere Vorgänge und mentale Haltungen aufgrund der externen Fokalisierungssituation und der überwiegend geringen narrativen Distanz eigenständig zu attribuieren.

\subsection{Besondere kognitive Leistungsanforderungen in der Njáls Saga}

\subsubsection{Offene und geschlossene Handlungsmotivierung}

An einigen wenigen Textstellen ist das gerade vorgestellte Grundprinzip der Figureneinführung abgewandelt. Kári zum Beispiel - der zu den Hauptcharakteren der Saga gehört - tritt inmitten eines Handlungssegmentes (in diesem Fall einer Seeschlacht) und nicht zu Beginn eines Handlungsabschnittes auf. Auch die Erzähltechnik ist hier eine andere, denn er wird nicht ausschließlich durch die ,Stimme“ des Erzählers beschrieben, sondern stellt sich selbst vor. Auch hier fällt es dem Erzähler zu, ein erstes Bild zu vermitteln: ,[...] and at the mast of the foremost ship there stood a man wearing a silken jacket and a gildet helmet. His hair was long and fair and in his hand he carried a spear inlaid with gold" (NSE, S. 162). Position, Kleidung und Ausstattung des (noch namenlosen) Fremden sowie seine Flotte von ,[...] no

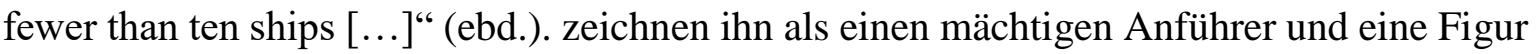
mit positiver ethischer Besetzung aus. Von Kári selbst erfährt der Leser dann Näheres über die eigene Identität und Abstammung. ${ }^{57}$ Der Erzähler bleibt dem Leser eine explizite Auskunft über die Persönlichkeit, die besonderen Fähigkeiten oder ein explizites ethisches Urteil (wie im zuvor zitierten Beispiel der Vorstellung von Njáll) schuldig. Die Attribute, mit denen diese Figur eingeführt wird (Aussehen, Kleidung, schmückende Bewaffnung, Position auf dem Schiff etc.) sind durchweg positiv konnotiert. Wie im Fall von Njáll ist damit auch hier ein Interpretationsrahmen gesetzt. Dieser wird jedoch nicht explizit ausgesprochen, sondern

\footnotetext{
${ }^{57}$ Vgl. NSE, S. 163.
} 
durch die Beschreibung der Figur, vermittelt‘. Dabei dienen Kontextinformationen und die Hinweise über , sichtbare“ Handlungen und Reaktionen der Figur (ähnlich wie im realen Leben) als Informationsquelle zur Erstellung eines Charakterprofils. Leser müssen hier eigenständige Schlüsse ziehen und die hergeleiteten Eigenschaften zu einem Werturteil über das Charakterbild dieser Figur zusammenzufügen. Hierfür ist neben den einführend gegebenen Informationen auch die Bewertung von Káris nachfolgenden Handlungen relevant.

Eine dritte Strategie der Charaktereinführung, die in der Njáls Saga vorzufinden ist, lässt sich an Hand der Erstvorstellung des ,Übeltäters“ Hrappur illustrieren. In diesem Fall erhält der Leser keine initiierenden Informationen über die Figur durch den Erzähler. Vielmehr dient ein Dialogpartner (Kolbeinn) als motivierendes Element zur Charakterisierung durch die Figur selbst. Kolbeinn spielt keine weitere Rolle innerhalb der Handlungsfolge der Njáls Saga und wird dementsprechend auch nur spärlich und oberflächlich charakterisiert. Der Leser bekommt keine expliziten Hinweise auf dessen eigene Persönlichkeit oder seine speziellen Fähigkeiten. Einzig seine Identität, Abstammung und Informationen über seine Reiseroute sind notwendig, um das Aufeinandertreffen mit Hrappur schlüssig zu begründen. Kolbeinn initiiert den Dialog, indem er um Auskunft bezüglich Namen, Abstammung und den Intentionen des Fremden bittet:

,My name is Hrapp', he answered.

,What do you want of me?' asked Kolbein.

,I want you to transport me overseas, ' he answered.

,Whose son are you?' asked Kolbein.

Hrapp answered: ,I am the son of Orgumleidi, the son of Geirólf Warrior. $[\ldots]$

,I am thinking,' said Kolbein, ,that whoever takes you along will rue it! ‘58

Die Prophezeiung am Ende dieser kurzen Passage wirft ein erstes negatives Licht auf Hrappur und kündigt dessen zukünftige Missetaten und seine durchweg negative Rolle im gesamten Handlungsverlauf an. ${ }^{59}$ Damit ist erneut ein expliziter Interpretationsrahmen für den Leser geschaffen. Dieser wird allerdings hier nicht durch den Erzähler direkt, sondern durch eine der an der Handlung teilnehmenden Figuren benannt. Kolbeinn wird somit zum ,Sprachrohr` für die Vermittlung der relevanten Informationen. Diese Erzählstrategie für die

\footnotetext{
${ }^{58}$ NSE, S. 167.

${ }^{59}$ In manchen englischsprachigen Ausgaben (auch in der hier verwendeten Ausgabe) wird dieser Effekt durch die Wahl der Kapitelüberschrift: The Blackguard Hrapp and his Misdeads in Norway zusätzlich verstärkt. Die originalsprachige Ausgabe kommt jedoch ohne Kapitelüberschriften aus. Hier bezieht der Leser ausschließlich die beschriebene Information aus dem Haupttext.
} 
Einführung neuer Charaktere ist in der Njáls Saga häufig zu finden. In solchen Passagen wird - wie bereits im Fall von Hrappur erläutert - die Informationsvergabe durch die Erzählinstanz an eine der fiktionsinternen Figuren delegiert. Diese Figuren dienen als ,Sprachrohre` zur Vermittlung eines Persönlichkeitsprofils der neuen Figur. Diese Abänderung der Erzählweise geht mit einer Veränderung der Informationsvergabe an den Leser einher. Wenn Informationen nicht mehr durch einen, übergeordneten` Erzähler, sondern eine fiktionsinterne Figur vermittelt werden, müssen Leser die so erhaltenen Informationen erst qualitativ beurteilen. Diese Bewertung steht - wie im Rahmen realer sozialer Interaktion auch - in einem engen Bezug zur Bewertung der Aussageintentionen und Charaktereigenschaften der direkten Informationsquelle. Leser benötigen für ihren Interpretationsschluss an solchen Stellen weitere Informationen, die die Stellung und Eigenschaften der fiktiven Informationsquelle betreffen und z. B. ein Urteil darüber ermöglichen, inwiefern der jeweilige Informationsgeber als zuverlässig einzustufen ist und inwiefern dessen Motive und Intentionen Einfluss auf seine Kommunikation nehmen. Erst dann können die gegebenen Hinweise in die Bewertung der neu eingeführten Figur einfließen. Innerhalb der Vergabeordnung der textuellen Informationen muss deshalb idealerweise sichergestellt sein, dass zunächst ausreichend Informationen über Figur, die als Informationsquelle dient, vorhanden sind. Wenn ein Leser zu einer bestimmten Einschätzung über die Informationsquelle gelangt ist, kann er die durch sie vergebenen Hinweise relativieren, und so kohärent in das Situationsmodell einfügen.

Der durch die (überwiegend einführend geleistete) spezifische Charakterisierung der fiktiven Figuren bietet einen starken Interpretationsrahmen und somit eine wichtige Orientierungshilfe für den Leser des Textes. Gegebene Textstrukturen lenken hier die Verstehensund Interpretationsprozesse eines Lesers, indem sie sie eingrenzen. Wenn sichergestellt ist, dass Leser ausreichend Informationen über eine bestimmte Figur oder eine bestimmte Situation besitzen, dann ist außerdem die Grundlage für die eigenständige Rekonstruktion psychologisch motivierter Handlungen gelegt. Tatsächlich ist in vielen Passagen der Njáls Saga die eigenständige Attribution von Intentionen und Emotionen durch den Leser für ein kohärentes Textverständnis unerlässlich. Das ist deshalb der Fall, weil solche mentalen Haltungen an vielen Stellen eine wichtige Funktion in der Motivierung des Handlungsfortgangs übernehmen, allerdings nur sehr selten direkt benannt sind. Doch ist die Handlungsmotivierung in der Njáls Saga durchgängig geschlossen? Stefán Einarsson zumindest stellt dies in Frage und schreibt: „One can be in doubt why Gunnar changes his mind and returns home to face sudden death, or why Flosi gets angry because of Njálls baugbak and rejects reconciliation [...]" (Einarsson, 1955, S. 152). Die Ambivalenzen, die Einarsson hier nennt, lassen 
sich mit Hilfe von motivierenden mentalen Haltungen, die Leser aufgrund der Informationssituation des Textes rekonstruieren können, auflösen. Gunnar wird aus seiner Heimat durch ein rechtsgültiges Urteil verbannt und folgt zunächst dem Rat seines Freundes Njáll, dieses Urteil zu respektieren. Er ändert seine Meinung nach einem Blick zurück auf seine Heimat, der er sich zu verbunden fühlt, um sie zu verlassen.

When he was ready to leave he kissed them all goodbye; [...] He took his halberd, thrust it into the ground, leaped upon his horse, and rode away with Kolskegg.

As they were riding down toward the Markar River Gunnar's horse stumbled and threw him. He looked up toward the slope where stood the farmhouses of Hlídarendi and said: 'Fair is the slope, fairer it seems than I have ever seen it before, with whitening grain and the homefield mown; and I shall ride back home and not go aboard at all.' (NSE, S. 146)

In einem anderen Kontext fühlt sich Flosi durch das Geschenk eines Seidenschals in seiner Männlichkeit angegriffen, wird außerdem durch Njálls ältesten Sohn weiterhin provoziert und lehnt die rechtliche Einigung daraufhin - verständlicherweise, so scheint es - ab:

Flosi went to the Court of Laws to look at the money and he said: 'This is a large amount of good silver, and well paid out, as was to be expected.'

Then he picked up the cloak and asked who had contributed that, but no one answered. Again he waved the gown about, laughing, and asked who might have contributed it, but still no one answered. Then he asked: 'Is it that no one of you knows who owned this piece of apparel, or is it that no one dares to tell me?'

Skarphedin spoke: 'Who do you think gave it?'

Flosi answered: 'If you must know, I'll tell you. I believe it was your father, the "Beardless One"; for many don't know, looking at him, whether he is a man or a woman!' [...]

Thereupon Skaprphedin possessed himself of the gown and tossed a pair of blue breeches at Flosi and said he needed those more.

Flosi asked: 'Why should I need those more?'

Skarphedin answered: 'Because if you are the mistress of the troll at Svínafell, as they say, then he uses you like a woman every ninth night!'

Then Flosi gave the money a kick and said he would not have a penny of it [...]. (NSE, S. 245 f.).

Der Text ist an den genannten Stellen so strukturiert und enthält solche Hinweise, dass ein Leser durch die Attribution mentaler Haltungen zu einem kohärenten Interpretationsurteil gelangen kann, auch wenn dieses durch den Text nicht explizit vorgegeben ist. Auch wenn 
individuelle Interpretationsurteile zwischen einzelnen Lesern variieren können, ist an solchen Stellen dennoch die kausale und motivationale Abfolge der Ereignisse sinnvoll und in sich geschlossen, wenn Leser mit Hilfe ihrer ToM die passenden Verknüpfungen eigenständig herstellen.

Im Verlauf der Lektüre der Njáls Saga fällt jedoch eine Textpassage auf, die sich einer kohärenten und eindeutigen Auslegung zu entziehen scheint. Gemeint ist das ,Dilemma“ des Rechtsgelehrten Eyjólfur. Es steht im Kontext des Rechtsstreites um den Mordbrand, angestiftet durch Flosi und seine Gefolgsmänner, durch den Njáll zusammen mit seiner Familie ums Leben kommen. Im Anschluss an seine Tat drohen rechtliche Konsequenzen und Flosi sucht nach seiner Flucht nach rechtlichem Rat und Beistand. Zu Beginn der Textpassage wird eine neue Figur (der Rechtgelehrte Eyjólfur) in gewohnter Manier vorgestellt, wobei der Erzähler (ebenfalls wie gewohnt) ausschließlich die signifikanten Wesensmerkmale hervorhebt:

Eyjólf was a highly respected man and so well versed in the law that he was counted one of the three greatest lawyers in Iceland. He was a very handsome man, tall and strong, and he gave every promise of becoming an eminent chieftain. Money had a great attraction for him as for other kinsmen of his. (NSE, S. 283).

Drei Punkte scheinen für das Persönlichkeitsprofil der Figur besonders wichtig zu sein. Zum einen besitzt er offenbar eine besondere Begabung als Rechtsgelehrter. Außerdem stehen seine deutlich positiv konnotierten physischen Eigenschaften im Vordergrund. Hinzu kommt eine latent negativ konnotierte Schwäche für Materielles, die hier die Charakterisierung dieser Figur abschließt. Anschließend an dieses kurze Portrait setzt der Erzähler den Bericht über die Handlung fort und der Leser erfährt u. a., dass Flosi den Rat erhält, für den ihm drohenden Prozess nach einem erstklassigen Anwalt Ausschau zu halten. Er erhält dann von demselben Ratgeber (Bjarni) den Hinweis, sich an Eyjólfur zu wenden, der den Ruf genießt, der , beste Anwalt der westlichen Gemeinden “ (NSE, S. 284). ${ }^{60} \mathrm{zu}$ sein. Andererseits ist dem Leser ebenfalls bereits bekannt, dass das Recht in diesem Fall auf der Seite der Kläger steht, die eine angemessene Entschädigung oder Vergeltung für die Brandstiftung fordern. Schon zu einem früheren Zeitpunkt während der Lektüre, im Kontext der Hatz auf Gunnar - die in dessen Tod endete - haben Leser erfahren, dass eine Brandstiftung als unehrenhafte und illegale Vorgehensweise abzulehnen ist (NSE, S. 150). Es handelt sich demnach um keinen

\footnotetext{
${ }^{60}$ meine Übersetzung
} 
Prozess, der dem Anwalt der Beschuldigten Aussicht auf Erfolg oder gar zu erlangenden Ruhm verspricht. Die intuitive Einschätzung, dass es aus diesem Grund schwierig sein wird, einen geeigneten Verteidiger zu gewinnen, teilen Erzählinstanz und die Figuren, die sich dazu äußern und über die weitere Vorgehensweise beratschlagen, gleichermaßen. Schließlich steht der Beschluss, sich mit dem Anliegen an Eyjólfur zu wenden. Bjarni wendet sich in höflicher Form mit einem allgemeinen Hilfegesuch an den Rechtsgelehrten: „It is you whom we have come to see, friend, for we are sorely in need of your help in every respect" (NSE, S. 285). Bjarni spricht an dieser Stelle nicht explizit aus, um welche Unterstützung es ihm geht. Dennoch wehrt Eyjólfur unmittelbar und mit vordergründiger Bescheidenheit ab: „There are a great many excellent men to choose from here at the Assembly, and it will be an easy matter for you to find men who will be of greater help to you than I can be" (ebd.). Aufgrund der bereits bekannten Informationen lässt sich hier erahnen, dass andere Gründe, als die vorgeschützten, der ablehnenden Grundhaltung des Rechtsgelehrten unterliegen. Eyjólfur weiß, dass die Rechtslage für den Angeklagten schwierig ist und er sich daher wenig Ruhm und Profit versprechen darf. Der Verteidiger des unehrenhaften Mörders des angesehensten Mannes der Gemeinschaft muss möglicherweise selbst eine Rufschädigung fürchten.

Um zu dieser Einschätzung zu gelangen, muss ein Leser - z. B. mittels einer Einschätzung des Wissensstandes der Figur - in der Lage sein zu erkennen, dass Eyjólfur bewusst ist, mit welchem Anliegen sich Flosi an ihn wenden wird. Daraufhin gilt es zu erkennen, dass es im Rahmen dieser spezifischen Konstellation nicht allein die Bescheidenheit Eyjólfurs sein kann, die ihn dazu veranlasst, dem noch nicht erstellten Auftrag ausweichen zu wollen. Es handelt sich schlicht um einen äußerst schwierig zu gewinnenden Rechtsstreit, dessen ethische Bewertung deutlich gegen die Aufnahme der Verteidigung spricht. Da seit der Einführung des Charakters einige Handlungszeit verstrichen ist, werden die Eigenschaften Eyjólfurs durch eine Erwiderung Bjarnis wieder aus dem Arbeitsgedächtnis abgerufen: "No, that is not so! You have many qualities in which no man here at the Assembly can surpass you" (ebd.). Da der Leser bereits durch den Erzähler eingeweiht wurde und über die Schwächen der Figur informiert ist, kann er an dieser Stelle darauf schließen, dass Bjarni dem Rechtsgelehrten hier lediglich schmeicheln möchte. Die ,mentale“ Kommunikationssituation zwischen dem Sprecher und den Eingeweihten (darunter der Leser selbst) eröffnet hier eine als zynisch oder ironisch zu bezeichnende Ebene, auf der auf diejenigen Charakterzüge des Rechtsgelehrten angespielt wird, die ihn tatsächlich zum, idealen` Ansprechpartner für Flosi und Bjarni macht, nämlich seine Schwäche für Materielles. Auf diese Interpretationsschritte 
aufbauend ist der Rezipient nun in der Lage die nachfolgende Handlung, die durch diese Ausgangssituation motiviert wird, richtig einzuordnen - ein Vorgang, der ohne die kognitiven Ressourcen der ToM nicht möglich wäre. Die entscheidende Textstelle, die die direkte Konfrontation zwischen Eyjólfur und Flosi beschreibt und deren Auslegung verschiedene Interpretationsansätze provoziert, soll an dieser Stelle zu Gunsten eines besseren Verständnisses vollständig wiedergegeben werden.

Der Vertraulichkeit des dargestellten Gespräches geschuldet, findet sich Eyjólfur dabei in einiger Entfernung von seinem eigenen Gefolge allein wieder und wird mit dem nun explizit formulierten Anliegen Flosis, der hingegen von zweien seiner Gefolgsmänner begleitet wird, konfrontiert:

'you speak handsomely,' said Eyjólf, 'but I don't propose to have anything to do with any business of yours.'

Flosi said: 'It is hardly necessary to point out what we have in mind. We wish to ask you to support us in this business of ours, go to the court with us, and seize on any points of defence, if any present themselves, plead them in our name, and stand by us at the Assembly in everything which may come to pass.'

Eyjólf started up in a fury and said that no man should presume to have him run a fool's errand or use him as a cat's-paw to do what he did not want to 'and now I see', he said, 'what was the real reason for those fine words which you spoke to me!"

Hallbjorn the Strong took hold of him and made him sit down between Bjarni and himself and then said: 'No tree falls at the first blow, friend; so just sit there a while with us.

Flosi then drew a gold ring from his arm and said: 'I wish to give you this ring, Eyjólf, for your friendship and help, and in order to show you that I will not play false. You can well afford to accept this ring, because there is no man here at the Assembly to whom I have ever given such a gift.'

The ring was so large and well made that it was worth twelve hundred yards of brown-striped homespun. Hallbjorn placed the ring on Eyjólf's arm.

'Since you behave so generously it is only reasonable of me to accept the ring, ' said Eyjólf. 'And you can also take it for granted that I shall take up the defence in your case and do everything that is required.' (NSE, S. 286).

Eine mögliche Interpretation dieser Textstelle lautet, dass der Ausgang dieser Szene auf der Bestechlichkeit Eyjólfurs beruht, der sich durch ein Wertgeschenk zu der Aufnahme einer beinahe aussichtslosen Verteidigung Flosis überreden lässt, der für den Mord an einer der angesehensten Familien verantwortlich ist. Allerdings ist auf den zweiten Blick auch das Moment der latenten Drohung durch Hallbjörn und die eher alternativlose Situation des 
Rechtsgelehrten schwer zu übersehen. Der modernisierte isländische Originaltext gibt eine Formulierung vor, die zweifelsfrei eine aggressive Konnotation mit sich bringt. Sie lautet: "Hallbjǫrn hin sterki preif til hans ok setti hann niðr í millum peira Bjarna [...]" (NSI, S. 367). ${ }^{61}$ Flosi bietet Eyjólfur in offener Freundlichkeit ein wertvolles Geschenk an, um ihn zu einer positiven Entscheidung zu bewegen, und Eyjólfur akzeptiert den Auftrag.

Um diese Passage zu verstehen, müssen Leser mit Hilfe der ToM einige wichtige Informationen, wie die Wissensstände und situationsbedingten Positionen der Figuren attribuieren. Die gegebene Informationsstruktur lässt dabei Raum für zwei unterschiedliche Auslegungen, die sich durch die Möglichkeit zur Attribution zweier unterschiedlicher motivierender Intentionen ergeben. Die erste Hälfte des zitierten Abschnittes lässt dabei keinen Zweifel an Eyjólfurs initialer Haltung gegenüber dem Anliegen der Beklagten. Flosis einleitenden Worte ,It is hardly necessary to point out what we have in mind' rufen dabei nicht nur ins Gedächtnis, dass Eyjólfur bereits erahnen müsste, um was für ein Anliegen es sich handelt. Der Leser weiß dank der bereits zuvor erhaltenen Informationen über die Intentionen des Sprechers (erste Stufe) und über das Bewusstsein der Figur über die Intention des Sprechers (zweite Stufe) Bescheid.

Die ambivalente Auslegung dieser Szene kommt zustande, weil zwei unterschiedliche Motivationen für die Meinungsänderung Eyjólfurs angeboten werden, die beide Gültigkeit besitzen, wobei der Text keine der beiden Varianten ausdrücklich verbietet oder erfordert. Es ist einerseits möglich, die positive Entscheidung des Rechtsgelehrten auf dessen Schwäche für Materielles zurückzuführen, die Flosi sich an dieser Stelle zu Nutze macht, indem er einen goldenen Ring als Geschenk überreicht. Andererseits ist die latente Bedrohung durch Hallbjörn, die durch die Wahl der Formulierung bei der Beschreibung der Szene durch die Erzählinstanz gewählt wurde, ebenfalls spürbar. Auf diese Weise wird dem zu Beginn des Kapitels durch den Erzähler vermittelten Charakterprofil Eyjólfurs vollständig Rechnung getragen, das zwar von dessen Charakterschwäche Zeugnis abgelegt hatte, ihn allerdings nicht als niederen Charakter auszeichnete. Ebenso offen bleibt die Frage, warum Eyólfur den Auftrag, die Brandstifter vor dem Gesetz zu verteidigen, annimmt. Der Zweifel oder die interpretative Unsicherheit, die ein Leser empfindet und die Einarsson benennt, lässt sich dadurch begründen, dass die Njáls Saga an vielen Stellen die eindeutige Zuweisung mentaler

\footnotetext{
${ }^{61}$ Meine Übersetzung der Textstelle: Hallbjörn der Starke packte ihn und setzte ihn zwischen sich und Bjarni.
} 
Haltungen durch bewusst von einem Autor platzierte oder z. B. durch die Texteditiongeschichte zu erklärende Ambivalenzen gegensätzlicher Informationen stört.

Ambivalente Interpretationsmöglichkeiten sind kein Alleinstellungsmerkmal der Njáls Saga. Dennoch lässt sich an dieser Stelle festhalten, dass Leser dieses Textes nicht nur zur aktiven Attribution mentaler Haltungen angehalten sind. Bedingt durch die Beschaffenheit des Textes sehen sich Leser an der beschriebenen Stelle ebenfalls damit konfrontiert, dass der Text zwei unterschiedliche Interpretationsmöglichkeiten gestattet. Es ist möglich, den unmittelbaren Ausgang der Situation (Eyjólfur nimmt das Mandat an, dass er zunächst abgelehnt hatte), durch unterschiedliche intentionale Haltungen motiviert zu sehen. Ist Eyjólfur schlicht bestechlich und damit moralisch zu verurteilen? Oder ist ihm zumindest teilweise zugute zu halten, dass er sich durch Hallbjörn körperlich bedroht sieht und damit gegebenenfalls keine andere Wahl hat? Es ist an dieser Stelle aus Sicht eines modernen Lesers sicher sinnvoll zu fragen: Ist es eine Kombination aus beiden Faktoren, die den Ausgang der Situation unausweichlich macht? Doch erstere Auslegung wurde durch die einführende Beschreibung der Figur durch den Erzähler gestützt - doch warum sollte dann zusätzlich eine bedrohende Situation geschaffen werden, um den Rechtsgelehrten zu überzeugen? Soll das negative moralische Urteil über Eyjólfur hier abgeschwächt werden, das mit einer eindeutigen Bestechung einher ginge? Diese Fragen sind basierend auf der Informationssituation des Textes nicht eindeutig zu beantworten.

Allerdings drängt sich bereits an dieser Stelle das vorläufige Fazit auf, dass die ToM induzierte Attribution motivierender mentaler Haltungen für die Njáls Saga an vielen Stellen eine wichtige Rolle spielt. Es ist in vielen Fällen der ToM-Fähigkeit des Interpreten geschuldet, dass sich basierend auf der überwiegend chronologisch und ohne direkte Hinweise auf die mentalen Haltungen der handelnden Figuren erzählten Abfolge von Ereignissen ein motivierter, kohärenter Handlungsbogen rekonstruieren lässt. Dabei finden sich Leser in einer Kognitionssituation wieder, die mit der Beobachtung relater Ereignisse vergleichbar ist. Die Geschehnisse werden von außen, ohne direkte Innensicht wahrgenommen/erzählt und es bleibt dem Beobachter/Leser überlassen, die Handlungsmotivation basierend auf den gegebenen Hinweisen zu rekonstruieren. Gleichzeitig bietet der Text an der hier im Fokus stehenden Stelle einen ambivalenten Interpretationsrahmen für die Auslegung der Passage. Ein Leser kann diese interpretatorische Offenheit akzeptieren. Er kann sich aber auch die Frage stellen, aus welchem Grund der Text so strukturiert ist, wer er ihm vorliegt. Liegt dieser Struktur ggf. eine Autorenabsicht zugrunde? Oder: Ist die gegebene Struktur im Kontext der 
Geasmtmotivierung des Textes notwendig? In diesem speziellen Fall zum Beispiel: Lässt sich rechtfertigen, dass ein moralisch einwandfreier Mann die Verteidigung einer so eindeutig verwerflichen Tat übernimmt lediglich aufgrund einer körperlichen Bedrohung übernimmt? Wenn ja, ist es im Gesamktkontext sinnvoll, Flosi damit als den ultimativen AntiHelden auszuweisen? Schließlich sind auf allen Seiten (auch durch Njáls Familie) bereits zuvor Unrecht getan worden. All diese Fragen stellen sich - mit anderen Worten: diese zusätzliche interpretatorische Komplexität entsteht durch die subtile Gegenüberstellung zweier verschiedener und entgegengesetzter motivierender Handlungsimpulse, die hier gegeben werden.

\subsubsection{Komplexe Attribution}

Ich möchte mit der Analyse eines letzten Fallbeispiels untersuchen, welche Rolle verschiedene Strategien der Darstellung von interpsychologischen Vorgängen, die mit der ToM in einen Zusammenhang zu stellen sind, für den kognitiven Rezeptionsprozess der Njáls Saga spielen. Techniken wie die explizite Reflektion über mentale Vorgänge bis zur Darstellung ToM-abhängiger sozialer Interaktionen sind dabei ebenso interessant wie die Konstruktion einer erzählerischen Grundhaltung, die den Einsatz ToM-gebundener kognitiver Aktivität ausdrücklich erfordert.

In den Textabschnitten, die die Kapitel 22 und 23 der hier verwendeten englischsprachigen Ausgabe der Njáls Saga umfassen, wird ein umfassendes Täuschungsmanöver geschildert, das zunächst (in Kapitel 22) im Stadium der Planung aus der Perspektive der beiden Figuren Njáll und Gunnar beschrieben wird. In Kapitel 23 wird anschließend die zuvor sorgsam geplante und vorhergesagte Handlung geschildert. Darstellungen von Täuschungsszenen sind deshalb besonders interessant, weil hier ToM-relevante kognitive Prozesse auf der Ebene der Geschichte nachgezeichnet werden, die sich in den Interpretationsprozessen des Lesers spiegeln. Im Fall von Täuschungen werden sich widersprechender Überzeugungen über die Welt initiiert: (A ist der Fall, Y weiß, dass A der Fall ist. X denkt, B sei der Fall, da Y möchte, dass X denkt, B sei der Fall). Wenn eine Täuschungssituation durch einen literarischen Text evoziert wird, muss ein Leser allen beteiligten fiktiven Figuren adäquate Überzeugungshaltungen, Intentionen und Wissensstände zuschreiben, um sie kohärent nachvollziehen zu können. Diese müssen zunächst durch die fiktiven Figuren (auf erster, fiktionsinterner Stufe) aber auch durch den Leser (auf zweiter, fiktionsexterner Stufe) entschlüsselt werden. Es ist 
davon auszugehen, dass diese interpretativen Vorgänge auch bei der Gestaltung der Erzählung (Ebene 0) zumindest unbewusst, im manchen Fällen aber auch bewusst berücksichtigt wurden.

Die beiden Kapitel, die im Folgenden im Fokus stehen sind u. a. deshalb spannend, da sie eine solche Täuschungssituation aus unterschiedlichen Perspektiven und mit unterschiedlichen narrativen Strategien erfassen. Ich möchte prüfen, welche besonderen kognitiven Anforderungen innerhalb dieses Lektüreabschnittes für den Leser entstehen.

Gunnar bittet seinen Freund Njáll um Rat in der Angelegenheit von Unnurs ausstehender Mitgift. Um diese Mitgift, die Hrútur ihrem Vater nach der Scheidung schuldig geblieben war zurück zu gewinnen, soll Gunnar Hrútur mit einem gewandten Manöver eine falsche Identität (,Héðinn“) vortäuschen und sein Gegenüber so zum gewünschten Verhalten bewegen (Hrútur wird den Aufruf formulieren, mit dem er selbst von Gunnar anschließend vor Gericht zitiert wird). Besonders interessant - vor allem im Vergleich mit dem eher knappen Darstellungsstil anderer Szenen in diesem Werk - ist die auffällig detaillierte Ausarbeitung der Szene über zwei Kapitel hinweg. Njáls Rat an Gunnar geht mit einer teilweise wortgetreu formulierten Vorhersage der avisierten Handlungsentwicklung und des zukünftigen Gespräches zwischen Gunnar und Hrútur einher. Er geht sogar so weit, die innere Haltung des getäuschten Hrútur vorherzusagen: „Hrút will laugh at this and think it great fun“ (NSE, S. 43) ${ }^{62}$ Ein Auszug soll die Erzählstrategie dieser Textpassage noch deutlicher veranschaulichen:

You must greet Hrút, and he will receive you well. He will ask you whether you are from the north, and you shall say you are from the Eyja Firth district. He will ask you whether there are many outstanding men there, and you shall answer that there are miserable fellows there and plenty of them. ,Do you know Reykjadale?' he will ask, and you must answer: ,I know all Iceland“ [...] Then you will talk about the Rangá District, and you must say there above all is a dearth of prominent men, now Mord Fiddle is dead. He will ask how you explain that no one could fill his place, and you must answer that he was so wise a man and so skillful in prsecuting cases that there never was any question about his leadership. He will ask: ,Do you per chance know how matters went between him and me?' ,Yes, I know, you must say, ,that he took your wife from you and you did nothing about it." Then Hrút will answer: ,Don't you think that was some disgrace for him that he didn't get her property back, though it was he himself who filed suit for it?' ,That is easily answered,' you must say. ,You challenged him to single combat; but he was an

\footnotetext{
${ }^{62}$ Meine Hervorhebung.
} 
old man, and his friends advised him not to fight against you, and thus the suit was dropped.' ,Yes, I did challenge him,' Hrút will say, ,and to stupid people that seemed lawful enough, but actually the suit could have been taken up again at another Assembly, if he had had the courage.' (NSE, S. 43 f.)

Die narrative Strategie, die hier zum Einsatz kommt, ist deshalb interessant, da für den Leser auf der eigentlichen Handlungsebene eigentlich ein Gespräch zwischen Gunnar und Njáll zu rekonstruieren ist. Diese Situation wird durch Njálls Vorhersage und die wortgetreu zitierten Repliken der zukünftigen Situation durch das eigentlich zukünftig stattfindende Gespräch zwischen Gunnar und Hrútur quasi überschrieben. Trotz der wörtlichen Widergabe der Szene zwischen Gunnar und Hrútur bleibt aber Njáll (gemäß der Erzählsituation in dieser Passage) die erste und einzige sprechende Instanz. Gunnar und Hrútur, deren Repliken in wörtlicher Rede zitiert werden, sind also keine aktiven Sprecher innerhalb dieser Szene. Während der Lektüre dieser Passage werden damit zwei Situationen in überlappender Form vermittelt und müssen entsprechend durch einen Leser erschlossen werden. Leser müssen dabei berücksichtigen, dass Hrútur zu diesem Zeitpunkt nicht anwesend ist. Gunnar, der hier zwar in wörtlicher Rede zitiert wird, ist allerdings (im Rahmen der fiktiven Szene) tatsächlich lediglich in der Position des stummen Zuhörers anwesend, während Njáll spricht. Da die Darstellungsform und Informationsvermittlung dieses Textabschnittes so gewählt und gestaltet ist, können Leser gegenwärtige und zukünftige Handlungen in einem Zuge kognitiv konstruieren und repräsentieren. Aus diesem Grund muss die eigentliche Umsetzung des Plans nicht noch einmal ausführlich geschildert werden, um dem Leser ein kohärentes Verständnis der Handlungsabfolge zu ermöglichen. Dieser Handlungsabschnitt ist daher im anschließenden Kapitel 23, das in der hier verwendeten Englischen Ausgabe die Kapitelüberschrift ,Gunnar Follows Njál's Advice ‘ trägt, lediglich überblicksartig zusammengefasst.

Um , die Weichen' für solche Verstehensprozesse zu schaffen, wird an dieser Stelle ein Szenario genutzt, in welchem die Figuren und Leser motivierende mentale Haltungen attribuieren müssen und zukünftiges Verhalten darauf basierend vorhersagen bzw. nachvollziehen können. Durch die gewählte Form der Vorhersage wird die kognitive Rekonstruktion zukünftiger Ereignisse seitens des Lesers in die Rekonstruktion gegenwärtiger Ereignisse eingebettet. Damit wird auf eine weitere Art und Weise durch die Beschaffenheit der Darstellungsform interpretative Komplexität geschaffen. Erneut - wenn auch in anderer Form bildet die Funktionalität der ToM des Lesers den Rahmen dafür, eine Interpretationssituation zu schaffen, die über die reine Rekonstruktion einer schlichten Ereignisabfolge hinausgeht. 
Ich möchte an dieser Stelle ein kurzes Fazit ziehen, bevor ich mich einem zweiten Text im Vergleich zuwende: In den vergangenen Abschnitten ist deutlich geworden, dass die Attribution motivierender mentaler Haltungen durch einen Leser ein wichtiges und durch die Informationssituation des Textes gelenktes Element der kognitiven Rezeption der Njáls Saga darstellt. Ohne die zusätzliche und eigenständige Attributionsleistung des Lesers ist eine kohärente Interpretation in diesem Fall nicht möglich. Ich habe ebenfalls einige Merkmale (z. B. die Erzählsituation und Informationsordnung) des Textes beschrieben und geprüft inwiefern sie die Interpretationsprozesse eines Lesers in diesem spezifischen Fall lenken. Drittens habe ich zwei ausgewählte Passagen vorgestellt, die durch ihre besondere Strukturierung und in Verbindung mit der Funktionalität der ToM des Lesers komplexe Interpretationssituationen schaffen. Hier werden entweder besondere kognitive Leistungen seitens des Lesers ermöglicht (z. B. im Fall Eyjólfur die Eskalation der Motivationsattribution von der Ebene der Figurenmotivierung auf die Ebene der Motivierung der Textgestaltung) oder erzwingen (z. B. die gekoppelte Rekonstruktion zweier zeitlich aufeinanderfolgender Szenen).

Aus methodischer Sicht lässt sich festhalten, dass die semantische und strukturelle Beschaffenheit der Njáls Saga eine Reihe von Anhaltspunkten bietet, die es gestatten, Rückschlüsse auf mögliche Rezeptions- und Interpretationsprozesse ihrer Leser zu ziehen. Es lässt sich an Hand der Analyse von einzelnen Passagen und ihrem Kontext ein Strukturprofil des Textes erstellen an Hand dessen einem Modell-Leser Interpretationsprozesse zugeschrieben werden können. Dabei stehen nicht nur ToM-relevante Interaktionen auf (fiktiver) Handlungsebene im Fokus. Es geht vielmehr auch darum, die Rolle der ToM-relevanten kognitiven Mechanismen genauer zu beleuchten. Darüber hinaus wird das Prinzip der ToM an dieser Stelle als textanalytische Methode übernommen, indem einem Modell-Leser basierend auf einer Reihe von Informationen (dem Strukturprofil des Textes und dem Wissen um die Funktion bestimmter kognitiver Mechanismen) Rezeptionsprozesse attribuiert werden.

Aufbauend auf die hier zusammengetragenen Überlegungen zur Njáls Saga wäre es im Rahmen weiterführender Studien möglich, sich literarhistorischen- oder gattungsspezifischen Fragestellungen zuzuwenden und zu überprüfen, ob sich parallele Rezeptionsbedingungen in anderen Werken derselben Gattung oder desselben Entstehungszeitraums identifizieren lassen. Das sei zukünftigen Projekten vorbehalten. Gleichzeitig ist aber auch ein Vergleich mit anderen narrativen Texten hinsichtlich ihrer kognitiven Leistungsanforderungen möglich. Ich wähle hierfür das Erzählstück Gehen von Thomas Bernhard, welches einer anderen 
Textgattung als die Njáls Saga angehört, deutlich weniger umfangreich ist und in einer zeitlich weit entfernten Epoche (dem 20. Jahrhundert) entstanden ist. Ich möchte zeigen, dass sich mittels der gewählten Analysemethode auch im Hinblick auf diesen deutlich andersartigen Text Erkenntnisse über die Rezeptionsbedingungen, die er an seine Leser stellt, gewinnen lassen und ein Vergleich aus kognitivistischer Hinsicht daher möglich ist. Es stellt sich die Frage, ob sich herausfinden lässt, dass die Unterschiede in der Beschaffenheit der Texte tatsächlich deutlich voneinander abweichende kognitiven Interpretationsvorgänge erfordern oder ob sich trotz der Unterschiede Gemeinsamkeiten in der kognitiven Rezeption identifizieren lassen. 


\section{Gehen}

\subsection{Einführung - Inhalt und bestehende Forschungsschwerpunkte}

Worum geht es in Gehen? In Bernhards kurzem Erzählstück werden die Gespräche von verschiedenen Figurenpaaren dargestellt, die in verschiedenen Konstellationen zu verschiedenen Zeitpunkten miteinander spazieren gehen. Im Zuge dieser Gespräche wird gleichzeitig eine kurze, zentrale Ereignisfolge rekonstruiert. Genauer gesagt: Gegenstand des Erzählstückes Gehen (1971) ${ }^{63}$ sind drei verschiedene Gesprächssituationen, an denen insgesamt fünf fiktive Personen (Oehler, Karrer, Scherrer, der Textilverkäufer Rustenschacher und der namenlose Ich-Erzähler) beteiligt sind. Darüber hinaus sind zwei weitere fiktive Charaktere (Hollensteiner und dessen Schwester) Gegenstand der einzelnen Gespräche, ohne während der dargestellten Handlungen , anwesend` zu sein. Im Rahmen der evozierten Unterhaltungen entsteht Raum für Diskurse über sprachphilosophische Themen, die mit intertextuellen Verweisen (u. a. auf die Arbeiten Ludwig Wittgensteins und Ferdinand Ebners) einhergehen. Im Verlauf der verschiedenen Unterhaltungen wird ebenfalls eine kurze Ereignisfolge (die Begegnung im rustenschacherschen Laden und Karrers anschließendes Verrücktwerden) rekapituliert. Die gewählte Erzählform und Darstellung der Gespräche soll an späterer Stelle genauer beschrieben werden soll. Das erste, ,äußere' Gespräch zwischen dem Ich-Erzähler und Oehler bildet den Rahmen für die rückblickende Rekonstruktion von zwei weiteren Unterhaltungen aus der Vergangenheit. An der zweiten (im Verlauf des ,Rahmen`- Gespräches rekonstruierten) zeitlich zurückliegenden Unterhaltung nehmen hauptsächlich Karrer und Oehler, später auch für kurze Zeit der Textilverkäufer Rustenschacher, teil. Im Anschluss an eine Konfrontation mit dem Textilverkäufer, der laut Karrer anstatt erstklassiger englischer Stoffe tschechoslowakische Ausschussware mit ,merkwürdigen, schütteren Stellen` verkauft, verfällt Karrer dem Wahnsinn. Er wird anschließend in eine psychiatrische Heilanstalt, weshalb er an den gewohnten Spaziergängen mit Oehler nicht mehr teilnehmen kann. Der namenlose Erzähler übernimmt infolgedessen die vakante Rolle des Spaziergängers an Oehlers Seite. So entsteht der Rahmen für die (zeitlich gesprochen) gegenwärtige (Rahmen) Unterhaltung zwischen dem Ich-Erzähler und Oehler, in die die Schilderung aller weiteren

\footnotetext{
${ }^{63}$ Die für diese Arbeit verwendete Ausgabe ist die folgende: Bernhard, T. (2013). Gehen. Frankfurt am Main: Suhrkamp (nachfolgend zitiert als, $\mathrm{GN}^{\circ}$ ).
} 
Gespräche eingebettet ist. Die dritte, ebenfalls eingebettete Unterhaltung, von der im Rahmen des ,Rahmen“- Gespräches zwischen dem Erzähler und Oehler die Rede ist, findet zwischen Oehler und Scherrer, Karrers behandelndem Psychiater, im Anschluss an dessen Einlieferung statt. Der Anlass ist die Suche nach einer möglichen Ursache für Karrers Verrücktwerden. Oehler begründet dies in diesem Kontext u. a. mit Karrers extensiven und ergebnislosen Grübeleien über das Denken selbst (vgl. GN, S. 73 f.).

Die Menge der Forschungsliteratur, die sich mit Thomas Bernhards Kurzprosastück Gehen beschäftigt, ist - womöglich aufgrund des jungen Alters und geringen Umfangs des Werkes - vergleichsweise klein. Dennoch möchte ich (wie auch schon im Fall der Njáls Saga) die Ansätze und Hypothesen der vorliegenden Arbeiten als Anhaltspunkt für auffällige und charakteristische Textmerkmale nutzen. Einen wichtigen Beitrag leistete Claudia Albes (1999) mit ihrer Dissertationsschrift Der Spaziergang als Erzählmodell. Studien zu Jean-Jacques Rousseau, Adalbert Stifter, Robert Walser und Thomas Bernhard. Ihre Untersuchungen gelten der Verbindung zwischen Form und Inhalt der Narration mit dem Ziel, ,[...] die immer noch weitgehend dunkle Beziehung zwischen literarischen Aussageprozessen (dem Erzählen) und dem literarisch Ausgesagten (der Erzählung)“(Albes, 1999, S. 12) zu erhellen. Albes untersucht in diesem Zusammenhang Texte, die vom Spazierengehen handeln und zugleich, mit ihren Worten, ,spaziergängerisch erzählt' sind (ebd.). Ihre Arbeit folgt der Frage, inwiefern literarische Erzählverfahren durch die Merkmale einer Tätigkeit - nämlich die des Spazierengehens - modelliert werden. Mit Thomas Bernhards Gehen analysiert sie einen Text, in dem der Spaziergang zugleich ein zentrales Motiv der Handlung ist. Im Vergleich mit anderen Forschungsbeiträgen, die sich mit diesem Motiv beschäftigen, folgt ihre zweiteilige Textanalyse hauptsächlich der Frage nach dem ästhetischen Prinzip, das Form und Inhalt des Werkes verbindet. Albes betont, dass Bernhards Text einen besonders markanten Meilenstein in der Geschichte der ,Spaziergänger'-Texte bildet, da er ,,[...] die in diesen Texten angelegten poetologischen Möglichkeiten bündelt und reflektiert““ (ebd. S. 312). Gehen thematisiert die Schwierigkeit, Sinn sprachlich zu vermitteln, nicht nur inhaltlich, sondern bildet sie gleichzeitig auf struktureller Ebene mit der Wahl der narrativen Form und den zahlreichen repetitiven sprachlichen Elementen und Mustern ab. Albes Beobachtungen bezüglich der narrativen Form und der besonderen sprachlichen Struktur des Werkes bilden eine wichtige Grundlage für die Analysen im Kontext der hier relevanten Forschungsfrage, da hier bereits eine Reihe auffälliger struktureller und ästhetischer Merkmale des Textes beschrieben werden. Die von ihr geleisteten Analysen dieser Merkmale stehen allerdings im Fokus einer ästhetischen Fragestellung, so dass sie hier - im Rahmen einer kognitivistisch 
orientierten Arbeit - lediglich als Quelle für Hinweise auf die wesentlichen strukturellen und inhaltlichen Merkmale des Textes dienlich sind.

Auch Jonathan James Long (2001, S. 3) stellt im Rahmen seiner Monographie The novels of Thomas Bernhard. Form and its function die Frage, inwiefern die Werke Thomas Bernhards das Problem der narrativen Vermittlung von Inhalten selbst thematisieren. Seine These lautet, dass Bernhards Werke die Referenzproblematik von sprachlichen Zeichen nicht nur inhaltlich erwähnen, sondern auch auf formaler Ebene spiegeln. Long beschreibt Bernhards Poetik als ein Konzept der ,Analogie‘, wobei sich der Referenzcharakter sprachlicher Zeichen auf der Darstellungsebene verschiedener Werke dieses Autors spiegelt. Das dadurch aufgeworfene Problem des besonderen Zusammenhangs von Sprache und Denken ist - als eine Konsequenz davon - ein häufiges Thema in Bernhards Werken (ebd.). Zu dem Werk Gehen selbst finden sich in Longs Monographie nur wenige Ausführungen. Hilfreich im gegenwärtigen Kontext ist allerdings sein Hinweis auf die eng verknüpfte Thematisierung und strukturelle Reflexion der Referenzproblematik sprachlicher Zeichen. So wechselt die Referenz feststehender sprachlicher Zeichen (insbesondere von Personalpronomina) in Gehen an vielen Stellen. An anderen Stellen wird wie gebrochen oder gar aufgehoben. ${ }^{64}$ Das wirft die an späterer Stelle zu klärende Frage auf, ob und inwiefern dies die online Rezeption des Werkes und die kognitive Verarbeitung der gegebenen Informationen während der Lektüre beeinflusst.

Michael Billenkamp (2008) legte mit seiner Monographie Thomas Bernhard. Narrativik und poetologische Praxis eine umfassende Analyse des Gesamtwerkes des Autors vor. Er verfolgt in dieser Arbeit das Ziel, das Werk Thomas Bernhards mit Hilfe eines hermeneutischen Ansatzes zu klassifizieren und den individuellen Werdegang des Autors als Künstler nachzuzeichnen. Die im Rahmen seiner Analysen gewonnenen Erkenntnisse nutzt Billenkamp zur Erarbeitung eines poetologischen Gesamtprogramms, das dem Autor zugeschrieben wird, wobei er die wichtigsten Themenkomplexe sowie formelle und konzeptionelle Aspekte von Bernhards einzelnen Werken zusammenstellt (Billenkamp, 2008, S. 18). Vor allem Billenkamps Überlegungen zur Ich-Konstruktion der für das Frühwerk Bernhards typischen anonymen Erzählinstanz und zum Kunstcharakter der verwendeten Sprache sind im Hinblick auf die in dieser Arbeit gestellten Fragen relevant. Es stellt sich auch hier die Frage,

\footnotetext{
${ }^{64}$ Beispielhafte Textpassagen, in denen sich dies wiederfindet nenne ich an relevanter Stelle im Zuge der Analyse.
} 
wie sich die Strategie der Konfiguration und Konstruktion der Erzählinstanz auf die kognitive Rekonstruktion durch einen Leser auswirkt. Billenkamp unterstreicht die Beobachtung, dass das berichtende ,Ich` im gesamten Werk Bernhards häufig nur den Vermittlungspart zwischen Lesern und den Protagonisten der Texte übernimmt. Herkunft, Identität und Status dieses ,Ichs` bleiben dabei zumeist im Dunkeln und die Funktion der Erzählinstanz beschränkt sich ihm zufolge häufig lediglich auf das kommentarlose Protokollieren der Sätze eines Gegenübers (ebd. S. 190). Billenkamps Schlussfolgerung hinsichtlich einer möglichen Wirkung dieser narrativen Strategien auf den Leser, der sich mit einer solchen Erzählinstanz konfrontiert sieht, lautet:

Auf diese Weise fördert Bernhard nicht nur die Distanzierung des Lesers gegenüber dem Erzählten, sondern unterbindet auch die Möglichkeit einer identifikatorischen Rezeption. [...] Erschwert wird dies dadurch, dass der Rezipient auf die subjektiven Eindrücke und Wahrnehmungen der Protokollanten angewiesen ist, die den Texten einen spekulativen Gestus verleihen. Sich von einzelnen Figuren oder Geschehnissen ein neutrales oder objektives Bild zu machen, erscheint insofern unmöglich. (Ebd.)

Aus der Sicht einer kognitivistischen Untersuchung der Wirkung bestimmter Textstrukturen ist Billenkamps Schlussfolgerung eine Hypothese, die im Rahmen einer entsprechend ausgerichteten (theoretischen oder empirischen) Arbeit überprüft werden müsste. Ich möchte seine Hinweise zum Anlass nehmen auch die generelle Konstruktion und Struktur der Erzählsituation von Bernhards Erzählstück im Zuge meiner nachfolgenden Überlegungen zum Zusammenhang der Textmerkmale und der kognitiven Rezeption des Werkes zu berücksichtigen.

Abschließend möchte ich auch Manfred Jurgensens Arbeit nennen, der sich mit Die Sprachpartituren des Thomas Bernhard einem weiteren auffälligen Charakteristikum - nämlich den besonderen sprachkünstlerischen Elementen - des Werkes zuwendet (Jürgensen, 1981). Die im Rahmen seiner Arbeit zusammengetragenen Beobachtungen möchte ich nicht hier zusammenfassend, sondern erst an relevanter Stelle im Einzelnen nennen. Sie dienen mir an späterer Stelle als Hinweise auf die Beschaffenheit und Struktur der Informationen, mit denen ein Leser während der Lektüre des Werkes konfrontiert ist und damit als wichtige Indizien zur Beschreibung der kognitiven Verstehensprozesse. Ich möchte hier lediglich abschließend festhalten, dass die wichtigsten, in der Forschung zum Werk Gehen diskutierten Merkmale des Textes die poetische Selbstreflexivität, die Sprachästhetik und die besondere Erzählsituation bzw. Konstitution der Erzählinstanz sind. Diese Merkmale sollen im Verlauf 
der nachfolgenden Untersuchungen besondere Beachtung finden. Darüber hinaus gilt es wie auch im Hinblick auf die Njáls Saga - das Informationssystem des Textes genauer zu betrachten, um die grundlegenden Voraussetzungen für die kognitive Rezeption dieses Werkes genauer bestimmen zu können.

\subsection{Form: die Erzählsituation in Gehen}

Der Versuch, die erzählerische Situation in Gehen mit den zur Verfügung stehenden Begriffen der Narratologie zu erfassen, ist auf Grund der komplexen Schachtelung von Sprechinstanzen, die dieser Text evoziert, schwierig. Dennoch möchte ich im Sinne der hier gestellten Forschungsfragen zur kognitiven Rezeption, aber auch im Hinblick auf einen Vergleich mit dem zuvor analysierten Werk, die durch die Erzählsituation geschaffenen Rezeptionsbedingungen dieses Textes genauer betrachten. Anders als in der Njáls Saga wird in Gehen lediglich eine kurze Ereigniskette mit Hilfe einer werkübergreifenden, geschachtelten narrativen Strategie geschildert. Bereits die einleitenden Sätze des Textes legen diese komplexe Erzählstrategie des Zitates direkter Redeinhalte einer Figur durch eine andere Figur und in äußerster Instanz durch den homodiegetischen Ich-Erzähler offen. Sie lauten:

Während ich, bevor Karrer verrückt geworden ist, nur am Mittwoch mit Oehler gegangen bin, gehe ich jetzt, nachdem Karrer verrückt geworden ist, auch am Montag mit Oehler. Weil Karrer am Montag mit mir gegangen ist, gehen Sie, nachdem Karrer am Montag nicht mehr mit mir geht, auch am Montag mit mir, sagt Oehler, nachdem Karrer verrückt und sofort nach Steinhof hinaufgekommen ist. (GN, S. 7). ${ }^{65}$

Dank der deiktischen Informationen, die bereits in diesen ersten Sätzen des Werkes vermittelt werden, (,Während ich, bevor Karrer verrückt geworden ist, nur am Mittwoch mit Oehler gegangen bin, gehe ich jetzt, nachdem Karrer verrückt geworden ist, auch am Montag mit Oehler'), zeigen einem Leser an, dass es innerhalb der durch den Text evozierten Fiktionswelt einen Zeitraum vor und einen Zeitraum nach Karrers Verrücktwerden gibt. Der Moment des Zusammenbruchs der genannten Figur stellt einen Wendepunkt dar, der dazu führt, dass nicht mehr Oehler und Karrer, sondern der namenlose Ich-Erzähler und Oehler montags gemeinsam spazieren gehen. Der Redeinhalt dieses Satzes ist dem Erzähler zuzuschreiben. Der anschließende zweite Satz des Textes enthält dieselben semantischen Informationen wie

\footnotetext{
${ }^{65}$ Meine Hervorhebungen.
} 
der erste. Es handelt sich jedoch um das Zitat eines Redeinhaltes, das Oehler zuzuschreiben ist - dieselben Ereignis-Information (Oehler geht nun montags mit dem Erzähler anstatt mit Karrer spazieren) wird hier aus dem Blickwinkel und mit den Worten Oehlers präsentiert. Der Einschub ,sagt Oehler' kennzeichnet diese Passage als Zitat. Der Perspektivwechsel geht mit der kognitiven Aufgabe einher, einen Teil der deiktischen Informationen - in diesem Fall die Personalpronomen - umzudeuten. Der erste der beiden zitierten Sätze evoziert durch die Verwendung des Personalpronomens ,ich“ und die Nennung eines Namens (,Oehler') zwei deiktische Bezugspunkte: diejenige eines Sprechers und diejenige einer Person, über die gesprochen wird. Die Sprecherrolle im ersten Satz liegt bei der namenlosen Erzählinstanz. Nach den gewohnten grammatikalischen Regeln der Anaphorik, müsste sich das im zweiten Satz folgende Personalpronomen ,mir` auf die Erzählfigur beziehen. Auch die Anrede einer Person mit ,Sie“ müsste, der deiktischen Vorgabe des ersten Satzes entsprechend, an jemand anderen als die Erzählfigur selbst gerichtet sein. Beides ist aufgrund der Verlagerung der Perspektive jedoch nicht der Fall. Durch die Erzählstrategie des Zitates von direkten Redeinhalten in Gehen, die sich durch das gesamte Werk zieht, ist das ,Ich“ des Textes wie in diesem Fall - wie auch an vielen anderen Stellen - nicht dem Ich-Erzähler vorbehalten. Auch die Bezugspunkte anderer deiktischer Zeichnen wechseln an vielen Stellen von einem Satz zum anderen. Sie müssen daher häufig durch den Leser, in Abhängigkeit von der gegenwärtig gültigen Perspektive des Geschilderten, unterschiedlichen Sprechern zugeordnet werden.

Der Schwierigkeitsgrad solcher interpretativen Prozesse wird im Verlauf der Lektüre gesteigert, indem Redeinhalte weiterer Sprecherinstanzen in geschachtelter Form zusätzlich in die Sätze eingefügt sind. Ein Beispiel hierfür ist der folgende Satz: „Denn, so Karrer, sagt Oehler, die Möglichkeit, daß Hollensteiner aus innerer Ursache Selbstmord machen könne, habe immer bestanden [...]“ (GN, S. 47). Der Inhalt dieser Aussage lautet, dass immer die Möglichkeit bestanden habe, dass Hollensteiner aus innerer Ursache Selbstmord begehen könne. Dieser Gedanke wird ursprünglich durch Karrer geäußert, der zunächst durch Oehler, der wiederum durch den Ich-Erzähler zitiert wird. Alle diese Schritte sind durch entsprechende Zuweisungsformen (,so Karrer', , sagt Oehler') eindeutig gekennzeichnet, so dass eine Zuordnung der Redeinhalte und eine Rückentschlüsselung der Sprecherkonstellation für den Rezipienten möglich ist. Der Erzähler (1. Stufe) vermittelt hier an den Leser, dass Oehler sagt (2. Stufe), dass Karrer gesagt hat (3. Stufe), dass etwas der Fall ist. Schachtelstrukturen wie diese zu verstehen, die durch die Staffelung mehrerer Ebenen von Komplementsatzstrukturen erreicht wird, erfordert besondere kognitiven Leistungen, die auch für die ToM 
von zentraler Bedeutung sind. Um noch einmal zu rekapitulieren: Im Zuge der Forschung zur Korrelation zwischen dem Verständnis von falschen Überzeugungen und mehrfach verketteter mentaler Haltungen (z. B. A denkt, dass B denkt, dass C der Überzeugung ist, dass $\mathrm{X}$ der Fall ist) und der Entwicklung sprachlicher Fähigkeiten wurde eine starke und zuverlässige Korrelation zwischen den ToM-Fähigkeiten und der Entwicklung syntaktischer Kompetenzen festgestellt. Eine solche Korrelation lässt sich einerseits durch die Fähigkeit erklären, in beiden Fällen die unterschiedlichen funktionalen Elemente der oben als Beispiel aufgeführten Verstehenssituation (oder eines Satzes) semantisch trennen und mit unterschiedlichen Wahrheitswerten versehen zu können. Während es wahr ist, dass A denkt, dass B denkt, dass C der Überzeugung ist, dass X der Fall ist, ist es womöglich nicht wahr, dass $\mathrm{X}$ der Fall ist. Gleichzeitig müssen die Inhalte der einzelnen Satzelemente kognitiv einer denkenden/sprechenden Instanz zugeordnet werden. So wie ein Mensch im Zuge der ToMAufgabe zurückverfolgen muss, wer etwas denkt und was ggf. tatsächlich der Fall ist, so muss ein Leser von Thomas Bernhards Erzählstück in vielen Fällen kognitiv rekonstruieren, wer etwas sagt, wer sagt, dass ein anderer etwas gesagt hat, und was tatsächlich geschehen ist.

Es finden sich weitere Passagen, in denen die Schachtelung der Ebenen der Berichterstattungen - zumindest auf den ersten Blick noch - zusätzlich erhöht wird, indem nicht nur Sprecher, sondern auch die jeweils relevanten Adressaten aufgezählt sind. Folgende Passage sei beispielhaft für einen Extremwert zitiert, den die Verzahnung des Sprecher- und Adressatensystems innerhalb von Gehen erreichen kann:

Plötzlich sagt Karrer zu Rustenschacher, so Oehler zu Scherrer, wenn Sie, Rustenschacher, genau hinter dieser Hose, die mir gerade von Ihrem Neffen gegen das Licht gehalten wird, Aufstellung nehmen, genau hinter dieser mir von Ihrem Neffen hinter das Licht gehaltenen Hose, kann ich durch diese Hose Ihr Gesicht mit einer Deutlichkeit sehen, mit welcher ich Ihr Gesicht gar nicht sehen will. (GN, S. 62).

Hier spricht auf innerster Ebene Karrer (K) mit Rustenschacher (R). Es sind die Redeinhalte von Karrer, die innerhalb dieser Textstelle vermittelt werden. Auf einer zweiten Gesprächsebene gibt Oehler (O) die Redeinhalte Karrers, Scherrer (S) gegenüber wieder. Auf einer dritten Gesprächsebene berichtet Oehler dem Ich-Erzähler (E) von diesem Gespräch mit Scherrer über das Gespräch Karrers mit Rustenschacher. Auf der äußeren Kommunikationsebene, auch wenn diese hier nicht deutlich markiert ist, vermittelt der Ich-Erzähler die gesamte dargestellte Gesprächskonstellation zusammen mit den Redeinhalten der einzelnen 
Sprecherinstanzen. Die Benennung der einzelnen Sprecherinstanzen mit ihren Namen erfolgt expositorisch zu Beginn der Textpassage, woraufhin im weiteren Satz ausschließlich deklinierte Personalpronomen folgen, die der Rezipient den einzelnen Ebenen und dem jeweiligen deiktischen Zentrum zuordnen muss. Nach einer längeren Fortführung der gestaffelten Berichterstattung über die Unterhaltung zwischen Karrer und Rustenschacher schließt der Abschnitt mit einer Rückkehr zu den eigentlichen Namen der beteiligten Personen in reziproker Reihenfolge:

[...] Und wie lange suche er schon den rustenschacherschen Laden auf und wieviele Hosen habe er schon im rustenschacherschien Laden gekauft, sagt Karrer, so Oehler zu Scherrer. Kein einziger abgerissener Knopf! Sagt Karrer, so Oehler zu Scherrer, keine einzige aufgerissene Naht! Sagt Karrer zu Rustenschacher. (GN, S. 63).

Die Formel ,sagt Karrer zu Rustenschacher', welche die hier besprochene Passage einleitet, steht auch an ihrem Ende. Die Redeinhalte Karrers selbst wurden im Verlauf der Textstelle von den Formeln ,so Oehler zu Scherrer' gerahmt, um die hier errichtete Staffelung der Gesprächssituationen und der beteiligten Sprecherinstanzen zu verdeutlichen. Diese Staffelung besteht aus insgesamt vier direkt markierten Sprechern (Karrer, Rustenschacher, Oehler und Scherrer) und einem indirekt assoziierten deiktischen Bezugspunkt - dem Ich-Erzähler. Die Besonderheit dieser und aller vergleichbaren Textpassagen besteht in der Konstruktion einer Interpretationssituation, in der eine kognitive Leistung des Rezipienten abgefragt wird, die der komplexen Schachtelung der Sprecherkonstellation gerecht werden muss. Um diese Passage verstehen zu können, müssen Leser die Vielzahl sprechender Instanzen, Adressaten und den Gesprächsinhalt selbst (E sagt, dass O sagt, dass $\mathrm{O}$ zu S sagte, dass K zu R gesagt hat) ,entwirren'. Es muss dabei gleichzeitig gelingen, relevante Informationen über die durch den Text vermittelten Situationen (z. B. im rustenschacherschen Laden oder während des Spazierganges) zu rekonstruieren. Im Fall von Gehen werden in der beschriebenen geschachtelten Form tatsächlich unterschiedliche Handlungssituationen geschildert. Wenn Leser korrekt rekonstruieren wollen, wer zu welchem Zeitpunkt etwas zu wem gesagt hat (und darauf aufbauend, welche Ereignisse sich wann innerhalb der Fiktionswelt abspielen), dann müssen sie nicht nur Sprecherinstanzen die richtigen Redeinhalte, sondern auch die jeweils passenden Spaziergängerpartner oder ,Zuhörer` zuordnen. Das würde für eine Erleichterung der kognitiven Anforderungen sprechen, wenn Adressaten und Sprecher explizit benannt sind. Allerdings wird die grammatische Struktur eines Satzes, in welchem Sprecher und Adressaten benannt sind, komplexer und es ließe sich auch spekulieren, dass Lesern in diesem Fall 
vor anspruchsvollere Aufgaben gestellt werden, wenn sie verstehensrelevante Satzelemente kognitiv filtern

und (um-) sortieren müssen.

Allerdings lässt sich an dieser Stelle festhalten, dass die Struktur der Darstellungsform in Gehen aufgrund der strukturellen Parallelen zwischen den kognitiven Leistungsanforderungen einen wichtigen kognitiven Mechanismus der ToM anspricht, der für die Interpretation der vermittelten Informationen notwendig ist. Diese Hypothese möchte ich deshalb unterstreichen, weil in diesem Text die grammatische Struktur des Komplementsatzes als dominanten Darstellungsprinzip zu beschreiben ist. Die zitierten und beschriebenen Textpassagen wurden hier als Beispiele für die werkübergreifende Erzählstrategie zitiert. Die relevanten kognitiven Mechanismen werden dadurch aktiviert, dass durch eine bestimmte Gestaltung der Satzgrammatik - aus struktureller Sicht - ähnliche kognitive Verstehensaufgaben geschaffen werden, wie in den Fällen, in denen Menschen mehrfach, geschachtelte Intentionsoder Überzeugungssituationen verstehen müssen. Auch hier liegt die entscheidende Fähigkeit in der Rekonstruktion einer Situation über die korrekte Zuordnung von Personen und den Inhalten und Funktionen ihrer mentalen Haltungen. Im Fall von Gehen handelt es sich nicht um die Darstellung einer Verkettung mentaler Haltungen, sondern um die Verkettung und Verschachtelung von Gesprächsinhalten. Die strukturelle Parallele beider Interpretationsvorgänge besteht darin, dass in beiden Fällen semantische Informationen über eine bestimmte Situation in Relation zu den funktionalen Eigenschaften der Informationsvermittlung ausgewertet werden müssen.

\subsection{Kognitive Rekonstruktion der fiktiven Handlung}

Die Ereignisfolge und Figurenkonstellation, die in Gehen geschildert wird und an früherer Stelle bereits umrissen wurde, ist wesentlich weniger umfangreich, als die der Njáls Saga. Ich habe die einzelnen Figuren, Gesprächssituationen und die kurze Handlungsfolge bereits zuvor kurz umrissen. Trotz dieser recht einfach anmutenden Struktur des dargestellten Geschehens ist die Aufgabe der kognitiven Rekonstruktion der Ereignisse aufgrund der zuvor beschriebenen komplexen Vermittlungssituation nicht trivial. Um ein Verständnis des Geschehens zu gewährleisten, ist es notwendig, dass ein Leser in der Lage ist, alle einzelnen Ereignisfolgen während der Lektüre in angemessener Form in das Modell der erzählten Situation einzubinden. Er muss zu jedem Zeitpunkt eine Zuordnung zwischen den durch den 
Text vermittelten Informationen und den Ereignisabläufen innerhalb der geschilderten Situationen leisten. Ich habe im vergangenen Unterkapitel beschrieben, inwiefern die ,Schachtelung' in der Darstellungsform der einzelnen Gespräche eine besondere Kognitionsleistung erfordert. Wenn ein Leser diesen besonderen Text zur Hand nimmt und zu lesen beginnt, muss er fortlaufend nachvollziehen, wer was über wen in welcher Situation gesagt hat, um anschließend rekonstruieren zu können, welche Situationen in welcher Form stattgefunden haben. Die Ordnung der Informationsvergabe spielt dabei eine besondere Rolle, da sie darüber bestimmt, welche Informationen Leser zu welchem Zeitpunkt der Lektüre erhalten und dementsprechend zu welchem Zeitpunkt diese Informationen in die bereits erstellte kognitive Repräsentation des Erzählten integriert werden können bzw. müssen. Aufgrund der bereits beschriebenen, geschachtelten Gesprächssituation müssen Leser während der fortlaufenden Lektüre des Textes durchgehend zeitdeckend mehrere Handlungsverläufe und Gesprächssituationen rekonstruieren. Ich habe bereits einführend kurz beschrieben, dass der erste Handlungsbogen den Spaziergang des Ich-Erzählers mit Oehler und das in diesem Kontext geführte Gespräch umspannt. Der erste Satz des Werkes besteht in einem Sprechakt des Erzählers. Im Diskurs der Erzähltheorie wurde der Adressat eines solchen allgemeinen narrativen Sprechaktes z. B. als ,narratee‘ (z. B. Prince, 1985) bezeichnet. Gemeinsam bilden diese beiden Instanzen die äußere Erzählebene (Ebene 1) der Informationsvermittlung. Wenn Leser beim zweiten Satz des Textes angelangt sind, wird deutlich, dass sich eine weitere Sprecher-/Adressaten Ebene eröffnet. Offenbar sprechen der Erzähler und Oehler im Kontext ihres neuerlichen gemeinsamen Spazierganges miteinander (Ebene 2). Im Zuge dessen werden weitere Handlungen, Situationen und Gespräche zwischen Karrer, und Oehler (Ereignis 1; Ebene 3) und zwischen Oehler und Scherrer (S) (Ereignis 2; Ebene 3) rekonstruiert. Oehler ist an allen Situationen der Ebene 3 sowie dem Gespräch der Ebene 2 beteiligt, so dass für einen Leser die Notwendigkeit entsteht, u. a. nachzuvollziehen welcher Ebene die wiedergegebenen Redeinhalte dieser Figur zuzuordnen sind. Ein Beispiel soll das veranschaulichen. In diesem Kontext ist Scherrer der eigentliche Gesprächspartner, dem Oehler von Karrers Verhalten berichtet. Oehler rekonstruiert im Zuge dessen ein weiteres Gespräch zwischen Karrer und Rustenschacher - einer Situation, an der er als Beobachter teilnimmt:

Der gleichen Ansicht war Karrer, sagt Oehler. Immer wieder sagt Oehler, der gleichen Ansicht war Karrer oder Karrer war ähnlicher Ansicht oder Karrer war anderer oder Karrer ist der entgegengesetzten Ansicht (oder Meinung) gewesen. Karrers Satz lautet immer wieder, sagt Oehler: [Wie sich die Menschen, die nicht wissen, wie sie dazu kommen, und die in 
keiner sie betreffenden Frage gefragt worden sind, und vor allem in den grundsätzlichsten Fragen nicht gefragt worden sind wie sich alle diese Menschen, mit welchen wir uns, wenn wir denken, immer und immer wieder mit der größten Zurechnungsfähigkeit identifizieren müssen, durch ihr ganzes Leben, gleich wer sie sind und gleich was sie sind und gleich wo sie sind, mit allen verabscheuungswürdigen Mitteln, also mit den Menschenmitteln, in ihr sie mit immer beängstigenderer Geschwindigkeit sich vervollkommnendes, endgültiges Unglück hinein- und hinauf- und hinuntertraktieren.] (GN, S. 21$)^{66}$

Die Schwierigkeit der hier zitierten Passage besteht darin, dass der der oben von mir in eckige Klammern gesetzte, mimetisch wiedergegebene Redeinhalt im Sinne einer kohärenten Interpretation Karrer zugesprochen werden muss. Dies ist anhand der gegebenen semantischen und strukturellen (grammatikalischen) Informationen im Text möglich, obwohl die letzte Inquit-Formel vor der entsprechenden Wiedergabe des Redeinhaltes , sagt Oehler' lautet. Auf der Repräsentationsebene des Rahmengesprächs hingegen stimmt die Zuordnung der Inquit-Formeln und entsprechenden Redeinhalte überein, da Oehler derjenige ist, der die Redeinhalte Karrers dem Ich-Erzähler gegenüber zitiert. Da hier verschiedene Gesprächssituationen ineinander gestaffelt vermittelt werden, müssen unterschiedliche deiktische (zeitliche, räumliche und personale) Referenzpunkte rekonstruiert werden, die der jeweiligen Situation zu eigen sind. Eine weitere Textpassage macht die Notwendigkeit hierfür noch deutlicher und ist selbst ohne kontextuelle Zuordnung verständlich:

Mit Karrer bin ich sehr oft hier gestanden unter dem Obenaus, sagt Oehler, und habe ihm von allen diesen fürchterlichen Zusammenhängen gesprochen. Dann sind wir, Oehler und ich, auf der Friedensbrücke. Es war bei dem Vorhaben Karrers, mir auf der Friedensbrücke einen wittgensteinschen Satz zu erklären, geblieben, aus Erschöpfung erwähnte Karrer nicht einmal mehr den Namen Wittgenstein auf der Friedensbrücke, ich selbst war zur Erwähnung des Namens Ferdinand Ebner nicht mehr fähig gewesen, so Oehler. (GN, S. 83)

Diese kurze Textpassage besteht aus drei Sätzen, wobei durch die Form des Zitats ein Perspektivwechsel zwischen dem Ich-Erzähler und Oehler suggeriert wird. Das hat zur Folge, dass Leser die sich wiederholenden Personalpronomina, die sich auf unterschiedliche Figuren beziehen, richtig zuzuordnen müssen, um darauf aufbauend die dargestellte fiktive Situation korrekt repräsentieren zu können. So bezieht sich das ,ich` des ersten Satzes auf Oehler, dessen Worte durch die Erzählfigur zitiert werden. Der semantische Gehalt des Satzes

\footnotetext{
${ }^{66}$ Klammern und Hervorhebung von mir.
} 
nimmt auf den gemeinsamen Spaziergang von Karrer und Oehler Bezug. Das erste Bezugswort des darauffolgenden Satzes ist das Personalpronomen , wir ${ }^{6}$, das sich jedoch, wie sich im weiteren Verlauf des Satzes herausstellt, nicht auf die beiden zuvor repräsentierten Gesprächspartner, sondern vielmehr auf den Ich-Erzähler und Oehler bezieht, die ihren gemeinsamen Spaziergang in der fiktiven Gegenwart unternehmen. Auf Grund dieser deiktischen Mikrostrukturierung wird die Verlagerung der narrativen Blickwinkel und der narrativen Zeit - und damit die Notwendigkeit zur kognitiven deiktischen ,Umorientierung` des Lesers veranlasst. Die Erzählstruktur erschwert die korrekte Zuordnung der Personalpronomina dadurch, dass der entscheidende Hinweis der den Perspektivwechsel markiert, nicht zu Beginn des Anschlusssatzes steht, sondern erst an einer nachgeordneten Stelle im Satz gegeben wird. Der Leser ist daher angehalten, die Bezüge der betroffenen Personalpronomina (z. B. diejenigen des ersten, wir') und die Anredeform, die er nach den ihm bekannten Regeln der Grammatik wahrscheinlich zunächst nicht korrekt zuordnet, ,rückwirkend' umzudeuten. Nachdem sich das ,ich' des ersten Satzes deshalb auf Oehler beziehen muss, da er in Form von indirekter Rede zitiert wird und demnach über sich selbst und seine früheren Erfahrungen mit Karrer berichtet, trägt das direkt im Anschluss, zu Beginn des Folgesatzes genannte ,wir` demnach zunächst eine trügerische Referenz auf die beiden Personen Karrer und Oehler. Die Verwendung der Präsensform (,sind`) ist der Schlüssel dafür, dass der Einschub ,Oehler und ich` sich nicht mehr auf O und K, sondern auf O und E bezieht. Die möglichen deiktischen Relationen gestatten an dieser Stelle nur diese eine Interpretationsmöglichkeit. In beiden Sätzen wird über Oehler in der dritten Person gesprochen. Der ausschlaggebende Unterschied besteht darin, dass der erste Satz die Wiedergabe von Redeinhalten in indirekter Form enthält, so dass zu jedem Zeitpunkt der Ich-Erzähler das Sprachrohr der vermittelten Informationen bleibt, die Blickrichtung auf die berichteten Ereignisse jedoch durch Oehler determiniert wird. Der Anschlusssatz hingegen provoziert eine Verlagerung der Perspektive, da das ,ich“ im deiktischen Koordinatensystem des Situationsmodells neu zugeordnet werden muss. Die Notwendigkeit zum Perspektivwechsel bringt neben dem personalen ebenfalls einen temporalen Shift in die Gegenwart des Geschehens mit sich. Eine lokale Verlagerung ist hingegen nicht notwendig, da sich beide Interaktionspaare sowohl in der fiktionalen Vergangenheit als auch in der fiktionalen Gegenwart an demselben Ort - auf der Friedensbrücke - befinden.

Der dritte Satz referiert im Anschluss die Redeanteile von Oehler, durch den Fokus der Erzählerfigur. Auch hier finden sich personale Referenzen (,ich ${ }^{\star}$ und , mir $^{\star}$ ), die allerdings in diesem Fall auf Oehler verweisen, so dass eine kognitive ,Rückverlagerung der kurzfristig 
umgestellten Perspektive durch den Rezipienten notwendig ist. Damit ist ein erneuter temporaler Shift notwendig, denn die inhaltlichen Informationen dieses Satzes beziehen sich auf die Ereignisse der fiktionalen Vergangenheit zum Zeitpunkt des Spazierganges von Oehler und Karrer. Die Konfiguration dieser speziellen Textpassage konfrontiert einen Leser mit der Aufgabe, zwei Ereignisse parallel zu rekonstruieren. An dem ersten Szenario, das hier kognitiv zu rekonstruieren ist, sind Karrer und Oehler beteiligt, die sich während ihres gemeinsamen Spazierganges auf Grund eines allgemeinen, Erschöpfungszustandes' nicht über Sätze von Wittgenstein und Ferdinand Ebner unterhalten können (ebd.). Das zweite Situation umfasst eine Unterhaltung über das vergangene Gespräch zu einem späteren Zeitpunkt, an der andere Figuren beteiligt sind. Die semantischen Informationen bezüglich des Gesprächsinhaltes zwischen Karrer und Oehler sind auf Grund der gewählten zitierenden Darstellungsweise der Situation in beide Repräsentationsstrukturen eingebunden.

Die Gestaltung der Informationsvermittlung erfordert an solchen Stellen die kognitive Rekonstruktion von zwei verschiedene Situationen, die zu verschiedenen Zeitpunkten innerhalb der Fiktionswelt (allerdings an demselben Ort) stattfinden und unterschiedliche Figurenkonstellationen betreffen. Weil innerhalb der Informationsstruktur der Passage abwechselnd auf die eine und auf die Situation referiert wird, sind Leser sind angehalten die zwei verschiedene Situationsmodelle nicht konsekutiv, sondern gleichzeitig zu rekonstruieren. Dies ist vor allem mit einem Blick zurück auf die zuvor vorgestellte Njáls Saga spannend. Die Erzählstrategien innerhalb der beiden Werke unterscheiden sich deutlich voneinander. Dennoch wird - mit unterschiedlichen Mitteln - ein vergleichbarer Effekt erzielt. Es finden sich in beiden Fällen Passagen, deren jeweilige narrative und semantische Beschaffenheit Strukturierung eine bestimmte kognitive Leistung von ihrem Leser erforderlich machen. Diese Leistung besteht in der gleichzeitigen Rekonstruktion zweier verschiedener Situationsmodelle. Auffällig ist, dass eine vergleichbare kognitive Leistung auch zum Leistungskatalog der ToM-Fähigkeit gehört. Wenn ein Mensch z. B. die falsche Überzeugung eines anderen über eine bestimmte Situation verstehen möchte, dann muss er zwei verschiedene Situationsmodelle erstellen, die er voneinander getrennt repräsentiert und als Repräsentation der tatsächlichen Situation vs. einer Repräsentation, die die falsche Überzeugung des Gegenübers wiedergibt, kognitiv kennzeichnet. Damit ist zu vermuten, dass hier aufgrund paralleler kognitiver Leistungsanforderungen möglicherweise dieselben kognitiven Mechanismen greifen. Wenn es stimmt, dass eine bestimmte Anzahl kognitiver Mechanismen verschiedene, aber strukturell miteinander vergleichbare funktionale Leistungen stützen, dann ist an dieser Stelle ein weiteres Beispiel dafür gefunden, dass die Mechanismen, die die ToM 
ermöglichen, in diesem Fall in einem anderen Kontext und durch einen differenten Stimulus aktiviert werden. Die Mechanismen der ToM können damit, allein aufgrund der strukturellen und semantischen Beschaffenheit eines Textes in die kognitive Rezeption literarischer Texte eingebunden werden, ohne, dass im Text auf inhaltlicher Ebene innere Vorgänge und mentale Haltungen dargestellt sind.

\subsection{Literarizitätsmerkmale und Metarepräsentation}

Es stellt sich abschließend die Frage, ob sich dieser Gedanke weiterführen und auf weitere Merkmale des Textes übertragenlässt. Ein Merkmal, dass in der Forschungsliteratur zu diesem Werk häufig angesprochen wird, ist der häufige Selbstbezug in der gewählten Darstellungsform. „Thomas Bernhards Erzählung ,Gehen“ erreicht eine Künstlichkeit der Form, wie sie weder in früheren noch in späteren Texten des Autors zu finden ist", schreibt Peter Kahrs (2000, S. 148) in seiner Monographie Thomas Bernhards frühe Erzählungen: rhetorische Lektüren aus dem Jahr 2000. Wenn die narrative Fortführung der Ereigniskette zugunsten von formal-ästhetischen Sprachspielen stillsteht, stellt sich die Frage, wie sich dies auf die bewusste und unbewusste kognitive Rezeption des Lesers auswirkt. Es ist denkbar, dass an solchen Stellen Raum für die Wahrnehmung formaler Aspekte geschaffen wird. In diesem Hinblick ist es denkbar, dass auf kognitiver Ebene die Bildung von Metarepräsentationen begünstigt wird - ein Mechanismus, der auch für den Leistungskatalog der ToMrelevant ist. Ohne empirische Mittel lässt sich diese Frage nicht endgültig beantworten. Dennoch hilft ein Blick auf die Beschaffenheit bestimmter Textstellen, um diese Hypothesen zu konkretisieren.

Claudia Albes (1999, S. 296) nennt die sprachliche Dichte des Werkes in diesem Kontext als eines seiner zentralen Merkmale. Sprachliche Dichte wird in diesem Werk vor allem durch das Stilmittel der Perseveration erreicht. Der Begriff der Perseveration (von lat. ,perseverare': beharren) wurde ursprünglich in der Psychopathologie für Störungen verwendet, die mit einer zwanghaften Wiederholung bestimmter verbaler Wendungen oder körperlicher Bewegungen einhergehen (Sandson \& Albert, 1984). Er beschreibt im Rahmen einer literarischen Erzähltextanalyse hingegen das unablässig wiederholte Artikulieren ein und derselben Wendungen und mehr oder weniger komplexer zusammenhängender Wortgruppen (Albes, 1999, S. 296). 
Beispiele für Textstellen, die eine solche beharrliche Wiederholung aufweisen, lassen sich in Gehen in vielen Passagen finden. So zum Beispiel in der Folgenden:

Keine Frage über Hollensteiner, sagt Oehler, keine Frage über die Umwelt Hollensteiners, keine Frage, die Stellung Hollensteiners in der heutigen Wissenschaft betreffend, keine Frage über die philosophischen Verhältnisse Hollensteiners, über seine Aufzeichnungen, geschweige denn über die Beziehung Hollensteiners zu Karrer, Karrers zu Hollensteiner. (GN, S. 52)

Die repetitive Namensnennung und die mehrfach eingebundene Phrase ,keine Frage“ verleihen der Passage einen bestimmten sprachlichen Rhythmus. Manfred Jurgensen spricht im Kontext seiner Überlegungen zu Gehen daher treffend von ,Sprachpartitur ${ }^{6}$ oder auch ,Sprachmusik“ (Jurgensen, 1981). Das Stilmittel der Perseveration manifestiert sich jedoch nicht nur in Form von Repetitionen, sondern auch in Form von Permutationen, also der wechselweisen Kombination einer geringen Anzahl von einzelnen Zeichen (Worten) untereinander. Darüber hinaus sind Verknüpfungen dieser beiden Strukturierungstechniken (Repetition und Permutation) an vielen Stellen zu finden. Ein Beispiel für eine permutierende Textpassage ist die Folgende:

Wenn ich gehe, sagt Oehler, denke ich und behaupte ich, ich gehe und auf einmal denke ich und behaupte ich, ich gehe und denke, weil ich das denke, während ich gehe. Und wenn wir zusammen gehen und diesen Gedanken denken, denken wir, wir gehen zusammen und auf einmal, wir denken, wenn auch nicht zusammen, wir denken, aber es ist etwas anderes. (GN, S. 88)

Die Personalpronomina ,ich “ und ,wir' und die Verben , gehen ' und ,denken' stehen hier in wechselnden Kombinationen zueinander. Durch die Kombination und Re-Kombination einzelner Signifikanten zu einem bestimmten Muster (Rhythmus) rücken die materiellen Eigenschaften der Sprache selbst an solchen Stellen in den Vordergrund und stehen damit für diesen Moment der Lektüre im Zentrum des kognitiven Rezeptionsprozesses.

Neben den eben beschriebenen rhythmischen Effekten nutzt Thomas Bernhard an anderen Stellen die klanglichen Qualitäten der ihm zur Verfügung stehenden Zeichen zur Schaffung ,lautlicher' Effekte. Die folgende kurze Passage zeigt vor allem beim lauten Lesen anschaulich, wie die Häufung von s- z- und sch-Lauten (und Abwandlungen davon) zusammengenommen eine Kakophonie bilden: „In Wirklichkeit aber ist sehr oft, was als tschechoslowakische Ausschußware bezeichnet wird und also, als solche von der Zollbehörde deklariert ist, erstklassigste englische oder eine andere erstklassigste ausländische Ware, so Rustenschacher zu Karrer“ (GN, S. 59). 
An anderer Stelle werden klangliche mit rhythmischen Sprachelementen verbunden und sogar ein Bezug zur semantischen Ebene - also dem geschilderten Geschehen - hergestellt. In der folgenden Passage liegt eine solche auffällige Emphase auf Klang und Rhythmus der Sprache. Der dichte sprachliche Rhythmus ,spiegelt' sich in Karrers rhythmischem Klopfen auf den Ladentisch des rustenschacherschen Ladens:

[...] dann hob Karrer wieder einmal den Stock und klopfte damit auf den Ladentisch und sagte: wahrscheinlich handelt es sich um einen Erschöpfungszustand, möglicherweise handelt es sich um einen Erschöpfungszustand, aber auf einen solchen Erschöpfungszustand kann ich keinerlei Rücksicht nehmen, keinerlei Rücksicht, sagte er sich, während er mit dem Stock auf den Ladentisch klopfte, in dem bestimmten Rhythmus, in welchem er immer auf den rustenschacherschen Ladentisch geklopft hat, [...]. (GN, S. 61)

Der selbstreflexive Charakter dieser Passage entsteht durch die Struktur-Semantik-Relation zwischen dem rhythmischen Klopfen mit dem Spazierstock und der allgemeinen rhythmischen Qualität der Sprachstruktur dieser Textstelle. Ein weiterer Struktur-Semantik-Bezug wird durch ein anderes Mittel hergestellt, wobei nicht die rhythmische Qualität der Sprache im Fokus steht, sondern das dominierende Darstellungsprinzip des Werkes, das Zitieren, selbst explizit benannt wird:

Dann vergeht einem alles, zitiert Oehler Karrer. Mir fällt auf, wie oft Oehler Karrer zitiert, ohne ausdrücklich darauf aufmerksam zu machen, daß er Karrer zitiert. Oft sagt Oehler mehrere von Karrer stammende Sätze und denkt sehr oft ein Karrer gedachtes Denken, denke ich, ohne ausdrücklich zu sagen, das, was ich jetzt sage, ist von Karrer, das, was ich jetzt denke, ist von Karrer. Im Grunde ist alles, was gesagt wird, zitiert, ist auch ein Satz von Karrer, der mir in diesem Zusammenhang einfällt und den Oehler sehr oft, wenn es ihm paßt, gebraucht. (GN, S. 22)

Das dominierende strukturelle Konstruktionsprinzip des Werkes ist das Zitat, wie Willi Huntemann richtig feststellt (Huntemann, 1990, S. 64). Dieses Strukturmerkmal wird an dieser Stelle ins Bewusstsein des Lesers gerückt, da eine der fiktiven Sprecherinstanzen (in diesem Fall der Ich-Erzähler) die Darstellungsform des Zitates durch die Wahl seiner Worte in den Vordergrund stellt. Der Erzähler weist explizit daraufhin, dass die durch Oehler wiedergegebenen Redeinhalte Karrers - in Übereinstimmung mit der gesamten formalen Gestaltung des Textes - zitiert sind. Das Prinzip dieser Darstellungsform erhält eine zusätzliche Emphase, da der Ich-Erzähler im Verlauf seines eigenen Kommentares in die Form des direkten Zitates wechselt, ohne dass dies durch eine entsprechende Zeichensetzung markiert wird. 
Die auffällige Verbindung zwischen Semantik und Struktur der aufgezählten Passagen und die teilweise gleichzeitige Unterbrechung des Ereignisberichtes begünstigt, dass sich die Aufmerksamkeit des Lesers auf formal-ästhetische Merkmale des Textes richten kann. Gleichzeitig werden solche Informationen einbezogen, die die Modalität der Informationsvermittlung und -aufnahme betreffen. Damit ist es wahrscheinlich, dass auch Informationen über die Beschaffenheit der aufgenommenen Informationen in die kognitive Verarbeitung einfließen. Solche kognitiven Informationen gehören wiederum einer anderen Kategorie an - oder anders gesagt, sie stehen auf einer anderen Ebene, als diejenigen, die dazu dienen, die geschilderte Ereigniskette kognitiv zu rekonstruieren und zu modellieren. Sie sind als MetaInformationen zu bezeichnen, da sie die Eigenschaften des Vermittlungsmodus kodieren also Informationen über die Beschaffenheit einer Informationsstruktur beinhalten. Die Verarbeitung von Meta-Informationen gehört ebenfalls zum Katalog der ToM-relevanten kognitiven Leistungen. Allerdings reichen die empirischen Ergebnisse, die im Zuge der ToMforschung gewonnen wurden nicht aus, um genau zu bestimmen, in welcher Form die Verarbeitung von Meta-Informationen hier in den kognitiven Leistungskatalog der ToM eingebunden ist. Perner (1991) und Leslie (1987) hatten argumentiert, dass die Verarbeitung von Meta-Informationen vor allem dann relevant ist, wenn die Funktion einer semantischen Information, bzw. ihre Stellung zur eigenen Wahrnehmung der Welt oder dem tatsächlichen Zustand der Welt gekennzeichnet werden muss. So kann der Inhalt einer Überzeugung (z. B. ,Es regnet') im Bezug zur realen Welt sowohl richtig als auch falsch sein. Wer über eine funktionierende ToM verfügt, ist in der Lage, diese Eigenschaft von Überzeugungen zu berücksichtigen, wenn sie eine bestimmte Situation interpretieren. Die Aufgabe lautet in einem solchen Fall den Bezug der (eigenen oder fremden) Überzeugung, nämlich dass es regnet, zur Realität herzustellen und damit diese Überzeugung entweder als falsch oder richtig anzuerkennen. Strittig ist, ob diese Prozesse bewusst oder unbewusst ablaufen und ob sich solche Leistungen auch über behavioristische Ansätze erklären lassen. Im Zuge verschiedener ToM-Aufgaben wie z. B. dem kindlichen Fiktionsspiel scheint lediglich die besondere Form der Verarbeitung Informationen durch einen Mechanismus zu sein, den Leslie ,Entkopplungsmechanismus' (Leslie, 1987) nennt. Ein solcher Mechanismus sorgt dafür, dass Informationen über eine fiktive oder imaginierte Situation entsprechend indiziert und klassifiziert werden. Auf diese Weise ist sichergestellt, dass die eigentliche Information über die zu repräsentierende reale oder fiktive Situation nicht durch die parallele Verarbeitung solcher Meta-Informationen gestört wird. Kinder sind damit in der Lage, z. B. im Zuge einer Episode des Fiktionsspiels, Informationen über die imaginierte Welt von Informationen über 
die reale Welt voneinander zu trennen. Sie können so zu jeder Zeit zuordnung, was in der imaginierten Welt der Fall ist und was in der realen Welt der Fall ist. Hier fehlt allerdings die Grundlage dafür, diese Informationsverarbeitung als Verarbeitung von Meta-Informationen also als Verarbeitung von Informationen über Informationen argumentativ zu stützen.

Wenn Leser einen fiktionalen Text lesen, müssen sie ebenfalls fiktionale von realen Inhalten von einander trennen können, um dem Text gegenüber eine angemessene Rezeptionshaltung einzunehmen. Darüber hinaus enthalten viele literarische Texte, wie Thomas Bernhards Gehen inhaltliche und strukturelle (,Meta'-) Bezüge zur eigenen Beschaffenheit. Solche Elemente halten Leser dazu an, neben der Rekonstruktion der Fiktionswelt auch Informationen über die Beschaffenheit und den Status der Inhalte, die sie im Verlauf der Lektüre vermittelt bekommen zu verarbeiten. Ob sich hier eine zentrale (und besondere) Parallele zwischen den kognitiven Leistungsanforderungen von ToM-relevanten Aufgaben und der kognitiven Rezeption literarischer Erzähltexte bennennen lässt, ist allerdings aus den oben genannten Gründen an dieser Stelle nicht endgültig zu klären. 



\section{Zusammenfassung}

Was ist die ToM? Im ersten Kapitel dieser Arbeit wurde mit Hilfe der bereits geleisteten Forschung aus der Entwicklungs- und Kognitionspsychologie eine allgemeine kognitive Fähigkeit des Menschen - die ToM - vorgestellt. Der Begriff ,ToM` bezeichnet (allgemein gesprochen) die Fähigkeit gesunder Erwachsener, sich selbst und anderen verschiedene mentale Haltungen zuzuschreiben und beobachtete Handlungen darauf basierend zu interpretieren oder zukünftige Ereignisse zu antizipieren. Die Entwicklung der hierfür notwendigen kognitiven Mechanismen im Zuge der Kindheit lässt sich anhand von neu hinzugewonnenen sozialen Kompetenzen und Verhaltensweisen nachvollziehen. Diese allgemeinen sozialen Kompetenzen sind im Rahmen überwiegend empirischer entwicklungs- und kognitionspsychologischer Arbeiten bereits gründlich untersucht worden. Diese Studien bildeten den Ausgangspunkt für ein tiefergehendes Verständnis der kognitiven Mechanismen und Ressourcen der ToM. Ziel des ersten Kapitels war es, an Hand der Beschreibungen empirischer Versuchsdesigns aus der kognitiven Entwicklungspsychologie diese Mechanismen zusammenzutragen und vorzustellen, um anschließend die Rolle dieser besonderen menschlichen Fähigkeit für die kognitive literarische Rezeption genauer, als es bisher geschehen ist, aufschlüsseln zu können.

Die ToM spielt in zahlreichen Bereichen der zwischenmenschlichen Interaktion eine wichtige Rolle. Die im ersten Kapitel dieser Arbeit gesammelten Informationen führten zu dem Schluss, dass die ToM in einen vielschichtigen Katalog unterschiedlicher sozialer Verstehensleistungen eingebunden ist. Mit anderen Worten: Ohne die ToM wäre eine Vielzahl typischer sozialer Interaktionsmuster des menschlichen Alltags nicht möglich. Dabei ist die ToM für unterschiedliche soziale Interaktionsmuster und interpretative Leistungen hilfreich. Auf der anderen Seite und im Hinblick auf bestimmte Verstehensleistungen (z. B. die Syn- 
chronisation von Wahrnehmung, das Fiktionsspiel, das Verständnis falscher Überzeugungen, das Verständnis uneigentlicher Kommunikation usw.) ist sie sogar eine notwendige Voraussetzung.

Mit Blick auf die unterschiedlichen Leistungen, die durch die ToM möglich sind, stellte sich die Frage, wie die ToM genau funktioniert oder mit anderen Worten: wie die Kognitionsprozesse der ToM zu charakterisierend sind. Diese Frage ist nicht neu und wurde bereits in vorangehenden, überwiegend theoretisch orientierten Arbeiten der Psychologie gestellt und diskutiert. Die wichtigsten Beschreibungsansätze, die ich in dieser Arbeit zusammengefasst habe, sind die Theorie der ,Meta'-kognition, die ,Theorie'-Theorie, die ,Simulations'-Theorie und die ,Narrativitäts'-Theorie. Das gemeinsame Ziel dieser Ansätze ist es, die Beschaffenheit der Denkprozesse, die der ToM zugrunde liegen, genauer zu definieren. In Auswertung der gewonnenen Erkenntnisse ist die Ansicht, die ToM sei in ihrer vielfältigen Rolle für die menschliche Kognition mit Hilfe eines einzigen dieser Ansätze zu erklären, meines Erachtens allerdings schwer zu begründen. Vielmehr lässt sich die Vielzahl unterschiedlicher sozialer und kognitiver Fähigkeiten sinnvoll durch das Zusammenspiel einer ganzen Reihe menschlicher kognitiver Mechanismen und Ressourcen erklären. Bei genauerer Betrachtung fiel zudem auf, dass sich die Hypothesen, die im Rahmen der verschiedenen Forschungsdiskurse zur Funktionsweise der ToM formuliert wurden, auf unterschiedliche Beschreibungsebenen der menschlichen Kognition beziehen. Sie verhalten sich daher zum Teil komplementär zueinander und sind an anderen Stellen schwer miteinander zu verbinden oder zu vergleichen. Die unterschiedlichen Ansätze, die sich innerhalb dieses Diskurses erkennen ließen, waren (ohne Rücksicht auf eine besondere Reihenfolge):

1. Die Erforschung hirnphysiologischer Mechanismen (z. B. Spiegelneuronen) als Grundlage für die sozialen Leistungen, die der ToM zugeschrieben werden.

2. Funktionale Beschreibungen von ToM-spezifischen kognitiven Mechanismen (z. B. der ,Entkopplungsmechanismus'), die für die beobachtbare Entwicklung sozialer Kompetenzen notwendig sind.

3. Die Suche nach einem Zusammenhang mit allgemeinen Prinzipien und Konzepten der menschlichen Kognition, die allen kognitiven Prozessen und damit auch der ToM zugrunde liegen (z. B. die Repräsentation von Informationen mittels eines kognitiven ,Codes` und mentaler Modelle, das Arbeitsgedächtnis, die Inhibitorische Kontrolle, lexikalische und syntaktische Kompetenzen). 
4. Die Beschreibung und Charakterisierung von heuristischen Strategien zur Wahrnehmung und Evaluation von situationsrelevanten mentalen Haltungen (z. B. (a) die ,Simulation' fremder mentaler Vorgänge mit Hilfe der eigenen Erfahrungen, oder (b) die Fähigkeit, mentale Kontexte mit Hilfe einer ,naiven Theorie“ - also mit Hilfe von abstraktem Wissen über die Funktion und Bedeutung von mentalen Haltungen - zu erschließen, oder (c) Metakognition und die Fähigkeit, die Bedeutung von verschiedenen Kategorien mentaler Haltungen zu reflektieren)

5. Die Beschreibung von Denkprozessen zur kognitiven Situationsrekonstruktion (z. B. Fähigkeit zur Herleitung motivationaler Zusammenhänge dank ,narrativer' Informationsstrukturierung).

Die Vielzahl unterschiedlicher ToM-relevanter Verstehensleistungen lassen sich damit erklären, dass simulative, abstrahierende, metakognitive und integrative Kognitionsprozesse situationsabhängig zusammenwirken und sich dadurch ergänzen. Wie bereits erwähnt ist die Synthese aller im ersten Arbeitsteil vorgestellten Ansätze im Sinne einer umfassenden Definition des ToM-Begriffes aufgrund der unterschiedlichen Beschreibungsebenen schwierig. Als Fazit des ersten Kapitels ist daher folgendes festzuhalten: Es ist wahrscheinlich, dass die Fähigkeiten, die uns unter dem Dach des ,ToM`- Begriffes zuzusprechen sind, durch das (situationsabhängige) Zusammenspiel vieler kognitiver Funktionen bzw. Mechanismen ermöglicht wird.

Neben der ToM gehört auch die literarische Textrezeption zu den besonderen kognitiven Fähigkeiten des Menschen. Eine der zentralen Aufgaben bestand darin, herauszufinden, ob es einen Zusammenhang zwischen diesen zwei besonderen kognitiven Kompetenzen gibt und wie dieser Zusammenhang zu charakterisieren ist. Nachdem das ToM-Konzept im ersten Kapitel nach einzelnen zentralen Kognitionsmechanismen aufgeschlüsselt wurde, galt es im Anschluss zu prüfen, ob sich präzisere Aussagen über den Zusammenhang dieser Mechanismen und der kognitiven literarischen Textrezeption formulieren lassen, als es im gegenwärtigen literaturwissenschaftlichen Forschungsdiskurs bisher geschehen ist. Um erkennen zu können, welche Rolle diese Mechanismen in der Rezeption eines literarischen Erzähltextes spielen, habe ich mich im Verlauf meiner Argumentation auf die funktionalen kognitiven Mechanismen konzentriert, die für die unterschiedlichen sozialen Fähigkeiten relevant sind. Der Begriff , ToM` wurde im weiteren Verlauf dieser Arbeit daher lediglich zur Bezeichnung einer allgemeinen Fähigkeit verwendet - nämlich derjenigen, sich selbst 
und anderen innere (z. B. perzeptive oder emotionale) Vorgänge oder mentale Haltungen (z. B. Intentionen, Überzeugungen oder Wünsche) zuzuschreiben.

Im Sinne dieser Aufgabenstellung war es notwendig, herauszufinden, ob es einen kognitiven ,common ground ${ }^{\star}$ zwischen den Kognitionsmechanismen der ToM und denjenigen der kognitiven Textrezeption gibt. Hierfür war ein Vergleich der Leistungsanforderungen dieser zwei unterschiedlichen Fähigkeiten notwendig. Menschen verarbeiten sowohl in ihrem sozialen Umfeld als auch während der Lektüre eines literarischen Textes eine bestimmte Menge bestimmter Informationen, die ihnen in bestimmter Art und Weise und Reihenfolge zugeführt werden. Meine Argumentation gründete darauf, dass der Interpretationsprozess eines Lesers, ebenso wie Interpretationsprozesse im sozialen Kontext, durch das jeweils gegebene System von Informationen gelenkt wird. Durch diese Herangehensweise war es möglich, die Funktionen der im ersten Kapitel beschriebenen Kognitionsmechanismen im Rahmen der literarischen Rezeption genauer zu beschreiben. Es ließ sich auf diesem Weg zunächst plausibel begründen, warum Menschen ihre ToM-relevanten kognitiven Mechanismen nicht nur im Rahmen der sozialen Interaktion, sondern auch für die Interpretation literarisch vermittelter Handlungen nutzen können. Ein Blick auf allgemeine bzw. typische Merkmale literarischer Erzähltexte zeigte, dass trotz der oberflächlichen Unterschiede in der Informationsvermittlung dennoch die Grundlage für die Bildung mentaler Situationsmodelle im Zuge der Textrezeption gegeben ist. Damit lässt sich sinnvoll begründen, warum und inwiefern sowohl im Zuge der sozialen Interaktion, als auch zugunsten der Interpretation literarisch dargestellter Handlungen vergleichbare kognitive Prozesse mit Hilfe der ToMmechanismen ablaufen können. Die grundlegende Annahme lautete dabei: Sollten sich strukturelle Parallelen der Informationssysteme finden lassen, ist der Schluss zu rechtfertigen, dass vergleichbare kognitive Prozesse bzw. dieselben (ToM-relevanten) kognitiven Ressourcen und Mechanismen für die Verarbeitung einer Informationsgruppe erforderlich sind. Meine Schlussfolgerung lautet, dass beide Fähigkeiten - die ToM und die literarische Textrezeption - sich durch eine Reihe strukturell vergleichbarer kognitiver Leistungsanforderungen auszeichnen. Es ist an solchen Stellen der vergleichbaren Leistungsanforderung gerechtfertigt anzunehmen, dass die Informationsverarbeitung durch dieselben kognitiven Mechanismen getragen wird.

Um die Berührungspunkte zwischen den beiden kognitiven Interpretationsvorgängen zu identifizieren, dienten ausgewählte zentrale Merkmale literarischer Erzähltexte (z. B. ihre 
narrative Informationsstruktur, ihre sprachlichen und fiktionalen Eigenschaften und mögliche Merkmale der Literarizität) zur Orientierung. Es stellte sich heraus, dass die Attribution von mentalen Haltungen und inneren Vorgänge gegenüber fiktiven Figuren und Autoren nur eine von mehreren kognitiven ToM-relevanten Leistungen ist, die Leser im Zuge der Rezeption eines literarischen Textes erbringen müssen. Es ließen sich darüber hinaus eine Vielzahl von Berührungspunkten zwischen den kognitiven Anforderungen der Lektüre eines literarischen Erzähltextes und den Interpretationsleistungen im sozialen Kontext benennen. Sie sprechen für eine zentrale Funktionen dieser Gruppe ToM-relevanter kognitiver Mechanismen im Zuge der literarischen Rezeption:

1. Die ToM übernimmt eine wichtige Funktion, wenn es darum geht, Aussage- und Handlungsintentionen richtig zu interpretieren. Im sozialen Kontext ist dies vor allem dann relevant, wenn Handlungen im Rahmen des Fiktionsspiels als solche interpretiert werden müssen, die sich nicht in direkter Form auf die Realität beziehen. Diese Funktion ist auch relevant, um Lügen als solche zu erkennen und bestimmte Formen der uneigentlichen Kommunikation (z. B. Ironie/Sarkasmus, Metaphern etc.) zu verstehen. Im Zuge der literarischen Rezeption ist diese Funktion vor allem dann entscheidend, wenn ein Leser vor der Aufgabe steht, Aussagen innerhalb eines literarischen Erzähltext in ein adäquates Verhältnis zur Realität zu stellen. Sie müssen solchen Aussagen mit anderen Worten mit Hilfe ihrer ToM interpretationsrelevante Aussageintentionen zuweisen.

2. Wie in den Arbeiten von Zunshine (2006; 2010) und Palmer (2004) ausführlich beschrieben, müssen Leser darüber hinaus literarisch dargestellte, zielgerichtet handelnde Figuren und Handlungen kognitiv repräsentieren. Die Interpretation literarisch dargestellter Handlungen erfordert - ähnlich wie im realen sozialen Kontext - in vielen Fällen die kognitive Attribution mentaler Haltungen, um motivationale Zusammenhänge zu erschließen. Leser können ihre ToM für die Interpretation literarisch dargestellter Haltungen ebenso wie für die Interpretation realer beobachteter Handlungen einsetzen. Diese These ließ sich mit einem Blick auf wichtige grundlegende Prinzipien der menschlichen Kognition stärken. Trotz der vielen und zum Teil großen Unterschiede zwischen der Beschaffenheit und der Art der Vermittlung von Informationen, entstehen in beiden Fällen dank der kognitiven Modellierung von Situationsmodellen vergleichbare Voraussetzung für interpretative Schlüsse. Leser können in beiden Fällen die ge- 
gebenen Informationen, die sich auf Ereignisse, Personen und deren Relationen zueinander beziehen, zu einer Reihe von Situationsrepräsentationen synthetisieren, mit deren Hilfe prädiktive Schlüsse über zukünftige Handlungen, Ereignisse und Handlungsziele und rückblickende Schlüsse über handlungsmotivierende mentale Haltungen gezogen werden können.

3. Um einen Erzähltext, in welchem Informationen auf narrativem Weg vermittelt werden, richtig zu verstehen, müssen Leser in der Regel an Hand deiktischer Hinweise Wahrnehmungsstandpunkte und Perspektiven rekonstruieren. Es ist für ein gelingendes Textverständnis ausschlaggebend, diese Wahrnehmungsstandpunkte und ihre Relation zum deiktischen Gefüge der Fiktionswelt zu berücksichtigen. Menschen besitzen die Fähigkeit, Informationen nicht allein mit Bezug zu ihrem expliziten semantischen Gehalt oder zur bekannten Realität zu interpretieren, sondern können sie mit Bezug auf einen bestimmten subjektiven (mentalen und lokalen) Standpunkt rekonstruieren. Damit sind geeignete kognitive Ressourcen gegeben, um Informationen verarbeiten zu können, die die grundlegende Konfiguration der Erzählsituation und die Fokalisierungsstrategie des Textes betreffen. Fokalisierungsstrategien halten Leser dazu an, literarisch dargestellte Situationen aus einem bestimmten (ggf. in bestimmter Art und Weise begrenzten) Blickfeld zu rekonstruieren. In Abhängigkeit davon müssen weitere interpretationsrelevante Informationen erst aktiv durch den Leser erschlossen werden, um zu einem kohärenten Situationsmodell zu gelangen. Es sind im Fall literarischer Texte die deiktischen Mikrostrukturen, die Leser in diesem Sinne wie kognitive ,Fingerzeige‘ anleiten, die dargestellten Situationen entsprechend zu rekonstruieren.

4. Die Informationen innerhalb eines Erzähltextes sind nicht als Wahrnehmungseindrücke, sondern als narrativ vermittelte Informationen zu klassifizieren. Diese auf den ersten Blick triviale Einsicht ist wichtig, denn mit einem Blick auf die Eigenschaften eines Vermittlungsmodus lassen sich Schlüsse über die Art und Anzahl der an der Interpretationsaufbereitung und -verarbeitung beteiligten kognitiven Mechanismen ziehen. Es ist eine grundlegende Eigenschaft des narrativen Vermittlungsmodus, dass die so vermittelten Informationen in eine narrative Handlung eingebunden sind. Die erste Ebene realer Interaktionen ist die Ebene der direkten Wahrnehmung. Wenn im Zuge einfacher realer Interaktionen etwas geschieht, dann ist dies (auf erster und einfachster Ebene) auf direktem Weg über die Sinnesorgane (Augen, Ohren, Nase etc.) erfahrbar. Diese Ebene ist vergleichbar mit der realen Lektüreerfahrung empirischer Leser, die ein Buch in einer 
bestimmten Umgebung in der Hand halten, Buchstaben sehen etc. Um die Fiktionswelt zu erschließen, müssen Leser erst rekonstruieren, was über diese Welt gesagt wird. Mit anderen Worten: Während in der realen Welt etwas geschieht, muss innerhalb eines Erzähltextes über eine Fiktionswelt erst gesagt (bzw. geschrieben) werden, dass etwas geschieht. Damit müssen hier bereits auf erster Rekonstruktionsebene eingebettete Informationsvermittlungsstrukturen verarbeitet werden. Narrative Akte teilen ihre grundlegende kognitiv/grammatische Beschaffenheit mit derjenigen von Komplementsätze. Hierfür ist jeweils ein kognitiver Mechanismus zur Verarbeitung eingebetteter Informationsstrukturen relevant. Er spielt sowohl für ein Verständnis mentaler Haltungen als auch für die Verarbeitung narrativ vermittelter Informationen eine wichtige Rolle, da in beiden Fällen eingebettete Informationen in Relation zu ihrer Funktion (einer bestimmten mentalen Haltung oder dem narrativen Akt) interpretiert werden müssen.

5. Leser können die im Zuge der Lektüre fiktionaler Texte die dargestellten Ereignisse außerdem mit Hilfe der dafür zuständigen kognitiven Ressourcen imaginieren. Hierfür ist ein kognitiver Mechanismus zentral, der in der Entwicklungspsychologie funktional als ,Entkopplungsmechanismus' bezeichnet wurde. Er stellt sicher, dass Informationen über fiktive und reale Gegenstände - und die auf sie bezogenen emotionalen Reaktionen - nicht miteinander konkurrieren. Dieser Mechanismus ist auch für das kindliche Fiktionsspiel und die Trennung eigener und fremder mentaler Haltungen relevant.

6. Es ist abschließend auch wahrscheinlich, dass Leser während der Lektüre eines literarischen Textes auch Informationen über die formalen Besonderheiten (z. B. das verwendete Sprachsystem), sonstige strukturelle und ästhetische Eigenschaften und über den Vermittlungsmodus des Textes kognitiv verarbeiten. Neben einfachen metalinguistischen Informationen können im Fall von literarischen Erzähltexten, die nicht nur sprachlich vermittelt sind, sondern ihre ästhetischen Besonderheiten z. T. mit verschiedenen Mitteln in den Vordergrund stellen, auch meta-poetische Repräsentationen in die kognitive Rezeption einfließen. Das ist vor allem dann wahrscheinlich, wenn die linguistischen Eigenschaften von Texten deutlich von den gewohnten Sprachsystemen (z. B. demjenigen der Alltagssprache) abweichen. Auch hier ließ sich eine Parallele zur Verarbeitung von Informationen im Kontext von ToM-relevanten Aufgaben ziehen. Dort war - wie im ersten Teil der Arbeit näher erläutert - die Bildung von Repräsentationen unterschiedlicher Abstufung (im Sinne von ,Meta'-Repräsentationen) auch eine zentrale 
Voraussetzung um z. B. die Funktion und Unterschiede verschiedener Kategorien mentaler Haltungen (von etwas überzeugt sein vs. etwas wissen vs. etwas intendieren etc.), oder auch um den Modus von Fiktionsrepräsentationen anzuerkennen.

Die hier zusammengefassten Erkenntnisse legen nahe, dass die beiden Fähigkeiten der ToM und der literarischen Textrezeption zahlreiche kognitive Verbindungspunkte aufweisen. Sie werden damit zumindest zum Teil von denselben kognitiven Mechanismen gestützt. Diese These konnte ich im zweiten Teil meiner Arbeit mit Hilfe eines Vergleiches der jeweils involvierten funktionalen kognitiven Leistungsanforderungen stärken. Gleichzeitig konnte ich - ohne Anspruch auf Vollständigkeit erheben zu wollen - eine Reihe relevanter kognitiver Mechanismen, die für beide Fähigkeiten zentral sind, eingrenzen.

Im Anschluss an diese Überlegungen lag der Fokus auf den individuellen Strukturen und Merkmalen einzelner Werke. In diesem Kontext galt es, sich zwei unterschiedlichen Aufgaben zu stellen. Es sollte erstens gezeigt werden, dass sich mit dem Wissen um die besonderen Merkmale individueller Texte und dem Wissen um die Funktionsweise der ToM sinnvolle Hypothesen über die kognitiven Rezeptionsprozesse in Bezug auf einen bestimmten Text formulieren lassen. Hier ist die ToM nicht nur Teil des Untersuchungsgegenstandes, sondern auch das leitende Prinzip der Untersuchung, da einem (Modell-) Leser basierend auf einer Reihe beobachtbarer Variablen (der konkreten Textkonfiguration im Einzelfall) kognitive Vorgänge zugeschrieben wurden. Die in den ersten beiden Teilen dieser Arbeit gewonnenen Erkenntnisse über den Katalog ToM-relevanter kognitiver Mechanismen und ihre Funktion für die literarische Textrezeption dienten hier als Richtlinien. Aus methodischer Sicht möchte ich abschließend festhalten, dass sich die Bedingungen für die spezifischen Kognitionsprozesse, die während der Lektüre eines bestimmten Textes ablaufen, mittels einer methodischen Textanalyse genauer charakterisieren ließen. Mein Fazit lautet, dass es basierend auf der Analyse eines Textes nach narratologischen, strukturellen und semantischen Merkmalen möglich ist, die Leistungsanforderungen eines Textes mit den kognitiven Leistungsanforderungen unserer sozialen Umwelt abzugleichen. Ein solcher Vergleich wiederum bildet ein geeignetes Fundament, um Hypothesen darüber zu formulieren, welche kognitiven Mechanismen in die Lektüre eines bestimmten literarischen Textes eingebunden sind und inwiefern die Merkmale des Textes die Rezeptionshaltung und Interpretationsprozesse eines Lesers beeinflussen bzw. lenken. Mit Hilfe des Wissens über verschiedene Prinzipien und 
Eigenschaften der menschlichen Kognition, welches in der Vergangenheit aus der Beobachtung realer Interaktionen gewonnen wurde, habe ich (nach dem Attributionsprinzip der ToM) einem (Modell-) Leser ein bestimmtes Rezeptionsverhalten zugeschrieben.

Diese Vorgehensweise führte zu interessanten Einblicken in die kognitiven Leistungsanforderungen der einzelnen Werke. Mit ihr ging auch die Frage einher, ob ein Vergleich zweier völlig unterschiedlicher Werke hinsichtlich der Analyse ihrer kognitiven Rezeptionsanforderungen (in dieser Arbeit mit besonderem Bezug zu ToM-relevanten Mechanismen) möglich und sinnvoll ist. Anhaltspunkte für diese Untersuchung war einerseits die Analyse vergleichbarer Merkmale wie z. B. die grundlegende narrative Beschaffenheit der beiden Texte als auch die jeweils besonders auffälligen bzw. charakteristischen Merkmale der einzelnen Werke. Um diese charakteristischen Merkmale zu identifizieren, habe ich mich an den im jeweils relevanten Forschungsdiskurs häufig angesprochen Werkmerkmalen orientiert: im Fall der Njáls Saga sind dies z. B. der umstrittene Fiktionsstatus der geschilderten Ereignisse, die besondere Konfiguration der Erzählsituation sowie die Kunstgriffe, die Verwendung finden, um die Vielzahl der geschilderten Ereignisse kohärent in die Erzählung einzubinden. Im Fall von Gehen sind es u. a. die komplexe Darstellungsweise der Ereignisse, die durch die gewählte gestaffelte Gesprächssituation entsteht, und die besonders auffällige Selbstreflexivität und Poetizität des Textes. Im hier gewählten Vergleich dieser zwei Texte ließen sich trotz der großen individuellen Merkmalsdifferenzen einige Parallelen in den grundlegenden kognitiven Leistungsanforderungen an den Leser identifizieren. Ich gehe daher davon aus, dass es eine Reihe von ToM-relevanten Kognitionsmechanismen gibt, die trotz der oberflächlichen Unterschiede für die Lektüre beider Werke relevant sind. Damit sind 1. diejenigen Mechanismen gemeint, die für die Informationsrestrukturierung zuständig sind. Diese Restrukturierung ist notwendig, um die durch einen Text in einer bestimmten Reihenfolge vermittelten Informationen kognitiv so zu ordnen, dass die dargestellte fiktive Situation kognitiv modelliert werden kann. Damit sind 2. Mechanismen gemeint, die einem Leser gestatten, im Zuge von Interpretationsprozessen die unterschiedlichen ,Wahrnehmungsstandpunkte“ innerhalb einer Fiktionswelt und der narrativen Vermittlung der Informationen zu berücksichtigen. Hier ist es notwendig, die verschiedenen Hinweise innerhalb eines deiktischen Informationssystems für die Evaluation der gegebenen Informationen zu nutzen, um bestimmen zu können, wer die Information aus welcher Perspektive und mit welcher (z.B. emotionalen oder intentionalen) Haltung vermittelt. Beide Werke enthalten außerdem 3. Passagen, die den Leser mit ambivalenten Interpretationsmöglichkeiten kon- 
frontieren. Beide Texte halten 4. ihre Leser dazu an, verschiedene miteinander zusammenhängende fiktive Situationen zeitdeckend während der Lektüre einer bestimmten Textpassage zu rekonstruieren. Im Fall der Njáls Saga wurde dies durch eine prophetische/anweisende Erzählstrategie und im Fall von Gehen durch die ineinander gestaffelte Wiedergabe von Gesprächsinhalten realisiert. Nicht zuletzt verwiesen 5. bestimmte semantische oder strukturelle Elemente der Textgestaltung beider Werke explizit oder implizit auf die jeweils eigene Beschaffenheit des Textes bzw. den gewählten Modus der Darstellung von erzählten Ereignissen und Figuren. Daher gehe ich davon aus, dass in beiden Fällen Repräsentationen zweiter Stufe oder sekundäre Repräsentationen in den Interpretationsprozess einfließen.

Bei einem Blick auf die beiden individuellen Werke im Vergleich ist festzuhalten, dass sich Thomas Bernhards Kurprosastück auf den ersten Blick deutlich von der Njáls Saga unterscheidet. Beide Texte unterscheiden sich hinsichtlich ihrer Länge, Komplexität und Editionsgeschichte. Aufbauend auf die Erkenntnisse des zweiten Arbeitskapitels war es nicht überraschend, dass bestimmte ToM-relevante kognitive Ressourcen in beiden Fällen erforderlich sind, um zu einem kohärenten Textverständnis zu gelangen. Darüber hinaus zeigte sich bei genauerem Hinsehen, dass neben den erwarteten Parallelen auch durch unterschiedlicher Merkmale vergleichbare kognitive Rezeptionsprozesse stimuliert werden. Während einerseits gemeinsame grundlegende und typische Merkmalen narrativer literarischer Texte vergleichbare Informationsverarbeitungsprozesse begründen, stimulieren beide Texte auffälliger Weise parallele kognitive Leistungen mit Hilfe unterschiedlicher Darstellungsformen und Vermittlungsmethoden. So waren Leser in beiden Fällen dazu angehalten, aufgrund ineinander geschachtelter Referenzen auf zwei verschiedene Situationen, diese zwei Situationen fortlaufend zu modellieren und parallel zueinander zu repräsentieren. In der Njáls Saga geschah dies durch die vorausblickende Beschreibung der Täuschung von Hrútur durch Gunnar. In Thomas Bernhards Werk wird eine solche kognitive Leistung durch die geschachtelte Erzählung eines Gespräches in Form eines Gespräches realisiert. Außerdem verweisen beide Texte (erneut in deutlich unterschiedlicher Art und Weise) auf ihre eigene Vermittlungsmodalität. So ist die Erzählhaltung der Njáls Saga u. a. durch häufige Verweise auf eine (vorgegebene) Historizität des Geschehens gekennzeichnet. Thomas Bernhards Erzählstück zeichnet sich durch eine in den Vordergrund gestellte Materialität der Sprache und selbstreflexive Bezüge aus.

Die beiden hier geleisteten Textanalysen haben gezeigt, dass trotz der Unterschiedlichkeit der gewählten Werke in beiden Fällen vergleichbare kognitive Mechanismen angesprochen 
werden. Eine solches Ergebnis ist deshalb interessant, weil sich die individuelle Gestaltung und die narrativen Strategien, die diese Prozesse erforderlich machen, in den beiden vorliegenden Fällen deutlich voneinander unterscheiden. Die in dieser Arbeit zur Untersuchung der textspezifischen Fragen gewählte Methode basiert auf einer Analyse der Informationsstruktur des jeweiligen Textes. Die Ergebnisse dieser Analyse bilden wiederum die Grundlage für eine Attribution von interpretativen Prozessen gegenüber einem Modell-Leser. Auf diese Weise konnten Aussagen über die kognitiven Leistungsanforderungen der einzelnen Texte gemacht werden und die Abläufe kognitiver Prozesse miteinander verglichen werden. 


\section{Ausblick}

Die Argumentation und Schlussfolgerungen in dieser Arbeit gründen hauptsächlich auf den auf empirischem Weg gesammelten Daten und theoretischen Beschreibungsansätze der Kognitionspsychologie. Auf diesem Fundament ließ sich ein Arbeitskonzept der ToM erstellen, das allen weiteren Überlegungen dieser Arbeit zugrunde lag. Es darf dabei nicht in Vergessenheit geraten, dass die ToM im ursprünglichen kognitionswissenschaftlichen Diskurs noch nicht endgültig erfasst ist. Das betrifft vor allem die exakte Bestimmung derjenigen kognitiven Mechanismen und Ressourcen, die notwendig sind, um die mit der ToM assoziierten Leistungen gesunder Erwachsener zu ermöglichen. Diese kognitiven Ressourcen und Mechanismen müssen noch präziser untersucht und bestimmt werden, bevor in $\mathrm{Zu}$ kunft im Kontext rezeptionstheoretischer Arbeiten noch genauere Hypothesen formuliert werden können, als es hier möglich war.

Trotzdem eine gründliche Erforschung der zugrundeliegenden kognitiven Mechanismen noch aussteht, war es hier bereits möglich, die funktionalen kognitiven Voraussetzungen für die mit der ToM assoziierten Fähigkeiten zu beschreiben. Dies bildete eine geeignete Argumentationsgrundlage für den Vergleich mit den kognitiven Leistungsanforderungen, die im Rahmen der literarischen Rezeption entstehen. Der Fokus meiner Abriet lag dabei auf einer bestimmten Gattung, nämlich derjenigen literarischer Erzähltexte. Im Anschluss daran wäre ein ebensolcher allgemeiner Vergleich kognitiver Anforderungen mit Blick auf Werke anderer Gattungen (aus den Bereichen der Lyrik und Dramatik) denkbar. Hier wäre es spannend zu klären, ob und inwiefern Texte unterschiedlicher Gattungen aufgrund ihrer typischen Gattungsmerkmale zuverlässig bestimmte kognitive Mechanismen stimulieren. Lassen sich ggf. sogar gattungsspezifische Unterschiede in der kognitiven Rezeption ausmachen? Möglicherweise lässt sich unter einer anderen Perspektive begründen, dass bestimmte Textgattungen und Genres die ToM nicht nur in unterschiedlicher Form stimulieren. Denkbar ist ebenfalls, dass sie zwar in jedem Fall vergleichbare kognitive Mechanismen aktivieren, diese aber in unterschiedlich intensiver Form stimulieren? 
Ähnliche Fragen wären ebenfalls unter einem literarhistorischen Blickwinkel zu stellen. Möglicherweise lassen sich bestimmte epochenspezifische Poetiken identifizieren, die besondere kognitive Leistungsanforderungen mit sich bringen. Eine solche Untersuchung ließe sich auf zwei Wegen leisten. Zum einen wäre es möglich, sich an den bereits in vorangehenden literarhistorischen und gattungstheoretischen Arbeiten gewonnenen Erkenntnissen über typischen Merkmalen von Texten eines Genres, einer Gattung oder einer Epoche zu orientieren und Hypothesen über die kognitiven Anforderungen die mit diesen Merkmalen einhergehen, aufzustellen (top down). Es ist ebenfalls denkbar, aufbauend auf die Methodik des dritten Hauptteils dieser Arbeit eine repräsentative Stichprobe von Texten einer Gattung oder einer Epoche miteinander zu vergleichen und nach Gemeinsamkeiten und Unterschieden in den kognitiven Leistungsanforderungen zu suchen (bottom up).

Der explorative Vergleich zweier sehr unterschiedlicher Werke in dieser Arbeit bestätigte bereits die intuitive Überlegung, dass unterschiedliche literarische Texte von ihren Lesern zum Teil unterschiedliche kognitive Prozesse während des Interpretationsprozesses erfordern. Gleichzeitig ließen sich aber auch Parallelen identifizieren. Es stellte sich heraus, dass durch unterschiedliche gestalterische Strategien ebenfalls eine Reihe vergleichbarer ToMrelevanter kognitiver Prozesse stimuliert wird. Sind genau diese Prozesse für die Rezeption literarischer Texte im Allgemeinen von besonderer Bedeutung? Lässt sich eventuell zukünftig begründen, dass bestimmte kognitive Prozesse für die Rezeption von Texten einer bestimmten Gattung oder Epoche oder gar für die Rezeption literarischer Texte im Allgemeinen konstitutiv sind?

Eine weitere Frage, die in dieser Arbeit nicht gestellt wurde und damit offenbleibt, ist fundamentalerer Natur: Literarische Texte werden - zumindest bisher - üblicherweise von Menschen geschaffen. Wird die Beschaffenheit von literarischen Erzähltexten (oder Kunstwerken im Allgemeinen), die von Menschen geschaffen werden, in diesem Fall nicht bereits durch dieselben Prinzipien menschlicher Kognition pre-determiniert? Ist die ToM deshalb auf fundamentaler Ebene in die literarische Rezeption involviert, weil sie zum grundlegenden kognitiven Repertoire von Autoren und Lesern gleichermaßen gehört? Kann es also literarische Erzähltexte geben, deren Rezeption die ToM-relevanten kognitiven Mechanismen nicht involviert? Diese Fragen sind grundlegender Natur und ihre Klärung erfordert weitreichende Studien, die Werke unterschiedlicher Gattungen und Epochen in die Untersuchung einbeziehen. Ebenso wie ein Vergleich der kognitiven Leistungsanforderungen von Texten unterschiedlicher literarischer Gattungen und Epochen, ist ein Vergleich unterschiedlicher 
Kunstrichtungen (Architektur vs. Bildende Kunst) hinsichtlich ihrer typischen oder individuellen Rezeptionsbedingungen spannend. Hier ließe sich klären, ob und in welcher Form die ToM-relevanten Mechanismen für die Kunstrezeption im Allgemeinen relevant sind.

Die vorliegende Arbeit legt das Fundament für solche weiterführenden Untersuchungen. Auf diesem Fundament steht einerseits die Erkenntnis, dass sich Arbeiten, die die besondere Gruppe ToM-relevanter kognitiver Mechanismen in den Fokus stellen, nicht auf die allgemeine Funktion der ToM für die Rekonstruktion motivationaler Handlungszusammenhänge beschränken müssen. Vielmehr ist es möglich, durch die Aufschlüsselung des allgemeinen Begriffes der ToM detailliertere Erkenntnisse zu gewinnen. Die Analyse und der Vergleich von Informationsstrukturen und der Rückschluss auf durch sie aktivierte kognitive Verstehensprozesse bilden anschließend eine geeignete Methode, um die kognitive Rezeption von literarischen Erzähltexten (und weiterführend anderen Kunstwerken nach demselben Prinzip) genauer zu erfassen.

Auf der anderen Seite darf nicht vergessen werden, dass die Überlegungen und Schlussfolgerungen dieser Arbeit auf einem Arbeitskonzept der ToM basieren. Wie die ToM tatsächlich funktioniert, d. h. mittels welcher konkreten kognitiven Prozesse und Mechanismen Menschen über die entsprechenden Fähigkeiten verfügen können, kann hier nur funktional beschrieben, nicht aber abschließend definiert werden. Eine solche funktionale Beschreibung der relevanten kognitiven Mechanismen ist mit einem Blick auf die sichtbaren Verhaltensweisen und Fähigkeiten von Menschen in sozialen Interaktionen möglich. Allerdings geht damit kein Anspruch auf eine erschöpfende Darstellung der ToM einher. Entsprechend richtet sich der Fokus im Rahmen der Analyse allgemeiner und spezifischer Textmerkmale auf Zusammenhänge mit denjenigen kognitiven Mechanismen, die hier für die ToM als relevant erachtet wurden. Zukünftige Arbeiten können von zukünftigen, noch detaillierteren Erkenntnissen über die Funktionsweise der ToM profitieren. Solche Erkenntnisse würden die Grundlage dafür legen, die Rolle der ToM im Zuge der Rezeption literarischer Erzähltexte und anderer Kunstformen noch präziser zu erfassen, als es im Rahmen dieser Arbeit möglich war. 


\section{Literaturverzeichnis}

\section{Sekundärliteratur}

Albes, C. (1999). Der Spaziergang als Erzählmodell. Studien zu Jean-Jacques Rousseau, Adalbert Stifter, Robert Walser und Thomas Bernhard. Tübingen [u. a.]: Francke Verlag.

Alicke, M. D., \& Largo, E. (1995). The role of self in the false consensus effect. Journal of Experimental Social Psychology,31(1), 28-47.

Allen, R. F. (1971). Fire and iron: critical approaches to njáls saga. Pittsburgh (PA): University of Pittsburgh Press.

Astington, J. W., \& Jenkins, J. M. (1999). A longitudinal study of the relation between language and theory-of-mind development. Developmental Psychology, 35(5), 1311-1320.

Baron-Cohen, S. (1997). Mindblindness: An essay on autism and theory of mind. Cambridge (MA): MIT press.

Baron-Cohen, S., Leslie, A. M., \& Frith, U. (1985). Does the autistic child have a ,theory of mind"? Cognition, 21(1), 37-46.

Billenkamp, M. (2008). Thomas Bernhard: Narrativik und poetologische Praxis. Heidelberg: Universitätsverlag Winter.

Booth, W. C. (1961). The rhetoric of fiction. Chicago: University of Chicago Press.

Bruner, J. S. (1986). Actual minds, possible worlds. Boston (MA): Harvard University Press.

Bruner, J. S. (1990). Acts of meaning. Boston (MA): Harvard University Press.

Buccino, G., Binkofski, F., Fink, G. R., Fadiga, L., Fogassi, L., Gallese, V., Seitz, R. J., Zilles, K., Rizzolatti, G. \& Freund, H. J. (2001). Action observation activates premotor and parietal areas in a somatotopic manner: an fMRI study. European Journal of Neuroscience, 13(2), 400-404.

Caramazza, A., Anzellotti, S., Strnad, L., \& Lingnau, A. (2014). Embodied cognition and mirror neurons: a critical assessment. Annual Review of Neuroscience, 37, 1-15. 
Carlson, S. M., Moses, L. J., \& Claxton, L. J. (2004). Individual differences in executive functioning and theory of mind: An investigation of inhibitory control and planning ability. Journal of Experimental Child Psychology, 87(4), 299-319.

Carruthers, P. (1996). Simulation and self-knowledge: a defence of theory-theory. In P. Carruthers und P. K. Smith (Hrsg.), Theories of Theories of Mind (22-38). Cambridge University Press.

Charman, T., \& Baron-Cohen, S. (1997). Brief report: Prompted pretend play in autism. Journal of Autism and Developmental Disorders, 27(3), 325-332.

Charman, T., Baron-Cohen, S., Swettenham, J., Baird, G., Cox, A., \& Drew, A. (2000). Testing joint attention, imitation, and play as infancy precursors to language and theory of mind. Cognitive Development, 15(4), 481-498.

Chomsky, N. (2000). New horizons in the study of language and mind. Cambridge: Cambridge University Press.

Christmann, U., \& Groeben, N. (2014). Empirische Rezeptionspsychologie der Fiktionalität. In T. Klauk und T. Köppe (Hrsg.), Fiktionalität. Ein interdisziplinäres Handbuch (338-360). Boston (MA) [u. a.]: Walter de Gruyter.

Cook, R., Bird, G., Catmur, C., Press, C., \& Heyes, C. (2014). Mirror neurons: from origin to function. Behavioral and Brain Sciences, 37(02), 177-192.

Corkum, V., \& Moore, C. (1995). Development of joint visual attention in infants. In C. Moore und P. Dunham (Hrsg.), Joint attention: Its origin and role in development. Hillsdale (NJ): Erlbaum.

Currie, G. (1990). The nature of fiction. Cambridge: Cambridge University Press.

Currie, G. (2007). Was ist fiktionale Rede? In M. E. Reicher (Hrsg.), Fiktion, Wahrheit, Wirklichkeit. Philosophische Grundlagen der Literaturtheorie (37-53). Paderborn: Mentis.

Cutting, A. L., \& Dunn, J. (1999). Theory of mind, emotion understanding, language, and family background: Individual differences and interrelations. Child Development, 70(4), 853-865.

Desrochers, S., Morissette, P., \& Ricard, M. (1995). Two perspectives on pointing in infancy. In C. Moore und P. J. Dunham (Hrsg.), Joint attention: Its origins and role in development (85-101). Mahwah: Erlbaum Associates.

de Villiers, P. (2005). The role of language in theory-of-mind development: What deaf children tell us. In J. W. Astington und J. A. Baird (Hrsg.), Why language matters for theory of mind (266-297). Oxford: Oxford University Press.

de Villiers, J. G., \& Pyers, J. E. (2002). Complements to cognition: A longitudinal study of the relationship between complex syntax and false-belief-understanding. Cognitive Development, 17(1), 1037-1060.

Doherty, M., \& Perner, J. (1998). Metalinguistic awareness and theory of mind: Just two words for the same thing? Cognitive Development, 13(3), 279-305. 
Dunbar, R. (2000). On the origin of the human mind. In P. Carruthers und A. Chamberlain (Hrsg.), Evolution and the Human Mind: Modularity, Language and MetaCognition (238-253). Cambridge: Cambridge University Press.

Eagleton, T. (1996). Literary theory: An introduction. Minneapolis (MN): University of Minnesota Press.

Edwards, P., \& Pálsson, H. (2006). The Book of Settlements: Landnámabók. Manitoba: University of Manitoba Press.

Eilan, N., Hoerl, C., McCormack, T., \& Roessler, J. (Hrsg.). (2005). Joint attention: Communication and other minds: Issues in philosophy and psychology. Oxford: Oxford University Press.

Fadiga, L., Craighero, L., Fabbri Destro, M., Finos, L., Cotillon-Williams, N., Smith, A. T., \& Castiello, U. (2006). Language in shadow. Social Neuroscience, 1(2), 77-89.

Flavell, J. H. (1986). The development of children's knowledge about the appearancereality distinction. American Psychologist, 41(4), 418-425.

Flavell, J. H., Flavell, E. R., \& Green, F. L. (1983). Development of the appearance-reality distinction. Cognitive Psychology, 15(1), 95-120.

Flavell, J. H. (1993). The Development of Children's Understanding of False Belief and the Appearance-Reality Distinction. International Journal of Psychology, 28(5), 595604.

Fogassi, L., Ferrari, P. F., Gesierich, B., Rozzi, S., Chersi, F., \& Rizzolatti, G. (2005). Parietal lobe: from action organization to intention understanding. Science, 308, 662-667.

Fox, D. (1963). Njáls Saga and the Western Literary Tradition. Comparative Literature, 15, 289-310.

Friederici, A. D., Bahlmann, J., Heim, S., Schubotz, R. I., \& Anwander, A. (2006). The brain differentiates human and non-human grammars: Functional localization and structural connectivity. Proceedings of the National Academy of Sciences of the United States of America, 103(7), 2458-2463.

Friend, M., \& Davis, T. L. (1993). Appearance-reality distinction: Children's understanding of the physical and affective domains. Developmental Psychology, 29(5), 907-914.

Frye, D., Zelazo, P. D., \& Palfai, T. (1995). Theory of mind and rule-based reasoning. Cognitive Development, 10(4), 483-527.

Gallagher, S., \& Hutto, D. (2008). Understanding others through primary interaction and narrative practice. In J. Zlatev, T. P. Racine, C. Sinha und E. Itkonen (Hrsg.), The shared mind: Perspectives on intersubjectivity (17-38). Amsterdam [u. a.]: John BEnjamins Publishing Company.

Gallese, V. (2006). Intentional attunement: A neurophysiological perspective on social cognition and its disruption in autism. Brain Research, 1079(1), 15-24. 
Gallese, V. (2007). Before and below ,theory of mind': Embodied simulation and the neural correlates of social cognition. Philosophical Transactions of the Royal Society of London B: Biological Sciences, 362, 659-669.

Gallese, V. (2008). Mirror neurons and the neural exploitation hypothesis: From embodied simulation to social cognition. In J. A. Pineda (Hrsg.), Mirror Neuron Systems (163-190). New York: Humana Press.

Gallese, V., \& Goldman, A. (1998). Mirror neurons and the simulation theory of mindreading. Trends in Cognitive Sciences, 2(12), 493-501.

Genette, G. (2010). Die Erzählung (3. Aufl.). München: Wilhelm Fink. (Origninalwerk veröffentlicht 1972).

Gergely, G., \& Csibra, G. (2003). Teleological reasoning in infancy: The naive theory of rational action. Trends in Cognitive Sciences, 7(7), 287-292.

Goldman, A. (2006a). Simulating minds: The philosophy, psychology, and neuroscience of mindreading. Oxford: Oxford University Press.

Goldman, A. (2006b). Imagination and simulation in audience responses to fiction. In S. Nichols (Hrsg.), The Architecture of the Imagination: New Essays on Pretense, Possibility and Fiction, Oxford University Press, New York (41-56). Oxford: Oxford University Press.

Hale, C. M., \& Tager-Flusberg, H. (2003). The influence of language on theory of mind: A training study. Developmental Science, 6(3), 346-359.

Hamlin, J. K., Hallinan, E. V., \& Woodward, A. L. (2008). Do as I do: 7-month-old infants selectively reproduce others' goals. Developmental Science, 11(4), 487-494.

Happe, F. G. (1995). The role of age and verbal ability in the theory of mind task performance of subjects with autism. Child Development, 66(3), 843-855.

Harris, P. L. (1992). From simulation to folk psychology: The case for development. Mind \& Language, 7, 120-144.

Harris, P. L. (2000). The work of the imagination. Cambridge (MA) Blackwell Publishing.

Harris, P. L., Donnelly, K., Guz, G. R., \& Pitt-Watson, R. (1986). Children's understanding of the distinction between real and apparent emotion. Child Development, 57, 895909.

Harris, P. L., Kavanaugh, R. D., Wellman, H. M., \& Hickling, A. K. (1993). Young children's understanding of pretense. Monographs of the Society for Research in Child Development, 58(1, Serial Nr. 231).

Hauk, O., Johnsrude, I., \& Pulvermüller, F. (2004). Somatotopic representation of action words in human motor and premotor cortex. Neuron, 41(2), 301-307.

Hauksson, P., \& Óskarsson, P. (1994). Íslensk stílfrææði. Reykjavík: Mál og Menning.

Hauser, M. D., Chomsky, N., \& Fitch, W. T. (2002). The faculty of language: What is it, who has it, and how did it evolve? Science, 298, 1569-1579. 
Herman, D. (2003). Stories as a tool for thinking. In D. Herman (Hrsg.), Narrative Theory and the Cognitive Sciences (163-192). Stanford (CA): CSLI Publications.

Herman, D. (2013). Storytelling and the sciences of mind. Cambridge (MA): MIT Press.

Hernadi, P. (2002). Why Is Literature: A Coevolutionary Perspective on Imaginative Worldmaking. Poetics Today, 23(1), 21-42.

Hobson, R. P. (2005). What Puts the Jointness into Joint Attention? In N. Eilan, C. Hoerl, T. McCormack und J. Roessler (Hrsg.), Joint attention: Communication and other minds: Issues in philosophy and psychology (185-204). Oxford: Oxford University Press.

Hoogenhout, M., \& Malcolm-Smith, S. (2014). Theory of mind in autism spectrum disorder: Does DSM classification predict development? Research in Autism Spectrum Disorders, 8(6), 597-607.

Huntemann, W. (1990). Artistik und Rollenspiel: Das System Thomas Bernhard. Würzburg: Königshausen \& Neumann.

Hutto, D. (2009). Folk psychology as narrative practice. Journal of Consciousness Studies, 16, 9-39.

Jakobson, R. (1979). Linguistik und Poetik. In E. Holenstein und T. Schelbert (Hrsg.), Poetik. ausgewählte Aufsätze 1921-1971 (83-121). Frankfurt am Main: Suhrkamp.

Jannidis, F. (2004). Figur und Person: Beitrag zu einer historischen Narratologie. Boston (MA) [u. a.]: Walter de Gruyter.

Jaspars, J. M. F., Hewstone, M. R. C., \& Fincham, F. D. (1983). Attribution theory and research: The state of the art. In J. M. F. Jaspars, F. D. Fincham und M. R. C. Hewstone (Hrsg.), Attribution theory and research: Conceptual, developmental and social dimensions (3-36). London: Academic Press.

Jenkins, J. M., \& Astington, J. W. (1996). Cognitive factors and family structure associated with theory of mind development in young children. Developmental Psychology, 32(1), 70-78.

Johnson-Laird, P. N. (1983). Mental models: Towards a cognitive science of language, inference, and consciousness. Cambridge (MA): Harvard University Press.

Johnson-Laird, P. N., Byrne, R. M., \& Schaeken, W. (1992). Propositional reasoning by model. Psychological review, 99(3), 418-439.

Jurgensen, M. (1981). Die Sprachpartituren des Thomas Bernhard. In M. Jurgensen (Hrsg.), Bernhard: Annäherungen (99-122). Bern [u. a.]: Francke.

Kahrs, P. (2000). Thomas Bernhards frühe Erzählungen: rhetorische Lektüren. Würzburg: Königshausen \& Neumann.

Kidd, D. C., \& Castano, E. (2013). Reading literary fiction improves Theory of Mind. Science, 342, 377-380. 
Kintsch, W. (1998). Comprehension: A paradigm for cognition. Cambridge: Cambridge University Press.

Kintsch, W., \& Van Dijk, T. A. (1978). Toward a model of text comprehension and production. Psychological Review, 85(5), 363-394.

Király, I., Jovanovic, B., Prinz, W., Aschersleben, G., \& Gergely, G. (2003). The early origins of goal attribution in infancy. Consciousness and Cognition, 12(4), 752769.

Kochanska, G., Murray, K., Jacques, T. Y., Koenig, A. L., \& Vandegeest, K. A. (1996). Inhibitory control in young children and its role in emerging internalization. Child Development, 67, 490-507.

Kohler, E., Keysers, C., Umilta, M. A., Fogassi, L., Gallese, V., \& Rizzolatti, G. (2002). Hearing sounds, understanding actions: action representation in mirror neurons. Science, 297, 846-848.

Köppe, T. (2014). Die Institution Fiktionalität. In T. Klauk und T. Köppe (Hrsg.), Fiktionalität: Ein interdisziplinäres Handbuch (35-49). Boston (MA) [u. a.]: Walter de Gruyter.

Leekam, S. R. (1991). Jokes and lies: Children's understanding of intentional falsehood. In A. Whiten (Hrsg.), Natural theories of mind: Evolution, development and simulation of everyday mindreading (159-174). Oxford: Blackwell.

Leslie, A. M. (1987). Pretense and representation: The origins of ,theory of mind'. Psychological Review, 94(4), 412-426.

Leslie, A. M. (1992). Pretense, autism, and the theory-of-mind module. Current Directions in Psychological Science, 1(1), 18-21.

Leslie, A. M. (2000). Theory of mind as a mechanism of selective attention. In M. Gazzaniga (Hrsg.), The new Cognitive Neurosciences (1235-1247). Cambridge (MA): MIT Press.

Liddle, B., \& Nettle, D. (2006). Higher-order theory of mind and social competence in school-age children. Journal of Cultural and Evolutionary Psychology, 4(3-4), 231244.

Lillard, A. S. (1993). Pretend play skills and the child's theory of mind. Child Development, 64(2), 348-371.

Liestøl, K. (1930). The origin of the Icelandic family sagas. Oslo: Aschehoug.

Lockl, K., Schwarz, S., \& Schneider, W. (2004). Sprache und Theory of Mind. Zeitschrift für Entwicklungspsychologie und Pädagogische Psychologie, 36(4), 207-220.

Long, J. J. (2001). The novels of Thomas Bernhard: form and its function. Columbia (SC): Camden House.

Lönnroth, L. (1976). Njáls saga: a critical introduction. Berkely (CA): University of California Press. 
Lough, S., Kipps, C. M., Treise, C., Watson, P., Blair, J. R., \& Hodges, J. R. (2006). Social reasoning, emotion and empathy in frontotemporal dementia. Neuropsychologia, 44(6), 950-958.

Luther, S. (2016a). Kognitive Experimente. Über den Zusammenhang zwischen , Theory of Mind' und ,Motivierung' in literarischen Erzähltexten. In M. Horváth und K. Mellmann (Hrsg.), Die biologisch-kognitiven Grundlagen narrativer Motivierung (55-74). Paderborn: Mentis.

Luther, S. (2016b). Wahrnehmungskontexte und ihre ,Mentale' Verknüpfung. Welche Rolle spielt ,Joint Attention' für das Verstehen literarischer Erzähltexte? In R. Mikuláš und S. Wege (Hrsg.), Schlüsselkonzepte und Anwendungen der Kognitiven Literaturwissenschaft (165-184). Paderborn: Mentis.

Magliano, J. P., Dijkstra, K., \& Zwaan, R. A. (1996). Generating predictive inferences while viewing a movie. Discourse Processes, 22(3), 199-224.

Mansour, J. (2009). Chancen und Grenzen des Transfers kognitionspsychologischer Annahmen und Konzepte in der Literaturwissenschaft - das Beispiel der Theory of Mind. In M. Huber (Hrsg.), Literatur und Kognition. Bestandsaufnahmen und Perspektiven eines Arbeitsfeldes (155-163). Paderborn: Mentis.

Mar, R. A. (2011). The neural bases of social cognition and story comprehension. Annual Review of Psychology, 62, 103-134.

Martinez, M., \& Scheffel, M. (2007). Einführung in die Erzähltheorie (7. Aufl.). München: C. H. Beck.

Meltzoff, A. N. (1995). Understanding the intentions of others: Re-enactment of intended acts by 18-month-old children. Developmental Psychology, 31(5), 838.

Miall, D. S., \& Kuiken, D. (1994). Foregrounding, defamiliarization, and affect: Response to literary stories. Poetics, 22(5), 389-407.

Miall, D. S., \& Kuiken, D. (1998). The form of reading: Empirical studies of literariness. Poetics, 25(6), 327-341.

Miall, D. S., \& Kuiken, D. (1999). What is literariness? Three components of literary reading. Discourse Processes, 28(2), 121-138.

Miall, D. S. (2006). Empirical approaches to studying literary readers: The state of the discipline. Book History, 9(1), 291-311.

Moll, H., \& Meltzoff, A. N. (2011). Foundation in Joint Attention. In J. Roessler, H. Lerman und N. Eilan (Hrsg.), Perception, causation, and objectivity (286-304). Oxford: Oxford University Press.

Mukařowský, Jan: Standard language and poetic language. In L. Burge, T. Crowly und A. Girvin (Hrsg.), The Routledge Language and Cultural Theory Reader (225-230). London [u. a.]: Routledge.

Newcombe, N., \& Huttenlocher, J. (1992). Children's early ability to solve perspectivetaking problems. Developmental Psychology, 28(4), 635-643. 
Nichols, S. (2004). Imagining and believing: The promise of a single code. The Journal of Aesthetics and Art Criticism, 62(2), 129-139.

Nichols, S., \& Stich, S. P. (2003). Mindreading: An integrated account of pretence, selfawareness, and understanding other minds. New York: Clarendon Press.

Nünning, A. (2001). On the perspective structure of narrative texts: Steps toward a constructivist narratology. In W. v. Peer und S. Chatman (Hrsg.), New perspectives on narrative perspective (207-223). New York: State University of New York Press.

Palmer, A. (2004). Fictional Minds. Lincoln (NE): University of Nebraska Press.

Perner, J. (1991). Understanding the representational mind. Cambridge (MA): The MIT Press.

Perner, J., Brandl, J. L., \& Garnham, A. (2003). What is a perspective problem? Developmental issues in belief ascription and dual identity. Facta Philosophica, 5, 355-378.

Perner, J., Stummer, S., Sprung, M., \& Doherty, M. (2002). Theory of mind finds its Piagetian perspective: Why alternative naming comes with understanding belief. Cognitive Development, 17(3), 1451-1472.

Perner, J., Frith, U., Leslie, A. M., \& Leekam, S. R. (1989). Exploration of the autistic child's theory of mind: Knowledge, belief, and communication. Child Development, $60,689-700$.

Premack, D., \& Woodruff, G. (1978). Does the chimpanzee have a theory of mind? Behavioral and Brain Sciences, 1(04), 515-526.

Prince, Gerald (1985). The narratee revisited. Style, 19(3), 299-303.

Rakoczy, H. (2004). The development of performing and understanding pretend play: a cultural learning perspective (Dissertation). Universität Leipzig, Leipzig.

Abgerufen von: https://www.psych.uni-

goettingen.de/de/development/team/rakoczy-hannes/publikationen-1/submitted/thedevelopment-of-performing-and-understanding-pretend-play-a-cultural-learningperspective. Zuletzt eingesehen am 26.06.2017.

Rakoczy, H. (2008). Taking fiction seriously: young children understand the normative structure of joint pretence games. Developmental Psychology, 44(4), 1195-1201.

Repacholi, B. M., \& Gopnik, A. (1997). Early reasoning about desires: evidence from 14and 18-month-olds. Developmental Psychology, 33(1), 12-21.

Richardson, A. (2010). Facial expression theory from Romanticism to the present. In L. Zunshine (Hrsg.), Introduction to cognitive cultural studies (65-83). Baltimore (MD): The Johns Hopkins University Press.

Rizzolatti, G., Fadiga, L., Gallese, V., \& Fogassi, L. (1996). Premotor cortex and the recognition of motor actions. Cognitive Brain Research, 3(2), 131-141. 
Rizzolatti, G., \& Craighero, L. (2004). The mirror-neuron system. Annual Review of Neuroscience, 27, 169-192.

Sabbagh, M. A., \& Baldwin, D. (2005). Understanding the role of communicative intentions in word learning. In N. Eilan, C. Hoerl, T. McCormack und J. Roessler (Hrsg.), Joint attention: Communication and other minds: Issues in philosophy and psychology (165-184). Oxford: Oxford University Press.

Sandson, J., \& Albert, M. L. (1984). Varieties of perseveration. Neuropsychologia, 22(6), 715-732.

Sarter, M., Givens, B., \& Bruno, J. P. (2001). The cognitive neuroscience of sustained attention: where top-down meets bottom-up. Brain Research Reviews, 35(2), 146160.

Saxe, R., Schulz, L. E., \& Jiang, Y. V. (2006). Reading minds versus following rules: Dissociating theory of mind and executive control in the brain. Social Neuroscience, 1, 284-298.

Schroeder, T., \& Matheson, C. (2006). Imagination and emotion. In S. Nichols (Hrsg.), The Architecture of the Imagination: New Essays on Pretense, Possibility and Fiction, Oxford University Press, New York (19-39). Oxford: Oxford University Press.

Searle, J. R. (1982). Ausdruck und Bedeutung. Untersuchungen zur Sprechakttheorie. Frankfurt am Main: Suhrkamp.

Searle, J. R. (2007). Der logische Status fiktionaler Rede. In M. E. Reicher (Hrsg.), Fiktion, Wahrheit, Wirklichkeit. Philosophische Grundlagen der Literaturtheorie (21-36). Paderborn: Mentis.

Shapiro, L. (2010). Embodied cognition. Abingdon: Routledge.

Shklovsky, V., (1965). Art as Technique. In L. T. Lemon und M. Reis (Hrsg.), Russian formalist criticism: Four essays (3-24). Lincoln (NE): University of Nebraska Press.

Stockwell, P. (2005). Cognitive poetics: An introduction. Abongdon: Routledge.

Sveinsson, E. Ó., \& Schach, P. (1971). Njáls saga: A literary masterpiece. Lincoln (NE): University of Nebraska Press.

Tager-Flusberg, H., \& Joseph, R. M. (2005). How language facilitates the acquisition of false-belief understanding in children with autism. In J. W. Astington und J. A. Baird (Hrsg.), Why language matters for theory of mind (298-318). Oxford: Oxford University Press.

Taylor, H. A., \& Tversky, B. (1992). Spatial mental models derived from survey and route descriptions. Journal of Memory and Language, 31(2), 261-292.

Tomasello, M. (1995). Joint attention as social cognition. In C. Moore und P. J. Dunham (Hrsg.), Joint attention: Its origins and role in development (103-130). Mahwah: Erlbaum Associates. 
Umiltà, M. A., Kohler, E., Gallese, V., Fogassi, L., Fadiga, L., Keysers, C., \& Rizzolatti, G. (2001). I know what you are doing: A neurophysiological study. Neuron, 31(1), 155-165.

Walton, K. L. (1990). Mimesis as make-believe: On the foundations of the representational arts. Boston: Harvard University Press.

Walton, K. (1997). Spelunking, simulation, and slime: On being moved by fiction. In M. Hjort und S. Laver (Hrsg.), Emotion and the Arts (37-49). Oxford: Oxford University Press.

Walton, K. L. (2007). Furcht vor Fiktionen. In M. E. Reicher (Hrsg.), Fiktion, Wahrheit, Wirklichkeit. Philosophische Grundlagen der Literaturtheorie (94-119). Paderborn: Mentis.

Wege, S. (2013). Wahrnehmung-Wiederholung-Vertikalität: zur Theorie und Praxis der Kognitiven Literaturwissenschaft. Bielefeld: Aisthesis-Verlag.

Wellman, H. M., Cross, D., \& Watson, J. (2001). Meta-analysis of theory-of-mind development: the truth about false belief. Child Development, 72(3), 655-684.

Wellman, H. M., \& Liu, D. (2004). Scaling of theory-of-mind tasks. Child Development, 75(2), 523-541.

Wicker, B., Keysers, C., Plailly, J., Royet, J. P., Gallese, V., \& Rizzolatti, G. (2003). Both of us disgusted in my insula: The common neural basis of seeing and feeling disgust. Neuron, 40(3), 655-664.

Wimmer, H., \& Perner, J. (1983). Beliefs about beliefs: Representation and constraining function of wrong beliefs in young children's understanding of deception. Cognition, 13(1), 103-128.

Winko, S. (2009). Auf der Suche nach der Weltformel. Literarizität und Poetizität in der neueren literaturtheoretischen Diskussion. In S. Winko, F. Jannidis und G. Lauer (Hrsg.), Grenzen der Literatur. Zu Begriff und Phänomen des Literarischen 374396). Boston (MA) [u. a.]: Walter de Gruyter.

Winner, E., Brownell, H., Happé, F., Blum, A., \& Pincus, D. (1998). Distinguishing lies from jokes: Theory of mind deficits and discourse interpretation in right hemisphere brain-damaged patients. Brain and Language, 62(1), 89-106.

Woodward, A. L. (1998). Infants selectively encode the goal object of an actor's reach. Cognition, 69(1), 1-34.

Wyer Jr, R. S. (2007). Principles of mental representation. In A. W. Kruglanski und E. T. Higgins (Hrsg.), Social psychology: Handbook of basic principles (285-307). New York: The Guilford Press.

Youngblade, L. M., \& Dunn, J. (1995). Individual differences in young children's pretend play with mother and sibling: Links to relationships and understanding of other people's feelings and beliefs. Child Development, 66(5), 1472-1492. 
Zipfel, F. (2001). Fiktion, Fiktivität, Fiktionalität: Analysen zur Fiktion in der Literatur und zum Fiktionsbegriff in der Literaturwissenschaft. Berlin: Erich Schmidt Verlag.

Zubin, D. A., \& Hewitt, L. E. (1995). The deictic center: A theory of deixis in narrative. In J. F. Duchan, G. A. Bruder und L. E. Hewitt (Hrsg.), Deixis in narrative: A cognitive science perspective (129-155). Mahwah (NJ): Erlbaum Associates.

Zunshine, L. (2006). Why we read fiction: theory of mind and the novel. Columbus: Ohio State University Press.

Zunshine, L. (2010). Lying bodies of the Enlightenment: Theory of mind and cultural historicism. In L. Zunshine (Hrsg.), Introduction to cognitive cultural studies (115133). Baltimore (MD): The Johns Hopkins University Press.

Zwaan, R. A. (1999). Five dimensions of narrative comprehension: The event-indexing model. In S. R. Goldman, A. C. Graesser und P. v. d. Broek (Hrsg.), Narrative comprehension, causality, and coherence: Essays in honor of Tom Trabasso (93110). Mahwah (NJ): Erlbaum Associates.

Zwaan, R. A., Langston, M. C., \& Graesser, A. C. (1995). The construction of situation models in narrative comprehension: An event-indexing model. Psychological Science, 6(5), 292-297.

Zwaan, R. A., \& Madden, C. J. (2004). Updating situation models. Journal of Experimental Psychology: Learning, Memory, and Cognition, 30, 283-288.

Zwaan, R. A., \& Radvansky, G. A. (1998). Situation models in language comprehension and memory. Psychological Bulletin, 123(2), 162-185.

\section{Primärliteratur:}

Bernhard, T. (2013). Gehen. Frankfurt am Main: Suhrkamp.

Melville, H. (1994). Moby-Dick. London: Penguin Books.

Nabokov, V. V. (1960). Laughter in the Dark. New York (NY): New Directions Publishing.

Njál's Saga. (1998). Ware, Hertfordshire: Wordsworth Editions.

Sveinsson, E. Ó. (Hrsg.). (1954). Brennu-Njáls saga. Reykjavík: Hið Íslenzka Fornritafélag.

Woolf, V. (1960). Mrs. Dalloway. London: Hogarth Press. 


\section{Zeitungsartikel}

Mass Hysteria in U.S.A. Radio Broadcast Panic. The Age, 2. November 1938, abgerufen von https://news.google.com/newspapers?id=bJ1VAAAAIBAJ\&sjid= JJcDAAAAIBAJ\&pg=2609\%2C186374, zuletzt eingesehen am 12.05.2017. 


\section{Eidesstattliche Erklärung}

\section{Versicherung gemäß $§ 9$ der Promotionsordnung (gültig seit 1.10.2015) der Philosophischen Fakultät der Universität Göttingen.}

Hiermit versichere ich, dass ich die vorliegende Dissertation selbstständig angefertigt habe und dass ich keine anderen als die angegebenen Quellen und Hilfsmittel benutzt habe. Des Weiteren versichere ich, dass anderweitig keine entsprechende Promotion beantragt wurde und hierbei die eingereichte Dissertation oder Teile daraus vorgelegt worden sind. 